\title{
Investigation of Mitochondrial Transfer between Human Erythroblasts
}

\author{
Brittany Lewer
}

A thesis submitted to Victoria University of Wellington in fulfilment of the requirements for the degree of Master of Biomedical Science 


\section{Acknowledgements}

Firstly, I have to acknowledge my supervisors; Dr Melanie McConnell, Dr Robert Weinkove and Professor Mike Berridge.

To Melanie, thank you for offering me this project. I have learnt so many skills and had so many opportunities throughout these last 2 years because of you. Your patience, unwavering support, guidance and honesty helped me grow as a scientist!

To Rob, thank you for providing an optimism that rivalled my own. You provided me invaluable insight into the clinical-research interface of this project. Your support and clinical knowledge continually helped to shape my work in this project.

To Mike, thank you for being an endless wealth of knowledge I only just scratched the surface of.

I want to thank everyone in the Berridge and McConnell labs for your continued support and encouragement. A special thanks to Matt and Georgia for putting up with my continuous questions, optimism and philosophical scientific chats. I couldn't have made it through this project without your help!

Thank you to everyone at the Malaghan Institute for so many things. Thanks to my past and present Siberia office friends for endless encouragement, support and well needed distractions. My most important experiments could not have been done without the HGCC, Sally, Sam, and Alfonso. I want to thank you for all your help, the life advice and chat in the sort room. To all the support and technical staff, none of this research could be done without you.

I could not have done this without my amazing friends. Thank you for your endless encouragement and support and cheering me up on bad days.

To Thomas, words cannot express how grateful I am to have had you by my side every day of this project. You went above and beyond to support me in this endeavour.

Finally, thank you to my whole family, especially Mum, Dad and Teagan. You set me up for success and helped me believe I could do anything. Thank you for loving and supporting me every step of the way unconditionally. 


\section{Abstract}

The increasingly studied phenomenon of mitochondria transferring between cells contrasts the popular belief that mitochondria reside permanently within their cells of origin. Research has identified this process occurring in many tissues such as brain, lung and more recently within the bone marrow. This project aimed to investigate if mitochondria could be transferred between human erythroblasts, a context not previously studied.

Tissue microenvironments can be modelled using co-culture systems. Fluorescence activated cell sorting and a highly sensitive Allele-Specific-Blocker qPCR assay were used to leverage mitochondrial DNA polymorphisms between co-cultured populations. Firstly, HL-60 po bone marrow cells, without mitochondrial DNA, deprived of essential nutrients pyruvate and uridine were co-cultured in vitro with HEL cells, a human erythroleukemia. Secondly, HEL cells treated with deferoxamine or cisplatin, were cocultured with parental HL-60 cells in vitro. Lastly, ex vivo co-cultures between erythroblasts differentiated from mononuclear cells in peripheral blood were conducted, where one population was treated with deferoxamine.

Co-culture was able to improve recovery when HL-60 0 o cells were deprived of pyruvate and uridine. Improved recovery was similarly detected for HEL cells treated with deferoxamine after co-culture with HL-60 cells. Transfer of mitochondrial DNA did not occur at a detectable level in any co-culture condition tested. The high sensitivity of the allele-specific-blocker qPCR assay required completely pure populations to analyse, however this was not achieved using FACS techniques. In conclusion, results have not demonstrated but cannot exclude the possibility that erythroid cells transfer mitochondria to each other. 


\section{Table of Contents}

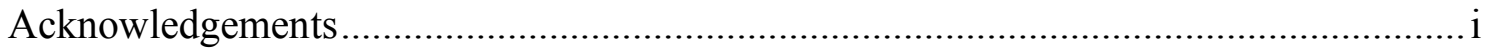

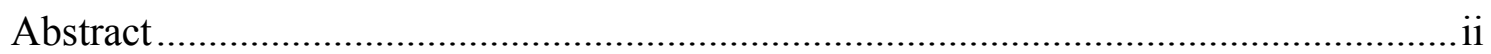

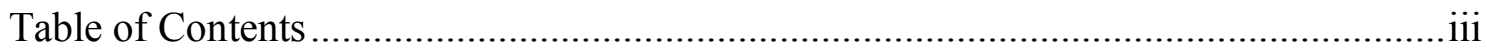

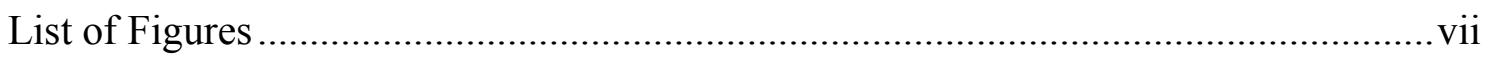

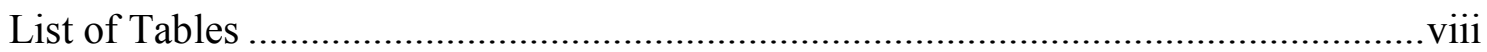

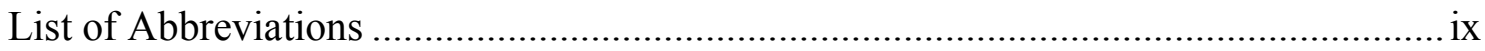

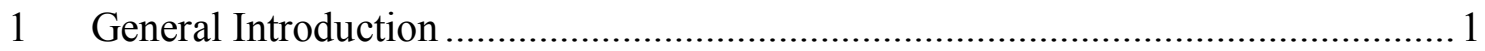

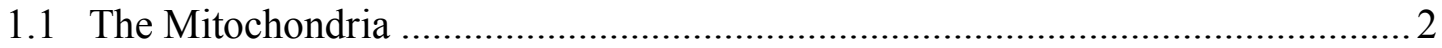

1.1.1 Mitochondrial Structure and Function ................................................... 2

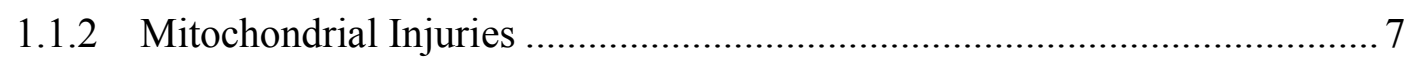

1.2 Mitochondrial Transfer Phenomenon .............................................................. 10

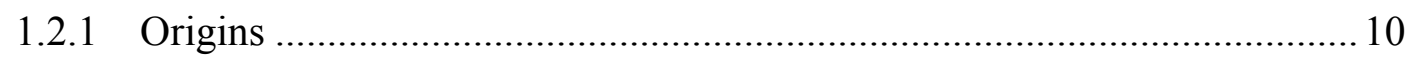

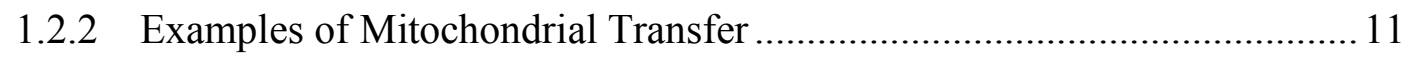

1.2.3 Techniques to identify transfer of mitochondria........................................ 14

1.3 Mitochondrial Transfer in Human Bone Marrow ................................................. 17

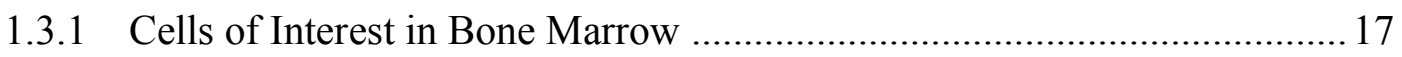

1.3.2 Models of Mitochondrial Transfer between Human Erythroblasts ............20

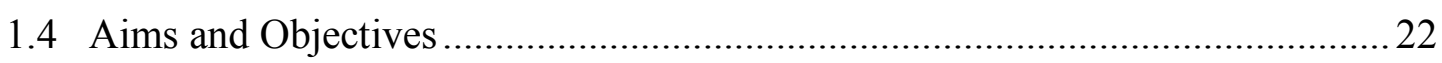

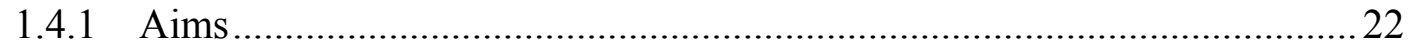

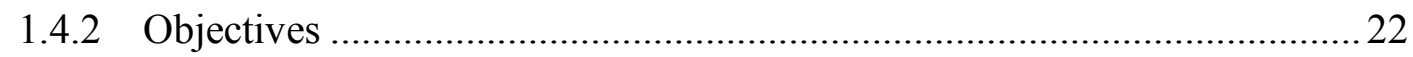

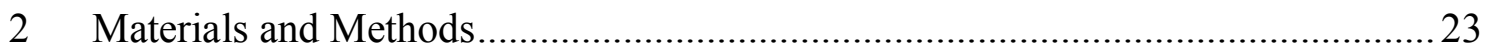

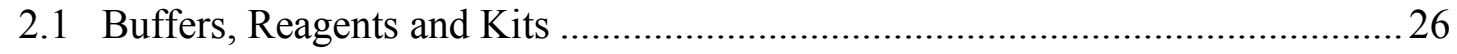

2.1.1 Complete Cell Line Culturing Media ......................................................28

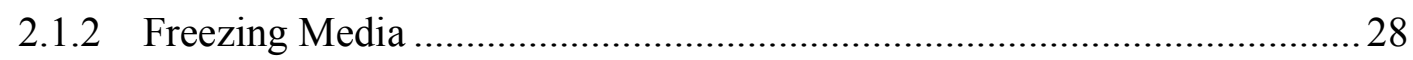

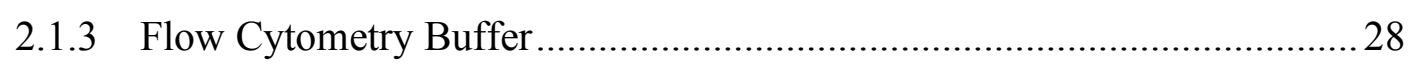

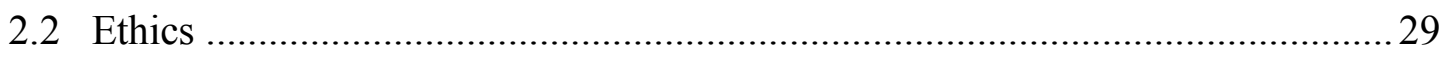

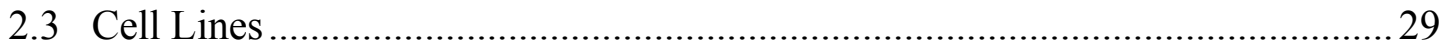

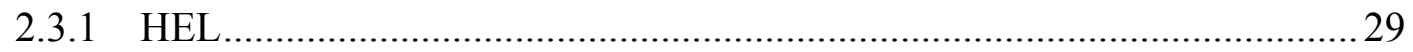

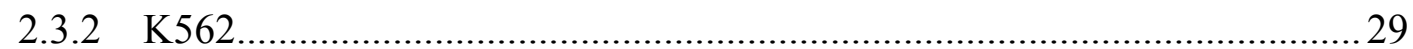




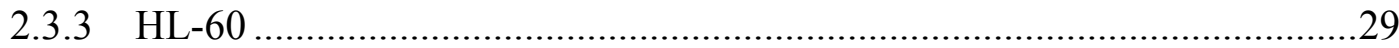

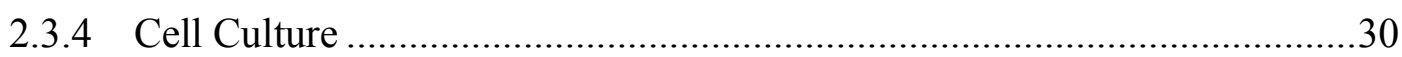

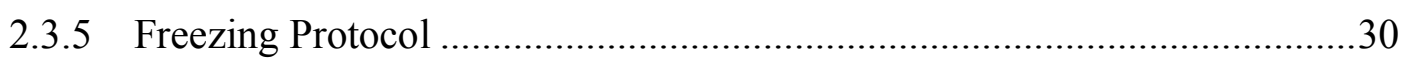

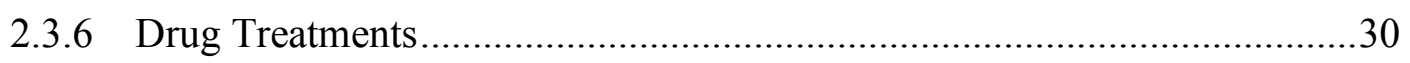

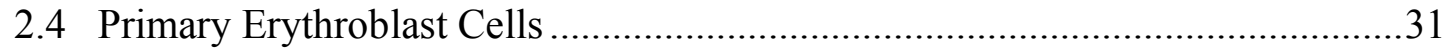

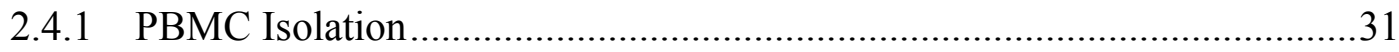

2.4.2 Erythroblast Base Culture Media ...............................................................31

2.4.3 Erythroblast Culture Method....................................................................... 31

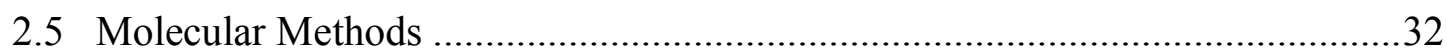

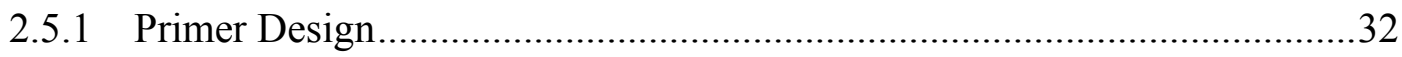

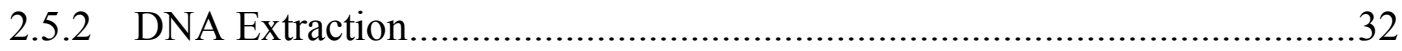

2.5.3 Human Mitochondrial DNA Sequencing .................................................33

2.5.4 Mitochondrial Copy Number Endpoint PCR ….........................................36

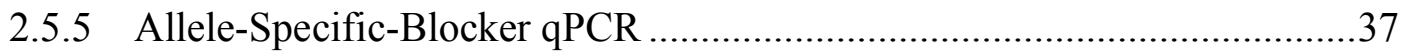

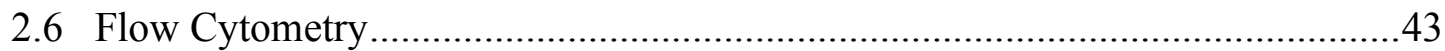

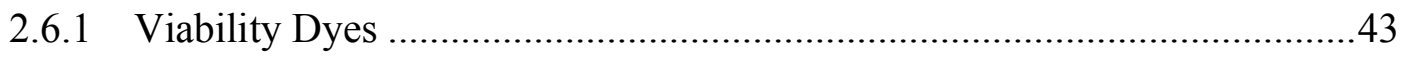

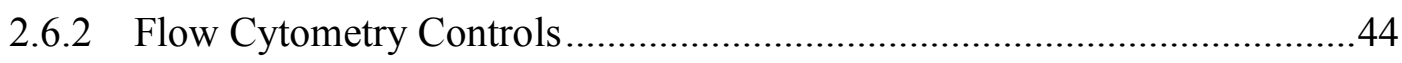

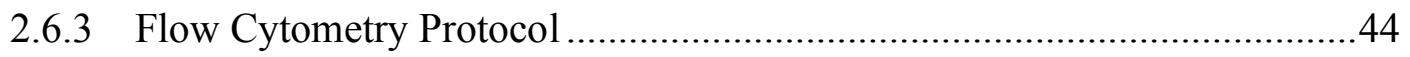

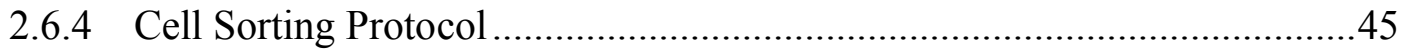

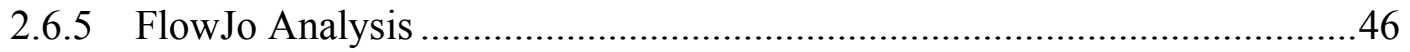

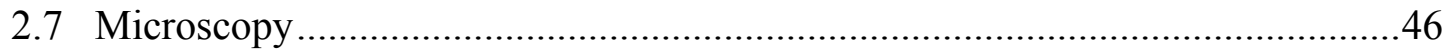

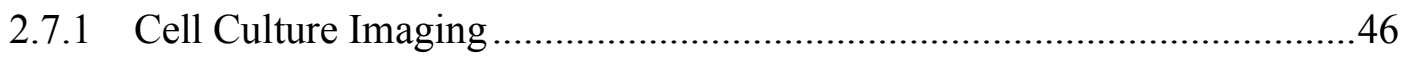

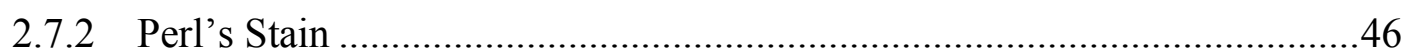

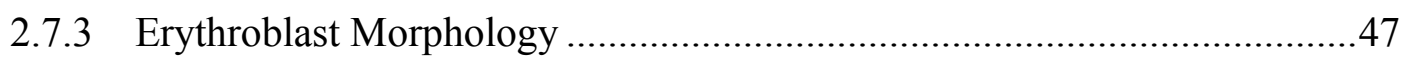

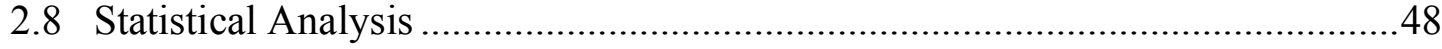

3 Exploration of mitochondrial transfer in cells lacking mitochondrial DNA ...........49

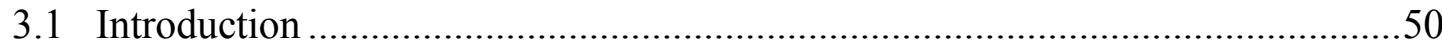

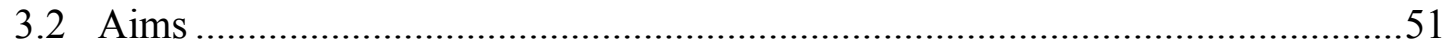

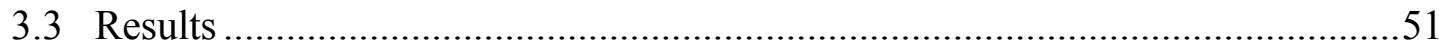

3.3.1 HL-60 po cells lacked detectable mitochondrial DNA and had reduced viability and proliferative capacity when deprived of uridine and pyruvate...........52 3.3.2 HL-60 $\rho$ o cells are protected from declining viability when co-cultured with HEL cells or in HEL conditioned media.............................................................54 
3.3.3 HL-60 po cells do not recover proliferation capacity when co-cultured with HEL cells or in HEL conditioned media.

3.3.4 HL-60 po cells do not uptake detectable levels of HEL mitochondrial DNA from HEL conditioned media. .58

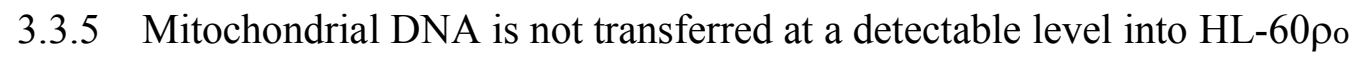
cells when physically co-cultured with HEL cells

3.4 Discussion

3.5 Conclusion 66

4 Investigation of mitochondrial transfer between human bone marrow cell lines in vitro 67

4.1 Introduction 68

4.2 Aims..... 69

4.3 Results .70

4.3.1 HEL cells contained low levels of iron and HL-60 cells expressed CD38.70

4.3.2 Two single nucleotide polymorphisms were leveraged to design an AlleleSpecific-Blocker qPCR assay to detect HL-60 mtDNA transfer. 72

4.3.3 K562 were resistant to Deferoxamine reduction in viability and mitochondrial membrane potential 74

4.3.4 HEL cells were sensitive to deferoxamine reduction in viability and mitochondrial membrane potential .78

4.3.5 HEL cells did not completely recover viability or mitochondrial membrane potential without co-culture after DFO treatment .82

4.3.6 Co-culture with HL-60 restored HEL viability after 24-hour deferoxamine treatment 84

4.3.7 Mitochondrial DNA transfer did not occur to a detectable level between HL-60 and DFO treated HEL cells in co-culture.

4.3.8 Cisplatin treatment induced morphological changes in K562 and reduced viability alongside mitochondrial membrane potential.

4.3.9 HEL cells were highly sensitive to cisplatin treatment reduction in viability and mitochondrial membrane potential .96

4.3.10 HEL cells were unable to recover viability and mitochondrial membrane potential from 24-hour $1 \mu \mathrm{M}$ Cisplatin treatment without co-culture 100 
4.3.11 Co-culture with HL-60 did not rescue cisplatin induced reduction in HEL viability 102

4.3.12 Transfer of HL-60 mitochondrial DNA did not occur to a detectable

level into $1 \mu \mathrm{M}$ Cisplatin treated HEL cells........................................................104

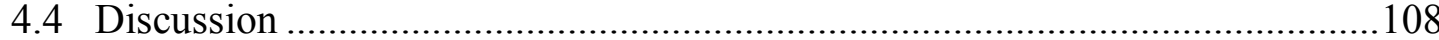

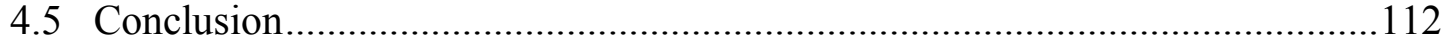

5 Investigation of mitochondrial transfer between primary human erythroblasts $e x$ vivo 113

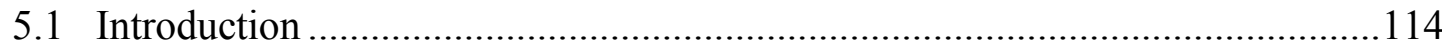

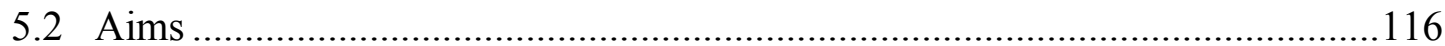

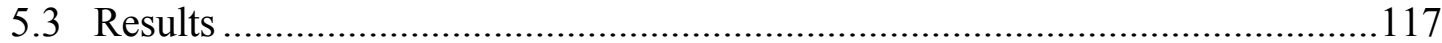

5.3.1 Mixed-culture cells differentiated from PBMCs morphologically resembled erythroblasts in human bone marrow ….............................................................117

5.3.2 Mixed-culture cells expressed common erythroblast surface markers detected by flow cytometry

5.3.3 A mitochondrial DNA polymorphism suitable for detecting transfer in the primary cell model was identified

5.3.4 Deferoxamine increased erythroblast culture apoptosis after 24-hour treatment.

5.3.5 Erythroblast cultures did not completely recover 48-hours after 24-hour deferoxamine treatment

5.3.6 Co-culturing Deferoxamine-treated erythroblasts with untreated erythroblasts did not increase viability above controls

5.3.7 Deferoxamine-treated erythroblasts did not receive detectable mitochondrial DNA from untreated erythroblasts in co-culture

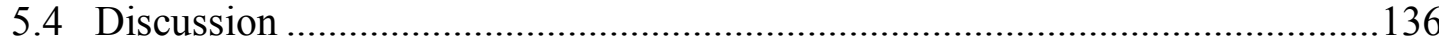

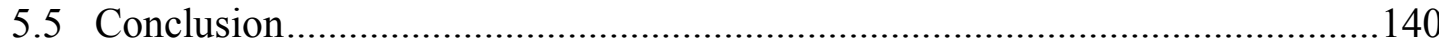

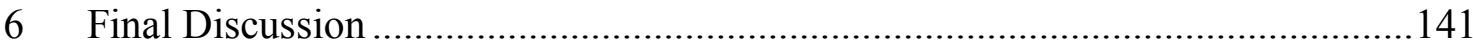

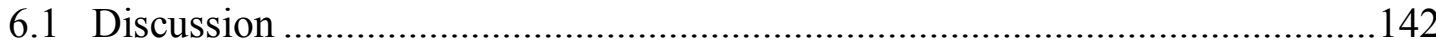

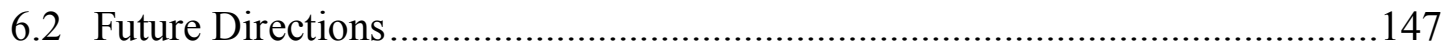

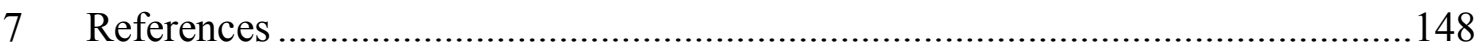




\section{List of Figures}

Figure 1.1: Complexes of the Electron Transport Chain. …........................................... 5

Figure 1.2: Electron Transport Chain complexes in $\rho 0$ cells. ....................................... 9

Figure 1.3: Postulated mechanisms of Mitochondrial Transfer................................... 12

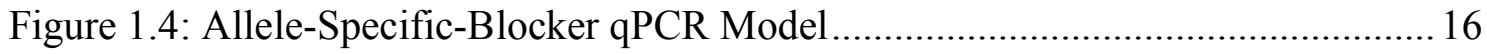

Figure 1.5: Hematopoietic, Mesenchymal and Erythroid Differentiation. ..................... 19

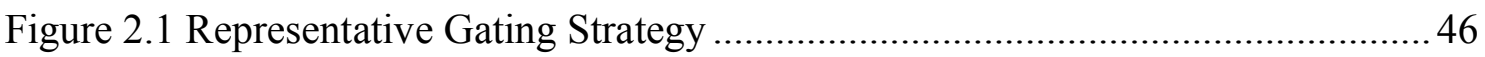

Figure 3.1: HL-60 0 o cells lacked detectable mitochondrial DNA and had reduced viability and proliferative capacity when deprived of uridine and pyruvate. .........53

Figure 3.2: HL-60 $\rho 0$ cells are protected from declining viability when co-cultured with HEL cells but not in HEL conditioned media.

Figure 3.3: HL-60 $\rho 0$ cells do not recover proliferation capacity when co-cultured with

HEL cells or in HEL conditioned media.

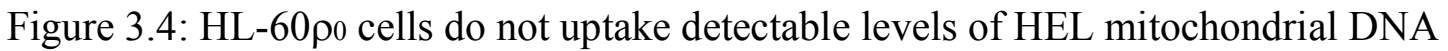
from HEL conditioned media.

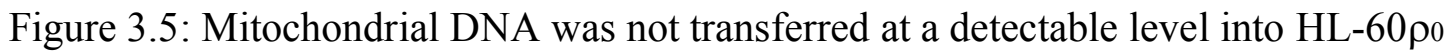
cells when physically co-cultured with HEL cells 62

Figure 4.1: HEL cells contained low levels of iron and HL-60 cells expressed CD38. . 71

Figure 4.2: Two single nucleotide polymorphisms were leveraged to design an Allele-

Specific-Blocker qPCR assay to detect HL-60 mtDNA transfer.

Figure 4.3: K562 were somewhat resistant to Deferoxamine reduction in viability and

mitochondrial membrane potential.

Figure 4.4: HEL cells were sensitive to deferoxamine reduction in viability and mitochondrial membrane potential. 80

Figure 4.5: HEL cells did not completely recover viability or mitochondrial membrane potential without co-culture after 24 -hour $10 \mu \mathrm{M}$ or $25 \mu \mathrm{M}$ Deferoxamine treatment.

Figure 4.6: Co-culture with HL-60 restored HEL viability after 24-hour deferoxamine treatment. .86

Figure 4.7: Mitochondrial DNA transfer did not occur to a detectable level between HL60 and deferoxamine treated HEL cells in co-culture. .89

Figure 4.8: Cisplatin treatment induced morphological changes in K562 and reduced viability alongside mitochondrial membrane potential. 
viii

Figure 4.9; HEL cells were highly sensitive to cisplatin treatment reduction in viability and mitochondrial membrane potential.

Figure 4.10: HEL cells were unable to recover viability and mitochondrial membrane potential from 24-hour $1 \mu \mathrm{M}$ Cisplatin treatment without co-culture. 101

Figure 4.11: Co-culture with HL-60 did not rescue cisplatin induced reduction in HEL viability.

Figure 4.12: Transfer of HL-60 mitochondrial DNA did not occur to a detectable level into $1 \mu \mathrm{M}$ Cisplatin treated HEL cells.

Figure 5.1: Mixed-culture cells from PBMCs morphologically resembled erythroblasts in human bone marrow.

Figure 5.2: Mixed culture cells expressed common erythroblast surface markers detected by flow cytometry.

Figure 5.3: A mitochondrial DNA polymorphism suitable for detecting transfer in the primary cell model was identified.

Figure 5.4: Deferoxamine increased erythroblast culture apoptosis after a 24-hour treatment.

Figure 5.5: Erythroblast cultures did not fully recover 48 hours after a 24-hour Deferoxamine treatment.

Figure 5.6: Co-culture after Deferoxamine treatment did not increase viability above controls.

Figure 5.7: Deferoxamine-treated erythroblasts did not receive detectable mitochondrial DNA from untreated erythroblasts in co-culture. 135

\section{List of Tables}

Table 2.1: List of Laboratory Consumables .............................................................24

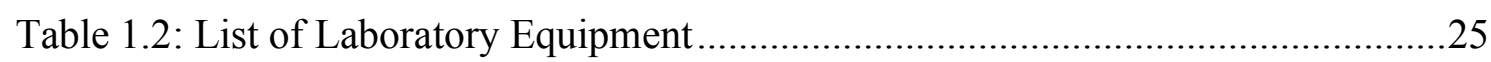

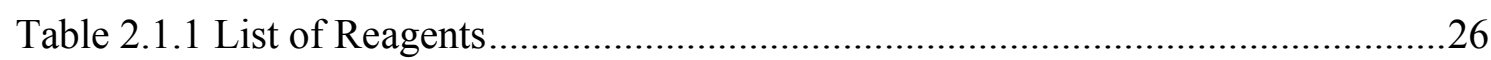

Table 2.5.1 List of Primers for PCR and Sequencing ....................................................33

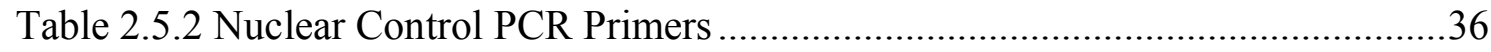

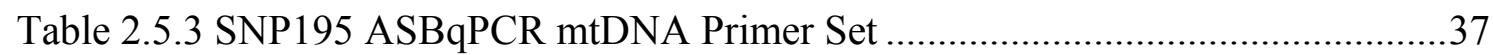

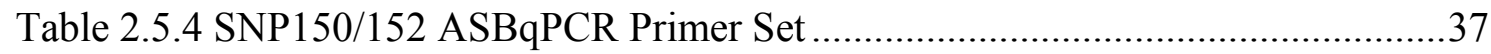

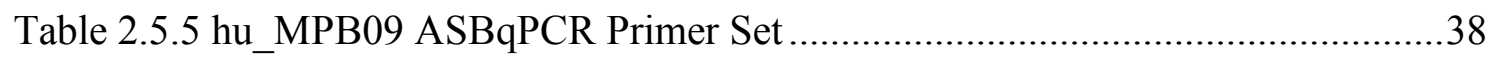


Table 2.6.1 List of Cell Surface Antibodies .43

Table 2.6.2 List of Fluorescent Dyes and Proteins . .43

\section{List of Abbreviations}

$\begin{array}{ll}\text { Acetyl-CoA } & \text { Acetyl coenzyme A } \\ \text { ADP } & \text { Adenosine diphosphate } \\ \text { AML } & \text { Acute myeloid leukemia } \\ \text { ASBqPCR } & \text { Allele-specific-blocker quantitative polymerase chain reaction } \\ \text { ATP } & \text { Adenosine triphosphate }\end{array}$

BMSC

Bone marrow stromal cells

CD

Cluster of differentiation

CLP

Common lymphoid progenitor

CMP

Common myeloid progenitor

$\mathrm{CO} 2$

Carbon dioxide

CoQ

Coenzyme Q

$\mathrm{Ct}$

Cycle threshold

cyt c

Cytochrome c

DAPI

4',6-diamidino-2-phenylindole

DFO

Deferoxamine 
DHODH Dihydroorotate dehydrogenase

D-loop Displacement loop

dMEM Dulbecco's modified eagle medium

DMSO Dimethyl sulfoxide

DNA Deoxyribonucleic acid

dPBS Dulbecco's Phosphate buffer saline

EDTA Ethylenediaminetetraacetic acid

EPO Erythropoietin

ETC Electron transport chain

FACS Fluorescence associated cell sorting

FAD Flavin adenine dinucleotide

FBS Fetal bovine serum

FISH Fluorescent in-situ hybridisation

FMO Fluorescence minus one

FSC Forward scatter

gDNA genomic DNA

HCL Hydrochloric acid

HEPES 4-(2-hydroxyethyl)-1-piperazineethanesulfonic acid 
hMSC

HSC

IL

IMDM

MIMR

MM

MSC

mtDNA

mUNG

$\mathrm{NaCl}$

NAD

NADP

NIR

NSG

NTC

NUMT

คo

PBMC
Human mesenchymal stem cells

Hematopoietic stem cell

Inter-leukin

Iscove's Modified Dulbecco's Medium

Malaghan Institute of Medical Research

Multiple myeloma

Mesenchymal stem cell

mitochondrial DNA

Mitochondrial uracil-N-glycosylase

Sodium chloride

Nicotinamide adenine dinucleotide

Nicotinamide adenine dinucleotide phosphate

Near Infrared

NOD scid gamma

No template control

Nuclear sequences of mitochondrial origin

rho zero

Peripheral blood mononuclear cells 
PCR Polymerase chain reaction

PI

Propidium iodide

qPCR

Quantitative polymerase chain reaction

$\mathrm{RBC}$

Red blood cell

RCA

Rolling circle amplification

RNA

Ribonucleic acid

ROS

Reactive oxygen species

RPMI

Roswell Park Memorial Institute

SCF

Stem cell factor

SNP

Single nucleotide polymorphism

SSC

Side scatter

STR

Short tandem repeat

TCA

Tri-carboxylic acid

tRNA

transfer RNA 
1 General Introduction 


\subsection{The Mitochondria}

\subsubsection{Mitochondrial Structure and Function}

Mitochondria are an integral organelle for eukaryotic cell function. They produce the largest contribution to the energy pool of cells in the form on adenosine tri-phosphate (ATP), and produce many molecules that are shuttled into other pathways for cells function 1. Additionally, mitochondria release cytochrome $\mathrm{c}$ into the cytoplasm in response to stimuli that propagate cellular apoptosis 2,3 .

Individual mitochondria have a unique structure formed by an outer membrane and an inner membrane with an intermembrane space 4 . The outer membrane provides the basic shape of the mitochondria while the inner membrane has convolutions called cristae. The proteins involved in the mitochondrial electron transport chain span this inner membrane. The space inside the inner membrane, called the matrix, contains many ribosomes, proteins, the mitochondrial genome and is where the tri-carboxylic acid (TCA) cycle takes place 4.

Within cells, individual mitochondria are largely interconnected, forming what are termed mitochondrial networks. These networks are often at highest density around the nucleus with projections from the main mass towards the edges of the cytoplasm 5. Within these networks, mitochondria are able to undergo fission and fusion. Fission (separation), and fusion (joining) are processes often controlled by stress within cells to tolerate the components of damaged mitochondria or to allow disposal and recycling of proteins via mitophagy in lysosomes 6,7. Mitochondrial networks can be altered in response situations such as increased ROS signalling 8. Therefore, fission, fusion and mitophagy are not only important to regulate the mitochondrial compartment but to respond to the whole state of a cell.

\subsubsection{Mitochondrial Genome}

The endosymbiotic theory states that an $\alpha$-proteobacteria was taken up by a more complex host cell leading to the generation of a eukaryotic cells with mitochondria 6,9,10. However, the type of host cell is still disputed 10 . Nevertheless, this process is believed to be the reason why mitochondria have a double membrane and their own genome 9-11. 
The mitochondria genome is a 16 kilobase circular plasmid type DNA located inside the mitochondrial matrix 12. This circular genome encodes for key proteins that are required to successfully generate ATP via the electron transport chain by oxidative phosphorylation. Mitochondrial DNA encodes for the 7 subunits of NADH dehydrogenase, cytochrome $b$, three cytochrome c oxidase subunits and two subunits of ATP synthase 13-16. Alongside these integral proteins, mtDNA also encodes for its own ribosomal and transferRNAs (tRNA) 13-16.

Replication of mitochondrial DNA is still under investigation 17. However, the basic understanding has been that replication occurs using a "strand-displacement model" 17. Double stranded mitochondrial DNA is composed of a heavy strand and a light strand labelled due to their differences in guanine and thymine nucleotide composition 12,17. The strand displacement model describes separation of strands generating an origin of replication at the displacement-loop (D-loop) location in the control region of mtDNA. DNA is replicated in one direction on one circular strand and before completion, replication is started on the other strand while it is displaced to generate two copies of double stranded circular mtDNA similarly to nuclear DNA replication 12,17.

Cells contain multiple copies of the mitochondrial genome that are individually replicated and, similarly to nuclear DNA, can contain polymorphic differences 18,19 . Presence of different alleles within a cell or organism is termed heteroplasmy 18,20-22. Originally, heteroplasmy was believed to occur in around $1 \%$ of genomes, however with improved molecular techniques, the estimated occurrence is now around 0.2\% 18,21. Variant alleles can be selected for during replication which can result in mitochondrial diseases related to deleterious sequence mutations 22. These mutations, when present in women, can also be inherited by their offspring since mtDNA is maternally inherited 22 .

Interestingly, portions of the complete mitochondrial genome have been inserted into the nuclear genome multiple times, generating nuclear sequences of mitochondrial origin, or NUMTs 23,24. These sequences have been conserved, however they have mostly neutral effects on host biology with only 5 known cases of diseases caused by NUMTs 23,24. 


\subsubsection{Energy Metabolism}

Both nuclear DNA and mitochondrial DNA encoded proteins function together inside the mitochondria to execute the two most important metabolic processes in the mitochondria, the TCA cycle and oxidative phosphorylation.

The TCA cycle converts acetyl-CoA into several different molecules sequentially to shuttle electrons, via electron carriers such as NAD+ and FAD, to the electron transport chain 25 . These reactions take place inside the mitochondrial matrix, inside the inner membrane 25. Acetyl-CoA is natively obtained by breaking down glucose to pyruvate through glycolysis and conversion of pyruvate to acetyl-CoA by pyruvate dehydrogenase 25. All enzymes functioning in the TCA cycle are encoded in the nuclear DNA however the same cannot be said for complexes used in oxidative phosphorylation 25.

While glycolysis can generate small amounts of ATP, oxidative phosphorylation via the electron transport chain (ETC) is the greatest oxygen dependent ATP generator 1. There are 5 multi-protein complexes involved in ETC that span the inner membrane, and the majority of proteins in these complexes are encoded by the nuclear genome (Fig 1.1) 25,26. However, each complex - with the exception of complex II - has at least one component encoded on the mitochondrial genome 27,28. Complex I, also known as NADH dehydrogenase, is encoded by the mitochondrial genome as firstly reduces $\mathrm{NADH}$ to NAD+ to shuttle hydrogen to the inter membrane space 9,14,15,25,27. Complex II, also known as succinate dehydrogenase, is also part of the TCA cycle and is encoded by nuclear DNA 25. Complex II converts succinate to fumarate using $\mathrm{FADH}_{2}$ and together with Complex I shuttles electrons to Co-enzyme Q (CoQ) 25. Cytochrome b is encoded by the mitochondrial genome and forms part of complex III which reduces CoQ to shuttle electrons to cytochrome c (Cyt c) 13,25. Cytochrome $\mathrm{c}$ is then reduced by cytochrome c oxidase, also known as Complex IV, and the electrons are used with oxygen to make water 13,25. The three subunits of Complex IV are also encoded by the mitochondrial genome alongside two subunits of Complex V, or ATP synthase 16,25. Both Complex III and IV shuttle hydrogen into the inner membrane space to generate proton-motive force to drive ATP production via ATP synthase, or complex V (Fig 1.1) $1,16,25,26$. Shuttling electrons through the TCA and electron transport chain also results in 


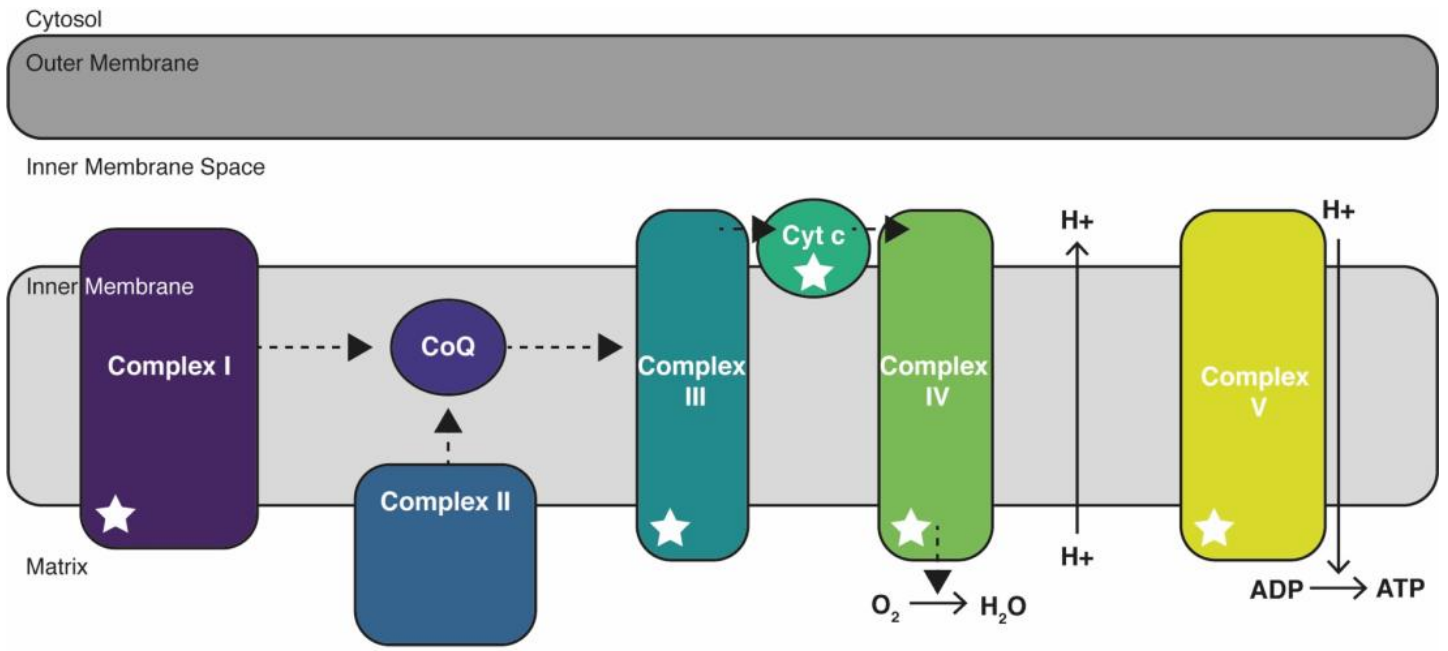

Figure 1.1: Complexes of the Electron Transport Chain.

Complexes of the electron transport chain (ETC) are in the inner membrane of the mitochondria. There are 5 key complexes: Complex I, NADH dehydrogenase; Complex II, Succinate dehydrogenase; Complex III, Cytochrome b; Complex V, Cytochrome c oxidase; Complex V, ATP synthase. The direction of electron movement through the ETC are shown by black arrows with dotted lines. Electrons move from Complex I and II to Coenzyme Q (CoQ) to Complex III to Cytochrome c (Cyt c) to Complex IV where they are used to make water with hydrogen and oxygen. Complex I, III and IV shuttle hydrogen from the matrix to the intermembrane space to generate proton-motive force that drives ATP production by Complex V. Complexes that include proteins encoded by the mitochondrial genome are marked with a white star. Adapted with permissions from Bajzikova et al., 2018. 
production of reactive oxygen species (ROS) 29,30. ROS are molecules that can cause oxidative stress such as damaging DNA and proteins inside cells 30. The balance of ROS is tightly regulated within cells to prevent induction of cell death, for example via apoptosis 2 .

Oxidative phosphorylation requires oxygen to make ATP, however when oxygen is not present, $\mathrm{NADH}$ is reduced to $\mathrm{NAD}+$ by lactate dehydrogenase to convert pyruvate to lactate in the cytoplasm 31. The NADH used in this reaction, rather than being regenerated by the TCA cycle, is regenerated by glycolysis itself 31 .

\subsubsection{Biosynthetic Pathways}

Mitochondria are not just important for energy metabolism in cells, they are also involved in several biosynthetic pathways within cells. These pathways are important for cell function as well as cell proliferation and division. Some examples of these pathways include lipid biosynthesis, synthesis of amino acids, and synthesis of nucleotides 32 .

Mitochondria are also important for carrying out de novo synthesis of amino acids such as glutamine, proline and aspartate 32. For example, $\alpha$-ketoglutarate from the TCA cycle can be used to synthesis glutamine which can be used as a precursor for many other amino acids required by cells 32 . In the reverse pathway, additional glutamine can also be converted to $\alpha$-ketoglutarate which can be used in the TCA cycle 32.

The ETC of mitochondria is highly important for nucleotide production in cells. Pyrimidine synthesis for use in thymine and cytosine production relies on an enzyme called dihydroorotate dehydrogenase (DHODH) 26,32. DHODH can produce orotate when it is able to reduce Coenzyme Q in the inner mitochondrial membrane 32. Therefore, a functioning ETC allows electrons to be continually shuttled through Coenzyme Q and allows DHODH to function 32.

\subsubsection{Iron Metabolism}

Mitochondria also have an important role in metabolising iron in cells. Free iron in the body and in cells can be very toxic 33. A complex array of processes has evolved to regulate this toxicity and utilise the iron as much as possible in biological processes. Iron can be stored both endogenously in cells and externally in the extracellular 
environment such as blood 33. For example, transferrin commonly chelates iron and carries it throughout the body 33. Transferrin and its receptor also provide a common mechanism for iron transport into cells, where iron can then be stored in ferritin 33 .

In addition to storage, free iron can be removed by utilisation through mitochondrial associated processes such as heme production and iron-sulfur cluster synthesis 32 . A large number of proteins located in the mitochondria complex together to form ironsulfate cluster machinery, which assemble free iron and sulfur atoms together in the clusters 34 . Heme is synthesised by eight steps that require enzymes from both the mitochondria and the cytosol 34. One key mitochondrial enzyme, ferrochetalase even requires an iron-sulfur cluster to function 34. Iron-sulfur clusters and heme are then utilised in several proteins within the mitochondria including aconitase and several ETC complexes for their correct formation 34. Heme can also be used in gene regulation for cells such as erythroblasts 35 . Storing and enzyme utilisation are integral in maintaining iron homeostasis within cells and mitochondria have a key role in this maintenance.

\subsubsection{Mitochondrial Injuries}

Mitochondria have such an important role in the energetic state of cells that mutations and dysfunctions are implicated in many metabolic related disorders. These disorders often manifest in highly active tissues such as neural, cardiac, and muscular tissues 36.

\subsubsection{Cancer Phenotype}

One of the most highly studied conditions related to mitochondrial dysfunction is cancer. Cancer is a culmination of mutations, often to DNA, leading to altered proliferative, metabolic and functional state 37. Classically, cancer cells were believed to have undergone a transition from using anaerobic glycolysis and oxidative phosphorylation in their pre-cancer state, to using aerobic glycolysis as their main production of ATP, termed the Warburg effect 37. Aerobic glycolysis is inefficient at producing ATP compared to oxidative phosphorylation, however it involves primarily converting pyruvate to lactate, even in the presence of oxygen, which can be used to create other biosynthetic molecules that cells require to maintain high proliferative rates 37. More recently, highly glycolytic cells that do not contain mitochondrial DNA and therefore, cannot perform oxidative phosphorylation, po cells, have been used to show that a basal level of oxidative phosphorylation is required for cancer cells to form 
tumours in mice 29,38,39. With research illuminating requirements for mitochondria within cancer development and tumorigenicity, it is clear there are complex relationships between mitochondria and energetic requirements of cancer cells.

\subsubsection{The po phenotype}

An extreme mitochondrial injury can be found in the form of $\rho$ o cells, cells that no longer have mitochondrial DNA 26,38-42. These cells are most commonly created by treatment with low-dose ethidium bromide long-term in culture 40,43. Ethidium bromide intercalates with double stranded DNA, including the mitochondrial genome, preventing replication 40,44 . Therefore, with every subsequent division in culture a treated cell dilutes the mitochondrial genome until there are no detectable molecules left 40,44. Ethidium bromide treatment can also damage the nuclear genome and these mutations cannot be controlled 40. Alternatively, a mitochondrial targeted endonuclease has been used for more rapid degradation of the mitochondrial genome to generate $\rho o$ cells, which does not produce the off-target nuclear genome effects seen in ethidium bromide treatment 40 .

The metabolism of po cells is drastically altered in response to the loss of mitochondrial DNA. The proteins encoded in the mitochondrial genome are critical for formation of functional ETC complexes, and hence oxidative phosphorylation for ATP generation 1316,25. Without the mitochondrial genome, complexes I, III, IV and part of complex V cannot assemble, leading to complete collapse of the electron transport chain 26,39 (Fig 1.2). Loss of these complexes prevents NAD+ regeneration via ETC, therefore cells increase their glycolytic capacity and prioritise conversion of pyruvate to lactate to compensate 32. Pyruvate is therefore, not only being used to produce high levels of ATP inefficiently, but still required for other pathways such as amino acid synthesis 32. Therefore, $\rho$ o cells die in culture without pyruvate supplementation.

Characteristically, po cells also require uridine supplementation in their media and this is for use in synthesising pyrimidines for nucleotides 32 . DHODH is a crucial enzyme in this process, however it relies on reduction of Coenzyme $Q$ in the electron transport chain 26,32. CoQ is still present in po cells, though the lack of complex III traps CoQ in its fully reduced state from complex II in the TCA cycle, leading to loss of DHODH 
Inner Membrane Space

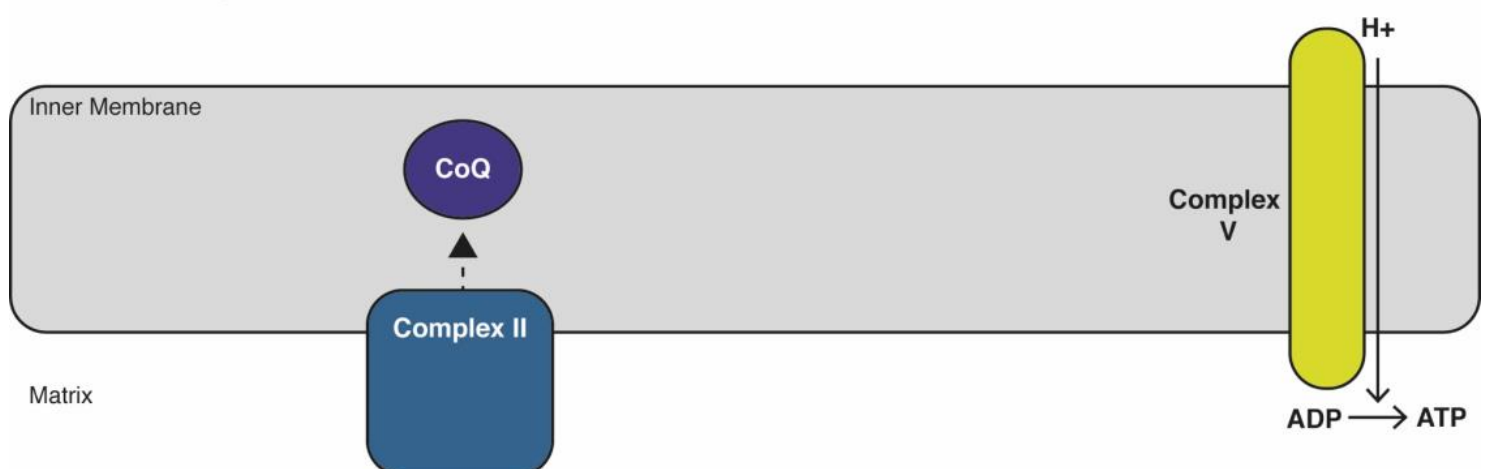

Figure 1.2: Electron Transport Chain complexes in po cells.

Complexes of the electron transport chain (ETC) remaining after loss of mtDNA. Complex II, Succinate dehydrogenase and a subunit of Complex V, ATP synthase. The direction of electron movement through the ETC are shown by black arrows with dotted lines. Electrons move from Complex II to Coenzyme Q (CoQ) where it remains fully reduced due to lack of Complex III. No hydrogens are shuttle into the intermembrane space leading to lack of proton-motive force that drives ATP production by Complex V. Adapted with permissions from Bajzikova et al., 2018. 
function 26,32. Uridine supplementation alleviates the requirement of DHODH in pyrimidine synthesis allowing cells to continue proliferating 32.

\subsubsection{Deferoxamine}

Iron is important for the function of several cellular proteins and the development of cells such as erythroblasts 34,45,46. Lack of iron clinically presents in anaemia, which can ultimately lead to cell death via loss of heme and increased oxidative stress 47 . At a subcellular level, lack of iron also results in dysfunctional and incompletely formed enzymes including those such as ferrochetalase and electron transport chain complexes

34. Deferoxamine is an iron chelator, modelled from organisms that produce siderophores to reduce availability of iron in their host cells 48,49. It is often used in response to iron overload conditions where iron becomes toxic 49,50. Iron chelators could induce mitochondrial injury when over used, and are being considered for use in treatments of cancers and atherosclerosis 48 .

\subsubsection{Cisplatin}

Cisplatin is a chemotherapeutic drug commonly used in the treatment of several cancers such as bladder, lung and ovarian cancer 51. Cisplatin is known to cause crosslinking of purine bases in double stranded DNA 51. This crosslinking prevents activity of DNA repair pathways and ultimately leads to DNA damage that induces cells to undergo apoptosis 51. Cisplatin also induces oxidative stress that damages not only DNA but proteins within cells and has been implicated in reduction of mitochondrial membrane potential 51. Reduction in mitochondrial membrane potential can lead to increased permeability and induce release of cytochrome $\mathrm{c}$ to trigger the intrinsic apoptotic pathways of cell death 3,51 .

\subsection{Mitochondrial Transfer Phenomenon}

\subsubsection{Origins}

In 2004, organelles were demonstrated to move between rat cells via tubular connections 52. These tubular connections were named tunnelling nanotubules and the organelles transferred between the cells included endosomes, lysosomes, and mitochondria 52. The first visual demonstration was in 2006, using both images and video, showed that mitochondrial networks within human mesenchymal stem cells 
(hMSC) localized towards po cells and induced division of $\rho$ o cells in metabolitedeprived conditions 42. It was also shown that mitochondrial function was rescued and mtDNA polymorphisms of the donor hMSC were acquired by the A549 po cells 42. These two studies are the corner-stones of mitochondrial transfer.

Theories of mitochondrial transfer culminate in three key proposed mechanisms: tunnelling nanotubules, extracellular microvesicles and exosomes, or by fusion of cells (Fig 1.3) 41,53. The specific signals that drive this phenomenon between cells are still not known. However, mitochondrial transfer has been seen in response to stressors such as chemotherapies, oxidative stress by ROS, deprivation of pyruvate and uridine in po cells 26,39,61-64,42,54-60. These situations allude to mitochondrial transfer improving the metabolic states allowing better recovery from injury 42,57-63. However, there is some evidence to suggest that damaged mitochondria may be transferred to healthy cells for aid in mitophagy, or disposal of the damaged organelles 54,65. Subsequent and current research in the field, has not investigated if the process can occur independently from damage-inducing treatments, and therefore if it is a ubiquitous process.

\subsubsection{Examples of Mitochondrial Transfer}

Mitochondrial transfer is most commonly explored using cancer cell lines and injured cell models. Cancer cell model tend to focus their efforts on the phenomenon in response to chemotherapeutics 57,59,61,63,66. Emerging research, and part of the rationale for this thesis, is beginning to delve into the context of bone marrow and is represented by three key studies.

The first published study investigated mitochondrial transfer in human acute myeloid leukemia (AML) cells 61. Primary human AML cells were cultured in vitro with murine bone marrow stromal cells (BMSC), human BMSC or transplanted into the bone marrow of NSG mice as a xenograft model. Transfer of mitochondria was detected, using transfer of fluorescent proteins in vitro and a human-mouse mtDNA distinguishing PCR with a nuclear control for the in vivo xenograft, in each of these conditions. The transfer of mitochondria increased the resistance of the AML cells to chemotherapeutic treatments such as cytarabine or doxorubicin 61 . 
Tunnelling Nanotubules

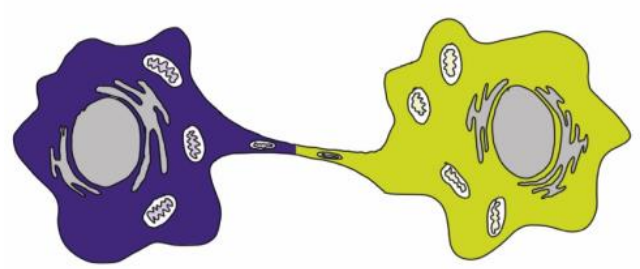

Microvesicles/Exosomes
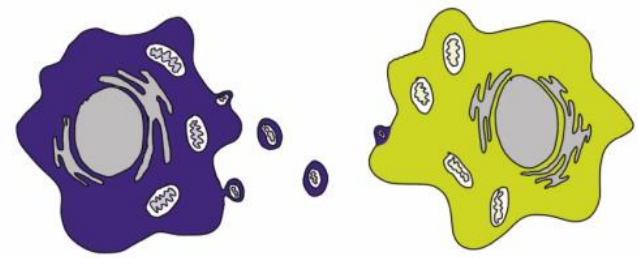

Cell Fusion
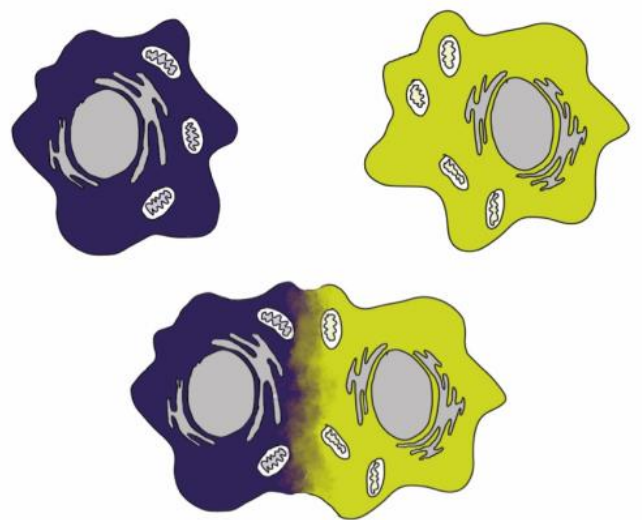

Figure 1.3: Postulated mechanisms of Mitochondrial Transfer

Three main mechanisms for transferring mitochondria between cells have been proposed. The first is via cytoplasmic connections called tunneling nanotubules. The second is via containment in microvesicles or exosomes which fuse with the membrane of other cells. The third is uptake of mitochondria via cell fusion where two cells become one. Adapted from Spees et al., 2016. 
Human AML cells were shown in a second study to receive mitochondria from BMSC both in vitro and via in vivo xenograft of human AML cells into a highly immuecompromised NSG mouse model 62. Using fluorescent dyes retained in the mitochondria, transfer occurred in vitro via tunnelling nanotubules and appeared to be stimulated by ROS generated by an enzyme, NADPH oxidase- 2 generates superoxide. The study indicated that increased superoxide signalled the BMSC to donate mitochondria to the AML cells in co-culture and in the in vivo model 62.

BMSC have been shown a third time to donate mitochondria to cancer cells from the bone marrow, this time into multiple myeloma (MM) cells 63. Primary human MM cells were shown to produce tunnelling nanotubules with human BMSC in a co- culture. The mitochondria of the BMSC were stained with MitoTracker dyes. Again, human cells were transplanted into immune compromised mice similar to the first two studies. A human-mouse mtDNA specific endpoint PCR was performed on the MM cells to show that they had acquired murine mtDNA. Bortezomib, a proteasome inhibitor used therapeutically was used to show that stress does increase mitochondrial transfer in these conditions, corroborating the previous study. However, they also implicated the cell surface expressed protein CD38: expression was newly found on BMSC after coculture with MM cells; and knockdown of CD38 reduced the occurrence of mitochondrial transfer 63.

Rho zero cells provide an extreme injury that induces transfer and have been shown in multiple studies. A study in 2015 showed transfer into both murine breast cancer $4 \mathrm{~T} 1 \rho$ o

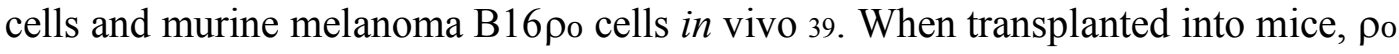
cells were initially unable to form tumors. Most notably for both cell lines mtDNA was acquired from the host mice cells, following transplantation. The acquisition of mtDNA corresponded with functional recovery of respiratory complexes within mitochondria, recovered mitochondrial membrane potential as shown by ethidium bromide staining and an induction of tumour formation 39.

Where most transfer of mitochondria occurs from healthy cells into damaged cells to aid their recovery, a study published in 2015 showed mesenchymal stem cells (MSC) sending extracellular vesicles containing mitochondria to macrophages for destruction via mitophagy 54. Depolarised mitochondria were found in microvesicles from MSCs using electron microscopy and using mitochondrial labelling dyes that were present in 
both primary mouse macrophages and RAW 264.7 cells co-cultured with them. Interestingly, these microvesicles were also able to reduce inflammation and immune activation in mice that were exposed to silica in the lungs 54. Similarly, optic nerve cells have been shown to expel their damaged mitochondria which are taken up by glial support cells and degraded via mitophagy 65.

\subsubsection{Techniques to identify transfer of mitochondria}

The studies previously described highlight only a few of the numerous techniques used to detect transfer of mitochondria. However, many techniques used to identify mitochondrial transfer have limitations that should be considered. Most commonly used techniques are microscopy based and often use fluorescent dyes such as MitoTracker TM which rely on mitochondrial membrane potential to contain the dye within the mitochondria 67,68. Mitochondrial membrane potential can fluctuate over time and thus these dyes are prone to leakages especially when not used correctly 67,68 . Therefore, when research details movement of fluorescent dye in vesicles or moving across tunnelling nanotubules it can be difficult to ascertain a physical mitochondrion is present.

Staining the mitochondrial network with agents such as ethidium bromide can be used to identify transfer when working with po cells 39,68 . po cells lack mitochondrial DNA and have a low membrane potential, and therefore do not stain with ethidium bromide 39. As a result, ethidium bromide can be successfully used to visualise the recovery of po cell mitochondria 39 .

Alternative methods include fluorescent mitochondrial proteins, these can be stably produced via lentiviral or plasmid transfection methods in culture, or even using cells from mice that stably express fluorescent proteins targeted to the mitochondria. For example, the mito-dsRed mouse expresses the dsRed fluorophore, linked to a mitochondrial-targeting signal, from a transgenic promoter 41 . These fluorescent proteins are most commonly transcribed from the nucleus of the cell of origin, and localise to the mitochondria. However, if a mitochondrion is transferred into another cell that does not encode the gene for the fluorescent protein, when the original fluorescent proteins are turned over after incorporation into the recipient network, 68 the signal is lost. 
Molecular methods are less commonly used for detection of mitochondrial DNA transfer; however, this is beginning to change. Examples include leveraging interspecies differences in mitochondrial DNA sequences, which is most commonly used for detecting transfer between human and mouse mitochondria in xenograft models 61-63. Simple sequencing approaches can also be used when examining transfer into po cells the lack of background DNA means that all mtDNA has come from transferred mitochondria 39. Additionally, more specific polymorphisms in mtDNA sequences between the same species can be leveraged using quantitative measures such as qPCR. A specific assay can be designed to selectively amplify the target mtDNA sequence determined by a single polymorphism in a background of mtDNA without that polymorphism 69. The design includes an allele specific primer for the target, a blocking primer for the off-target sequence with a 3' phosphate group, a common reverse primer and a fluorescent probe that binds each molecule (Fig 1.4) 69.

Interestingly, a technique can also be utilised that combines both visual and molecular evidence for transfer of mitochondria. This technique utilises padlock probes that are specific for a mtDNA genotype target. Once hybridised to the specific target, rolling circle amplification occurs from the padlock probe. This polymerase-based DNA amplification then uses a fluorescent probe that hybridises to the amplified DNA to visualise mitochondrial genomes using fluorescence microscopy. This can be done within fixed cells or tissue sections in situ 70. 


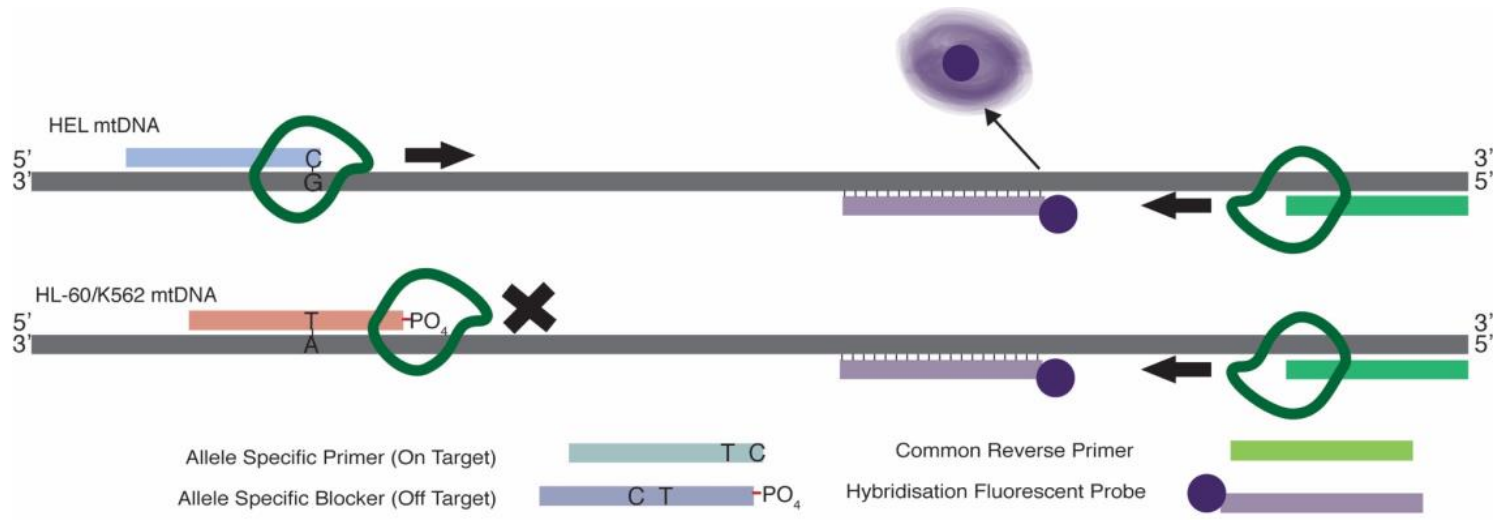

\section{Figure 1.4: Allele-Specific-Blocker qPCR Model}

Schematic depicting the designed ASBqPCR for detecting HEL mtDNA in a HL-60 or K562 DNA background. The background polymorphism is bound by a blocker primer with a 3 ' phosphate group preventing extension by DNA polymerase, preventing release of fluorescence from the probe downstream. Both on- and off-target templates are amplified using the common reverse primer. The target sequence is bound by a specific primer which allows DNA polymerase extension and release of fluorescence read by the qPCR machine. Adapted from Morlan et al., 2009. 


\subsection{Mitochondrial Transfer in Human Bone Marrow}

\subsubsection{Cells of Interest in Bone Marrow}

\subsubsection{Mesenchymal Cells}

The bone marrow is composed of several different cell types including mesenchymal cells, and cells of lymphoid or myeloid origin including erythroid lineages 71. Mesenchymal stem cells can self-renew in the bone marrow and differentiate into many cells such as adipocytes, osteoblasts and chondrocytes (Fig 1.5.B) 71. Mesenchymal cells are believed to drive differentiation and function and are known to secrete large numbers of factors and microvesicles into their surrounding environment 53. Interestingly, mesenchymal cells have been shown to partake in cell fusion to rescue damaged cells in their environment 53 . These cell are one of the main candidates for donors of mitochondria to damaged or cancerous cells, most commonly shown through tunnelling nanotubules 42,54-59,61-63.

\subsubsection{Lymphoid cells}

Haematopoietic stem cell progenitors differentiate into two key lineage progenitor cells, common lymphoid progenitor cells and common myeloid progenitor cells (Fig 1.5.A) 71. Lymphoid progenitors maintain the lymphoid compartment of the bone marrow by differentiating into B- and T- lymphocytes and natural killer cells, which function in the body as the part of the innate and adaptive immune systems 71 .

\subsubsection{Myeloid Cells}

Common myeloid progenitor cells generate the myeloid compartment by differentiating into macrophages and granulocytes such as neutrophils and basophils which also form part of the innate immune system 71. Common myeloid progenitors also have the ability to differentiate to megakaryocytes and erythrocytes (Fig 1.5.A).

\subsubsection{Erythroid Cells}

Erythroid cells are generated by common myeloid progenitor cells and differentiate through their own process called erythropoiesis into red blood cells (RBC) that circulate in our blood to carry oxygen throughout the body 71-76. Erythropoiesis begins with the generation of pro-erythroblasts that asymmetrically divide into basophilic, 
orthochromatic and polychromatic stages sequentially 73,76. After this point the cells extrude their nucleus and mitochondria to become reticulocytes and finally RBCs (Fig 1.5.C) 73,76 .

Differentiation of erythroid cells relies heavily on mitochondrial metabolism, in particular iron-sulfur cluster and heme production 34,35,47. Iron-sulfur clusters help assembly of enzymes involved in oxidative phosphorylation and also enzyme involved in heme production 34,45 . Heme serves two main functions, to direct gene expression for differentiation and enzymes to make haemoglobin, and to be directly involved in making the haemoglobin, the oxygen carrier in RBCs 34,35 .

Iron is important in the erythroid lineage. Toxicity of iron to erythroid cells can be seen in iron overload conditions 33. Iron overload prevents differentiation of erythroid compartment into mature cells, leaving the cells largely immature and unable to differentiate into RBCs 75,77. It also increases cell death via apoptosis 75,77 . Deficiency in erythropoiesis, which may be caused by resistance to erythropoietin, can lead to anaemia, or low levels of iron 75,78 .

Erythroid cells have not been studied in mitochondrial transfer research, however there are aspects about these cells that make a compelling model. They express CD38 at early pro-erythroblast stage of differentiation which has been implicated in previous bone marrow mitochondrial transfer research 79,80. CD38 has multiple enzyme functions such as ADP cyclising as well as ADP and NAD hydrolysis 80. CD38 is also believed to have roles in proliferation and differentiation of cells 80 . Haematologists and pathologists have long observed inter-erythroid cytoplasmic bridges in bone marrow aspirate smears 81,82. Additionally, these bridges are seen most often between polychromatophilic and orthochromatic staged erythroblasts, with significantly less frequent visualisation between erythroblasts earlier in differentiation (personal communication, Andrew Hall, MIMR, 2016). The function of these connections is not known currently but may be used to transfer cellular components such as mitochondria. 


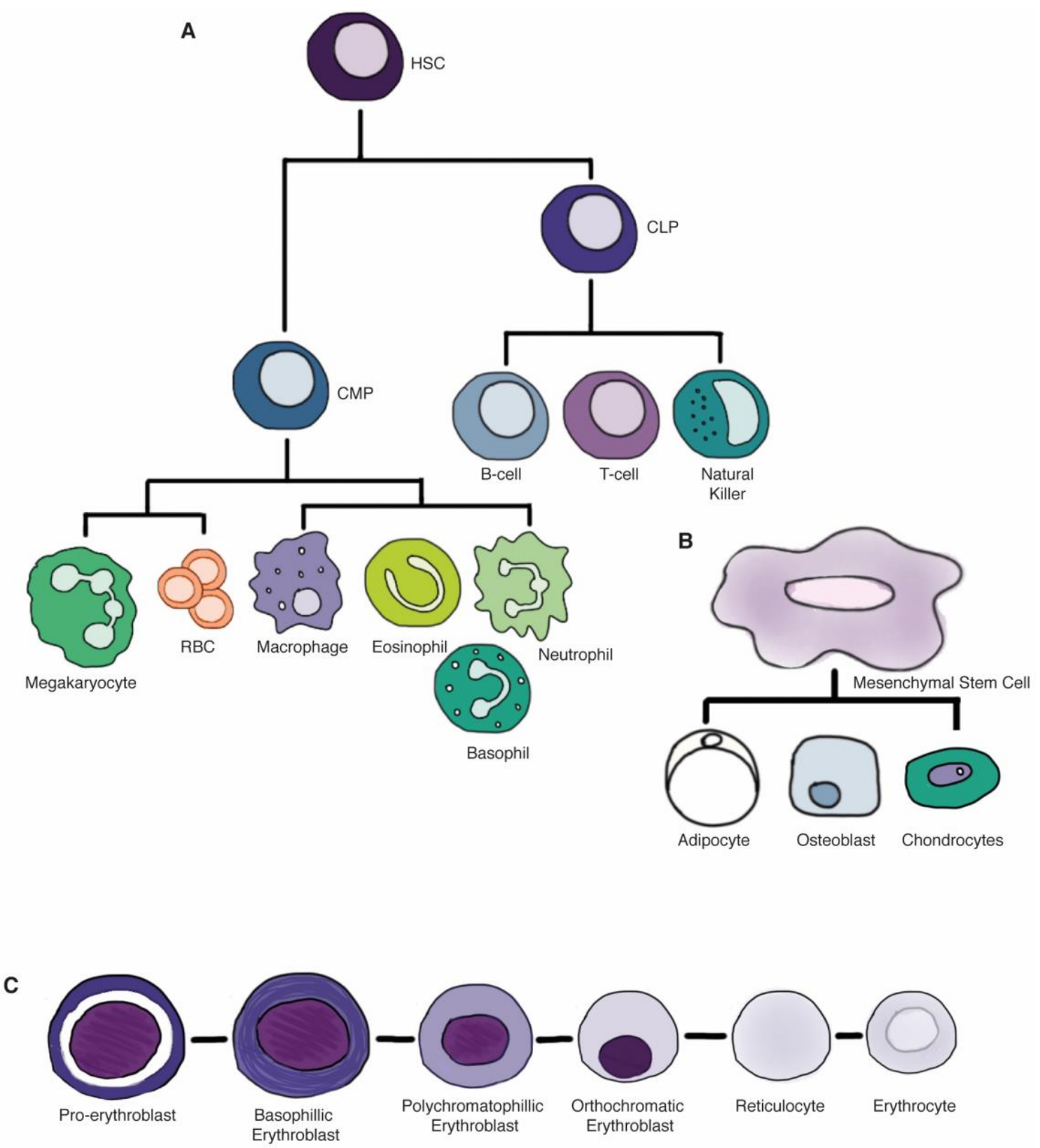

Figure 1.5: Hematopoietic, Mesenchymal and Erythroid Differentiation.

(A) Schematic of Hematopoietic stem cell (HSC) differentiation through common lymphoid progenitor (CLP) or common myeloid progenitor (CMP) and differentiation into subsequent cell types. (B) Mesenchymal stem cell differentiation in the bone marrow. (C) Diagram of erythropoiesis and morphological presentation of erythroid stages under May-Grunwald Giemsa stain. (A\&B) Adapted with permissions from Reagan \& Rosen 2015. (C) Adapted from Liu et al., 2015. 


\subsubsection{Models of Mitochondrial Transfer between Human Erythroblasts}

\subsubsection{In Vitro and Ex Vivo Co-cultures}

Investigation into transfer of mitochondria requires co-culture of cells where one cell is a donor of the mitochondria and one cell is the recipient. Erythroblasts can be modelled in vitro by three human cancer cell lines. HEL cells are from a human erythroleukemia 83, K562 cells are a chronic myeloid leukemia with erythroid 84,85 characteristics and HL-60 cells which are an acute myeloid leukemia 43,84,86. HL-60 cells also have a po derivative line which allows for exploration in that context of mitochondrial transfer 43.

Cell lines will always have limitations as model systems. While human bone marrow contains a lot of erythroid cells, obtaining such bone marrow to work with is problematic. Healthy people do not want to donate bone marrow, and unwell people having bone marrow aspiration are likely to have defects in mitochondrial function. Ex vivo co-culture is an important step for this research to indicate the likelihood that transfer of mitochondria can occur in vivo in humans.

A recent paper published gives the opportunity to examine erythroid co-culture, and the potential for transfer, ex vivo 87. Using a 17-day culture method, peripheral blood mononuclear cells (PBMCs) can be differentiated into all stages of erythroblasts. The complete culture media contains key components, stem cell factor (SCF), interleukin-3 (IL-3), erythropoietin (EPO), dexamethasone and $\beta$-estradiol. The first phase of culture enlists addition of extra dexamethasone every 48 hours up to day 10 and from then on, extra of both dexamethasone and $\beta$-estradiol are added until day 1787.

\subsubsection{In Vivo Bone Marrow Transplants}

In vivo experiments are the pinnacle of investigating this phenomenon in humans and exploring bone marrow erythroid cells for transfer of mitochondria has a unique translational aspect. Bone marrow from healthy donors is regularly transplanted into patients as part of treatment for cancer, essentially providing an in vivo co-culture condition between donor and recipient bone marrow cells 88 . Bone marrow transplants can occur into patients who have undergone reduced intensity conditioning, myeloablative or non-myeloablative conditioning 89,90. Each transplant type provides 
different levels of co-culture for mitochondrial transfer, for example reduced intensity conditioning often results in residual patient bone marrow cells 89,90. Erythroid cells express surface proteins such as blood group antigens 91. These proteins provide a novel mechanism to separate donor cells from recipient bone marrow after co-culture. If mtDNA SNPs can be identified between bone marrow donor and bone marrow recipient, then mitochondrial DNA can be analysed, to determine whether mitochondrial transfer happens in people, an exciting prospect for this research. 


\subsection{Aims and Objectives}

\subsubsection{Aims}

There are several compelling aspects to erythroid cells that indicate mitochondrial transfer may occur between them. For example, early erythroid cells express CD38 which has recently been implicated in transfer of mitochondria within the bone marrow 63,79. Additionally, inter-erythroid cytoplasmic bridges have been seen between midstage differentiated erythroid cells (personal communication, Andrew Hall, MIMR, 2016). Erythroid cells rely on mitochondria for their differentiation via heme production and iron metabolism in a complex relationship 34. Interestingly, transfer of mitochondria has not been studied in erythroid cells before. Therefore, it is not known if these cells can participate in this phenomenon. This thesis aims to conduct studies in vitro and ex vivo that will indicate whether transfer of mitochondria could be seen in vivo in the unique setting of bone marrow transplants. This would provide future therapeutic targets in cancer treatment or other diseases related to bone marrow and erythroid cells.

\subsubsection{Objectives}

1. To develop an allele-specific qPCR assay to detect mtDNA transfer between erythroid cells after co-culture.

2. To investigate transfer of mitochondria between a po erythroid cell line that lacks mtDNA, and healthy donor erythroid cells in vitro.

3. To determine if healthy erythroid cells can donate mitochondria to injured erythroid cells in vitro.

4. To assess transfer of mitochondria between healthy PBMC derived erythroblasts and deferoxamine treated PBMC derived erythroblasts ex vivo. 
2 Materials and Methods 
Table 2.1: List of Laboratory Consumables

\begin{tabular}{|c|c|}
\hline Product & Supplier/Distributer \\
\hline $0.2 \mathrm{~mL}$ SnapStrip ${ }^{\circledR}$ II PCR Tubes & SSIbio \\
\hline $\begin{array}{c}\text { BD Vacutainer }{ }^{\circledR} \text { K2E EDTA } 10 \mathrm{~mL} \\
\text { Tubes }\end{array}$ & BD Biosciences \\
\hline $\begin{array}{c}\text { Corning }{ }^{\circledR} \text { Costar }{ }^{\circledR} \text { Serological Pipettes } \\
-5 \mathrm{~mL}, 10 \mathrm{~mL}, 25 \mathrm{~mL}\end{array}$ & Corning Incorporated \\
\hline Corning ${ }^{\circledR}$ Microtubes $1.7 \mathrm{~mL}$ & Axygen Scientific \\
\hline Cryovials & Greiner Bio-One GmbH \\
\hline CytoFunnel® with White Filter Cards & Thermo Fisher Scientific, New Zealand \\
\hline $\begin{array}{l}\text { Deckglaser Microscope Cover Glasses } \\
\qquad 22 \times 22 \mathrm{~mm}\end{array}$ & Lab Supply \\
\hline $\begin{array}{c}\text { Falcon }{ }^{\mathrm{TM}} 5 \mathrm{~mL} \text { Polypropylene Round- } \\
\text { Bottom Tube with Cap }\end{array}$ & BD Biosciences \\
\hline $\begin{array}{c}\text { Falcon }^{\mathrm{TM}} \text { 5mL Polystyrene Round } \\
\text { Bottom Tube }\end{array}$ & BD Biosciences \\
\hline $\begin{array}{l}\text { Falcon }^{\mathrm{TM}} 5 \mathrm{~mL} \text { Polystyrene Round } \\
\text { Bottom Tube with Cell Strainer cap }\end{array}$ & BD Biosciences \\
\hline $\begin{array}{l}\text { Falcon }{ }^{\mathrm{TM}} \text { Plates }-6 \text { well flat, } 24 \text { well } \\
\text { flat, } 96 \text { well U bottomed }\end{array}$ & BD Biosciences \\
\hline Falcon ${ }^{\mathrm{TM}}$ Tubes $-15 \mathrm{~mL}, 50 \mathrm{~mL}$ & BD Biosciences \\
\hline Glass Pipettes & Thermo Fisher Scientific, New Zealand \\
\hline KJ619-1A 1mL Pasteur Pipette & Interlab \\
\hline $\begin{array}{c}\text { MicroAmp }{ }^{\circledR} \text { Optical 384-Well Reaction } \\
\text { Plate with Barcode }\end{array}$ & Applied Biosciences, Life Technologies \\
\hline MicroAmp ${ }^{\circledR}$ Optical Adhesive Film & Applied Biosciences, Life Technologies \\
\hline $\begin{array}{c}\text { MicroAmp }{ }^{\mathrm{TM}} \text { Fast } 96 \text {-well Reaction } \\
\text { Plate }(0.1 \mathrm{~mL})\end{array}$ & Applied Biosciences, Life Technologies \\
\hline $\begin{array}{c}\text { MicroTouch }{ }^{\circledR} \text { Dermaclean }{ }^{\circledR} \text { Latex } \\
\text { Gloves }\end{array}$ & Ansell \\
\hline Nunc EasYFlasks - T25, T75, T125 & Thermo Fisher Scientific, New Zealand \\
\hline Pipette Filter Tips & Axygen Scientific \\
\hline
\end{tabular}




\begin{tabular}{|c|c|}
\hline Product & Supplier/Distributer \\
\hline Pipette Tips $-10 \mu \mathrm{L}, 200 \mu \mathrm{L}, 1000 \mu \mathrm{L}$ & Axygen Scientific \\
\hline Shandon Filter Cards (White) & Thermo Fisher Scientific, New Zealand \\
\hline $\begin{array}{c}\text { Superfrost } \AA \text { Plus Microscope Slides } \\
25 \times 75 \times 1 \mathrm{~mm}\end{array}$ & LabServ \\
\hline Unistik ${ }^{\circledR} 3$ Normal Lancets & Mediray \\
\hline $\begin{array}{c}\text { Acrodisc } \AA \text { Syringe Filters with } 0.22 \mu \mathrm{m} \\
\text { Supor }{ }^{\circledR} \text { Membrane }\end{array}$ & Sigma Alsrich \\
\hline
\end{tabular}

Table 1.2: List of Laboratory Equipment

\begin{tabular}{|c|c|}
\hline Equipment & Manufacturer \\
\hline BD Influx ${ }^{\mathrm{TM}}$ Cell Sorter & BD Biosciences \\
\hline Bio-Rad Gel Tank & BioRad \\
\hline Bio-Rad Powerpac Basic & BioRad \\
\hline Biomedical Freezer $\left(-20^{\circ} \mathrm{C}\right)$ & Sanyo \\
\hline C1000 Touch Thermal Cycler & BioRad \\
\hline Centrifuge & Eppendorf \\
\hline ChemiDoc $^{\mathrm{TM}}$ Touch Imaging System & BioRad \\
\hline Cytek Aurora Spectral Cytometer & Cykek, CA \\
\hline Delta Range PC 8800 Balance & Mettler \\
\hline HeraCell Incubator & Heraeus Instruments \\
\hline HeraSafe Sterile Hood & Heraeus Instruments \\
\hline Liquid Nitrogen & Cryogenic Products Limited \\
\hline LSR- II SPOR Flow Cytometer & BD Biosciences \\
\hline Megafuge $2.0 \mathrm{R}$ & Heraeus Instruments \\
\hline Nalgene $^{\mathrm{TM}}$ Mr Frosty & Thermo Fisher \\
\hline NanoDrop One & Thermo Fisher \\
\hline $\begin{array}{c}\text { Olympus BX51TF Compound } \\
\text { Microscope }\end{array}$ & Olympus, Japan \\
\hline Olympus IX51 Inverted Microscope & Olympus, Japan \\
\hline Pipette & Thermo Scientific and Gilson \\
\hline
\end{tabular}




\begin{tabular}{|c|c|}
\hline Equipment & Manufacturer \\
\hline QuantStudio $^{\text {TM }} 7$ Flex & Life Technologies \\
\hline Refrigerator & Panasonic \\
\hline Shandon Cytospin 4 & Thermo Fisher \\
\hline SUB Aqua 12 Plus & Thermo Fisher \\
\hline Thermo Fisher Pipette Gun & Thermo Fisher \\
\hline Thermomixer Compact & Eppendorf \\
\hline Fltra-Low Temperature High Efficiency & New Brunswick \\
\hline
\end{tabular}

\subsection{Buffers, Reagents and Kits}

Table 2.1.1 List of Reagents

\begin{tabular}{|c|c|}
\hline Reagent & Supplier \\
\hline$\beta$-Estradiol $\geq 98 \%$ & Sigma Aldrich \\
\hline$\beta$-mercaptoethanol 1000X & Gibco, Life Technologies \\
\hline 2x KAPA2G Fast HotStart ReadyMix & Sigma Aldrich \\
\hline $\begin{array}{l}\text { 5X HOT FIREPol }{ }^{\circledR} \text { Probe Universal } \\
\text { qPCR Mix }\end{array}$ & Solis BioDyne \\
\hline Annexin V Binding Buffer 1X & Biolegend \\
\hline Blue Juice Loading Buffer & Invitrogen Life Technologies \\
\hline Cisplatin $\geq 95 \%$ & Cayman Chemical \\
\hline DAPI Viability Stain & Life Technologies \\
\hline $\begin{array}{c}\text { DBL }^{\mathrm{TM}} \text { Dexamethasone Sodium } \\
\text { Phosphate }\end{array}$ & Hospira NZ Ltd \\
\hline $\begin{array}{l}\text { Deferoxamine mesylate salt } \geq 98.5 \% \\
\text { (TLC) }\end{array}$ & Sigma Aldrich \\
\hline $\begin{array}{c}\text { Ambion DEPC-treated Nuclease Free } \\
\text { Water (for sequencing) }\end{array}$ & Thermo Fisher Scientific \\
\hline Dimethyl Sulfoxide (DMSO) & Sigma Aldrich \\
\hline DNA Clean and Concentrator ${ }^{\mathrm{TM}}$ Kit & Zymo Research \\
\hline
\end{tabular}




\begin{tabular}{|c|c|}
\hline Reagent & Supplier \\
\hline $\begin{array}{c}\text { Dulbecco’s Modified Eagle's Media - } \\
\text { high Glucose }\end{array}$ & Sigma Aldrich \\
\hline $\begin{array}{l}\text { Dulbecco's Modified Eagle's Media } \\
\text { (dMEM) }\end{array}$ & Gibco, Life Technologies \\
\hline $\begin{array}{l}\text { Dulbecco’s Phosphate Buffered Saline } \\
\qquad(\mathrm{dPBS})\end{array}$ & Gibco, Life Technologies \\
\hline Eprex ${ }^{\circledR}$ Human Erythropoetin & Janssen \\
\hline Ethanol absolute anhydrous & Pure Science, Porirua, NZ \\
\hline Fetal Bovine Serum (FBS) & Gibco, Life Technologies \\
\hline Glutamax & Gibco, Life Technologies \\
\hline Human Recombinant Stem Cell Factor & Stem Cell \\
\hline HyAgarose $^{\mathrm{TM}}$ & Hydragene \\
\hline IL-3 Recombinant Human Protein & Thermo Fisher Scientific \\
\hline $\begin{array}{c}\text { Invitrogen }^{\mathrm{TM}} \text { Platinum }^{\mathrm{TM}} \text { SuperFi }^{\mathrm{TM}} \text { PCR } \\
\text { Master Mix }\end{array}$ & Invitrogen, Life Technologies \\
\hline $\begin{array}{l}\text { Iscove's Modified Dulbecco's Medium } \\
\text { (IMDM) }\end{array}$ & Life Technologies \\
\hline KAPA HiFi HotStart ReadyMixPCR Kit & KAPABiosystems \\
\hline Kwik-Diff ${ }^{\mathrm{TM}}$ Eosin Solution & Shandon \\
\hline Kwik-Diff ${ }^{\mathrm{TM}}$ Fixative Solution & Shandon \\
\hline Kwik-Diff ${ }^{\mathrm{TM}}$ Methylene Blue Solution & Shandon \\
\hline LabChem DPX Mounting Media & Ajax Finechem \\
\hline Lymphoprep $^{\mathrm{TM}}$ & Axis Shield \\
\hline Ambion Nuclease Free Water (for PCR) & Thermo Fisher Scientific \\
\hline Penicillin Streptomycin & Life Technologies \\
\hline Premium Bleach & Cyclone \\
\hline Propidium Iodide & BD Pharminigen \\
\hline Quick gDNA ${ }^{\mathrm{TM}}$ Microprep Kit & Zymo Research \\
\hline Quick gDNA ${ }^{\mathrm{TM}}$ Miniprep Kit & Zymo Research \\
\hline
\end{tabular}




\begin{tabular}{|c|c|}
\hline Reagent & Supplier \\
\hline $\begin{array}{c}\text { Roswell Park Memorial Institute Media } \\
\text { RPMI-1640) }\end{array}$ & Gibco, Life Technologies \\
\hline Sodium Chloride $\geq 99 \%$ & Sigma Aldrich \\
\hline Sodium Pyruvate $100 \mathrm{mM}$ & Life Technologies \\
\hline SYBR ${ }^{\mathrm{TM}}$ Safe DNA Gel Stain $(10000 \mathrm{x})$ & Invitrogen, Life Technologies \\
\hline TAE Buffer & Invitrogen, Life Technologies \\
\hline Trackit ${ }^{\mathrm{TM}} 100 \mathrm{bp}$ DNA Ladder & Invitrogen, Life Technologies \\
\hline Trypan Blue & Gibco, Life Technologies \\
\hline Ultra-Pure 0.5 M, pH 8.0 EDTA & Invitrogen, Life Technologies \\
\hline UltraComp eBeads & eBioscience Affymetrix \\
\hline Uridine & Sigma Aldrich \\
\hline
\end{tabular}

\subsubsection{Complete Cell Line Culturing Media}

Both RPMI-1640 and DMEM were supplemented with 10\% FBS. Only DMEM was supplemented with $2 \mathrm{mM}$ Glutamax. 1\% Penicillin Streptomycin was omitted from media except after sterile sorts.

\subsubsection{Conditioned Media}

To make conditioned media for experiment controls, donor cells were seeded into complete media (see 2.1.1) of the recipient cell type at 50,000 cells $/ \mathrm{mL}$ and cultured in the media for 16 hours. Upon completion cells were pelleted out of the media and the media was filtered at $0.2 \mu \mathrm{m}$ before addition onto experimental cells.

\subsubsection{Freezing Media}

Cell line freezing media was composed of either 70\% RPMI-1640 or 70\% DMEM, 20\% FBS and $10 \%$ DMSO.

\subsubsection{Flow Cytometry Buffer}

FACS Buffer used in flow cytometry experiments was composed of dPBS, 5\% FBS and $1 \mathrm{~mL}$ of EDTA. 


\subsection{Ethics}

Ethical approval for collection and manipulations of primary peripheral blood samples was given on 22 February 2018 by the University of Otago Human Ethics Committee under the reference number H17/149.

\subsection{Cell Lines}

\subsubsection{HEL}

HEL is a human erythroleukemia isolated from a patient that presented with Hodgkin's Disease 83. HEL suspension cells were cultured using complete RPMI-1640 media.

\subsubsection{K562}

K562 is a chronic myelogenous leukemia obtained from a patient in the 1970s 85 . K562 suspension cells were cultured in complete RPMI-1640 media.

\subsubsection{HL-60}

HL-60 is characterized as an acute myeloid leukemia-myeloblastic subtype 43. HL-60 suspension cells were cultured using complete DMEM media.

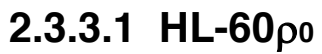

HL-60 مo cells were derived at the Malaghan Institute of Medical Research by Dr Patries Herst from parental HL-60 cells using exposure to low-dose ethidium bromide in cell culture to deplete their mitochondrial DNA 43.

HL-60 مo cells were cultured in complete DMEM media supplemented with $1 \mathrm{mM}$ sodium pyruvate and $50 \mu \mathrm{g} / \mathrm{mL}$ uridine. When depriving HL-60 po cells, different DMEM - high glucose media lacking any sodium pyruvate and uridine - supplemented with 10\% FBS and 1\% Glutamax was used. 


\subsubsection{Cell Culture}

All cell lines were obtained from the stocks of the Cancer Cell Biology Group of the Malaghan Institute of Medical Research (MIMR, Wellington). Primary cells were obtained from donors who had consented to all the relevant forms by trained phlebotomists. Cells were handled under sterile HERASafe hoods with HEPA air filters and incubated at $37^{\circ} \mathrm{C}$ and $5 \% \mathrm{CO}_{2}$ in a humidified incubator. Cell concentrations unless otherwise specified were maintained between $5 \times 10_{5}$ and $1 \times 106$ cells $/ \mathrm{mL}$ in suitable flasks. Centrifugation steps were spun at $1000 \mathrm{x} g$ for 5 minutes.

\subsubsection{Freezing Protocol}

Cell suspensions were counted using Trypan Blue Viability staining on a Hemocytometer. Cells were pelleted using the Megafuge 2.0 in Falcon tubes. Cell pellets were resuspended to $1 \times 106-4 \times 106$ cells $/ \mathrm{mL}$ in respective freezing media. $1 \mathrm{~mL}$ of freezing suspension was aliquoted into $1.5 \mathrm{~mL}$ Cryovials and place in a -80 Freezer in a Nalgene $\mathrm{TM}^{\mathrm{TM}} \mathrm{Mr}$ Frosty to cool the vials $1{ }^{\circ} \mathrm{C} / \mathrm{min}$ overnight. The following day, cells were transferred to the liquid nitrogen tanks for storage.

\subsubsection{Drug Treatments}

\subsubsection{Deferoxamine}

The drug, Deferoxamine (DFO), is stored in its powder form in a $-20^{\circ} \mathrm{C}$ freezer. To solubilise DFO for use in culture it was dissolved in sterile distilled water at $50 \mathrm{mg} / \mathrm{mL}$ fresh for each experiment. Concentrations between $10 \mu \mathrm{M}$ and $300 \mu \mathrm{M}$ were used in this thesis.

\subsubsection{Cisplatin}

Cisplatin is stored in a crystallised form in a $-20^{\circ} \mathrm{C}$ freezer. To solubilised Cisplatin for use in culture it was dissolved in sterile $0.2 \mu \mathrm{m}$ filtered $0.9 \% \mathrm{NaCl}$ solution at $1 \mathrm{mg} / \mathrm{mL}$. This solution was made by dissolving a ratio of $9 \mathrm{~g} \mathrm{NaCl}: 1 \mathrm{~L}$ sterile deionised water at the desired volume. Cisplatin solutions were stored in the dark in $4^{\circ} \mathrm{C}$ fridge no longer than 3 weeks. Concentrations between $1 \mu \mathrm{M}$ and $100 \mu \mathrm{M}$ were used in this thesis. 


\subsection{Primary Erythroblast Cells}

\subsubsection{PBMC Isolation}

Pre-determined volumes of blood were drawn by trained phlebotomists at the MIMR into $10 \mathrm{~mL}$ purple top EDTA tubes and inverted to prevent clotting. Blood and all reagents for PBMC isolation were placed into a sterile hood for the duration of the process. Blood was mixed 1:1 with room temperature sterile PBS in a $50 \mathrm{~mL}$ falcon tube before layering gently onto room temperature sterile Lymphoprep using the lowest expulsion rate and a $10 \mathrm{~mL}$ serological pipette. Tubes with the blood:lymphoprep layering were placed gently into a balanced centrifuge with bucket covers and spun at $800 \mathrm{x}$ g for 30 minutes at $18-20^{\circ} \mathrm{C}$ without the brake on.

The top layer of plasma and serum was removed using a $10 \mathrm{~mL}$ serological pipette and disposed of in bleach. To remove the layer of PBMCs - the white buffy coat $-1 \mathrm{~mL}$ sterile Pasteur pipettes were used and cells placed into $15 \mathrm{~mL}$ tubes. PBMCs were diluted in at least $10 \mathrm{~mL}$ of PBS as the first wash and spun in the centrifuge at $800 \mathrm{xg}$ for 10 minutes with the brake on. Cells were resuspended in $10 \mathrm{~mL}$ fresh PBS for a second wash step. Prior to pelleting a second time cells were counted and viability assessed using Trypan Blue and a Hemocytometer. Cells were then spun for the final time at $600 \mathrm{x}$ g for 10 minutes and resuspended in media at volumes appropriate for downstream applications.

\subsubsection{Erythroblast Base Culture Media}

Base erythroblast culture media was constructed as outlined in 87. Media consisted of IMDM with $25 \mathrm{mM}$ HEPES, 20\% FBS, 1\% Glutamax, 1\% Penicillin/Streptomycin and 7.5x10-5 M $\beta$-mercaptoethanol. It also contained $10 \mathrm{ng} / \mathrm{mL}$ human Stem Cell Factor, 3 Units/mL of human erythropoietin, $1 \mathrm{ng} / \mathrm{mL}$ of human IL-3 and $1 \mu \mathrm{M}$ human dexamethasone added once media was to be put onto cells in culture.

\subsubsection{Erythroblast Culture Method}

The method described by Migliaccio and colleagues (2010) was used to culture erythroblasts from PBMCs. PBMCs are maintained throughout the culturing process at $1 \mathrm{x} 106$ cells $/ \mathrm{mL}$. Every 48 hours from days $0-10$ extra dexamethasone is added at a 
$0.5 \% \mathrm{v} / \mathrm{v}$ concentration from a $1 \mathrm{mM}$ working stock. From day 10 onwards base media now contains all components described in 2.4.2 and $1 \mu \mathrm{M} \beta$-estradiol. Every 48 hours from this point both dexamethasone ( $1 \mu \mathrm{M}$ stock) and $\beta$-estradiol (100 $\mu \mathrm{M}$ stock) are added to culture media at $0.5 \% \mathrm{v} / \mathrm{v}$ concentration. The culture method takes approximately 17 days.

\subsection{Molecular Methods}

\subsubsection{Primer Design}

New primers were designed using sequences visualised in Geneious version 8.0.5 and analysed using the online Integrated DNA Technologies (IDT) Oligo Calculator before ordering from IDT. The location of the primer sets used for sequencing on the mitochondrial genome are shown in Figure 2.1

\subsubsection{DNA Extraction}

Two kits were used to extract genomic and mitochondrial DNA from experimental samples. Where cell number was greater than 1x106 the Zymo Research Quick gDNA $^{\mathrm{TM}}$ Miniprep Kit was used. Where cell number was less than 1x106 the Zymo Research Quick-gDNA Microprep Kit was used. The recommended protocol for both kits was used with slight modification: lysed cell samples were loaded through the column twice, the wash step was completed twice, elution buffers were heated to $60^{\circ} \mathrm{C}$ and after each centrifugation step every column was checked to ensure all solution had passed through - if not then columns were spun again. The final elutions were collected in $1.7 \mathrm{~mL}$ microfuge tubes and analysed using the Nanodrop One for concentration and purity. All extracted samples were stored at either $4{ }^{\circ} \mathrm{C}$ for same day use or at $-80^{\circ} \mathrm{C}$ for future use. 


\subsubsection{Human Mitochondrial DNA Sequencing}

Table 2.5.1 List of Primers for PCR and Sequencing

\begin{tabular}{|c|c|c|c|c|}
\hline $\begin{array}{l}\text { Primer } \\
\text { Name }\end{array}$ & Direction & Position & Sequence & Supplier \\
\hline $\begin{array}{c}\text { mt_Dloop_ } \\
\text { F }\end{array}$ & Forward & $\begin{array}{c}15.978- \\
15.997\end{array}$ & $\begin{array}{c}\text { '- } \\
\text { CACCATTAGCACCCAAA } \\
\text { GCT-3' }\end{array}$ & $\begin{array}{c}\text { Integrated } \\
\text { DNA } \\
\text { Technologies }\end{array}$ \\
\hline $\begin{array}{c}\mathrm{mt} \text { _Dloop_ } \\
\mathrm{R}\end{array}$ & Reverse & $409-429$ & $\begin{array}{c}\text { 5'- } \\
\text { CTGTTAAAAGTGCATAC } \\
\text { CGCC-3' }\end{array}$ & $\begin{array}{c}\text { Integrated } \\
\text { DNA } \\
\text { Technologies }\end{array}$ \\
\hline $\begin{array}{c}\text { mt_PolyC_ } \\
\text { F }\end{array}$ & Forward & $\begin{array}{c}16.525- \\
16.544\end{array}$ & $\begin{array}{c}\text { 5'- } \\
\text { AGCCTAAATAGCCCACA } \\
\text { CGT-3' }\end{array}$ & $\begin{array}{c}\text { Integrated } \\
\text { DNA } \\
\text { Technologies }\end{array}$ \\
\hline $\begin{array}{c}\text { mt_PolyC_ } \\
\mathrm{R}\end{array}$ & Reverse & $806-825$ & $\begin{array}{c}\text { 5'- } \\
\text { AATCACTGCTGTTTCCCG } \\
\text { TG-3' }\end{array}$ & $\begin{array}{c}\text { Integrated } \\
\text { DNA } \\
\text { Technologies }\end{array}$ \\
\hline
\end{tabular}

Obtained from Victoria University of Wellington Cell Survival Group.

\subsubsection{Cell Line Sequencing PCR}

Individual PCR reactions composed of $10 \mu \mathrm{L}$ of KAPA HiFi HotStart ReadyMix, 0.6 $\mu \mathrm{L}$ of $10 \mu \mathrm{M}$ forward primer, $0.6 \mu \mathrm{L}$ of $10 \mu \mathrm{M}$ reverse primer, approximately $20 \mathrm{ng}$ of template DNA with the final reaction volume made up to $20 \mu \mathrm{L}$ with PCR-grade nuclease free water. Both 'D-loop' and 'poly-C' products were generated for each sample. A no-template control (NTC) was included for each primer set.

The cycling protocol was as follows: preheat to $105^{\circ} \mathrm{C}$, Initial Denaturation at $95^{\circ} \mathrm{C}$ for 3 minutes, 30 cycles of denaturation at $95^{\circ} \mathrm{C}$ for 20 seconds, annealing at $65^{\circ} \mathrm{C}$ (for Dloop) or $66^{\circ} \mathrm{C}$ (for PolyC) for 15 seconds and extension at $72^{\circ} \mathrm{C}$ for 30 seconds, with a final extension at $72^{\circ} \mathrm{C}$ for 3 minutes. Upon conclusion, PCR reactions were stored in a fridge at $4^{\circ} \mathrm{C}$ until use in gel electrophoresis and sequencing reactions. 


\subsubsection{Whole Blood Primary Sequencing PCR}

To sequence potential donors for mitochondrial DNA polymorphisms suitable for coculture experiments, direct PCR on whole blood was conducted. To collect blood, donors were instructed to clean their ring finger with 70\% ethanol and use a Unistik® 3 Normal Lancet to draw blood. Blood was squeezed into a $0.2 \mathrm{~mL}$ microfuge tube after wiping away the first few drops.

$30 \mu \mathrm{L}$ PCR reactions were composed of $15 \mu \mathrm{L}$ of Invitrogen ${ }^{\mathrm{TM}}$ Platinum $^{\mathrm{TM}}$ SuperFi ${ }^{\mathrm{TM}}$ PCR Master Mix, $1.2 \mu \mathrm{L}$ of $10 \mu \mathrm{M}$ forward primer, $1.2 \mu \mathrm{L}$ of reverse primer, $2 \mu \mathrm{L}$ of blood and 10.6 $\mu \mathrm{L}$ of PCR grade water. Both "D-loop" and "poly-C" products were generated for each sample. For each product and set of reactions an NTC with water template was used as a negative control and a sample of cell line DNA was used as a positive control.

The final cycling protocol was as follows: preheat to $105^{\circ} \mathrm{C}$, Initial Denaturation at $98^{\circ} \mathrm{C}$ for 5 minutes, 30 cycles of denaturation at $98^{\circ} \mathrm{C}$ for 10 seconds, annealing at $63^{\circ} \mathrm{C}$ (for D-loop product) or $66^{\circ} \mathrm{C}$ (for Poly_C product) for 10 seconds and extension at $72^{\circ} \mathrm{C}$ for 30 seconds, and a final extension at $72^{\circ} \mathrm{C}$ for 5 minutes. Upon conclusion, PCR reactions were stored in a fridge at $4^{\circ} \mathrm{C}$ until use in gel electrophoresis and sequencing reactions.

\subsubsection{Gel Electrophoresis}

PCR samples were run through 2\% Agarose Gels in a BioRad gel tank submerged in TAE Buffer. HyAgarose ${ }^{\mathrm{TM}}$ powder was dissolved in TAE by heating the solution (by microwave) in a conical flask in 2-3-minute intervals to prevent boiling over. Once the solution was clear and free of any agarose clumps or strings, it was slowly poured into a gel mold with a 15 well comb and left to set for around 30 minutes at room temperature. Any bubbles present were dispersed or popped using a pipette tip.

$4 \mu \mathrm{L}$ of DNA Ladder was added to each Gel Electrophoresis run as a control. $1 \mu \mathrm{L}$ of loading buffer and $3 \mu \mathrm{L}$ of sample were mixed on parafilm for each sample and loaded into the relevant well. Gels were run at $140 \mathrm{~V}$ until the dye had progressed approximately $75-80 \%$ down the gel. ChemiDoc ${ }^{\mathrm{TM}}$ Touch Imaging System was used to 
image the gels, which were analysed to assess the correct product length, amount and purity (against NTC). Images of the gels were edited using Image Lab version 6.

PCR products to be sequenced were purified and concentrated using the DNA Clean \& Concentrator ${ }^{\mathrm{TM}}$ Kit purchased from Zymo Research as per the manufacturer's instructions. Centrifugation steps were carried out at $14000 \mathrm{x}$ g. Concentrated samples were analysed using the NanoDrop One and either used immediately for sequencing or stored at $-4^{\circ} \mathrm{C}$ short term.

\subsubsection{Sequencing}

Sequencing reactions were conducted at Massey Genome Services in Palmerston North and were couriered overnight using NZ Courier Post. Reactions were composed in $0.2 \mathrm{~mL}$ microfuge tubes as per the recommendations of Massey Genome Services. Each PCR sample required two sequencing reactions, one for forward and one for reverse primers as applicable. To each reaction $4 \mu \mathrm{L}$ of $1 \mu \mathrm{M}$ primer stock was added giving a final concentration of 4 pmol, approximately $25 \mathrm{ng}$ of template was added, and DEPCtreated nuclease free water was added to the final volume of $20 \mu \mathrm{L}$.

\subsubsection{Analysis}

Sequences were obtained electronically from the Massey Genomes Services website and analysed on the computer program Geneious version 8.0.5. Sequences were aligned to each other and a reference sequence NC_012920.1 obtained from NCBI. 


\subsubsection{Mitochondrial Copy Number Endpoint PCR}

Table 2.5.2 Nuclear Control PCR Primers

\begin{tabular}{|c|c|c|c|}
\hline $\begin{array}{c}\text { Primer } \\
\text { Name }\end{array}$ & Position & Sequence & Supplier \\
\hline hu_Beta- & $\begin{array}{c}\text { Chr } 11 \\
5,226,949-\end{array}$ & 5 '- \\
Globin-F & $5,226,969$ & CAACTTCATCCACGTTCACC-3' & $\begin{array}{c}\text { Integrated DNA } \\
\text { Technologies }\end{array}$ \\
\hline hu_Beta- & Chr 11 & 5 '- & Integrated DNA \\
Globin-R & $5,227,197-$ & GAAGAGCCAAGGACAGTTAC- & Technologies \\
\hline
\end{tabular}

Primers previously published 19.

\subsubsection{PCR Protocol}

Two separate reactions were run; one for Dloop sequencing primers as in Table 2.5.1 and one as a nuclear control using $\beta$-globin, both on the same sample of DNA. An NTC and a sample of parental cell DNA as a positive control were included. $10 \mu \mathrm{L}$ reactions were composed of $0.4 \mu \mathrm{L}$ of each primer, $5 \mu \mathrm{L}$ of Invitrogen ${ }^{\mathrm{TM}}$ Platinum $^{\mathrm{TM}}$ SuperFi $^{\mathrm{TM}}$ PCR Master Mix, $2.2 \mu \mathrm{L}$ Nuclease Free water and $2 \mu \mathrm{L}$ of template DNA. PCR cycling protocol was as described in 2.5.3.2 using the $63^{\circ} \mathrm{C}$ annealing temperature for the Dloop product and $54^{\circ} \mathrm{C}$ annealing temperature for the nuclear products. Products were run out on a $\%$ Agarose gel, imaged and edited as described in 2.5.3.3. 
2.5.5 Allele-Specific-Blocker qPCR

Table 2.5.3 SNP195 ASBqPCR mtDNA Primer Set

\begin{tabular}{|c|c|c|c|}
\hline Primer Name & Position & Sequence & Supplier \\
\hline $\begin{array}{c}\text { ASBsnp88U87 } \\
\text { Fwd }\end{array}$ & $241-259$ & 5'-GTGCAGACATTCAATTGT-3' & $\begin{array}{c}\text { Integrated } \\
\text { DNA } \\
\text { Technologies }\end{array}$ \\
\hline ASsnp88Rev & $181-195$ & 5'-ACAGGCGAACATACC-3' & $\begin{array}{c}\text { Integrated } \\
\text { DNA } \\
\text { Technologies }\end{array}$ \\
\hline $\begin{array}{c}\text { BN18snp88Rev } \\
\text { Blocker }\end{array}$ & $186-208$ & 5'-CGAACATACTTACTAAAGTGTG & $\begin{array}{c}\text { Integrated } \\
\text { DNA } \\
\text { ASBsnp88 }\end{array}$ \\
$\begin{array}{c}\text { Hydrolysis } \\
\text { Probe }\end{array}$ & $204-234$ & /3Phos/-3' & Technologies \\
\hline
\end{tabular}

Designed and obtained from Victoria University of Wellington Cell Survival Group

Table 2.5.4 SNP150/152 ASBqPCR Primer Set

\begin{tabular}{|c|c|c|c|}
\hline Primer Name & Position & Sequence & Supplier \\
$\begin{array}{c}\text { ASsnp150/152 } \\
\text { TC HL60 }\end{array}$ & $137-152$ & 5'-ATTCCTGCCTCATTCC-3' & $\begin{array}{c}\text { Integrated } \\
\text { DNA } \\
\text { Technologies }\end{array}$ \\
\hline $\begin{array}{c}\text { ASBsnp150/15 } \\
\text { 2 Blocker }\end{array}$ & $146-167$ & TCATCCTATTATTTATCGCACCT & $\begin{array}{c}\text { Integrated } \\
\text { DNA } \\
\text { ASBsnp88U87 } \\
\text { Fwd }\end{array}$ \\
\hline $\begin{array}{c}\text { ASBsnp88 } \\
\text { Hydrolysis } \\
\text { Probe }\end{array}$ & $241-259$ & 5'-GTGCAGACATTCAATTGT-3' & $\begin{array}{c}\text { Integrated } \\
\text { DNA }\end{array}$ \\
\hline
\end{tabular}


Table 2.5.5 hu_MPB09 ASBqPCR Primer Set

\begin{tabular}{|c|c|c|c|}
\hline Primer Name & Position & Sequence & Supplier \\
\hline hu_ASsnp152_C_09 & $137-152$ & 5'- ATTCCTGCCTCATCCC -3' & $\begin{array}{c}\text { Integrated } \\
\text { DNA } \\
\text { Technologies }\end{array}$ \\
\hline $\begin{array}{c}\text { LN18snp88RevBloc } \\
\text { ker }\end{array}$ & $186-208$ & $\begin{array}{c}5^{\prime}- \\
\text { CGAACATACTTACTAAAGT } \\
\text { GTG/ 3Phos/-3' }\end{array}$ & $\begin{array}{c}\text { Integrated } \\
\text { DNA } \\
\text { Technologies }\end{array}$ \\
\hline ASBsnp88U87 Fwd & $241-259$ & $\begin{array}{c}5 '- \\
\text { GTGCAGACATTCAATTGT-3' }\end{array}$ & $\begin{array}{c}\text { Integrated } \\
\text { DNA } \\
\text { Technologies }\end{array}$ \\
\hline $\begin{array}{l}\text { hu_ASB } \\
\text { SNP152_C_09 } \\
\text { Hydrolysis Probe }\end{array}$ & 204-234 & $\begin{array}{c}\text { 5'-/56- } \\
\text { FAM/TGTGTTAAT/ZEN/ } \\
\text { TAATCAATGCTTGTAGGAC } \\
\text { AT/ } \\
\text { 3IABkFQ/ -3' }\end{array}$ & $\begin{array}{c}\text { Integrated } \\
\text { DNA } \\
\text { Technologies }\end{array}$ \\
\hline
\end{tabular}

\subsubsection{Annealing Gradients}

End-point PCR was conducted using the primer sets described in Tables 2.5.3-2.5.5 above excluding the fluorescent hydrolysis probe. An NTC control was included in the analysis and samples were tested at a range of temperatures between $50-60^{\circ} \mathrm{C}$. The reaction was run on both on and off target template at each temperature. Additionally, at the predicted temperature, on and off target template reactions were run without the blocker primer present.

For the SNP195 ASBqPCR assay, $10 \mu \mathrm{L}$ reactions consisted of $0.2 \mu \mathrm{L}$ of a $10 \mu \mathrm{M}$ stock for the forward and reverse primers, $1.2 \mu \mathrm{L}$ of $10 \mu \mathrm{M}$ LN18snp88RevBlocker Primer stock, $2 \mu \mathrm{L}$ of $5 \mathrm{x}$ HOT FIREPol ${ }^{\circledR}$ Probe Universal qPCR Master Mix, $5.4 \mu \mathrm{L}$ of nuclease free water and $1 \mu \mathrm{L}$ of template for the corresponding tube. The cycling conditions were $95^{\circ} \mathrm{C} 12$-minute HotStart followed by 35 cycles of denaturation at $95^{\circ} \mathrm{C}$ for 15 seconds, annealing temperature gradient from $52-60.6^{\circ} \mathrm{C}$ for 30 seconds and 
extension at $60^{\circ} \mathrm{C}$ for 30 seconds, followed by a final 3 -minute extension at $60^{\circ} \mathrm{C}$. Samples were analysed by electrophoresis as per 2.5.3.3.

For the SNP150/152 ASBqPCR assay, $10 \mu \mathrm{L}$ reactions consisted of $0.4 \mu \mathrm{L}$ of a $10 \mu \mathrm{M}$ stock for the forward and reverse primers, $1.6 \mu \mathrm{L}$ of a $10 \mu \mathrm{M}$ ASBsnp150/152Blocker Primer stock, $2 \mu \mathrm{L}$ of $5 \mathrm{x}$ HOT FIREPol Probe Universal qPCR Master Mix, $4.6 \mu \mathrm{L}$ of nuclease free water and $1 \mu \mathrm{L}$ template for the corresponding tube. The cycling conditions were $95^{\circ} \mathrm{C} 12$-minute HotStart followed by 35 cycles of denaturation at $95^{\circ} \mathrm{C}$ for 15 seconds, annealing temperature gradient from $50-60^{\circ} \mathrm{C}$ for 30 seconds and extension at $60^{\circ} \mathrm{C}$ for 30 seconds, followed by a final 3 -minute extension at $60^{\circ} \mathrm{C}$. Samples were analysed by electrophoresis as per 2.5.3.3.

For the hu_MPB09 ASBqPCR assay, $10 \mu \mathrm{L}$ reactions consisted of $0.4 \mu \mathrm{L}$ of a $10 \mu \mathrm{M}$ stock for the forward and reverse primers, $1.6 \mu \mathrm{L}$ of a $10 \mu \mathrm{M}$ LN18snp88RevBlocker Primer stock, $2 \mu \mathrm{L}$ of $5 \mathrm{x}$ HOT FIREPol ${ }^{\circledR}$ Probe Universal qPCR Master Mix, $3.6 \mu \mathrm{L}$ of nuclease free water and $2 \mu \mathrm{L}$ template for the corresponding tube. The cycling conditions were $95^{\circ} \mathrm{C} 12$-minute HotStart followed by 35 cycles of denaturation at $95^{\circ} \mathrm{C}$ for 15 seconds, annealing temperature gradient from $50-65^{\circ} \mathrm{C}$ for 30 seconds and extension at $60^{\circ} \mathrm{C}$ for 30 seconds, followed by a final 3 -minute extension at $60^{\circ} \mathrm{C}$. Samples were analysed by electrophoresis as per 2.5.3.3.

\subsubsection{ASBqPCR Controls}

At least 3 replicate NTC wells were run on all experimental plates. All DNA samples were used at $20 \mathrm{ng} / \mathrm{well}$. Use of 96-well or 384-well plates were experiment-dependent.

To generate a standard curve, a proportion of stock on-target gDNA was serially diluted $1: 10$ into 5 tubes to make 6 dilutions ranging from $100 \%$ to $0.001 \%(1: 100,000)$. Triplicate wells of each dilution were run on each plate to simulate the standard curve. Triplicate wells of a $100 \%$ stock off-target gDNA template were also run to show the limit of detection for each assay run.

To simulate transfer of on-target mtDNA into an off-target gDNA and mtDNA background, an artificial spike-in standard curve was generated for every plate and run in triplicate wells for each sample. A sample of the on-target serial dilutions made as 
part of the standard curve were mixed 1:1 with 100\% off-target gDNA template (excluding 100\% on-target sample).

2.5.5.3 SNP195 ASBqPCR Protocol

\begin{tabular}{|c|c|c|c|}
\hline Component & $\begin{array}{c}\text { Stock } \\
\text { Concentration }\end{array}$ & $\begin{array}{c}\text { Final } \\
\text { Concentration }\end{array}$ & $\begin{array}{c}\text { Volume in } \\
\text { Single Reaction } \\
(\mu \mathbf{L})\end{array}$ \\
\hline ASBsnp88U87 Fwd & $10 \mu \mathrm{M}$ & $0.2 \mu \mathrm{M}$ & 0.2 \\
\hline ASsnp88Rev & $10 \mu \mathrm{M}$ & $0.2 \mu \mathrm{M}$ & 0.2 \\
\hline $\begin{array}{c}\text { LN18snp88RevBlocker } \\
\text { Universal qPCR Master } \\
\text { Mix }\end{array}$ & $10 \mu \mathrm{M}$ & $1.2 \mu \mathrm{M}$ & 1.2 \\
\hline $\begin{array}{c}\text { ASBsnp88 Hydrolysis } \\
\text { Probe }\end{array}$ & $1 \mu \mathrm{M}$ & $0.2 \mu \mathrm{M}$ & 2 \\
\hline PCR-Grade Water & $\mathrm{N} / \mathrm{A}$ & $\mathrm{N} / \mathrm{A}$ & 2.4 \\
\hline Template & $10 \mathrm{ng} / \mu \mathrm{L}$ & $20 \mathrm{ng} / \mathrm{well}$ & 2 \\
\hline \multicolumn{2}{|c|}{} & Total & 10 \\
\hline
\end{tabular}

\begin{tabular}{|c|c|c|c|}
\hline Cycling Step & Time & Temperature $\left({ }^{\circ} \mathbf{C}\right)$ & Number of Cycles \\
\hline Hot Start & 12 minutes & 95 & 1 \\
\hline Denaturation & 15 seconds & 95 & \multirow{2}{*}{45} \\
\hline Annealing & 30 seconds & 53 & \\
\hline Extension & 30 seconds & 60 & \\
\hline
\end{tabular}


2.5.5.4 SNP150/152 ASBqPCR Protocol

\begin{tabular}{|c|c|c|c|}
\hline Component & $\begin{array}{c}\text { Stock } \\
\text { Concentration }\end{array}$ & $\begin{array}{c}\text { Final } \\
\text { Concentration }\end{array}$ & $\begin{array}{c}\text { Volume in Single } \\
\text { Reaction }(\mu \mathbf{L})\end{array}$ \\
\hline ASsnp150/152TC HL60 & $10 \mu \mathrm{M}$ & $0.4 \mu \mathrm{M}$ & 0.4 \\
\hline ASBsnp88U87 Fwd & $10 \mu \mathrm{M}$ & $0.4 \mu \mathrm{M}$ & 1.6 \\
\hline $\begin{array}{c}\text { ASBsnp150/152 } \\
\text { Blocker }\end{array}$ & $10 \mu \mathrm{M}$ & $1.6 \mu \mathrm{M}$ & 2 \\
\hline $\begin{array}{c}\text { HOT FIREPol }{ }^{\circledR} \text { Probe } \\
\text { Universal qPCR Master } \\
\text { Mix }\end{array}$ & $5 \mathrm{x}$ & $1 \mathrm{x}$ & \\
\hline ASBsnp88 Hydrolysis \\
Probe
\end{tabular}

\begin{tabular}{|c|c|c|c|}
\hline Cycling Step & Time & Temperature $\left({ }^{\circ} \mathbf{C}\right)$ & Number of Cycles \\
\hline Hot Start & 12 minutes & 95 & 1 \\
\hline Denaturation & 15 seconds & 95 & \multirow{2}{*}{45} \\
\hline Annealing & 30 seconds & 54 & \\
\hline Extension & 30 seconds & 60 & \\
\hline
\end{tabular}


2.5.5.5 hu_MPB09 ASBqPCR Protocol

\begin{tabular}{|c|c|c|c|}
\hline Component & $\begin{array}{c}\text { Stock } \\
\text { Concentration }\end{array}$ & $\begin{array}{c}\text { Final } \\
\text { Concentration }\end{array}$ & $\begin{array}{c}\text { Volume in } \\
\text { Single Reaction } \\
(\mu \mathbf{L})\end{array}$ \\
\hline hu_ASsnp152_C_09 & $10 \mu \mathrm{M}$ & $0.4 \mu \mathrm{M}$ & 0.4 \\
\hline ASBsnp88U87 Fwd & $10 \mu \mathrm{M}$ & $0.4 \mu \mathrm{M}$ & 0.4 \\
\hline LN18snp88RevBlocker & $10 \mu \mathrm{M}$ & $1.6 \mu \mathrm{M}$ & 1.6 \\
\hline $\begin{array}{c}\text { HOT FIREPol@ Probe } \\
\text { Universal qPCR Master } \\
\text { Mix }\end{array}$ & $5 \mathrm{x}$ & $1 \mathrm{x}$ & 2 \\
\hline hu_ASB SNP152_C_09 & $1 \mu \mathrm{M}$ & $0.2 \mu \mathrm{M}$ & 2 \\
\hline Hydrolysis Probe & N/A & N/A & 1.6 \\
\hline PCR-Grade Water & $10 \mathrm{ng} / \mu \mathrm{L}$ & $20 \mathrm{ng} / \mathrm{well}$ & 2 \\
\hline Template & & Total & 10 \\
\hline
\end{tabular}

\begin{tabular}{|c|c|c|c|}
\hline Cycling Step & Time & Temperature $\left({ }^{\circ} \mathbf{C}\right)$ & Number of Cycles \\
\hline Hot Start & 12 minutes & 95 & 1 \\
\hline Denaturation & 15 seconds & 95 & \multirow{2}{*}{45} \\
\hline Annealing & 30 seconds & 56 & \\
\hline Extension & 30 seconds & 60 & \\
\hline
\end{tabular}

\subsubsection{ASBqPCR analysis}

Data obtained from qPCR reactions were recorded and analysed using the Quantstudio $^{\text {TM }}$ Real Time PCR Software v1.3. Additional manipulations were carried out in Prism 8 and Microsoft Excel. 


\subsection{Flow Cytometry}

Table 2.6.1 List of Cell Surface Antibodies

\begin{tabular}{|c|c|c|c|c|}
\hline Antigen & Fluorophore & Manufacturer & Clone & Catalog \# \\
\hline CD117 & Alexa Fluor 647 & Biolegend & $104 \mathrm{D} 2$ & 313235 \\
\hline CD235a & $\begin{array}{c}\text { Phycoerythrin } \\
(\mathrm{PE})\end{array}$ & $\begin{array}{c}\text { Beckman } \\
\text { Coulter }\end{array}$ & $\begin{array}{c}11 \mathrm{E} 4 \mathrm{~B}-7-6 \\
(\mathrm{KC} 16)\end{array}$ & IM2211U \\
\hline CD71 & $\begin{array}{c}\text { Fluorescein } \\
\text { isothiocyanate } \\
\text { (FITC) }\end{array}$ & BD Biosciences & L01.1 & 347513 \\
\hline CD38 & $\begin{array}{c}\text { Allophycocyanin } \\
\text { (APC) }\end{array}$ & BD Biosciences & HB7 & 340439 \\
\hline
\end{tabular}

Table 2.6.2 List of Fluorescent Dyes and Proteins

\begin{tabular}{|c|c|c|}
\hline Dye/Protein & Manufacturer & Catalog \# \\
\hline Zombie NIR $^{\mathrm{TM}}$ & Biolegend & 423106 \\
\hline MitoView $^{\mathrm{TM}}$ Blue & Biotium & 70070 \\
\hline Viafluor $^{\circledR} 405-\mathrm{SE}$ & Biotium & BIT30068 \\
\hline Cell Trace $^{\mathrm{TM}}$ Far Red & Invitrogen $^{\mathrm{TM}}$ & $\mathrm{C} 345629$ \\
\hline Cell Trace $^{\mathrm{TM}}$ CFSE & Invitrogen $^{\mathrm{TM}}$ & $\mathrm{C} 34554$ \\
\hline Annexin V APC & Biolegend $^{6}$ & 640941 \\
\hline
\end{tabular}

\subsubsection{Viability Dyes}

\subsubsection{DAPI}

DAPI was diluted 1:5000 in sterile dPBS and stored in the dark, wrapped in foil, at $4^{\circ} \mathrm{C}$.

\subsubsection{Propidium lodide}

Propidium iodide was stored at $0.5 \mathrm{mg} / \mathrm{mL}$ in water wrapped in foil at $4^{\circ} \mathrm{C}$. 


\subsubsection{Flow Cytometry Controls}

For each experiment, cytometry controls consisted of unstained controls, single stains on either UltraComp ebeads ${ }^{\mathrm{TM}}$, or cells and 'fluorescence minus one' controls on panels of three colours or more.

\subsubsection{Flow Cytometry Protocol}

Experiment dependent parameters were determined on an individual basis. These parameters include cell numbers, antibody combinations and volumes for staining and running samples on the cytometers. Individual antibody and viability concentrations were pre-determined using titrations.

\subsubsection{Surface Antibodies}

Desired numbers of cells were counted using Trypan Blue and a hemocytometer.

Required volumes of cells were placed into appropriate collection tubes and centrifuged at $1000 \mathrm{x}$ g for 5 minutes. Cells were resuspended in FACS Buffer to $1 \times 106$ cells per $100 \mu \mathrm{L}$ and aliquoted into $1.7 \mathrm{~mL}$ microfuge tubes. After pelleting cells a second time, pre-determined buffer volume and antibodies were added to each sample. Where beads were used for compensation controls, 1-2 drops of pre-vortexed beads were added into the relevant tubes then treated as cells. Samples were incubated in the dark at room temperature (approx. $25 \mathrm{oC}$ ) for 15-20 minutes. To wash off the unbound antibodies, samples were washed twice by pelleting cells and replacing the FACS buffer. $200 \mu \mathrm{L}$ of FACS buffer was used for each wash. Final resuspension volume of cells was determined per experiment to allow for addition of viability stains. At least $500 \mu \mathrm{L}$ of filtered suspension per sample was in each Falcon ${ }^{\mathrm{TM}}$ Polystyrene Round Bottom Tube. Where primary cells were used, the tubes would be capped.

\subsubsection{Apoptosis Panel}

Cells were prepared and aliquoted as per 2.6.3.1 into their samples. A master mix of Zombie NIR - at a final 1:1250 dilution - and Mitoview Blue - at $200 \mu \mathrm{M}$ final concentration - was made using $1 \mathrm{X}$ Annexin Binding Buffer and added to respective samples. Annexin V APC was then added and all samples were incubated for 15 minutes at room temperature in the dark. Cells were then washed twice as above 2.6.3.1 
and reconstituted using 1x Annexin Binding buffer in Falcon ${ }^{\mathrm{TM}}$ Polystyrene Round Bottom Tubes to be run on the cytometers.

\subsubsection{Cell Proliferation Dyes}

Cells were manipulated as described in 2.6.3.2 to be stained as per manufacturer's instructions for using Cell Trace ${ }^{\mathrm{TM}}$ and Viafluor ${ }^{\circledR}$ dyes. Briefly, cells were resuspended in pre-warmed dPBS at a concentration of $1 \times 106$ cells $/ \mathrm{mL}$ in 15 or $50 \mathrm{~mL}$ Falcon tubes. A pre-determined volume of dye reconstituted in DMSO was pipetted onto the inside of each Falcon tube lid and carefully screwed back on the tubes. Tubes were vortexed upside down for 5 seconds before being incubated at $37^{\circ} \mathrm{C}$ for 20 minutes. A volume of pre-warmed media $5 \mathrm{x}$ volume of dPBS was added to the tubes and incubated for a further 5 minutes before centrifuging $1000 \mathrm{x}$ g for 5 minutes to remove staining media. Cells were resuspended in appropriate media and flasks for further culture or FACS buffer in appropriate tubes for cytometry analysis.

Cells that were needed for cytometry analysis, were prepared through cell counts and the required volumes were pelleted. Media was aspirated off and cells were washed once with FACS buffer. Cells were resuspended in 300-500 $\mu \mathrm{L}$ in appropriate tubes and finally DAPI or PI was added prior to running samples on the cytometers.

\subsubsection{Cell Sorting Protocol}

For the purpose of sorting cells using flow cytometry, stained cells as described in 2.6.3.3 were passed through a filter cap from Falcon ${ }^{\mathrm{TM}} 5 \mathrm{~mL}$ Polystyrene Round Bottom Tubes into Falcon ${ }^{\mathrm{TM}}$ Polypropylene Round Bottom Tubes. Cells were at the required concentration of less than $10 \times 106$ cells $/ \mathrm{mL}$ for these sorts.

\subsubsection{Flow Cytometers}

Two flow cytometers were used to collect experimental data, the BD LSRII SORP (Becton Dickinson, San Jose, CA) running FACSDiva Software and Cytek Aurora (Cytek, San Francisco, CA) running SpectroFlo Software.

BD Influx ${ }^{\mathrm{TM}}$ Cell Sorter (Becton Dickinson, San Jose, CA) was used to sort cells where required. The machine was run by members of the Hugh Green Cytometry Core at MIMR. 


\subsubsection{FlowJo Analysis}

Sample data collected on the various cytometers, were all analysed using FlowJo V10 Software. A representative example of gating strategies is shown in Figure 2.2. This strategy was used unless otherwise stated in the results.

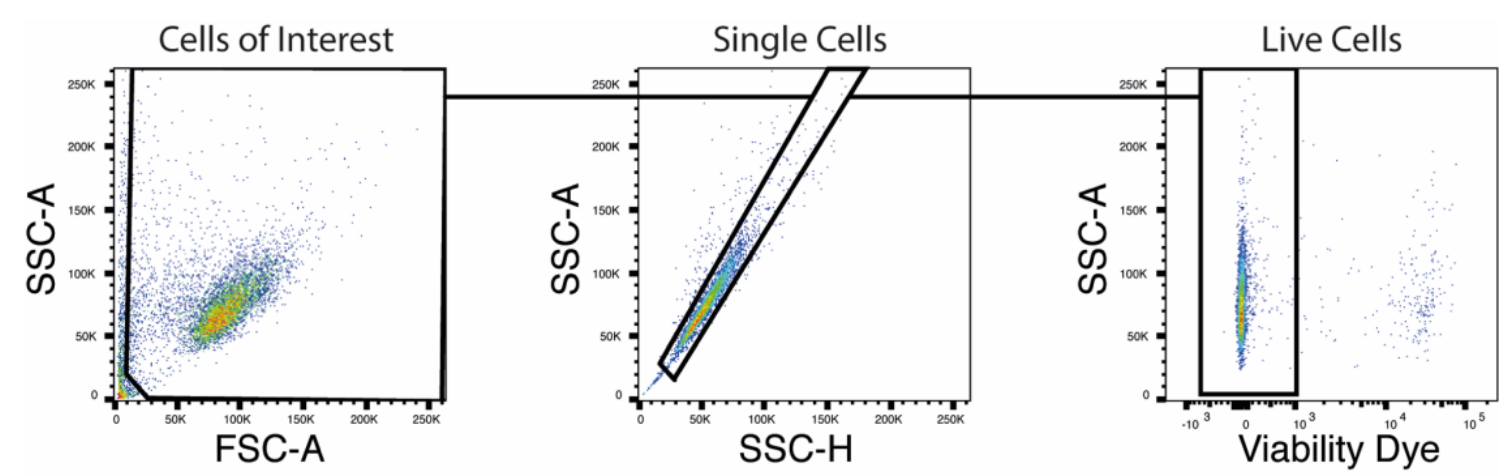

\section{Figure 2.1 Representative Gating Strategy}

Gating strategy prior to analysis unless otherwise shown in results. Cells of Interest were isolated first, drilling down to isolate single cells and then assessing viability of these cells using a viability dye such as DAPI or PI.

\subsection{Microscopy}

\subsubsection{Cell Culture Imaging}

Brightfield images of cells were taken on an Olympus IX51 Inverted Microscope (Olympus, Japan) with the following specifications: CCD Camera DP73 (Olympus, Japan), Power supply U-RFL-T (Olympus, Japan), Software Cell Sens (Olympus Soft Imaging Solutions GmbH, Münster, Germany). Images were saved as JPEGs.

\subsubsection{Perl's Stain}

Samples of cell lines and primary erythroblasts were collected into $1.7 \mathrm{~mL}$ microcentrifuge tubes, pelleted and resuspended in PBS at a concentration of 1x106 cells /mL. Cytospin funnels and SuperFrost ${ }^{\circledR}$ slides were assembled and $200 \mu \mathrm{L}$ of each sample was loaded into the corresponding funnel and placed in the Shandon Cytospin 4 to be spun at $400 \mathrm{x}$ g for 5 minutes. Once completed, slides were removed and allowed to dry. 
Slides were taken to Wellington Regional Hospital to the Wellington SCL Lab where employees completed the staining process for Perl's Stain.

\subsubsection{Protocol}

Experimental slides plus a control slide are fixed in a formalin vapour jar at $37^{\circ} \mathrm{C}$ for 30 minutes in a water bath. Once fixed, slides are placed in a coplin jar containing freshly mixed stain in a $37^{\circ} \mathrm{C}$ water bath for 30 minutes. The stain consists of equal volumes of 2\% Potassium ferrocyanide solution and 2\% HCL solution in distilled water. Slides were then rinsed with distilled water for 5 minutes and counterstained in Saffranin-O (Merck, Cat \#AB1190) by diluting $0.5 \mathrm{~mL}$ of a $0.5 \%$ solution in $50 \mathrm{~mL}$ deionised water for 20-25 minutes. Once completed, slides were very gently rinsed under a tap and allowed to air dry.

At this stage slides were returned to MIMR where DPX mounting media was added using a $1 \mathrm{~mL}$ Pasteur pipette. Coverslips were placed over mounting media and allowed to dry.

The Wellington Hospital SCL Lab also provided an image of the control slide at 20X on their Olympus BX45 microscope showing a positive result, where cells and extracellular matrix stain various intensities of red and iron clusters stain blue.

\subsubsection{Analysis}

Images of these slides were taken using the Olympus BX51TF Compound Microscope (Olympus, Japan) with the following specifications: CCD Camera DP73 (Olympus, Japan), Power Supply X-Cite 120Q (EXFO Photonic solutions Inc.), Software Cell Sens Dimension (Olympus Soft Imaging Solutions GmbH, Münster, Germany). Image were saved as JPEGs.

Slides were assessed using the microscope and representative images were taken to depict positive or negative stains when compared to the control image.

\subsubsection{Erythroblast Morphology}

Primary erythroblast cultures were cytospun as described 2.7.2. Slides with cells taken from days 8, 13 and 17 of culture were stained using Kwik-Diff protocol and reagents. 10 seconds in the fixative provided, 20 seconds in Eosin, 14 seconds in Methylene Blue, 
and 10 -seconds gentle rinsing in tap water. Slide were then left to air dry before imaging.

\subsubsection{Analysis}

Representative images were captured using the microscope described in 2.7.2.2. Population proportions of erythroblasts, lymphocytes and macrophages were identified and counted. Counting was completed by drawing $4 \times 4$ grids over images using Adobe Illustrator 2018 and cells were counted in each of the top 4 quadrants individually and a collective count of the second row. All cell populations were totalled for each image and expressed as a percentage of total cells counted to show proportions of erythroblasts, lymphocytes and macrophages. Cell characterisation was confirmed with Dr Robert Weinkove, a haematologist at Wellington Hospital and the MIMR.

\subsection{Statistical Analysis}

Statistical analysis was performed using Prism 8 software. Figure legends provide specific tests for each experiment.

Statistical tests used throughout this thesis were One-Way ANOVA, Two-Way ANOVA, and nested t-tests. Multiple comparison corrections were applied when necessary in the form of Tukey's Post hoc test for comparing every mean. P-values less than 0.05 were consider statistically significant. 
3 Exploration of mitochondrial

transfer in cells lacking

mitochondrial DNA 


\subsection{Introduction}

Mitochondria provide energy and biosynthetic molecules to allow the cell to live and divide using several proteins which are encoded by their own circular genome92. While many mitochondrial proteins are encoded by the nucleus, the mitochondrial genome contains genes for 13 important proteins involved in the tri-carboxylic acid (TCA) cycle and the electron transport chain (ETC)9. Although mitochondria hold the largest contribution to the ATP energy pool within eukaryotic cells and are involved in many other cellular processes 1 , it is possible for cells to still survive without mitochondrial genomes given the right supplements 40 .

Cells that lack mitochondrial DNA (mtDNA) are called rho zero cells ( $\rho$ o) 39,42,64,93,94. There are two ways they can be generated in lab environments, exposure to low dose ethidium bromide in cell culture or by using a restriction endonuclease targeted to the mitochondrial genome 40,43. Low dose ethidium bromide is the most common method to remove mtDNA from a cell. Ethidium bromide molecules enter into cells and intercalate with double-stranded DNA and mtDNA, not having the protection of histones, is sterically prevented from replicating and diluted out with each subsequent division in culture 40,44 . The mitochondrially targeted restriction endonuclease, termed mUNG, recognises an EcoR1 restriction site present 3-5 times in mtDNA to cleave up the whole mitochondrial genome and degrade it rapidly 40 . Ethidium bromide is not solely mitochondrially targeted like the mUNG method, therefore nuclear mutagenesis is a large limitation of the process 40 . Both of these methods result in loss of any mitochondrial genome present in cells which drastically effects their metabolism40,43. po cells lack several proteins critical for mitochondrial metabolism resulting in inability to make ATP via aerobic respiration, and synthesise pyrimidines via dihydroorotate dehydrogenase (DHODH) due to a fully reduced Complex II40. As a result, they become heavily glycolytic 39 and require supplementation of pyruvate and uridine in their environment to survive 40. Pyruvate is used to made ATP by conversion to lactate and uridine is used to synthesise pyrimidines 40 . The unique phenotype and genotype make po cells useful for exploration of whole mitochondrial transfer - organelle and genome together.

Transfer of mitochondria and mtDNA to po cells was first shown in 2006 into human

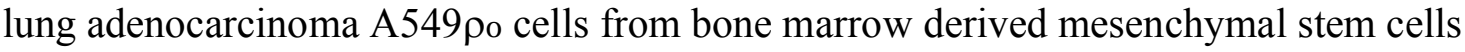


and skin fibroblasts in vitro42. Since then, transfer has been shown in other contexts

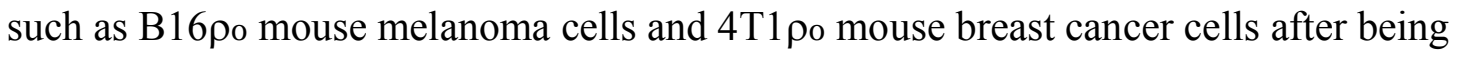
injected into mice39,64. These results suggest it is feasible, under the right conditions, to detect transfer of mitochondria into other po cell types in vitro.

This thesis has a particular focus on mitochondrial transfer between erythroblasts in the bone marrow. Several years ago, an HL-60 po line was created at the MIMR using low dose ethidium bromide exposure in cell culture from parental HL-60 cells 43. These cells are classified as acute myeloid leukemia cells 43 . To investigate if mitochondria can

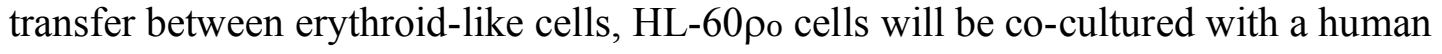
erythroleukemia cell line, HEL. This will be the first time an erythroid po cell has been studied in this context.

\subsection{Aims}

1. Characterise the cellular and molecular phenotype of HL-60 مo cells both in healthy and deprived states.

2. Assess both viability and proliferative capacity of HL-60 مo cells when healthy or deprived of uridine and pyruvate.

3. Investigate the effect of physical co-culture with a donor cell or conditioned media on viability and proliferative capacity of uridine and pyruvate deprived

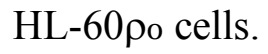

4. Assess, using a molecular Allele-Specific-Blocker qPCR assay, the presence or absence of transferred mitochondria after co-culture.

\subsection{Results}




\subsubsection{HL-60 po cells lacked detectable mitochondrial DNA and had reduced viability and proliferative capacity when deprived of uridine and pyruvate.}

po cells are classified based on their lack of mtDNA and their reliance of uridine and

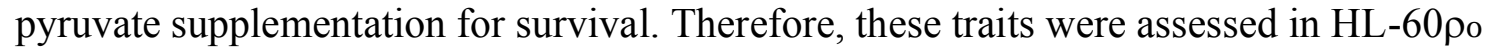
cells before conducting further experiments. Brightfield imaging shows HL-60 po cells maintain a suspension cell phenotype (Fig 3.1.A). Endpoint PCR products of both

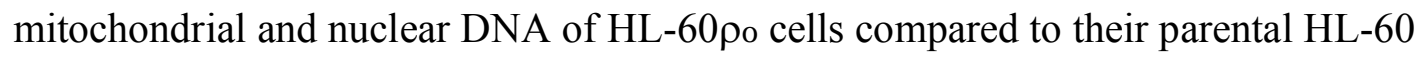
cells show that DNA from HL-60 po cells does not contain amplifiable mitochondrial DNA (Fig 3.1.B). An allele-specific-blocker qPCR will be used to detect donated

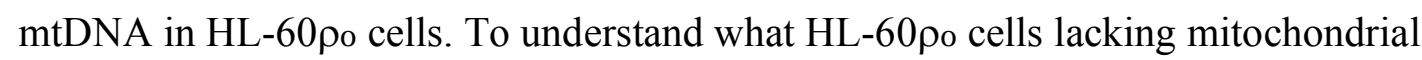

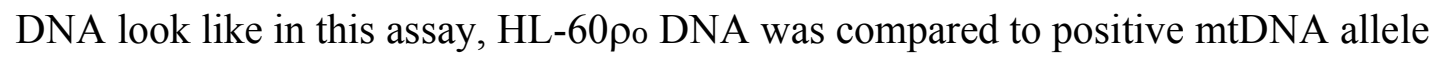
control HEL and two negative allele controls K562 and HL-60 - all possessing mitochondrial DNA. Results show that po cells amplify very late into the qPCR reactions and not as consistently compared to K562 and HL-60 WT which reproducibly amplify (Fig 3.1.C). This is likely due to the lack of any mtDNA binding template for

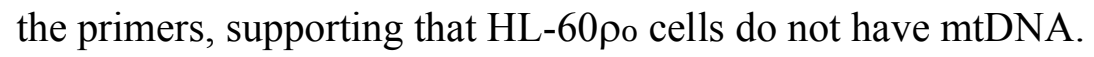

HL-60 po cells should also be dependent on pyruvate and uridine supplementation for survival in culture40. To test this, three media conditions were run side by side and viability of the cells was assessed every day for 7 days using Trypan Blue. When in complete media, HL-60 po cells maintained viability between 90-100\% (Fig 3.1.D). HL60 o cells, when deprived of pyruvate and uridine, slowly decreased in viability over the first 3 days, then a steep decline over days 3, 4 and 5 before a continued decrease in viability until less than $20 \%$ cells were viable after 7 days (Fig 3.1.D). As a positive control for death, the third condition contained HL-60 مo cells that were deprived of uridine, pyruvate and glutamine. Glutamine is an important amino acid that aids in producing energy via the mitochondria and biosynthetic products for cell proliferation 95. When HL-60 po cells are deprived of glutamine alongside pyruvate and glutamine their viability reduces similarly to uridine and pyruvate deprivation (Fig 3.1.D). 
A
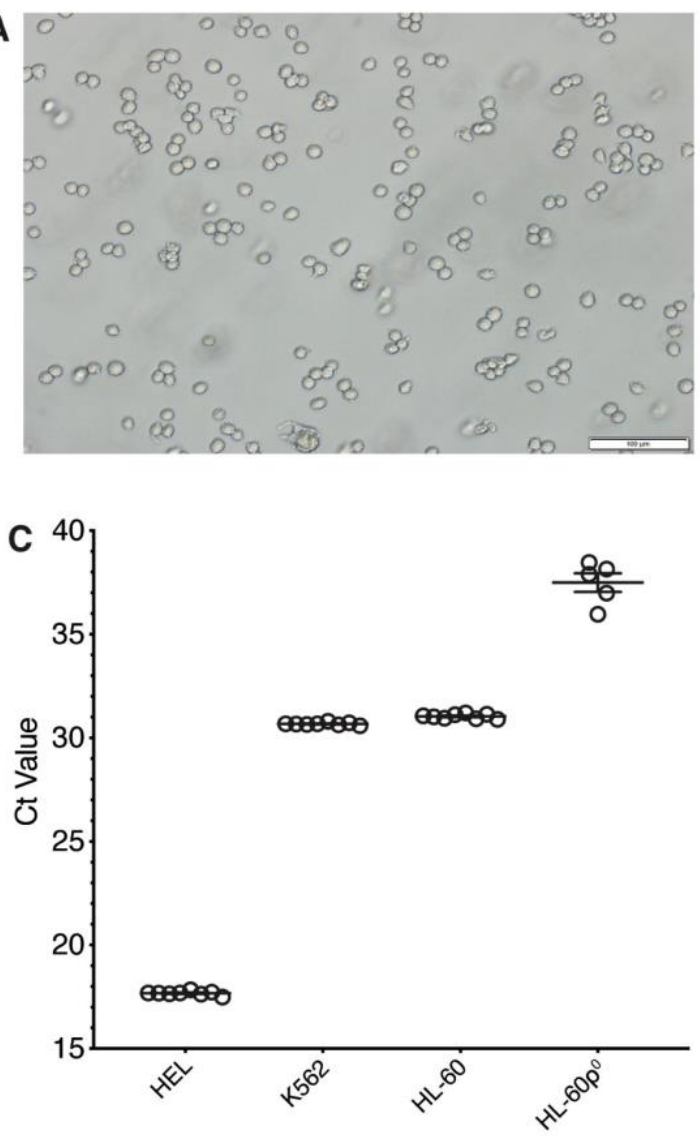

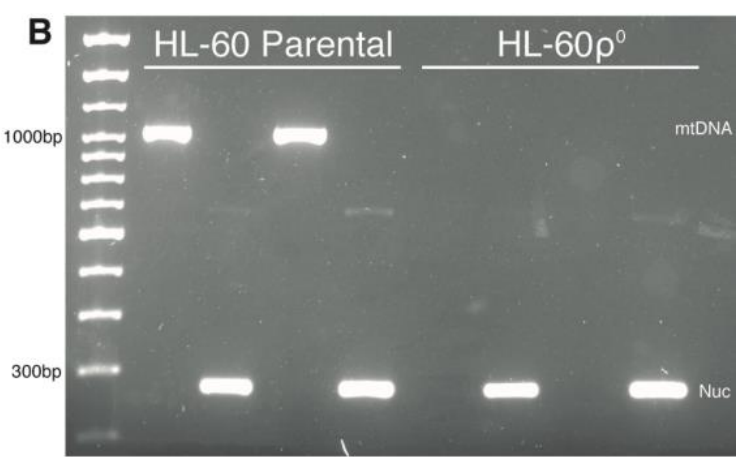

D

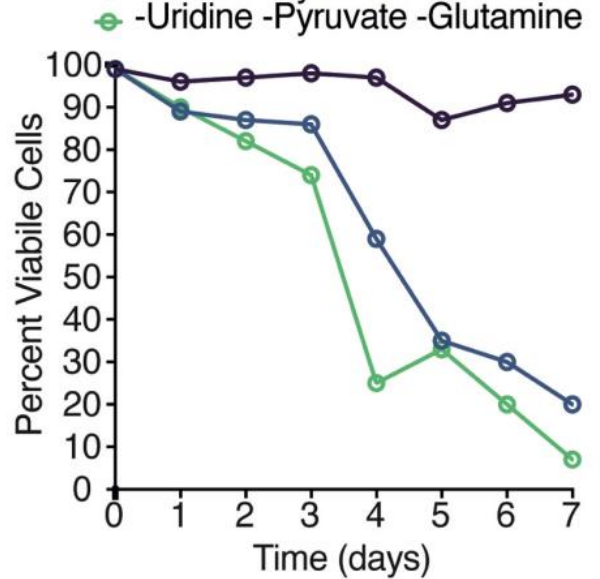

E

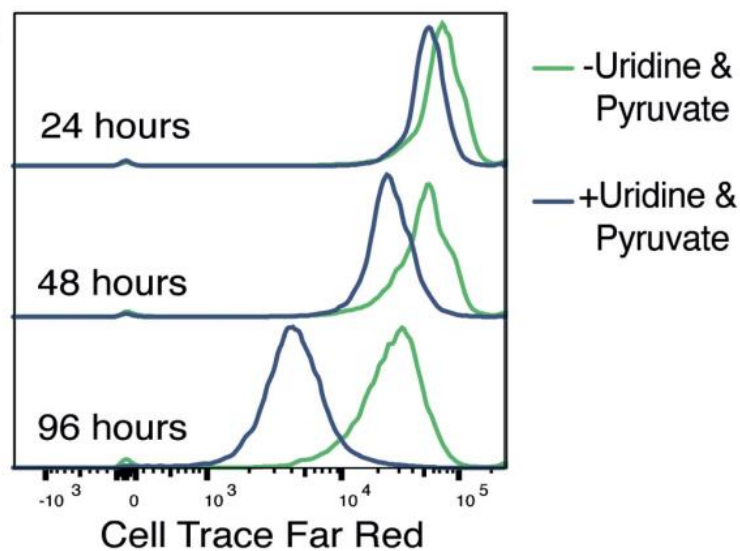

Figure 3.1: HL-60po cells lacked detectable mitochondrial DNA and had reduced viability and proliferative capacity when deprived of uridine and pyruvate.

(A) Brightfield image HL-60 po cells healthy in culture 40X magnification. (B) Endpoint mitochondrial (1024bp) and nuclear (268bp) PCR products for HL-60 parental and HL-60 oo cells. (C) qPCR amplification of ASBqPCR SNP195 showing characteristic amplification of HL-60 o cells, HEL, K562 and HL-60 cells (8 replicate wells each except where some did not amplify). (D) HL-60 po cells in complete media (+UP), deprived of uridine and pyruvate (-UP) or uridine, pyruvate and glutamine were Trypan Blue counted every day for 7 days to assess viability $(\mathrm{n}=1)$. (E) Proliferation histograms of HL-60 $\rho_{0}$ cells in complete media (blue line) or deprived of uridine and pyruvate (green line) stained with Cell Trace ${ }^{\mathrm{TM}}$ Far Red over 24, 48 and 96 hours $(\mathrm{n}=1)$. 


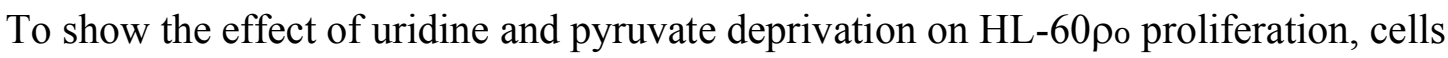
were labelled using Cell Trace Far Red. Cells in both complete and deprived culture conditions were analysed using flow cytometry as 24, 48 and 96 hours after initial staining. Histogram analysis of fluorescence intensity showed that HL-60po cells in complete media divide, and consequently reduce fluorescence, at least every 48 hours (Fig 3.1.E). By comparison, HL-60 مo cells that were deprived of pyruvate and uridine almost completely lost their proliferative capacity. By 96 hours they were almost a full $\log$ of fluorescence separate from the complete media counterpart (Fig 3.1.E). While

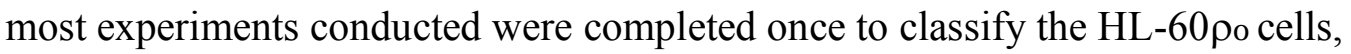
multiple different techniques were used which all support the same conclusion. The HL60 o cells used in this thesis did not have mtDNA and depend on pyruvate and uridine supplementation to survive as po cells should.

\subsubsection{HL-60 po cells are protected from declining viability when co- cultured with HEL cells or in HEL conditioned media.}

It is well known that cells communicate with each other. This communication can include mRNA, soluble factors such as cytokines, and even molecules such as pyruvate and uridine96-98. In order to try and separate general metabolic support from physical directed support - possibly by transfer of mitochondria - a conditioned media (CM) control was included in co-culture experiments by $0.22 \mu \mathrm{m}$ filtering 16-hour HEL

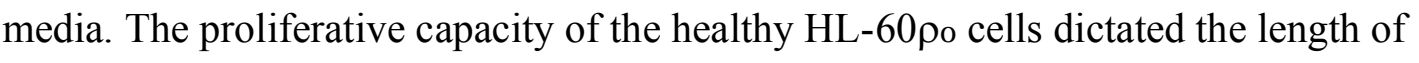
these experiments to allow separation from an unstained HEL population in co-culture. Therefore, co-cultures were run for up to 96 hours. In this section, a flow cytometry strategy was used to assess the viability of HL-60 po cells in six conditions: alone +UP, alone -UP, in conditioned media +UP, conditioned media -UP, co-culture with HEL +UP and co-culture with HEL -UP (Fig 3.2.A). HL-60 مo cells were seeded with HEL cells at a 2:1 concentration respectively and samples were taken from each culture condition at 24, 48 and 96 hours. Viability of Cell Trace labelled HL-60 po cells and unstained HEL cells was assessed using propidium iodide (PI), a dye that enters cells when their membrane becomes permeable due to cell death. As part of the experiment, I wanted to ensure the donor cell population, HEL, remained healthy and maintained high viability. Regardless of using a strict Propidium Iodide (PI) negative 

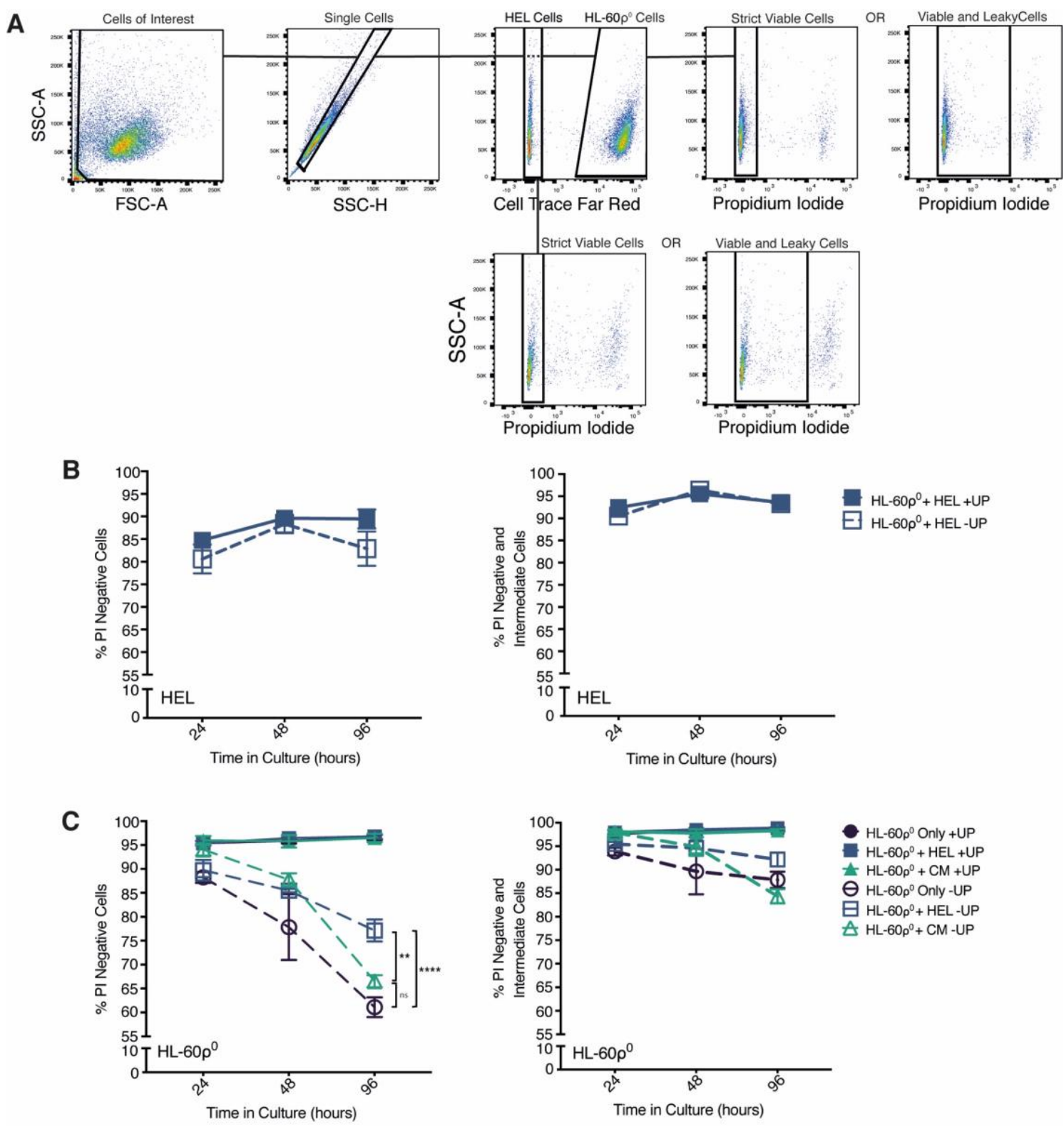

Figure 3.2: HL-60po cells are protected from declining viability when cocultured with HEL cells but not in HEL conditioned media.

(A) Gating strategy of Cell Trace Far Red stained HL-60 po cells showing both live cells only and viable and dying cells. HL-60 $\rho_{0}$ cells stained with Cell Trace Far Red were cultured alone, with unstained HEL cells or in HEL conditioned media with complete (+uridine and pyruvate (+UP)) and deprived (-UP) media for each condition. Flow cytometric data was collected at 24, 48 and 96 hours by taking samples. (B) Graphs showing viability of HEL cells in co-culture with HL-60 $\rho_{0}$ cells in complete (+UP) or deprived media (-UP). Strict Live cells on left graph and viable and dying cells included on right graph $(\mathrm{n}=3$ biological replicates) (C) Graphs showing viability of HL-60 po cells in each culture condition. Live cells only on left graph and viable and dying cells on right graph ( $\mathrm{n}=3$ biological replicates). Statistical analysis was performed using One-Way ANOVA with Tukey's post hoc-test showing mean \pm SEM. ${ }^{*} \mathrm{p} \leq 0.05, * * \mathrm{p} \leq 0.01, * * * * \mathrm{p} \leq 0.0001$. 
gate or including some PI intermediate (or dying) cells, the viability of HEL cells was maintained above $75 \%$ even in media without pyruvate and uridine (Fig 3.2.B).

As expected from prior results (Fig 3.1.E), HL-60 po cells in complete media would not reduce in viability regardless of the presence or absence of co-culture or $\mathrm{CM}$, they were included in viability graphs as controls (Fig 3.2.C). At 24 hours, using strict PI gating, there was a slight reduction in viability of each condition where uridine and pyruvate were removed. At 48 hours, this reduction increased most notably in the HL-60 po cells alone -UP. Additionally, only HL-60 $\rho$ o cells in conditioned media without UP showed

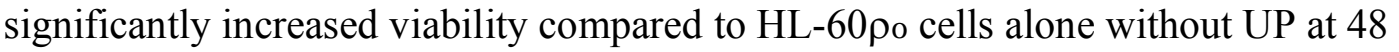
hours ( $\mathrm{p} \leq 0.05$ ). At 96 hours, HL-60 po cells in conditioned media without UP were no longer significantly higher in viability compared to HL-60 مo cells alone without UP. However, HL-60 po cells co-cultured with HEL without UP displayed between 10-20\%

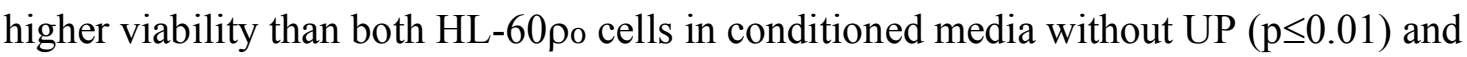
HL-60 po cells alone without UP $(\mathrm{p} \leq 0.0001)$. Upon including the intermediate PI stained cells, significant differences between all culture conditions were lost (Fig 3.2.C). It appeared that although conditioned media may have provided a slight amount of support it did not help survival of deprived HL-60 مo cells, compared to the direct coculture with HEL.

\subsubsection{HL-60 po cells do not recover proliferation capacity when co- cultured with HEL cells or in HEL conditioned media.}

Depriving HL-60 po cells of pyruvate and uridine caused them to stop proliferating as shown by lack of proliferation dye dilution over time (Fig 3.1.E \& F). Therefore, I wanted to assess if co-culture or conditioned media could rescue this phenotype. A gating strategy tailored to exclude cells negative for the Cell Trace dye - either HEL and/or any unstained HL-60 مo cells was used (Fig 3.3.A). Histograms of fluorescent

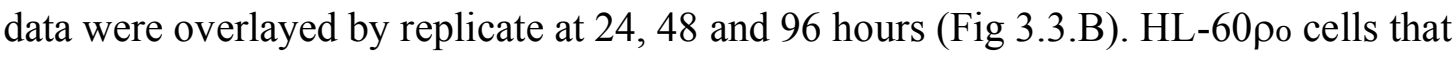
are not deprived of uridine and pyruvate successfully reduce peak fluorescent intensity

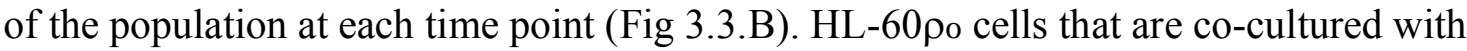
HEL or cultured in conditioned media and deprived have a similar peak fluorescence to

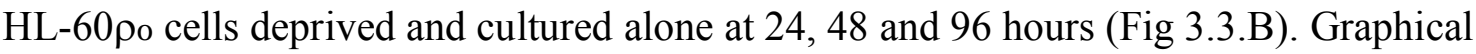




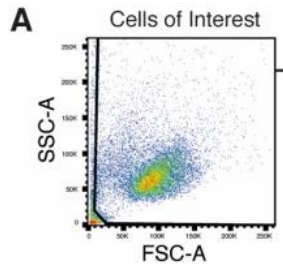

B

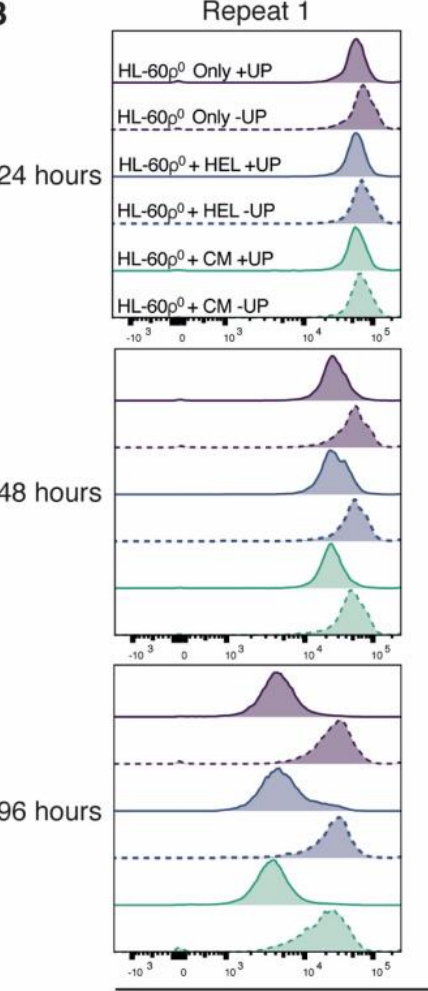

C

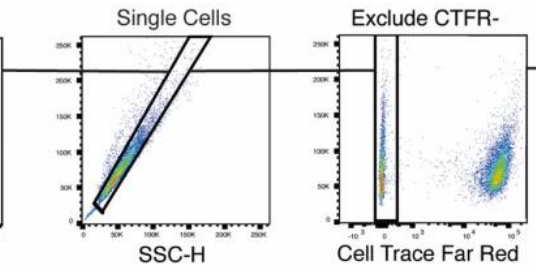

Repeat 2

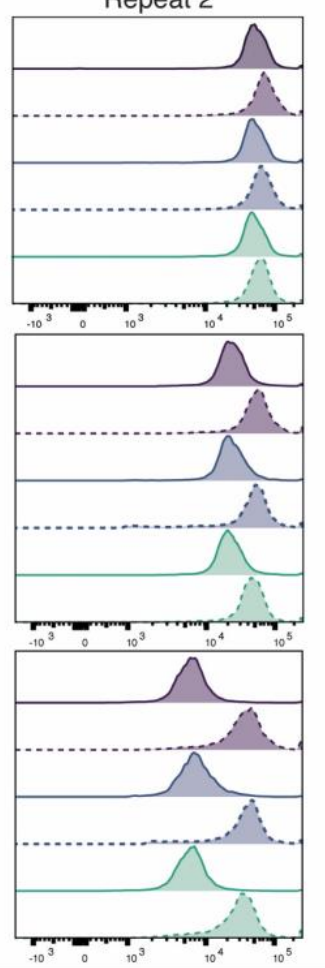

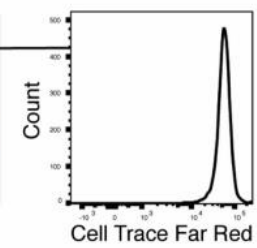

Repeat 3

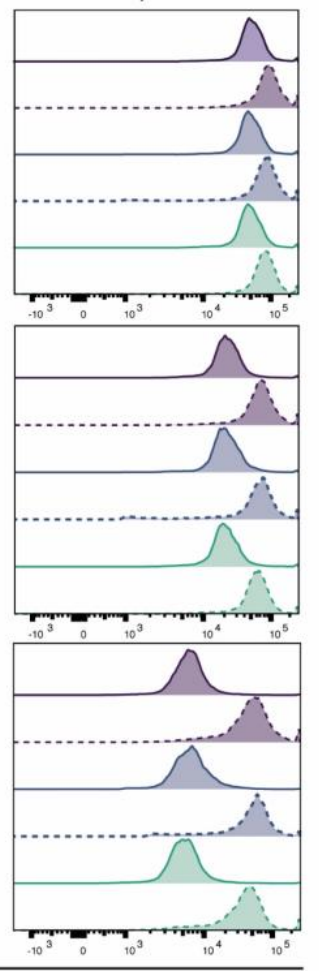

Cell Trace Far Red

- HL-600 Only +UP

․ㅡㄹ $\mathrm{HL}-600^{\circ}+\mathrm{HEL}+\mathrm{UP}$

$\neq \mathrm{HL}-60 \rho^{\circ}+\mathrm{CM}+\mathrm{UP}$

$\Theta$ HL-60 $\mathrm{\rho}^{\circ}$ Only -UP

日 HL-60 $\rho^{\circ}+\mathrm{HEL}-\mathrm{UP}$

$A \mathrm{HL}-60 \mathrm{O}^{\circ}+\mathrm{CM}-\mathrm{UP}$

\section{Figure 3.3: HL-60 po cells do not recover proliferation capacity when co-cultured with HEL cells or in HEL conditioned media.}

(A) Gating strategy to assess proliferation of Cell Trace Far Red stained HL-60 00 cells. (B) Three repeat experiments showing proliferation histograms of HL-60 00 cells at 24, 48 and 96 hours across 6 conditions. HL- $60 \rho 0$ cells cultured alone, in conditioned media or physical coculture with HEL cells with complete (+UP) and deprived (-UP) for each condition. (C) Graph representing the geometric mean fluorescence intensity (MFI) at each time point for each condition ( $\mathrm{n}=3$ biological replicates) calculated using FlowJo v10. Statistical analysis using Two-Way AVOVA and Tukey's Post Hoc Test showing mean \pm SEM reported no significance between the deprived conditions. 
representation of the geometric mean fluorescence intensity confirmed that no statistically significant difference occurs between HL-60 po cells deprived in co-culture or in conditioned media at any time point (Fig 3.3.C). Therefore, since the pattern of fluorescence was the same for all deprived conditions and different to healthy controls,

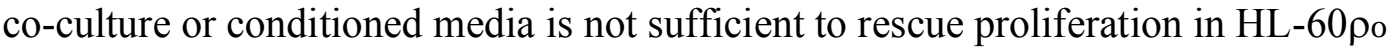
cells.

\subsubsection{HL-60 po cells do not uptake detectable levels of HEL mitochondrial DNA from HEL conditioned media.}

The Allele-Specific-Blocker qPCR (ASBqPCR) works by selectively amplifying a target DNA molecule, while excluding amplification of the background molecule 69. In

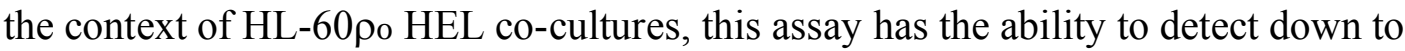
$0.001 \%$ target HEL mtDNA, which equates to 1 mitochondrial genome in $20 \mathrm{ng}$ of DNA. To detect mtDNA transfer in the HL-60 po cells, remaining cells from experiments used to assess viability and proliferation were pelleted for DNA extraction.

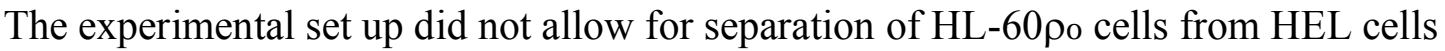

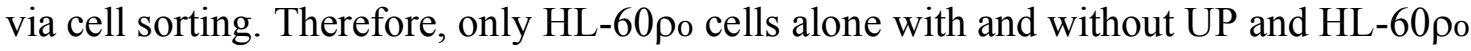
cells in conditioned media with and without UP were tested from these experiments using the ASBqPCR.

Standard curves of HEL DNA diluted in water were run with each replicate experiment to represent a percentage of target DNA (Fig 3.4.A\&B). A log-linear regression line was fit to the data in order to calculate the percent target DNA present given a specific $\mathrm{Ct}$ amplification cycle for each replicate (Fig 3.4.A\&B). The mean calculated target DNA for all four conditions or each replicate were below $0.001 \%$ HEL mtDNA and many of the 12 replicate wells did not amplify indicating no HEL template present (Fig 3.4.A\&B). Statistical analysis of the calculated \% target DNA for both replicates showed no significance between all conditions tested (Fig 3.4.A\&B). Interestingly, one experiment's conditions amplified earlier than the HL-60 po background. This was likely due to the inconsistent amplification that occurs when no mtDNA is present as shown previously in this chapter (Fig 3.1.C). When the percent target DNA is calculated for these samples, results still shows less than detectable levels of HEL mtDNA. Overall, these results indicate no detectable residual mtDNA present in the conditioned media 
A

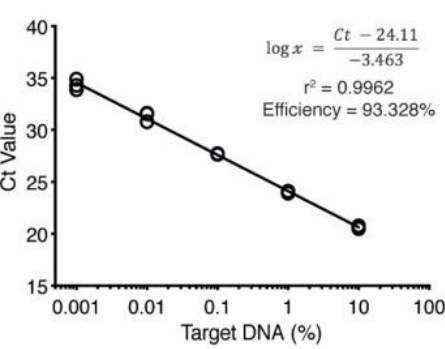

B

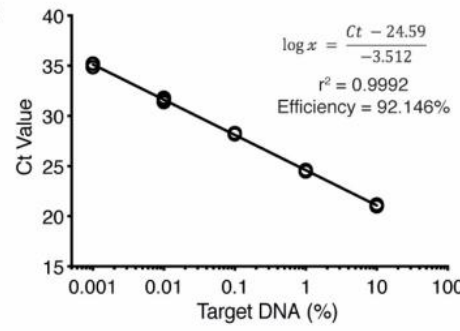

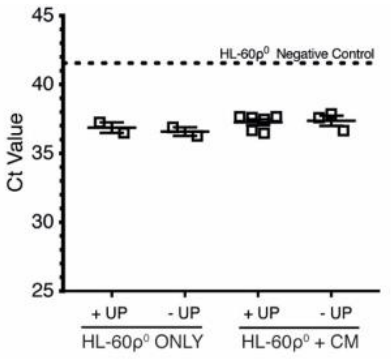

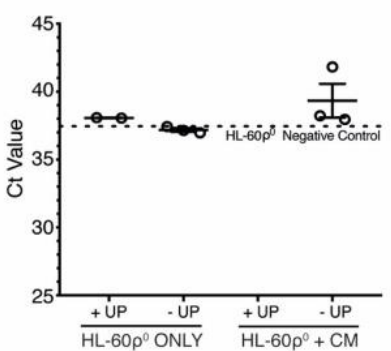

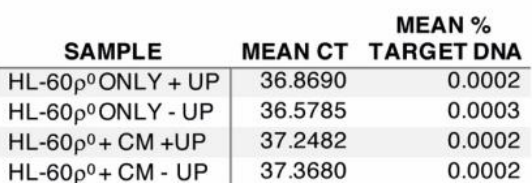

SAMPLE MEAN \% \begin{tabular}{l|rr}
\hline HL- $60 \rho^{0} \mathrm{ONLY}+$ UP & 38.0620 & 0.0001 \\
\hline
\end{tabular} $\begin{array}{lll}H L-60 \rho^{\circ} \mathrm{ONLY}-\mathrm{UP} & 37.1793 & 0.0003\end{array}$ $\mathrm{HL}-60 \rho^{0}+\mathrm{CM}+\mathrm{UP}$ Did not Amplify $\mathrm{HL}-60 \rho^{0}+\mathrm{CM}-\mathrm{UP} \quad 39.3323$

\section{Figure 3.4: HL-60 po cells do not uptake detectable levels of HEL mitochondrial DNA from HEL conditioned media.}

(A\&B) Left graph is a standard curve produced using qPCR amplification data. Data points were fitted with a log-linear line using least squares regression on Prism 8. The $\mathrm{r}_{2}$ value for the line is shown and the efficiency of the reaction as calculated by the Quantstudio software. Ct value - cycle at which fluorescence reached a threshold - is on the y axis and percent target DNA is on the $\mathrm{x}$ axis. Right graph shows individual replicate well Ct values for each experimental sample from duplicate experiments. Cell pellets were taken to extract DNA from after 96 hours in culture. For each sample up to 12 wells of DNA were tested and some did not amplify. HL-60 po cells co-cultured with HEL cells were not measured in these replicates because samples were not sorted into individual populations. Tables to the right of this graph show the mean $\mathrm{Ct}$ value for the samples and the calculated percent target DNA using the corresponding log-linear equation for that replicate. Replicate 1 is panel (A) and replicate 2 is panel (B). Statistical analysis was completed using One-Way ANOVA and Tukey's post-hoc test, graphs also showing mean \pm SEM. 


\subsubsection{Mitochondrial DNA is not transferred at a detectable level into HL-60 oo cells when physically co-cultured with HEL cells.}

Survival of HL-60 $\rho$ o cells when deprived of pyruvate and uridine was improved when in physical contact with HEL cells through co-culture. To determine if the co-culture

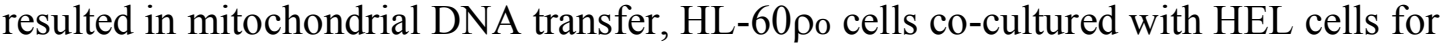
48 hours then sorted into separate populations using Cell Trace dye labelling. Firstly, the viability of both HL-60 po and HEL cells from the co-cultured samples was assessed using the shown gating strategy, comparing strict DAPI negative analysis and inclusion

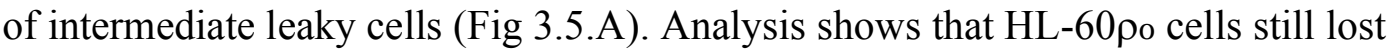
viability when deprived compared to their complete media counterpart to a similar degree seen at 48-hour point in previous time-course data (Fig 3.2.C \& Fig 3.5.B). The approximately $10 \%$ difference in deprived and non-deprived viability also did not change drastically between the two different gating strategies for DAPI negative cells (Fig 3.5.B). Interestingly, HEL cells varied more in their viability between media conditions, however, still maintained higher viability than the HL-60 po cells they were cultured with (Fig 3.5.B).

DNA analysis using the allele-specific-blocker qPCR corroborates with data shown earlier that there is no significant difference in levels of target mtDNA between HL60 o cells cultured alone or in conditioned media with or without uridine and pyruvate. The mean percentage of detected target mtDNA was between $0.0002 \%$ and $0.0006 \%$ for

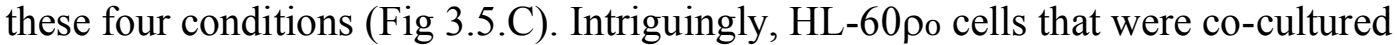

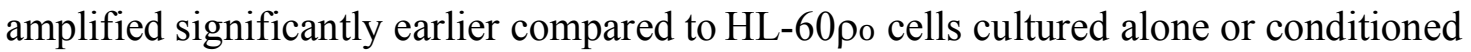
media samples. The deprived co-cultured sample amplified significantly earlier than the complete media counterpart $(\mathrm{p} \leq 0.0001)$ (Fig 3.5.C). This increase correlated to a $2.6 \mathrm{x}$ increase in detected HEL mtDNA from $0.3471 \%$ to $0.8950 \%$ in the deprived HL-60 po cells from this sample which may be indicative of mitochondrial DNA transfer into HL60 po cells.

To confirm if this increase detected was due to transfer of HEL mtDNA into HL-60مo cells, purity analysis of the sorted populations by flow cytometry was considered. Purity checks were done on a 1000 cell sample (Fig 3.5.D). The purity of the HL-60 po cells co-cultured with HEL in complete media was $98.6 \%$ and when co-cultured in deprived 
conditions the purity was $95.3 \%$. This correlates to a $3.3 \mathrm{x}$ increase in contamination. These were either labelled HEL cells or unlabelled fragments of HL-60 po or HEL cells. Contamination with even a small number of HEL cells will impact the sample $\mathrm{Ct}$ amplification in the ASBqPCR assay. Any evidence of mtDNA transfer into HL-60 مo cells would therefore need to be above $3.3 \mathrm{x}$ greater to show that there is transfer above the mtDNA present in contaminating cells. Therefore, with only a 2.6x increase in detected mtDNA in the deprived co-cultured sample it is not likely to be due to mitochondrial transfer but rather contamination.

This co-culture with sorted population DNA analysis was only completed once, however, the same issues of contamination are likely to plague all samples. Therefore, further replicates should not be completed until the correct techniques to use can be determined. 

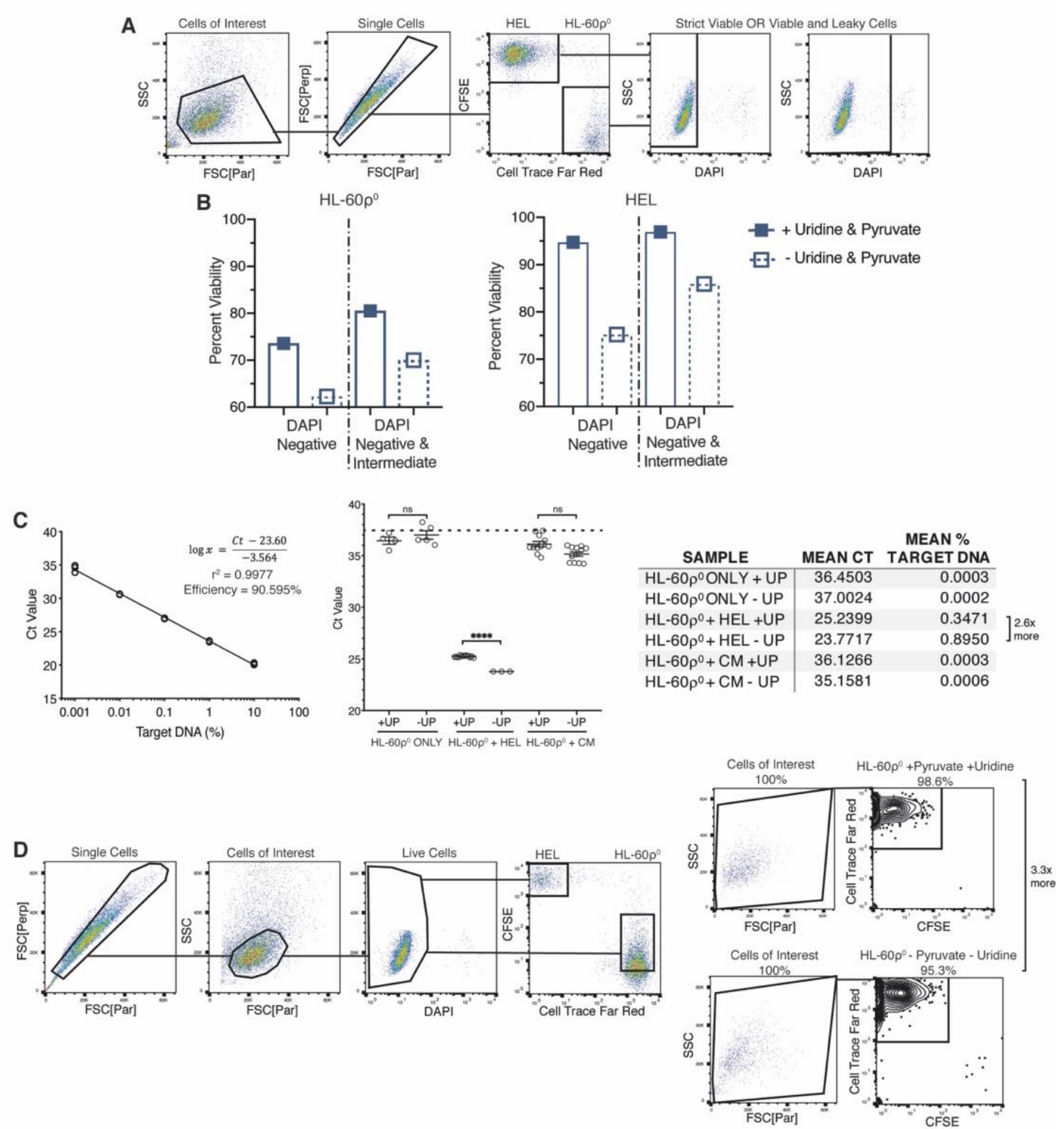

\section{Figure 3.5: Mitochondrial DNA was not transferred at a detectable level into HL-60 $\rho$ o cells when physically co-cultured with HEL cells.}

(A) Gating strategy used to assess DAPI negative or DAPI negative and intermediate cells of HL-60 $\rho_{o}$ and HEL cells in co-culture. (B) Viability of HL-60 po cells (left graph) and HEL cells (right graph) +or - uridine and pyruvate using both viability gating strategies $(n=1)$. (C) Single replicate of co-culture conditions where the HL-60 $\rho_{0}$ and HEL physical co-cultures were sorted into separate populations at 48 hours and cells were taken from other conditions also at 48 hours, pelleted and DNA extracted. Individual $\mathrm{Ct}$ values from between 3-12 replicate wells per sample are shown. The left graph shows the standard curve and equation fitted with a log-linear line using least squares regression in Prism 8, $\mathrm{r}_{2}$ and efficiency of the reaction calculated by Quantstudio 7 software. The graph to the right shows amplification $\mathrm{Ct}$ of the samples. The table to the right shows the mean Ct and calculated percent target DNA as per equation on the left graph. (D) Gating strategy for sorting out HL-60 مo and HEL physical co-cultures (left) and purity analysis of the complete (+UP) and deprived (-UP) samples (right). Statistical analysis was completed using One-Way ANOVA and Tukey's post-hoc test, graphs also showing mean \pm SEM. ${ }^{* * * *} \mathrm{p} \leq 0.0001$. $(\mathrm{n}=1)$ 


\subsection{Discussion}

Although previous data have been published showing that adherent po cells can uptake mitochondria and mtDNA transferred to them, the data shown in this chapter does not

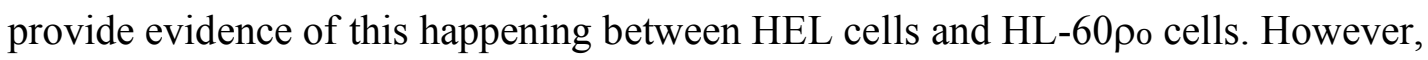
there is evidence suggesting the cells receive metabolic support to aid survival from metabolite deprivation but not enough to fully recover the proliferative capacity within the 96-hour time frame.

When working with po cells it is important to show that they lack any mitochondrial DNA and require supplementation for pyruvate and uridine to survive40. For cells to be considered po absence of mtDNA must be proven. I have successfully shown in this

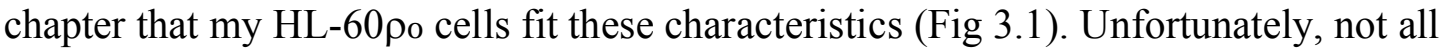
literature in this field can say the same. Some published research omits DNA evidence to support their po claim, not even included in the supplementary data99. Without this evidence, data provided that supports mtDNA transfer, without proving polymorphic differences between parental and donor cell mtDNA, cannot be considered valid.

Current detection of mitochondrial transfer heavily relies on visual techniques including microscopy via MitoTracker dyes to label mitochondrial networks, or ethidium bromide which can stain nuclear and mitochondrial nucleoli as well as indicate mitochondrial membrane potential68. These techniques are useful to produce beautiful images but lack real quantitation, and it can be difficult to discern if foreign labelled mitochondria in a cell is a real event68. Others have used genetic approaches such as sanger sequencing, or endpoint-PCR to detect mitochondrial DNA39,61-64,68. Basic sequencing methods, however, often lacks sensitivity. For example, Sanger sequencing often misses polymorphisms present below $15 \%$ and transfer is a rarer event in these conditions 100 . Alternative methods could include digital droplet PCR to detect mitochondrial transfer68. In order to bridge the gap in sensitivity of detection for molecular methods, I chose to use an assay called Allele-Specific-Blocker qPCR (ASBqPCR)69.

The use of HL-60 po cells with no mtDNA should allow for clearer detection of mtDNA transfer using this ASBqPCR assay because it has the ability to detect target mtDNA present above $0.001 \%$. However, trying to detect mtDNA transferred through physical

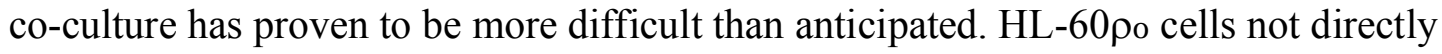


co-cultured with HEL did not contain detectable levels of mtDNA - less than $0.006 \%$

(Fig $3.4 \&$ Fig 3.5). This was not unexpected since the conditioned media was $0.22 \mu \mathrm{m}$ filtered and thus any free mitochondria $(1-10 \mu \mathrm{m})$ or microvesicles $(50-1000 \mathrm{~nm})$ would be removed101. It is also unlikely that any free mtDNA would transfer into cells due to a lack of data supporting direct mtDNA transfer without a mitochondrion or vesicle containing it.

I have shown that the assay can reliably detect small amounts of target DNA. Consequently, the purity of sorted samples for detection of mitochondrial DNA is of upmost importance. Unfortunately, the samples tested in this chapter were not higher than $99 \%$ pure and this led to limitations of mtDNA transfer detection. The qPCR detection ratio needed to be greater than the $3.3 \mathrm{x}$ difference in contaminating cell number between samples, to demonstrate there was additional copies of HEL mtDNA present that had not come from contaminating cells. The results of the qPCR assay did not surpass this limit for differences between deprived and non-deprived conditions and thus the differences are most likely due to contaminating HEL cells rather than mitochondrial transfer.

To confirm that the contaminating cells were HEL and not un-labelled HL-60 po cells, I would require a cell-type specific nuclear PCR control on the same DNA sample. Throughout the course of my Master's I explored a few different ways to distinguish between the cell lines genetically. These included Short Tandem Repeats (STR) where there were some differences in repeat length of nuclear DNA between cells, and explored the possibility of using a Y chromosome assay to distinguish between the cell lines. Unfortunately, the HEL cell line has not retained its Y chromosome, and the STRs were difficult to separate enough and were not sensitive enough because of this (data not shown). Future research in this project should focus on developing a nuclear control to draw definitive conclusion from the data. Using an in-situ method of visual DNA amplification called Rolling Circle Amplification should be considered as an alternative. It would remove the variable of sort purity and can include innate nuclear controls, but may be difficult as these suspension cells do not grow on slides easily and may not stay fixed during the process.

No data has been published to date showing cells donating mitochondria to their own cell type and the focus of this thesis is to determine if erythroblasts can transfer 
mitochondria between themselves. Most research into mitochondrial transfer focuses on two main donor sources, mesenchymal stem cells (MSC)54-56,58,102 and bone marrow derived stromal cells (BMSC)60-63. Erythroblast-like cells might not be able to transfer mitochondria, which could be another reason why transfer may not have occurred to a convincing level. Use of MSCs may be useful to consider in future experiments of this context.

Postulated mechanisms of transfer include microvesicle, exosomes, cell fusion but most research has focused on tunnelling nanotubules 103. Suspension cells in vitro may not find themselves capable of generating tunnelling nanotubules as readily as other cells and may have to rely on other methods such as microvesicles. Cell density and proportions of donor cells are also important. it may have been better suited to seed HEL cells at a lower concentration, it may also help avoid any results being skewed by trophic support. Mitochondrial transfer has been seen through xenografts of mice and suspension leukemia cells61,62. Inside bone marrow, cells are at a comparatively high density to in vitro, thus they are in closer proximity and could increase chances for transfer of mitochondria to occur.

Co-culturing HL-60 po cells with HEL cells resulted in an increase in viability compared to culturing cells alone when in deprived media, as did culturing in HEL conditioned media but not to a statistically significant level (Fig 3.2). It has been published previously that cells have the ability to produce growth factors, cytokines and molecules that provide support for survival and affecting the function and metabolism of cells around them96-98,104. There are two particular cell types that this is well characterised in - mesenchymal stem cells and glial cells. Interestingly, glial cells have the ability to produce and secrete pyruvate into the surrounding environment to increase survival and reduce injury to neuronal cells96,97. Pyruvate and/or uridine may be secreted by HEL cells into the media in small amounts, leading to the small increase in survival seen with conditioned media. When the HEL and HL-60 po cells were able to communicate in coculture, there was enough support to sustain survival to a greater extent.

Cell-cell signalling can have important effects on trophic support98. Mesenchymal stem cells have been shown to produce different growth factors at greater levels when cultured with serum from a patient with a stroke compared to a control patient's serum98. These stroke-serum activated MSC also improved angiogenesis and 
neurogenesis when injected into mice98. Differences in signals received by the donor cells is likely to explain the disparities in the results between the conditioned HEL media and the physical co-culture. Regardless of the communication between the stressed HL-60 po cells and donor HEL cells, the support was not enough to recover the HL-60 مo cells proliferative capacity (Fig 3.3). In future, it would provide valuable information to explore the metabolites that may be present in the conditioned and cocultured media. It may also be useful to leave the cultures for a longer period of time to see if the HL-60 po cells could eventually repopulate their mitochondria with donor mtDNA to a detectable level. Repopulation of mitochondrial genomes in po cells will rely on communication, termed cross talk, between the mitochondria and the nucleus 105 . Since $\rho$ o cells have survival without mtDNA for an extended period of time, the communication between mitochondria and nucleus will be altered and will take time to restore.

\subsection{Conclusion}

Mitochondrial transfer between erythroblast-like cells has not been shown previously. HL-60 po cells were used to first examine this. Their lack of mtDNA meant detection of transferred mtDNA would be feasible, and their dependence on pyruvate and uridine was a simple method to induce stress. In this chapter, I showed that it was unlikely that mitochondrial DNA had been transferred to a detectable level in this system. There was a negligible effect of conditioned media or physical co-culture on proliferation of HL60 o cells, however co-culture did significantly increase viability of HL-60 مo cells after 96 hours. 
4 Investigation of mitochondrial transfer between human bone marrow cell lines in vitro 


\subsection{Introduction}

Cells without mtDNA, po cells, are a unique model in which to identify transfer of mitochondria as simple analysis outputs can be used. For example, previous research has used Sanger sequencing to detect presence of polymorphisms present in donor mitochondrial genomes when tumours, grown from 4T1 $\rho$ o cells, were excised from mice39. Mitochondrial transfer and propagation within cells has also been shown through staining of recovered mtDNA via ethidium bromide previously lost in po cells39. po cells can, as previously demonstrated, be deprived of pyruvate and uridine to injure and promote transfer of mitochondria39,42,64,99. If cells do have existing mtDNA present, identifying transfer of mitochondria is not as straightforward.

Mitochondrial transfer has been investigated previously using cells replete for mtDNA 54-58,61-63. However, bone marrow studies have used a variety of injuries mostly centred around chemotherapies to induce or increase transfer of mitochondria. For example, two key studies focused on the use of chemotherapeutic injuries for mitochondrial transfer between human and mice using xenograft models 61,63 . These were acute myeloid leukemia (AML) cells treated with the nucleoside analogue Cytarabine61,72, and multiple myeloma cells treated with Bortezomib which inhibits proteasomes63,106. One study also showed the ability of hydrogen peroxide to increase mitochondrial transfer 62 . This was used to illustrate that the balance of oxidative stress within cells, a ubiquitous and important process, is involved in transfer of mitochondria63. Using chemotherapies supports the concept that mitochondrial transfer is important in cancer therapy resistance, while using hydrogen peroxide illustrates the key innate pathways that are involved in the process.

Erythroblasts are dependent on iron in production of heme to allow erythrocytes to function, a process which requires mitochondria34. Therefore, the iron chelator, Deferoxamine, is an interesting compound to investigate potential for mitochondrial injury and induction of mitochondrial transfer, which has not been tested in any previous research 49,50. A chemotherapy that is known to injure the mitochondria by intercalating with double stranded DNA is Cisplatin 57. Cisplatin has been shown to induce transfer of mitochondria from mesenchymal stem cells to neural stem cells to aid their recovery57. However, these treatments have not been explored in inter-erythroblast mitochondrial transfer. 
In order to explore inter-erythroid transfer of mitochondria, three erythroid-like cell line were used: K562, HEL and HL-60 cells. These cell lines all contain their own collection of mitochondrial genomes, as opposed to the po phenotype. Both deferoxamine and cisplatin were investigated as potential inducers of mitochondrial transfer between these cancer cell lines. The experimental determinant for mitochondrial transfer, as with the previous chapter, was the molecular Allele-Specific-Blocker qPCR assay.

\subsection{Aims}

1. Identify sequence polymorphisms that distinguish between HEL, K562 and HL60 cell lines.

2. Develop a molecular Allele-specific-blocker qPCR assay to leverage the polymorphisms identified.

3. Determine a concentration of deferoxamine, and of cisplatin, for use in coculture experiments.

4. Assess differences in recovery of cell viability in co-culture after treatment from deferoxamine or cisplatin.

5. Assess the presence of mitochondrial transfer using the ASBqPCR assay after deferoxamine or cisplatin treatment. 


\subsection{Results}

\subsubsection{HEL cells contained low levels of iron and HL-60 cells expressed CD38}

Deferoxamine (DFO) will remove iron present in the treated cultures via chelation 49,50. Therefore, HEL, K562 and HL-60 cell samples were cytospun onto slides and sent to Wellington Hospital SCL to have a routine Perl's stain performed to identify if the cells have any iron deposits naturally in culture. A positive control bone marrow slide was stained alongside the cell line samples and an image from the SCL lab was provided for comparison. From this control image the cells and extracellular matrix stain various red and brown shades whereas the iron clusters stain blue (Fig 4.1.A). Neither HL-60 (Fig 4.1.B) or K562 (Fig 4.1.C) displayed any blue staining that would indicate iron clustering. Interestingly, the stain illuminates the presence of dividing cells. Dividing cells had lighter red cytoplasm with a distinct dark red staining of chromatin condensing and examples of cells in anaphase and metaphase. Conversely, HEL cells displayed low amounts of positive blue iron staining (blue arrows, Fig 4.1.D). The staining resembled the control image where the blue smears do not appear to be contained fully by the cells. A select few of the HEL cells in a wider culture population appeared to have iron stores present and may indicate that this cell line will be affected more by deferoxamine treatment than the others.

Primary erythroblast cells have been shown to express CD38 particularly at early stages of erythropoiesis. CD38 is also implicated increasingly in literature in the formation of tunnelling nanotubules in mitochondrial transfer 63,107. HEL, K562 and HL-60 cell lines can be used as models of erythroblasts so their CD38 expression was checked using flow cytometry via a standard gating strategy (Fig 2.1). Results showed that classical erythroid lines HEL and K562 did not express any CD38 compared to the basal level of signal from the FMO control (Fig 4.1.E). Comparatively, the HL-60 and HL-60مo populations expressed CD38 on all cells in the culture (Fig 4.1.E). This experiment was only completed once, therefore expression may fluctuate due to length of time in culture. However, the results still form a good indication of whether the cells 
A

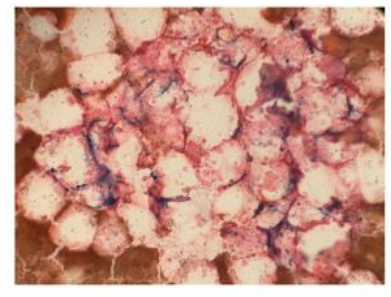

B
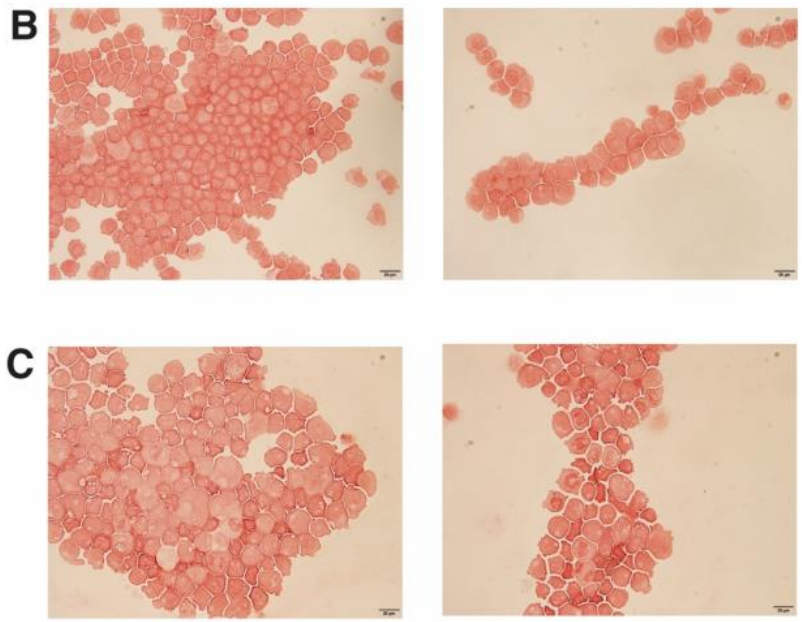

D
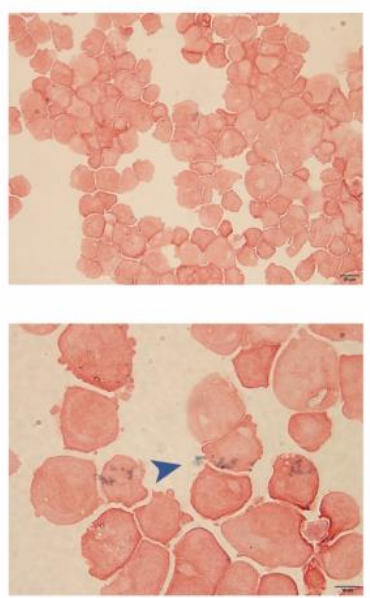
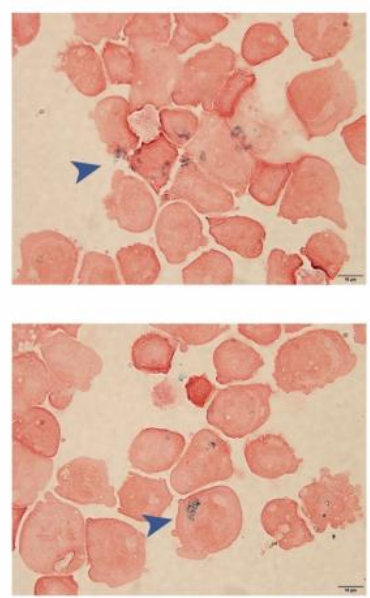

E

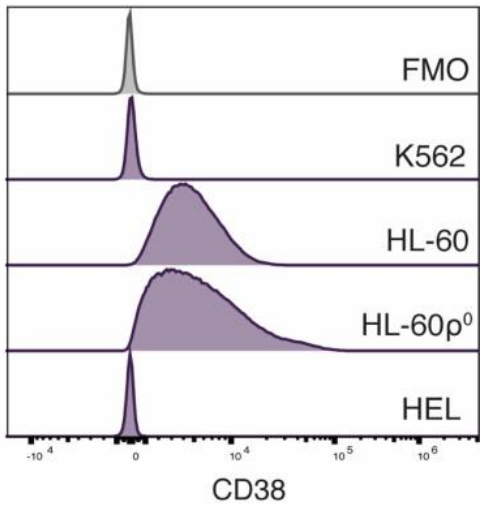

$\mathbf{F}$

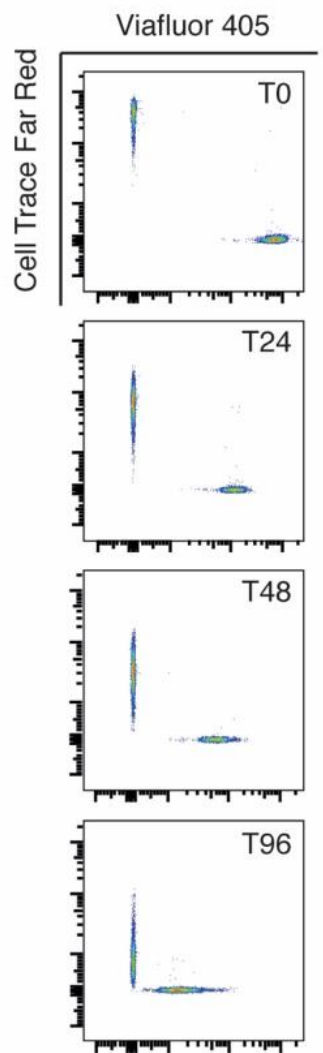

Figure 4.1: HEL cells contained low levels of iron and HL-60 cells expressed CD38.

(A) Positive control image of Perl's stain taken by Wellington Hospital SCL on an Olympus BX45 20X objective of bone marrow stained in parallel with the cell lines. Blue areas indicate presence of iron. $(\mathrm{n}=1)$ (B) Two representative images taken at 40x of Perl's stained HL-60 cells. No blue staining was seen. (C) Two representative images taken at 40x of Perl's stained K562 cells. No blue staining visible. (D) Four images of Perl's stained HEL cells. Top left image was taken at 40x objective and is representative of the overall level of positive staining. Top right and bottom two images were taken at 100x objective showing three separate examples of positive Perl's stain (blue arrows). (E) Histogram analysis of FMO control and 4 cell lines K562, HEL, HL-60 and HL-60 po derivative for CD38 staining detected via flow cytometry. (n=1) (F) Time course over 96 hours of HEL (Cell Trace Far Red) and HL-60 (Viafluor 405) in co-culture together to visualise the limit of separation for future co-culture experiments. (representative data from 2 experiments) A standard flow cytometry gating strategy was used for (E) and (F) as shown in Figure 2.1 in the Materials and Methods. 
express CD38. These results were considered when deciding cell line combinations for co-culture experiments. Cell Trace proliferation dyes are the most efficient way to label these cell lines to separate out after co-culture. Because the signal from these dyes will dilute with proliferation, a time course was conducted to determine the optimal length of time cells could be left in co-culture to allow for adequate separation of populations. Flow cytometry analysis showed division of cells roughly every 24 hours and thus fluorescence intensity decreased accordingly (Fig 4.1.F). Ultimately, a co-culture between 48 and 72 hours was likely to yield the best balance of time in co-culture and level of fluorescence for separation via flow cytometry (Fig 4.1.F).

\subsubsection{Two single nucleotide polymorphisms were leveraged to design an Allele-Specific-Blocker qPCR assay to detect HL-60 mtDNA transfer}

To detect mtDNA transfer between the cell lines, molecular assays targeting polymorphisms needed to be developed. Sanger sequencing of the amplified mitochondrial D-loop region of the genome, also known as the hypervariable region, identified two polymorphisms separated by a single nucleotide on the HL-60 genome compared to HEL and K562 (Fig 4.2.A). The two polymorphisms, m150T $>\mathrm{C}$ and m152C $>\mathrm{T}$ were used to design an allele-specific-blocker qPCR assay specific for HL-60 mtDNA.

An artificial spike-in of target HL-60 mtDNA into background HEL and K562 DNA was used to determine the limits of detection for the ASB-qPCR assay. When HL-60 mtDNA was in a background of HEL DNA the limit of detection was $0.001 \%$ target mtDNA, however when HL-60 mtDNA is in a K562 DNA background the limit of detection changes to $0.01 \%$ (Fig 4.2.B). While the limit of detection varied depending on the context of the co-culture, the ability to detect target mtDNA by this assay is still high. The HL-60 mtDNA allele-specific-blocker qPCR involves two allele variants at positions 150 and 152 on the mitochondrial genome in the designs of the allele-specific and blocker primers. The common reverse primer and hydrolysis probe bind to both the on and off target mtDNA genomes, however the off-target template is exponentially diluted from the reaction and detectable fluorescence only results from the target HL-60 mtDNA template (Fig 4.2.C). 
A \begin{tabular}{r|r|} 
K562 $^{5}$ & GATTCCTGCCTCATCCTATTATTTAT \\
HEL & GATTCCTGCCTCATCCTATTATTTAT \\
HL-60 & GATTCCTGCCTCATTCCATTATTTAT
\end{tabular}
B
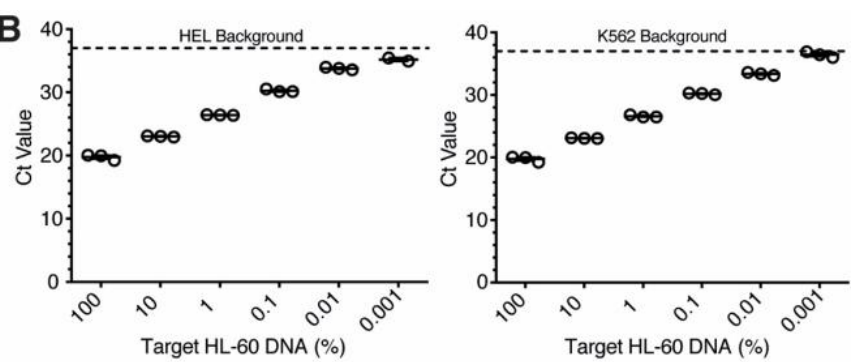

C

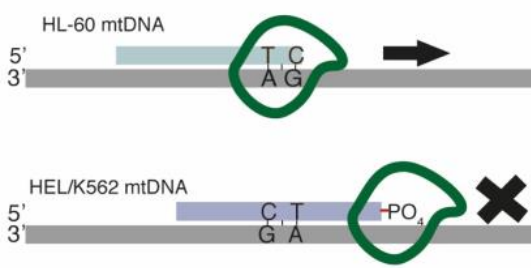

Allele Specific Primer (On Target)

Allele Specific Blocker (Off Target)

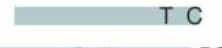

$\mathrm{C} \mathrm{T} \quad-\mathrm{PO}_{4}$
Common Reverse Primer Hybridisation Fluorescent Probe $\sqrt{20}$

Figure 4.2: Two single nucleotide polymorphisms were leveraged to design an Allele-Specific-Blocker qPCR assay to detect HL-60 mtDNA transfer.

(A) Sanger sequencing results from the displacement loop region of mtDNA of K562, HEL and HL-60 mtDNA. At this specific region two polymorphisms separated by one nucleotide were identified $\mathrm{m} .150 \mathrm{~T}>\mathrm{C}$ and $\mathrm{m} .152 \mathrm{C}>\mathrm{T}$ on the HL-60 mitochondrial genome that were not in K562 or HEL mitochondrial sequences. Representative of 2 forward and reverse sequencing runs. (B) Allele-specific-blocker qPCR standard curves detecting diluted amounts of target HL60 mtDNA in controlled off-target background HEL or K562 DNA. The dotted horizontal line shows the Ct amplification an unmixed off-target background HEL or K562 samples amplified as the limit of detection. Representative data of 5 experiments. (C) Schematic depicting the design of the ASBqPCR for detecting HL-60 mtDNA in a HEL or K562 DNA background. The background polymorphism is bound by a blocker primer with a 3' phosphate group preventing extension by DNA polymerase, preventing release of fluorescence from the probe downstream. Both on- and off-target templates are bound by the common reverse primer. The target sequence is bound by a specific primer which allows DNA polymerase extension and release of fluorescence read by the qPCR machine. Adapted from Morian et al., 2009. 


\subsubsection{K562 were resistant to Deferoxamine reduction in viability and mitochondrial membrane potential}

To determine whether K562 or HEL would be co-cultured with HL-60, the effect of deferoxamine (DFO) on apoptosis was determined. Induction of apoptosis was determined using Annexin V and a viability dye (Zombie Near Infrared) alongside a further analysis of the mitochondrial membrane potential of each cell population using Mitoview Blue (Fig 4.3.A). The four populations investigated were cells in annexin negative cell death, late apoptotic, early apoptotic and healthy (non-apoptotic) cells. K562 were treated for 96 hours with deferoxamine in their culture media over 5 different concentrations from $10 \mu \mathrm{M}$ to $300 \mu \mathrm{M}$. Deferoxamine was dissolved in sterile water and minimal volumes of water were added to the media (less than $40 \mu \mathrm{L}$ in $10 \mathrm{~mL}$ media). Therefore, treated cells were compared to an untreated population rather than a vehicle control.

Healthy cells remained above $90 \%$ of the population when K562 cells were left untreated over 96 hours. Untreated K562 cells also maintained a population with equal size (FSC) and granularity (SSC) (Fig 4.3.B).

Neither $10 \mu \mathrm{M}$ or $25 \mu \mathrm{M}$ DFO increased apoptosis or annexin negative cell death compared to the untreated control cells over 96 hours of treatment. This was mirrored by the lack of change in size and granularity of K562 cells by either concentration.

Deferoxamine treatment at $50 \mu \mathrm{M}$ induced $30 \%$ apoptosis after 48 hours $(20 \%$ early and $10 \%$ late apoptosis). Apoptosis was further increased after 96 hours of treatment to $50 \%$, with $40 \%$ of cells in the late stage. As cells undergo apoptosis, they tend to reduce their cell size (FSC) and increase variability of granularity (SSC) 3. Development of this population can be seen by 48 and 96 hours of $50 \mu \mathrm{M}$ DFO treatment.

Similar numbers of cells entered apoptosis when K562 cells were treated with $100 \mu \mathrm{M}$ and $300 \mu \mathrm{M}$ DFO. At 24 hours, $15 \%$ of cell entered apoptosis, increasing to $40 \%$ at 48 hours and $70 \%$ at 96 hours. However, $300 \mu \mathrm{M}$ DFO consistently induced more cells in late apoptosis at each time point. 
Both 100 and $300 \mu \mathrm{M}$ increased the proportions of FSC and SSC changes seen in $50 \mu \mathrm{M}$ DFO treatment with an end result at 96 hours, where most cells shifted to the left. Notably, K562 cells show a distinct lack of annexin negative cell death induced at every DFO concentration (Fig 4.3.B).

To determine if deferoxamine had an effect on the mitochondria of K562 cells, Mitoview Blue staining was employed. Using this stain, mitochondria stain blue when their mitochondrial membrane is intact, and they lose fluorescence when mitochondrial membrane potential is lost.

Untreated K562 cells present with a clear positive population in their healthy population and early apoptotic cells showed bimodal population with high and low membrane potential (Fig 4.3.C). Late apoptotic cells, on the other hand, were mostly low for mitoview blue indicating they have lost their membrane potential, however some residual cells that maintained membrane potential could be visualised. Interestingly, in untreated K562 cells, the annexin negative cell death population also presented bimodal staining of Mitoview Blue.

When treated with $10 \mu \mathrm{M}$ and $25 \mu \mathrm{M}$ DFO, membrane potential did not change compared to the untreated K562 cells over 96 hours.

Subsequent increased concentrations of DFO $(50-300 \mu \mathrm{M})$ reduced the number of cells that maintained mitochondrial membrane potential, in a dose-dependent manner over 96 hours of treatment. Similarly, more cells in early apoptosis decreased their membrane potential with increasing DFO concentration. Residual late apoptotic cells and cells in annexin negative cell death that retained mitochondrial membrane potential were also reduced with increasing DFO concentration (Fig 4.3.C).

These experiments were completed a single time; however, they still provide indications of concentrations suitable for subsequent experiments. In sum, treatment with deferoxamine was able to induce apoptosis in K562 cells at concentrations higher than $25 \mu \mathrm{M}$ over 96 hours, however the level of injury to mitochondrial membrane potential was not at the same intensity. 


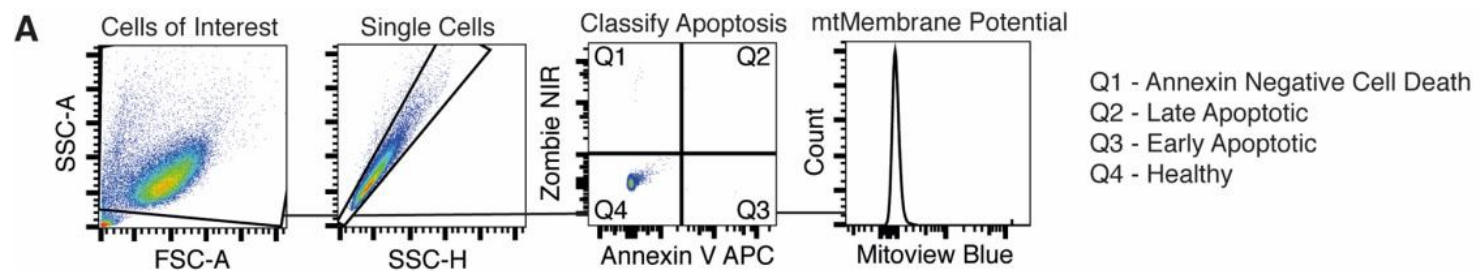

B
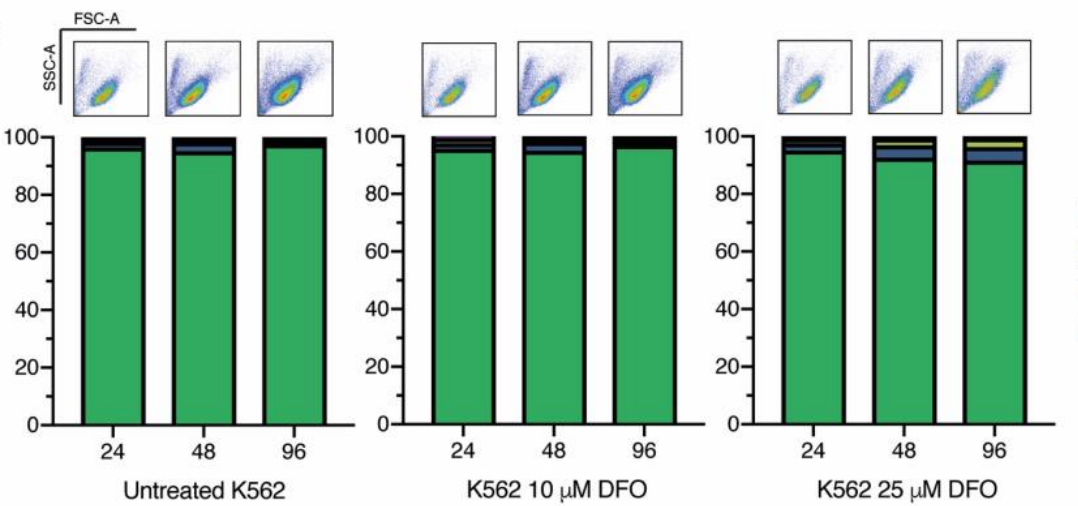

Annexin Negative Cell Death

$\square$ Late Apoptotic

$\square$ Early Apoptotic

$\square$ Healthy
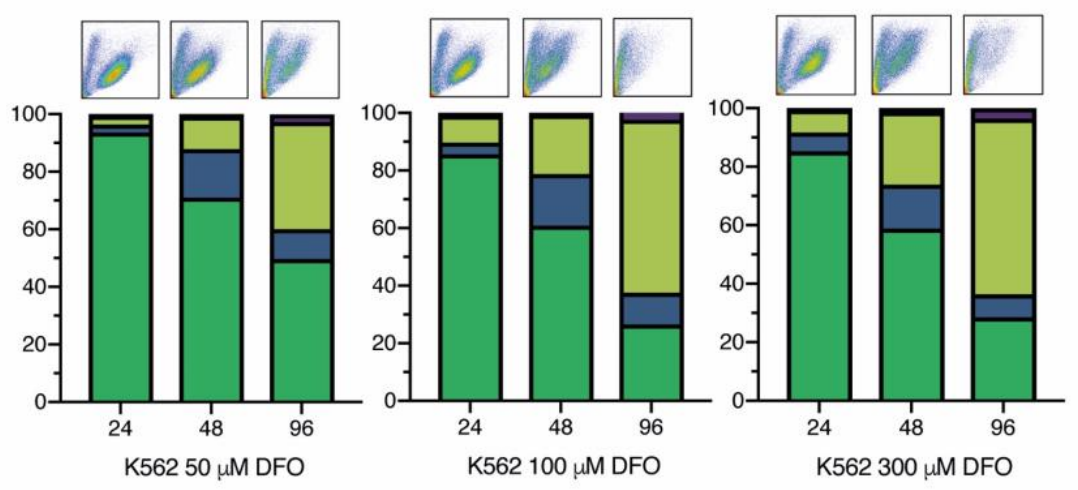

Figure 4.3: K562 were somewhat resistant to Deferoxamine reduction in viability and mitochondrial membrane potential.

(A) Flow cytometry gating strategy using to identify apoptotic stages and the mitochondrial membrane potential of the cells within those stages. (B) Deferoxamine titration graphs showing the four stages identified by an apoptosis panel: Annexin negative cells death, late apoptosis, early apoptosis and healthy (non-apoptotic). Above each column on each graph are FSC SSC plot showing morphology of cells at each time point measured. (C) Histogram overlays of 24, 48 and 96-hour Mitoview blue fluorescence analysis for each apoptotic population of cells in each DFO concentration. $(n=1)$ 
C

Annexin Negative

Late Cell Death

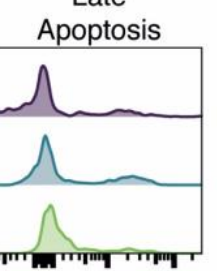

Early
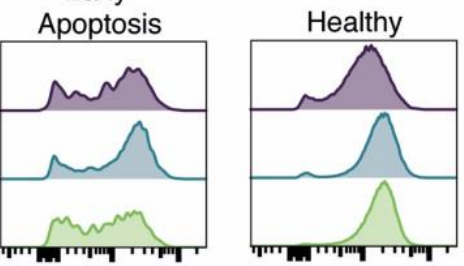

24 hours

48 hours

96 hours
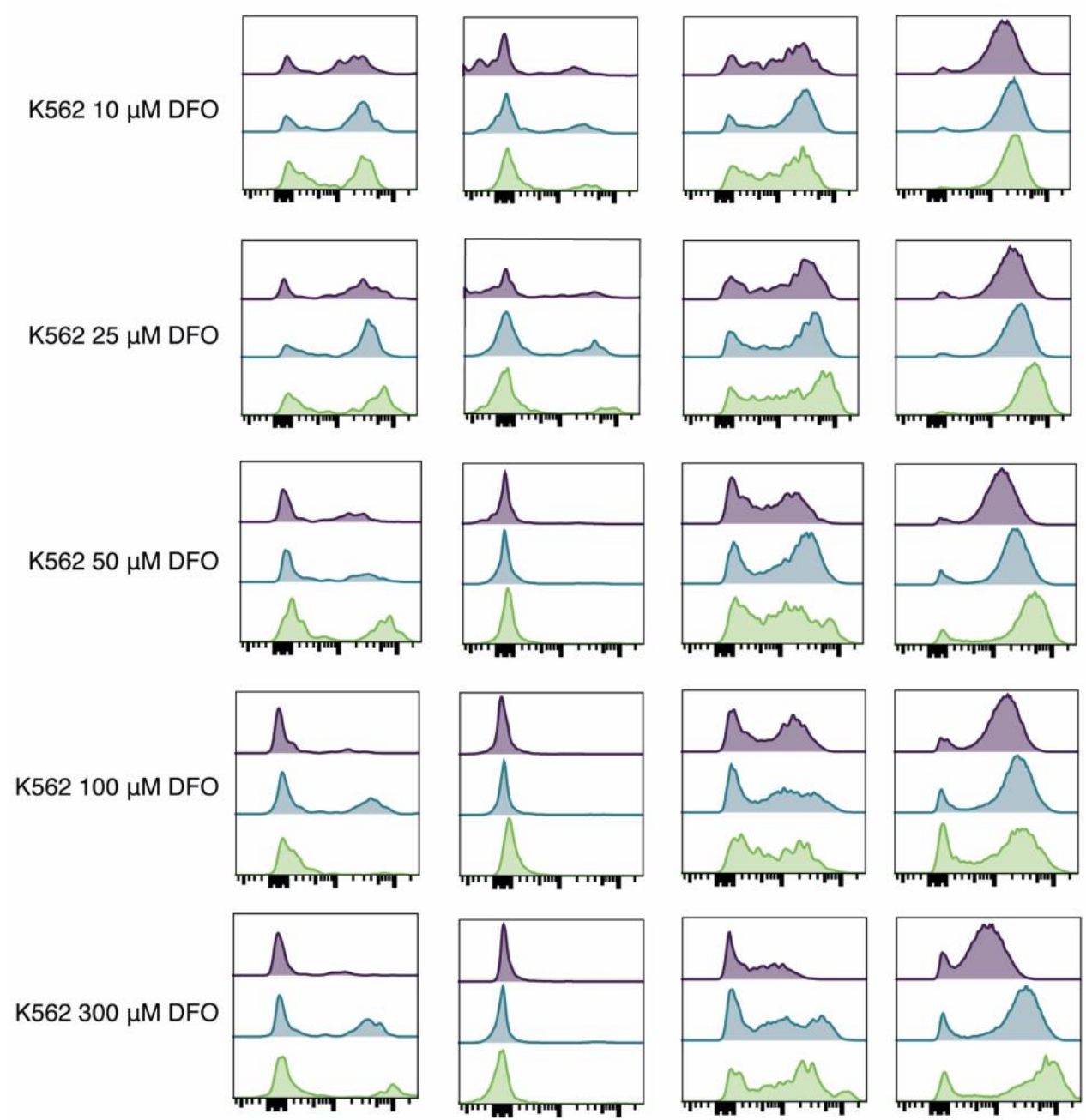

Mitoview Blue 


\subsubsection{HEL cells were sensitive to deferoxamine reduction in viability and mitochondrial membrane potential}

HEL cells were another potential recipient in mitochondrial transfer co-cultures that had a different mtDNA polymorphism to HL-60. The same titration of deferoxamine was conducted on these cells and the same analysis tools were used to assess apoptotic and mitochondrial membrane responses to the drug (Fig 4.4.A). An additional concentration of $200 \mu \mathrm{M}$ was included for these cells.

Untreated HEL maintained viability above $95 \%$ throughout the time course. Consistent with this, their FSC/SSC profile also remained the same as previous untreated cells.

Over 96 hours, treatment with $10 \mu \mathrm{M}$ DFO triggered small increases in apoptosis. Both early and late apoptosis increased collectively to $15 \%$ of the population $(5 \%$ and $10 \%$ respectively) by 9 hours. Interestingly, a basal level of 5\% cells in annexin negative cell death occurred at each time point. The granularity (SSC) of HEL cells began to increase correlating with the induction of apoptosis.

Twenty-five $\mu \mathrm{M}$ DFO treatment increased apoptotic populations of HEL cells. At 48 hours, $40 \%$ of cells entered cell death (20\% early apoptosis, $15 \%$ late apoptosis and $5 \%$ annexin negative cell death). At 96 hours, $80 \%$ had reached either late apoptosis or annexin negative cell death (40\% each). By 96 hours, HEL cells doubled their level of granularity (SSC) and halved their size (FSC).

Treatment with 50-300 $\mu \mathrm{M}$ DFO caused a concentration-dependent increase in early apoptosis at 24 hours $-5 \%$ at $50 \mu \mathrm{M}, 10 \%$ at $100 \mu \mathrm{M}, 15 \%$ at $200 \mu \mathrm{M}$ and $20 \%$ at 300 $\mu \mathrm{M}$. Late apoptotic cells increased by $15 \%$ at 48 hours for each concentration, reaching the highest proportion of 55\% at $200 \mu \mathrm{M}$ and $300 \mu \mathrm{M}$. Most notably, increasing DFO concentration induced annexin negative cell death in $70 \%$ of cells with $50 \mu \mathrm{M}, 80 \%$ of cells with $100 \mu \mathrm{M}, 90 \%$ of cells with $200 \mu \mathrm{M}$ and $95 \%$ of cells with $300 \mu \mathrm{M}$ by 96 hours. DFO treatment of 50-300 $\mu \mathrm{M}$ induced the same left shift of apoptotic cells of SSC FSC changes as $25 \mu \mathrm{M}$ DFO treatment (Fig 4.4.B). 
Mitochondrial injury caused by DFO was assessed using mitoview fluorescence of each apoptotic populations (Fig 4.4.A). Untreated HEL cells maintained consistent mitoview blue fluorescence and therefore, mitochondrial membrane potential compared to untreated K562 cells. By 96 hours, $10 \mu \mathrm{M}$ DFO treatment caused a small proportion of cells in each stage of cell death to lose their mitochondrial membrane potential. The mitochondrial membrane potential of cells that remained healthy were unaffected by 10 $\mu \mathrm{M}$ treatment.

Both healthy and early apoptotic cells exposed to DFO concentrations above $10 \mu \mathrm{M}$ lost mitochondrial membrane potential in a concentration-dependent manner by 96 hours. Interestingly, complete reduction in mitochondrial membrane potential was seen by 96 hours by $25 \mu \mathrm{M}$ DFO treatment in HEL cells that entered late apoptosis or annexin negative cell death, which was the maximal effect seen with all subsequent concentrations.

Overall, DFO had a more exaggerated effect on reducing the mitochondrial membrane potential in HEL cells, compared to K562 cells, even at lower concentrations. This response correlated with a sensitivity to concentrations about $25 \mu \mathrm{M}$ that lead to high levels of annexin negative cell death by 96 hours. It is possible that the indication of iron accumulation in HEL cells naturally in culture from Perl's stain (Fig 4.1.D) may be implicated in the sensitivity to DFO. For these reasons, HEL were a better candidate for DFO induced mitochondrial transfer and chosen for use in co-culture experiments. 
A
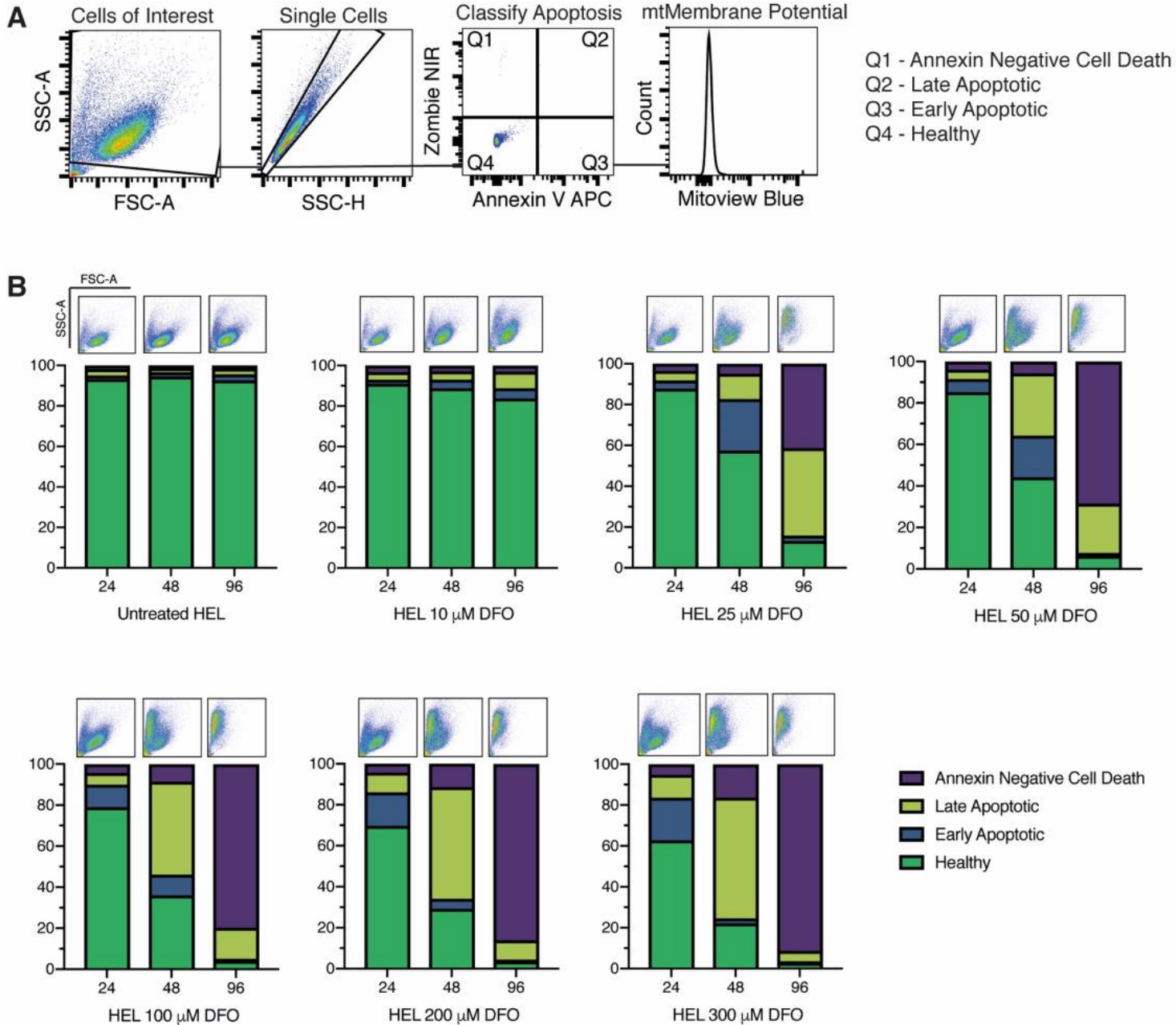

$$
\begin{aligned}
& \square \text { Annexin Negative Cell Death } \\
& \square \text { Late Apoptotic } \\
& \square \text { Early Apoptotic } \\
& \square \text { Healthy }
\end{aligned}
$$

Figure 4.4: HEL cells were sensitive to deferoxamine reduction in viability and mitochondrial membrane potential.

(A) Flow cytometry gating strategy using to identify apoptotic stages and the mitochondrial membrane potential of the cells within those stages. Same plots as shown in Fig 4.3. (B)

Deferoxamine titration graphs showing the four stages identified by an apoptosis panel: Annexin negative cells death, late apoptosis, early apoptosis and healthy (non-apoptotic). Above each column on each graph are FSC SSC plot showing morphology of cells at each time point measured. (C) Histogram overlays of 24, 48 and 96-hour Mitoview blue fluorescence analysis for each apoptotic population of cells in each DFO concentration. $(\mathrm{n}=1)$ 
C

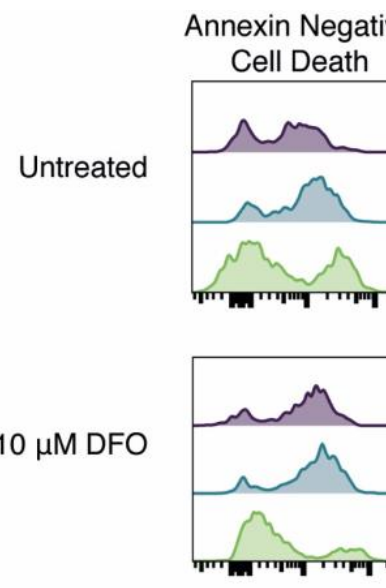

HEL $25 \mu M$ DFO

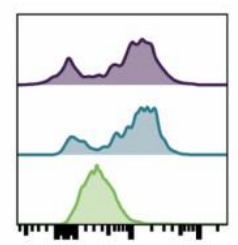

HEL $50 \mu M$ DFO

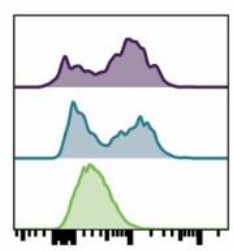

HEL $100 \mu$ M DFO
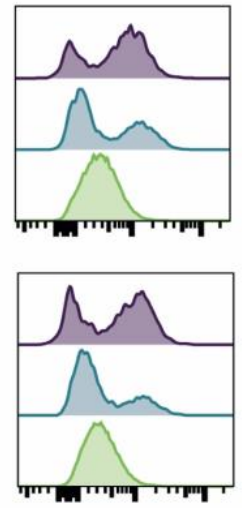

HEL $200 \mu M$ DFO

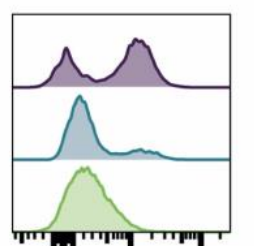

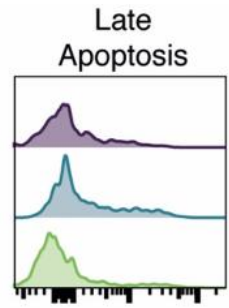
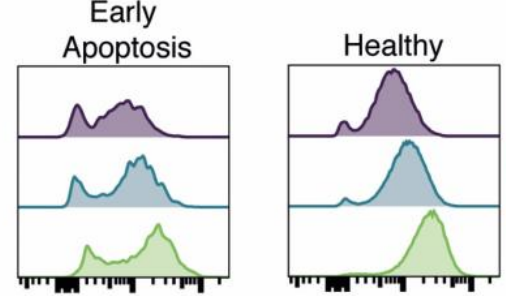

$\square 24$ hours

$\square 48$ hours

$\square 96$ hours
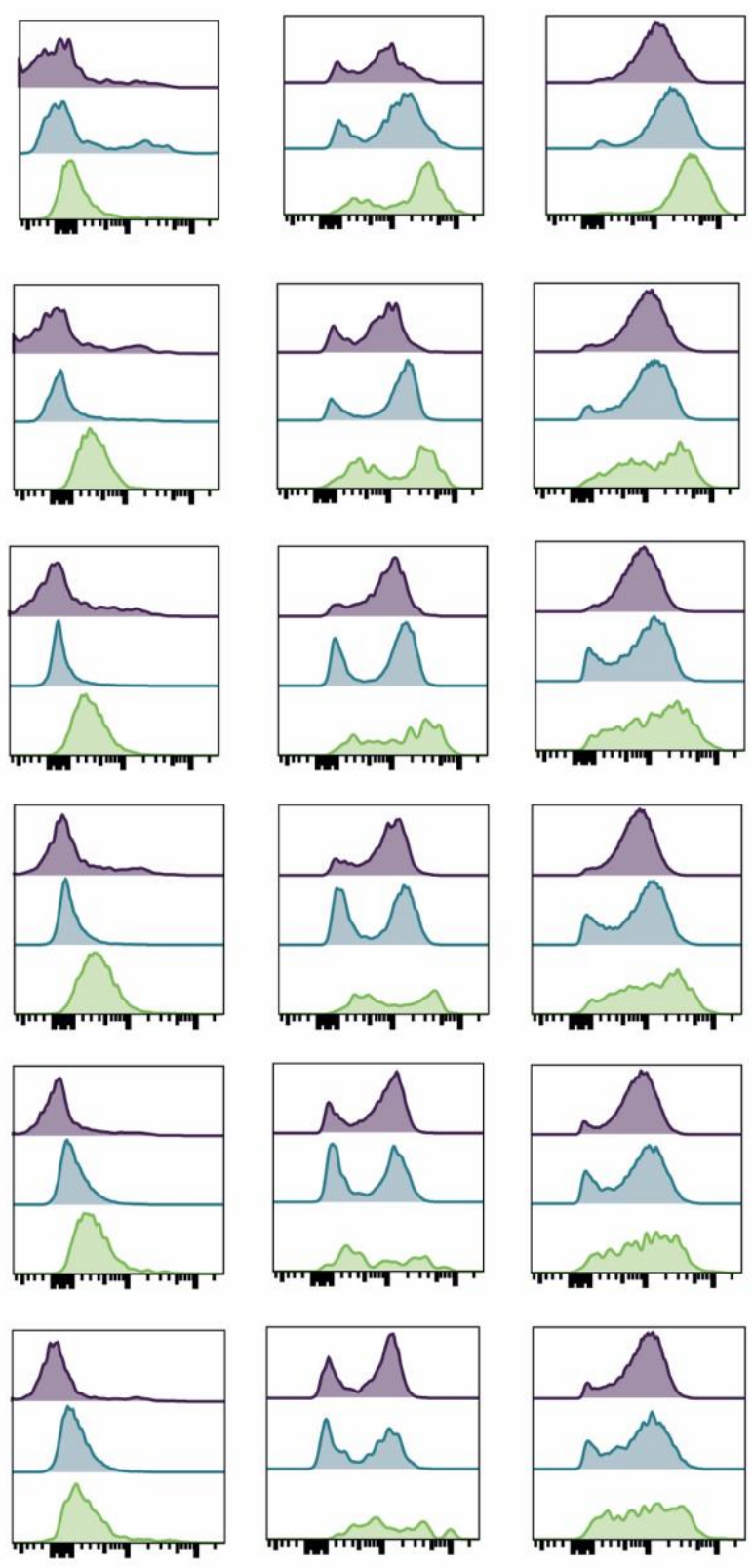

Mitoview Blue 


\subsubsection{HEL cells did not completely recover viability or mitochondrial membrane potential without co-culture after DFO treatment}

Previous results in chapter 4 show that co-culture of HL-60 0 o cells with un-injured HEL cells has the ability to increase the recovery from stress caused by deprivation of pyruvate and uridine. To assess any increase in viability from co-culture, a baseline recovery ability alone must be established. A recovery model designed to mimic the coculture conditions was used to test two concentrations of deferoxamine, $10 \mu \mathrm{M}$ and 25 $\mu \mathrm{M}$ (Fig 4.5.A). Cells were treated for 24 hours with the chosen concentrations.

Subsequently, media with drug was removed, cells were washed and fresh media placed to the treated cells. Cells were analysed for apoptosis and mitochondrial membrane potential 48 hours later.

Untreated HEL cells from this experiment exhibited slightly lower viability at the end point for this experiment than in the previous DFO titrations (Fig 4.4.B), around 80\% healthy cells, $5 \%$ early apoptotic, $5 \%$ late apoptotic cells and $10 \%$ cells in annexin negative cell death.

Forty-eight hours after the 24-hour $10 \mu \mathrm{M}$ deferoxamine treatment, 75\% of HEL cells were healthy, $10 \%$ of cells were split between early and late apoptosis and $15 \%$ of cells had undergone annexin negative cell death. Overall this indicates more non-apoptotic cell death in the HEL cells treated with $10 \mu \mathrm{M}$ DFO compared to the untreated samples.

Interestingly, HEL cells treated with $25 \mu \mathrm{M}$ DFO showed almost identical apoptotic populations and FSC SSC compared to the $10 \mu \mathrm{M}$ DFO treated cells (Fig 4.5.B).

Therefore, HEL cells were unable to fully recover from apoptois at either concentration of DFO treatment after 48 hours alone.

Mitoview blue analysis of mitochondrial membrane potential showed that both $10 \mu \mathrm{M}$ and $25 \mu \mathrm{M}$ deferoxamine treatment did not differ from untreated cells except in the early apoptotic populations. These cells had greater proportions of cells with low membrane potential (Fig 4.5.C). 


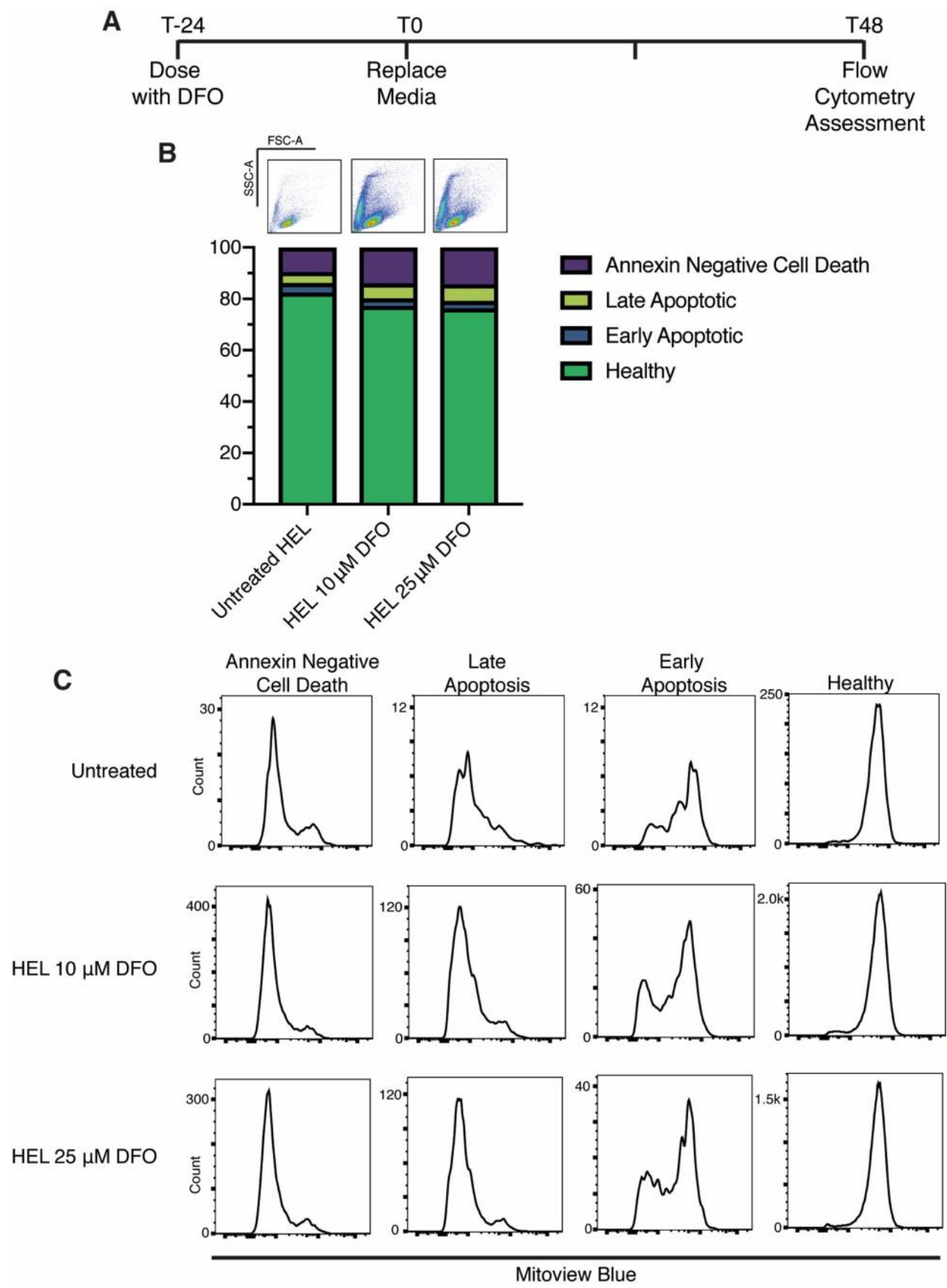

Figure 4.5: HEL cells did not completely recover viability or mitochondrial membrane potential without co-culture after 24 -hour $10 \mu \mathrm{M}$ or $25 \mu \mathrm{M}$ Deferoxamine treatment.

(A) Schematic representation of the recovery model of HEL cells treated with $10 \mu \mathrm{M}$ or 25 $\mu \mathrm{M}$ deferoxamine for 24 hours before replacement on media and removal of the drug. Cells were then left alone without co-culture for 48 hours and assessed for apoptosis and mitochondrial membrane potential using the gating strategy shown in Fig 4.3 and 4.4 (B) Graphical representation of apoptotic stages in untreated, $10 \mu \mathrm{M}$ and $25 \mu \mathrm{M}$ treated HEL cells. FSC and SSC plots are located above each treatment or assessment of morphology. (C) Histograms depicting Mitoview blue fluorescence for cells in each apoptotic population for untreated, $10 \mu \mathrm{M}$ or $25 \mu \mathrm{M}$ DFO treated HEL cells. Y scales are shown using 0 and the largest number for each graph for comparison. $(\mathrm{n}=1)$ 
The data shown here was collected from a single replicate, therefore specific effects could change if repeated again. While absolute conclusions cannot be made from this data, it can inform the suitability of concentrations for further co-culture experiments.

Alongside the apoptotic data it is possible to conclude that HEL cells transiently exposed to DFO cannot completely recover viability when alone in culture. The cells in early apoptotic phase may be the cells that could benefit from mitochondrial uptake from HL-60 cells in co-culture. Due to the lack of difference between $10 \mu \mathrm{M}$ and 25 $\mu \mathrm{M}$ DFO treatment, both concentrations were included in future co-culture experiments.

\subsubsection{Co-culture with HL-60 restored HEL viability after 24-hour deferoxamine treatment}

Once the baseline recovery alone was established, it was determined if co-culture with HL-60 cells could increase the viability of the HEL cells. Flow cytometry analysis was conducted by labelling each cell type with a different fluorescent dye (Fig 4.6.A). HEL cells were treated for 24 hours before addition into co-culture with fresh media. After treatment but prior to addition, HEL and HL-60 cells were stained using Viafluor 405 and Cell Trace Far Red respectively in preparation for FACS. Forty-eight hours later, co-cultured cells were separated. Two gating strategies were used for viability analysis, a strict PI negative gate, and a generous PI negative plus intermediate population (Fig 4.6.A). Intermediately stained cells may have the potential to reverse their permeability due to cell death further in culture 108,109. It was interesting to include them and assess the differences compared to solely negative populations.

Viability of HL-60 cells were assessed to ensure that co-culture did not impact on the viability of the HL-60 cell lines. These cells were not treated with any drug and therefore should not decrease in viability in any of the HEL treatment conditions. As expected, there was no difference between the viability of HL-60 cells cultured with HEL cells that were untreated, $10 \mu \mathrm{M}$ DFO or $25 \mu \mathrm{M}$ DFO treated. This trend was consistent between both strict and generous gating strategies with viability maintained above 95\% (Fig 4.6.B).

Using the strict PI negative gating strategy $10 \mu \mathrm{M}$ DFO treated HEL cell viability in coculture (mean of $87 \%$ ) was not significantly different to the recovery of treated HEL 
cells alone $(80 \%)$ or untreated co-culture $(91 \%)$ viability. Therefore, under strict gating strategies, the $10 \mu \mathrm{M}$ DFO did not show improved recovery compared to HEL cells cultured alone. However, the viability was not significantly different from the untreated control, indicating that the injury may not have been strong enough to cause detectable effect on viability.

HEL cell viability after $25 \mu \mathrm{M}$ DFO treatment (88\%) was significantly different to the recovery control $(80 \%, \mathrm{p} \leq 0.05)$ and these cells recovered to the same level as the untreated co-culture (91\%) (Fig 4.6.C). This demonstrated improved recovery from $25 \mu \mathrm{M}$ DFO treatment from co-culture.

Interestingly, when a generous gating strategy was used to assess viability the differences between samples changed (right graph). The average viability of HEL cells treated with $10 \mu \mathrm{M}$ DFO and then co-cultured with HL-60 increased from $87 \%$ to $92 \%$. This viability became significantly increased compared to the recovery control $(\mathrm{p} \leq 0.01)$, where the difference by only including PI negative cells was non-significant. The difference between untreated HEL cell viability and $10 \mu \mathrm{M}$ DFO treated HEL cell viability remained non-significant. Therefore, results indicate that co-culture did increase the viability of $10 \mu \mathrm{M}$ DFO treated HEL cells above recovery alone and to a similar level to untreated HEL cells.

After treatment with $25 \mu \mathrm{M}$ DFO and co-culture with HL-60, HEL cells recovered their average viability (as determined by PI negative gating) to $88 \%$. This was significantly higher than HEL cells treated with $25 \mu \mathrm{M}$ DFO and recovered alone $(\mathrm{p} \leq 0.05)$. Viability was not significantly different between untreated HEL cells and $25 \mu \mathrm{M}$ DFO treated HEL cells. The same results were seen when using both PI negative and intermediate cells ( $\mathrm{p} \leq 0.01$ ) (Fig 4.6.C). Therefore, HEL cells treated with $25 \mu \mathrm{M}$ DFO and cocultured with HL-60 cells were able to recover their viability above recovery alone and to a similar state as the untreated HEL cells.

Overall, co-culture with HL-60 cells rescued viability of HEL cells treated with both $10 \mu \mathrm{M}$ and $25 \mu \mathrm{M}$ DFO for 24 hours. 
A

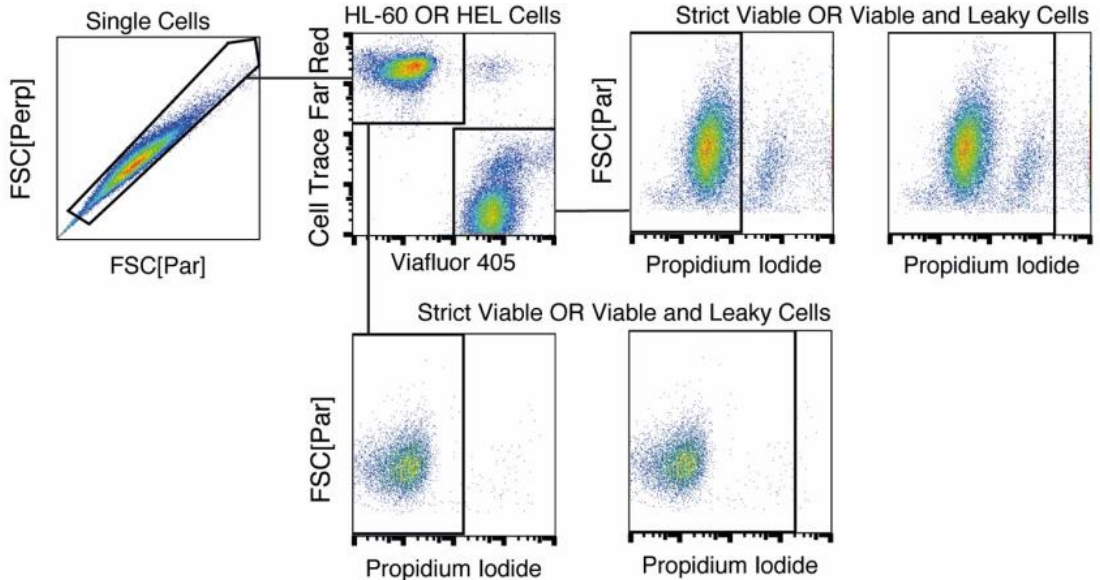

B
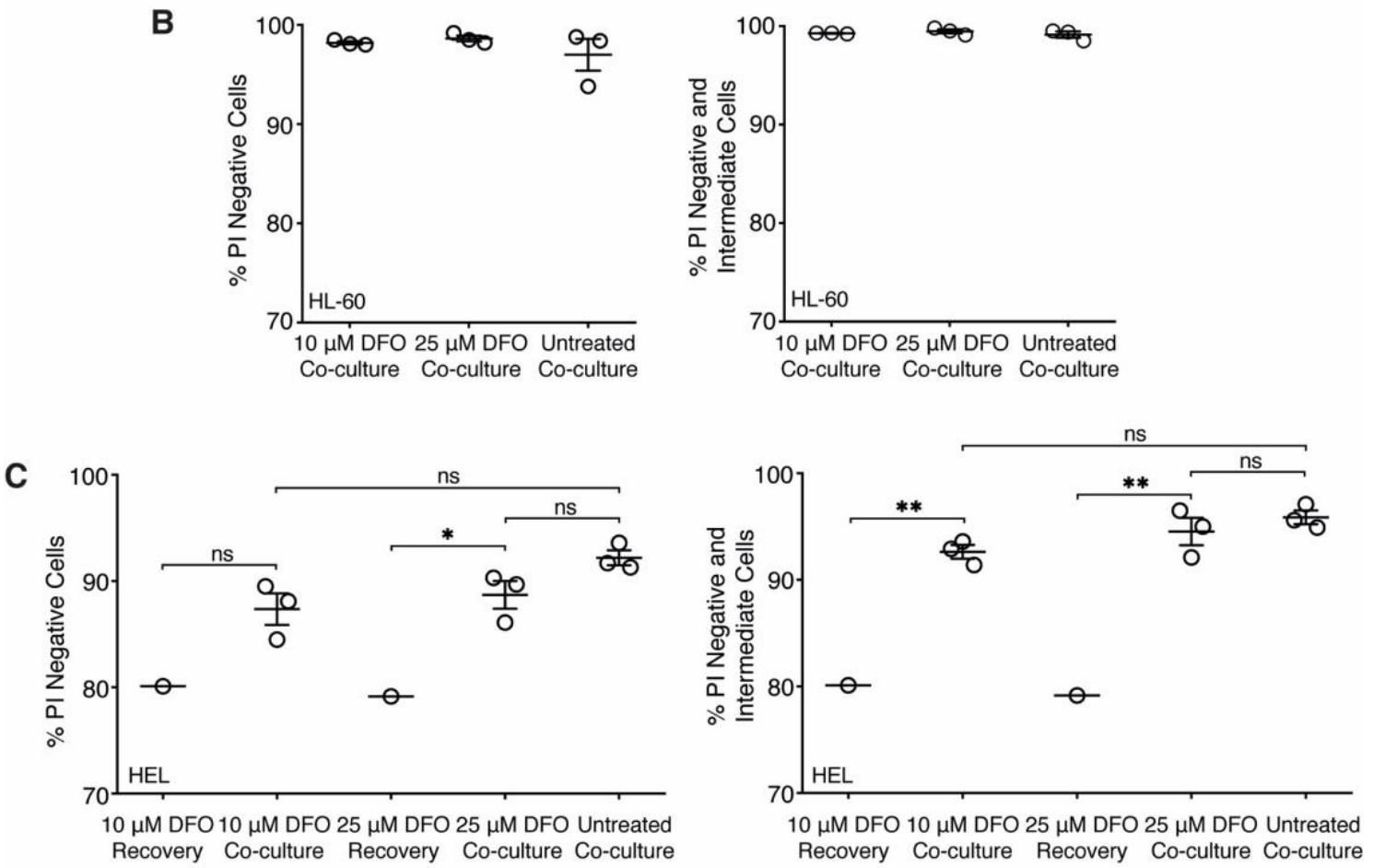

\section{Figure 4.6: Co-culture with HL-60 restored HEL viability after 24-hour deferoxamine treatment.}

(A) Flow cytometry gating strategy for viability analysis of HEL Viafluor 405 and HL-60 Cell Trace Far Red cells in co-culture using propidium iodide. Both strict PI negative and generous PI negative and intermediate gating was used. (B) Left graph shows strict PI negative gating to assess the viability of HL-60 cells at point of sorting that were untreated and co-cultured with untreated HEL, $10 \mu \mathrm{M}$ DFO or $25 \mu \mathrm{M}$ DFO treated HEL cells. Right graph shows the same conditions using the generous PI negative and intermediate gating strategy. (C) Left graph shows HEL viability using the strict PI negative gate for each co-cultured and sorted conditions. Zombie negative cell proportion from recovery experiments are also included for $10 \mu \mathrm{M}$ and $25 \mu \mathrm{M}$ DFO treated HEL cells as a comparative control for recovery without coculture. The right graph shows the same samples using the generous PI negative and intermediate gating strategy. Data on graphs are shown as mean \pm SEM. Statistical analysis was completed using One-Way ANOVA with Tukey's Post Hoc test using Prism 8 software. $\mathrm{ns}=$ no significance, ${ }^{*} \mathrm{p} \leq 0.05,{ }^{*} \mathrm{p} \leq 0.01 .(\mathrm{n}=3$ independent experiments $)$ 


\subsubsection{Mitochondrial DNA transfer did not occur to a detectable level between HL-60 and DFO treated HEL cells in co-culture}

To determine whether the enhanced recovery from DFO injury was correlated with transfer of mitochondria from healthy donor cells, DFO treated HEL cells, labelled with Viafluor 405 proliferation dye, were co-cultured with Cell Trace Far Red labelled untreated HL-60 cells. After 48 hours in co-culture, the two cell populations were separated using fluorescence associated cell sorting (FACS) and DNA was extracted from the populations for analysis via ASBqPCR.

The first step was to quantify the assay's ability to detect low amounts of transferred mtDNA. HL-60 DNA was titrated as a spike-in into HEL DNA, and the limit of detection calculated. The limit of detection for the HL-60 mtDNA Allele-specificblocker qPCR, when in a background of HEL DNA is $0.001 \%$ target mtDNA (Fig 4.7.A). To calculate the percentage of target DNA in a sample, the spike-in values were used to generate an equation based on a log-linear regression line fit to the data (Fig 4.7.B).

HL-60 cells were separated from untreated, $10 \mu \mathrm{M}$ DFO, or $25 \mu \mathrm{M}$ DFO treated HEL cells after co-culture via FACS and HL-60 mtDNA tested as a positive control to ensure mtDNA targets were reliably detected. There was no significant difference in the amplification cycle from HL-60 cells, between 17-18 cycles, regardless of the HEL cells they were co-cultured with (Fig 4.7.C).

The level of HL-60 mtDNA amplification in untreated HEL cells was taken as the baseline for analysis of the effect of mitochondrial transfer between treated samples. The HL-60 mtDNA signal from HEL samples from co-cultures amplified between 9-12 cycles earlier than the reference HEL DNA sample. The reference sample indicates the basal noise of the reaction created by a mis-match amplification of the off-target sequence. The HL-60 specific allele amplified significantly earlier from $10 \mu \mathrm{M}$ DFO treated HEL cells than the untreated co-culture $(\mathrm{p} \leq 0.001)$. However, in $25 \mu \mathrm{M}$ DFO treated HEL cells from co-culture the amplification was not significantly different from the untreated co-culture samples, likely due to the variation between the replicate experiments (Fig 4.7.C). 

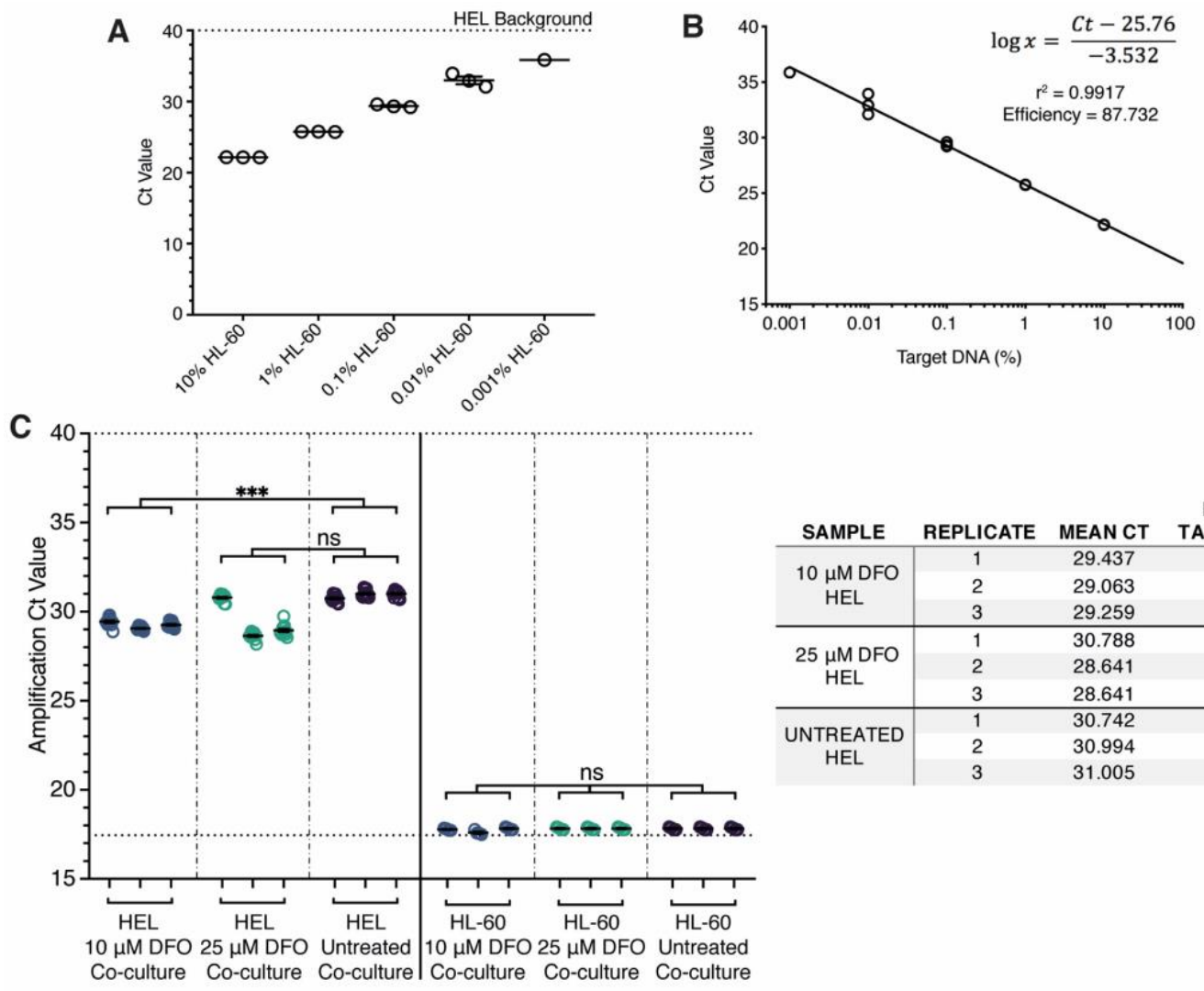

\begin{tabular}{c|ccc}
\multicolumn{1}{c}{ SAMPLE } & REPLICATE & MEAN CT & $\begin{array}{c}\text { MEAN } \% \\
\text { TARGET DNA }\end{array}$ \\
\hline $10 \mu \mathrm{M}$ DFO & 1 & 29.437 & 0.092 \\
$\mathrm{HEL}$ & 2 & 29.063 & 0.116 \\
& 3 & 29.259 & 0.103 \\
\hline \multirow{2}{*}{$25 \mu \mathrm{M}$ DFO } & 1 & 30.788 & 0.038 \\
$\mathrm{HELL}$ & 2 & 28.641 & 0.154 \\
& 3 & 28.641 & 0.127 \\
\hline UNTREATED & 1 & 30.742 & 0.039 \\
HEL & 2 & 30.994 & 0.033 \\
& 3 & 31.005 & 0.033
\end{tabular}

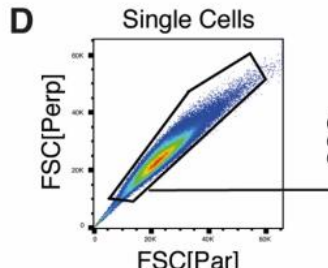

Cells of Interest

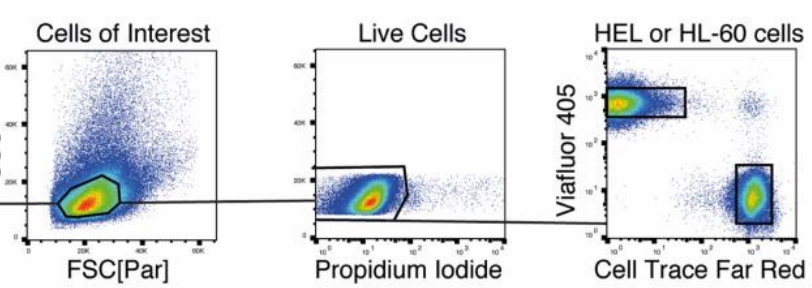

x2

E Cells of Interest

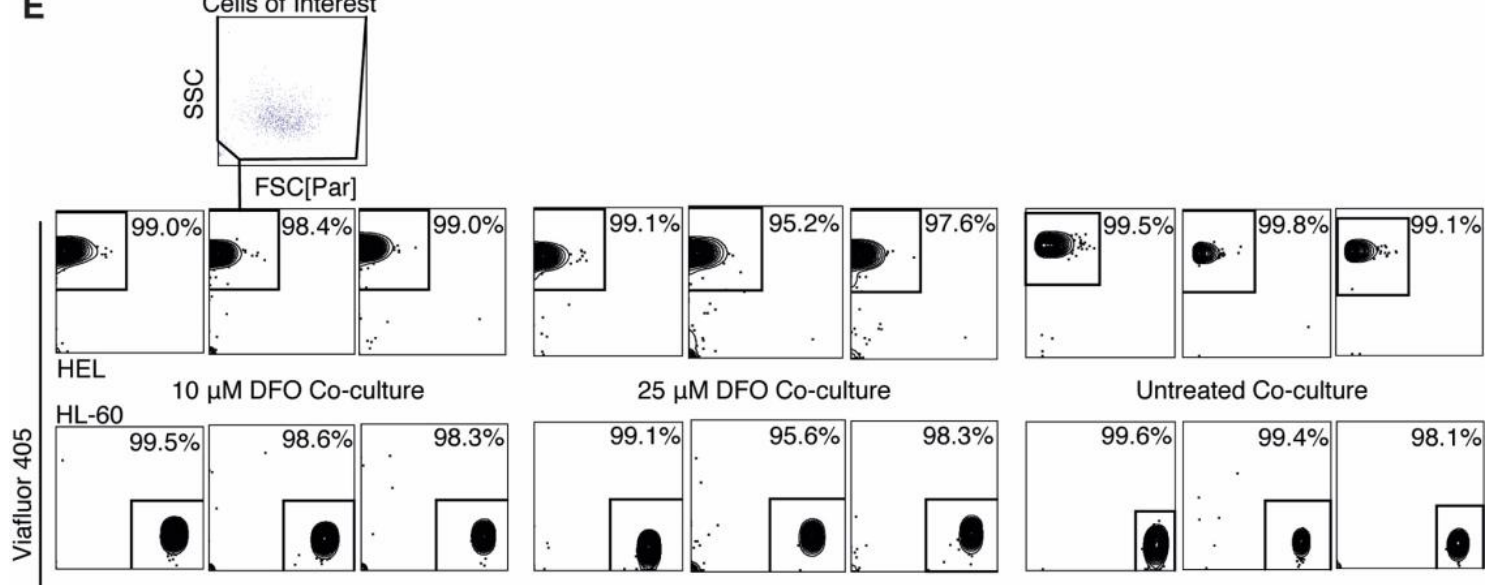

Cell Trace Far Red 


\section{Figure 4.7: Mitochondrial DNA transfer did not occur to a detectable level between HL-60 and deferoxamine treated HEL cells in co-culture.}

(A) Standard curve raw $\mathrm{Ct}$ points from an artificial spike in of between $10 \%-0.001 \%$ HL-60 DNA into a HEL background and a horizontal dotted line showing the limit of detection when $100 \%$ HEL background DNA is amplified on its own. (B) Standard curve correlating percent target DNA present to amplification $\mathrm{Ct}$ using the artificial spike-in $\mathrm{Ct}$ values. A log-linear line was fit to the data to create an equation to be used for calculating percent target DNA. The $\mathrm{r}_{2}$ value for this curve and the efficiency of the qPCR reaction are also provided. (C) Both HEL and HL-60 samples for untreated, $10 \mu \mathrm{M}$ and $25 \mu \mathrm{M}$ DFO treated (3 independent replicates each) were run through the ASBqPCR to detect HL-60 target mtDNA and their amplification $\mathrm{Ct}$ values are shown on the left. For each replicate 12 replicates wells of DNA were analysed for each HEL sample to detect mtDNA transfer and 4 replicate wells of DNA were tested for HL-60 as a control. Two horizontal lines show the limit of detection for a 100\% HEL background and the point at which 100\% HL-60 (positive control) amplified for reference. A nested t-test was conducted using Prism 8 software $* * * p \leq 0.001 \mathrm{~ns}=$ not significant. Data shows mean \pm SEM. To the left, tables show the mean ct amplification and mean calculated percent target HL-60 mtDNA in HEL samples that were untreated, $10 \mu \mathrm{M}$ and $25 \mu \mathrm{M}$ DFO treated and co-cultured. (D) Strategy used for sorting the HEL and HL-60 cells apart. Populations were double sorted through an initial enrich stage and a second one drop pure stage. (E) Plots showing purity of samples collected from the co-cultures for each condition. An example of the strategy used for purity assessment. Purity was assessed using a sample of 1000 cells. ( $n=3$ independent experiments) 
Using the equation from the standard curve, mean percent HL-60 target mtDNA was calculated for each replicate experiment. In $10 \mu \mathrm{M}$ DFO treated samples detected percentages of HL-60 mtDNA hovered around 0.1\% while HL-60 mtDNA present in $25 \mu \mathrm{M}$ DFO treated samples varied highly between $0.15 \%$ to $0.038 \%$. In comparison, the baseline HL-60 mtDNA amplification in untreated co-cultured HEL cells came up at $0.03 \%$, less than one third of HL- $60 \mathrm{mtDNA}$ present in $10 \mu \mathrm{M}$ DFO treated samples, and between no difference to one quarter less mtDNA present in $25 \mu \mathrm{M}$ DFO treated samples (Fig 4.7.D). From this data, it appeared possible that mtDNA transfer from HL60 into HEL cells may have occurred when HEL cells were treated with $10 \mu \mathrm{M}$ DFO for 24 hours. Unfortunately, the variance in amplification from $25 \mu \mathrm{M}$ DFO treated HEL cells prevented identification of mtDNA transfer in this condition.

However, this signal from HL60 mtDNA could also result from impure separation of the two cell types. To increase the purity obtained of HEL and HL-60 cells after coculture for DNA extraction, populations were double sorted. Firstly, populations were enriched, resulting in approximately $2 \%$ contamination and secondly, each enriched population was sorted using a one-drop pure phase (Fig 4.7.D). A small sample was taken from the final sorted populations before DNA extraction and qPCR analysis, for a 'cell purity' check. Purity was analysed by looking at a large 'cells of interest' gate followed by assessing proportions of proliferation dye stained cells (Fig 4.7.E).

Using this approach, the $10 \mu \mathrm{M}$ DFO treated HEL cell purity was between $98.4 \%$ and $99 \%$ for each replicate, while HL-60 cells were between $98.3 \%$ and $99.5 \%$ pure. Purity of the $25 \mu \mathrm{M}$ DFO treated HEL cells was between $95.2 \%$ and $99.1 \%$ while the HL-60 cells were between $95.6 \%$ and $99.1 \%$ pure. Untreated HEL cells displayed between $99.1 \%$ and $99.8 \%$ purity and HL-60 cells were between $98.1 \%$ and $99.6 \%$ pure. In all samples, $100 \%$ purity was not obtained, with contaminating cells present from either the opposing population, or from unstained cells where the origin could not be determined (Fig 4.7.E). Due to the extremely high sensitivity of the ASBqPCR assay for these cell lines, the $0.001 \%$ limit of detection is susceptible to false positives due to contamination. It is likely that in samples above $99.1 \%$ purity, for example the untreated HEL cells, the amplification of target HL-60 mtDNA at 0.03\% was due to low level contamination. Amplification appeared at earlier cycles in samples whose purity was below $99 \%$ such as those in the $10 \mu \mathrm{M}$ DFO co-culture and two replicates of the $25 \mu \mathrm{M}$ 
DFO co-culture. It is therefore highly likely that the significant difference in amplification detected in the ASBqPCR was due to increased contamination of HL-60 cells in the HEL DNA samples from the sort process.

\subsubsection{Cisplatin treatment induced morphological changes in K562 and reduced viability alongside mitochondrial membrane potential}

Deferoxamine was unable to induce detectable transfer of mitochondrial DNA in coculture conditions at the concentrations tested. An alternate drug that has been suggested to induce transfer of mitochondria is Cisplatin 57, a chemotherapy that links purine bases in DNA to interfere with replication and lead to cell death 51. The double stranded mitochondrial genome is affected by Cisplatin 51 and should therefore lead to a mitochondrial injury. The ability of cisplatin to induce cell death via apoptosis, and the level of injury to the mitochondria was assessed using an Annexin V / Zombie viability dye panel and Mitoview Blue stain, as performed in section 4.3.3 (Fig 4.8.A). K562 cells were treated with concentrations between $1 \mu \mathrm{M}-100 \mu \mathrm{M}$ Cisplatin over 96 hours in culture media. Cisplatin was dissolved in $0.9 \%$ sterile filtered $\mathrm{NaCl}$ solution and added at minimal amounts (less than $0.8 \mathrm{~mL}$ to $100 \mathrm{~mL}$ media), therefore a vehicle control was not included, rather cells were compared to an untreated control. Apoptotic stages were first analysed in the whole population of K562 cells present in the treated cultures, only excluding debris (Fig 4.8.B).

Untreated K562 cells maintained the expected lack of apoptosis associated with high viability and the characteristic FSC/SSC profile seen in earlier experiments. K562 cells treated with $1 \mu \mathrm{M}$ Cisplatin lacked significant induction of apoptotic populations but some increase in FSC/SSC was observed, suggesting that the drug had some effect on the cells, even at this low concentration.

Five and $10 \mu \mathrm{M}$ cisplatin treatment, at 24 and 48 hours, did not induce apoptosis compared to $1 \mu \mathrm{M}$ cisplatin treatment. However, at 96 hours, there was an induction of apoptosis, $10-20 \%$ early apoptotic cells and 15\% late apoptotic cells. Alongside the increases in apoptosis, the K562 cells doubled in size and granularity at 48 hours, and by 96 hours the cells had separated into two distinct populations of large, and very small, cells. 
Cells treated with $25 \mu \mathrm{M}$ cisplatin had 10\% early apoptosis at 24 hours. By 48 hours, $35 \%$ of cells were apoptotic ( $20 \%$ early, $15 \%$ late). At 96 hours of $25 \mu \mathrm{M}$ cisplatin treatment, approximately $10 \%$ of cells were early apoptotic while $50 \%$ of cells had entered late apoptosis. Notably, annexin negative cell death occurred in 10\% of K562 cells.

When K562 cells were treated with $50 \mu \mathrm{M}$ cisplatin, the proportion of healthy cells reduced by 24 hours, and $25 \%$ of cells were in early apoptosis. After 48 hours, numbers of healthy cells reduced further, $25 \%$ of cells were in early apoptosis and those in late apoptosis increased to $30 \%$. By 96 hours, only $15 \%$ of K562 cells treated with $50 \mu \mathrm{M}$ cisplatin remained healthy, $55 \%$ of cells were in late apoptosis while $20 \%$ of cells underwent annexin negative cell death.

One hundred $\mu \mathrm{M}$ cisplatin treatment of K562 cells resulted in $50 \%$ of cells remaining healthy at 24 hours and 35\% undergoing early apoptosis. By 48 and 96 hours, the proportion of cells that were in late apoptosis had increased to $50 \%$ and $60 \%$ respectively, with almost $20 \%$ annexin negative cell death at 96 hours.

The three lowest cisplatin concentrations $(1-10 \mu \mathrm{M})$ induced interesting changes to the FSC/SSC profile of K562 cells, not seen in the higher doses (25-100 $\mu \mathrm{M})$. These populations were gated, and apoptosis individually assessed to understand their response to cisplatin (Fig 4.8.C).

$1 \mu \mathrm{M}$ cisplatin treatment enlarged cells over 96 hours in culture, but no apoptosis was observed. Five $\mu \mathrm{M}$ cisplatin treatment induced larger cells at 48 hours which were less apoptotic than the total population (in Fig 4.8.B). By 96 hours almost 10\% of these large cells had undergone annexin negative cell death, which was not reflected in the overall population (Fig 4.8.B). A smaller cell population emerged at 96 hours which had more healthy cells than either the total population (Fig 4.8.B) or the large population. These small cells did not exhibit any annexin-negative cell death. This indicated the smaller cells were overall healthier than the large cells at 96 hours of $5 \mu \mathrm{M}$ cisplatin treatment.

The cells that enlarged from $10 \mu \mathrm{M}$ cisplatin treatment also maintained high proportions of healthy cells, around $90 \%$ over 48 to 96 hours. Again, the smaller cells were healthier 
than the total population, and similar to the large cells. At this dose there were no annexin-negative cells (Fig 4.8.C). It appears that low dose of cisplatin induced differentiation in K562 cells, and the smaller cells retained higher viability than enlarged cells.

To determine the extent of mitochondrial injury from cisplatin treatment in K562 cells, Mitoview blue stain was used to assess the level of mitochondrial membrane potential in each apoptotic stage, where reduced intensity of fluorescence correlates to reduced membrane potential. Untreated K562 cells displayed patterns of mitoview fluorescence consistent with other untreated cells (Fig 4.8.B).

Healthy cells exposed to increasing concentrations of cisplatin over 96 hours showed a concentration-dependent decrease in mito-membrane potential, indicating mitochondrial injury preceded cell death. Cells that entered early apoptosis showed a similar concentration-dependent decrease in fluorescence over 96 hours of treatment. The majority of late apoptotic cells initially had reduced mitochondrial membrane potential. With increasing cisplatin treatment, the minority of residual cells with mitochondrial membrane potential were lost over 96 hours. Treatment with increasing doses of cisplatin also corresponded to concentration-dependent reduction in mitochondrial membrane potential for cells that entered annexin-negative cell death (Fig 4.8.D).

Overall, cisplatin induced clear mitochondrial injury to membrane potential of K562 cells. These titrations were completed once, therefore can only provide an indication of the effect the concentrations have. Proportions of cell death may change if repeated. 
A

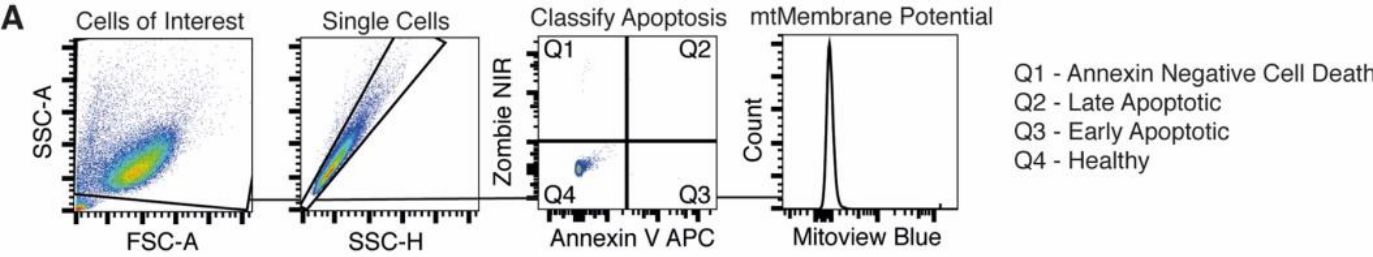

B
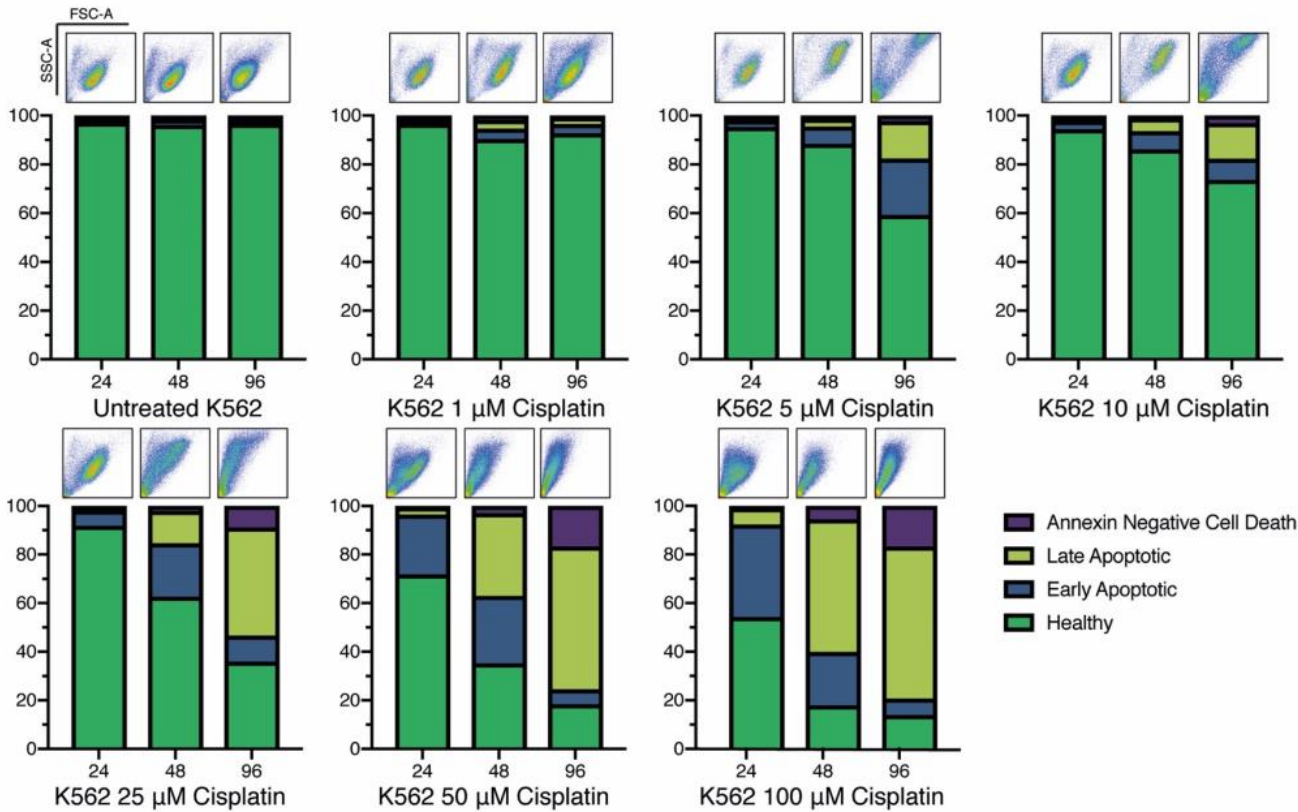

$\square$ Annexin Negative Cell Death

$\square$ Late Apoptotic

$\square$ Early Apoptotic

$\square$ Healthy

C

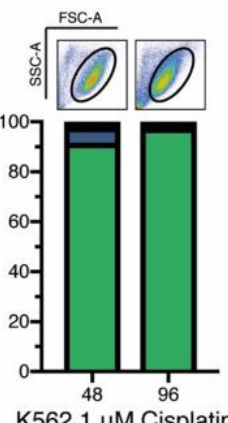

K562 $1 \mu$ M Cisplatin

Large Cells
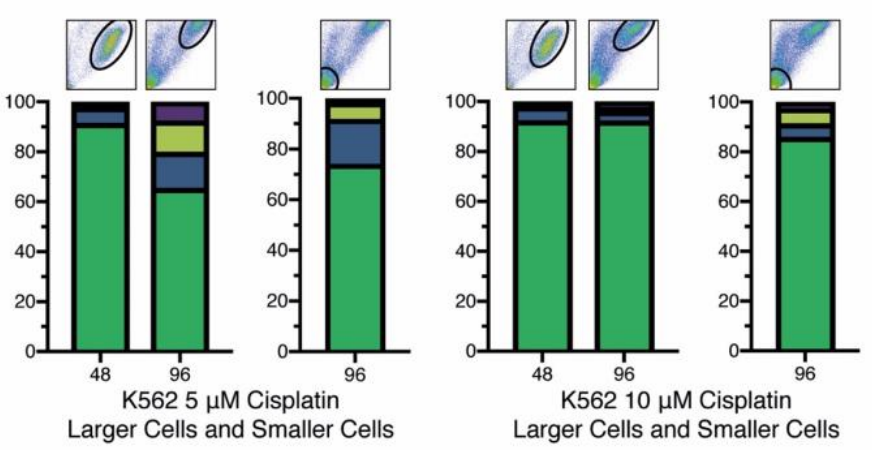

Figure 4.8: Cisplatin treatment induced morphological changes in K562 and reduced viability alongside mitochondrial membrane potential.

(A) Flow cytometry gating strategy using to identify apoptotic stages and the mitochondrial membrane potential of the cells within those stages. Same plots as shown in Fig 4.3. (B) Cisplatin titration graphs showing the four stages identified by an apoptosis panel: Annexin negative cells death, late apoptosis, early apoptosis and healthy (non-apoptotic). Above each column on each graph are FSC SSC plot showing morphology of cells at each time point measured. (C) Graphs identifying apoptotic populations present within specific FSC SSC populations present at 48 and 96 hours in three cisplatin concentrations $1 \mu \mathrm{M}, 5 \mu \mathrm{M}$ and $10 \mu \mathrm{M}$. (D) Histogram overlays of 24, 48 and 96-hour Mitoview blue fluorescence analysis for each apoptotic population of cells in each Cisplatin concentration. $(n=1)$ 
D
Untreated K562
K562 $1 \mu \mathrm{M}$ Cisplatin

Annexin Negative
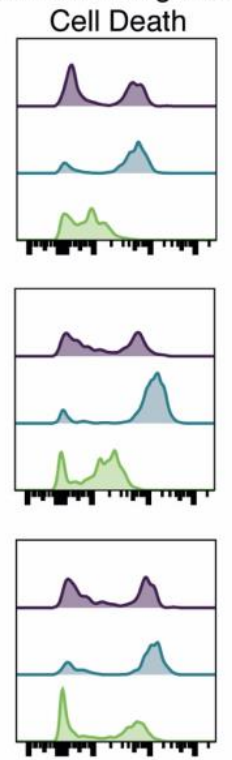

K562 $5 \mu \mathrm{M}$ Cisplatin

$$
\text { K562 } 5 \mu \text { M Cisplatin }
$$

K562 $10 \mu$ M Cisplatin
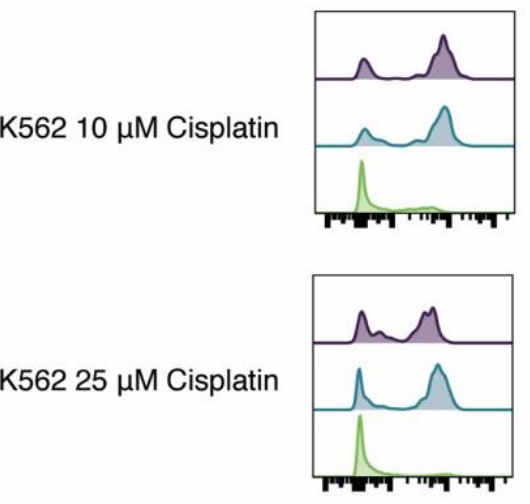

K562 $25 \mu \mathrm{M}$ Cisplatin
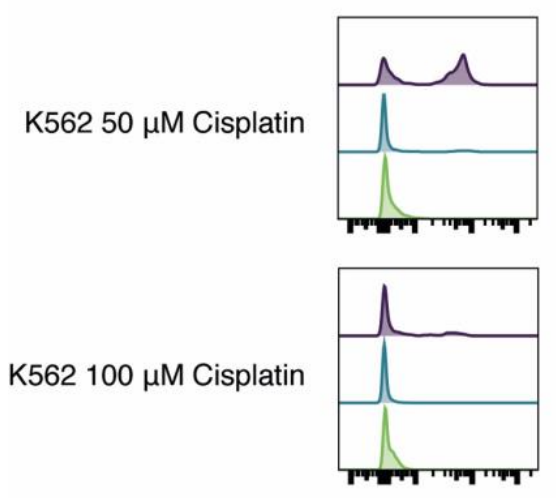
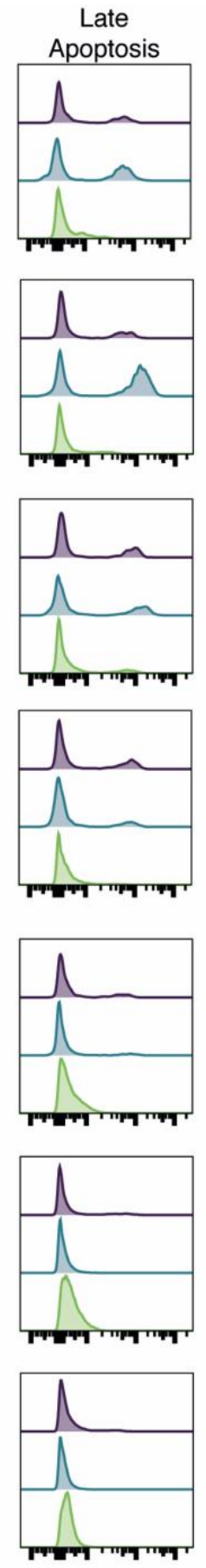

Early

Apoptosis
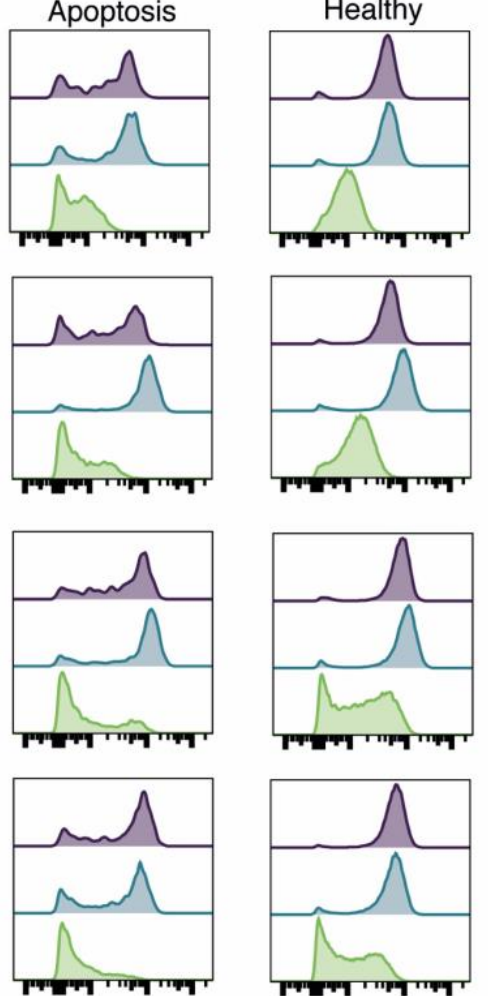

24 hours

$\square 48$ hours

96 hours
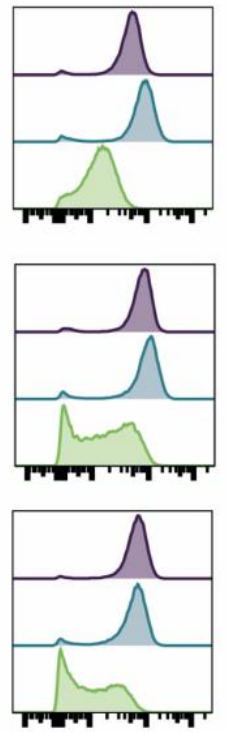
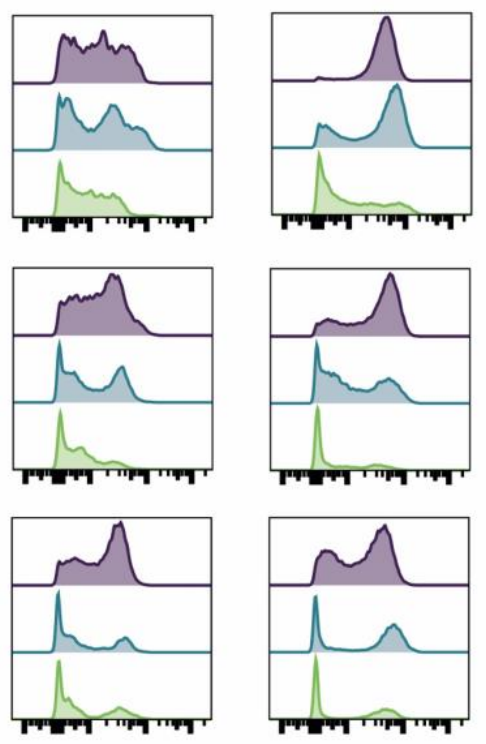

Mitoview Blue 


\subsubsection{HEL cells were highly sensitive to cisplatin treatment reduction in viability and mitochondrial membrane potential}

Cisplatin induced interesting effects in K562 cells, however, the large-scale changes in size made K562 cells unsuitable for further co-culture experiments. Sorting large cells increases the difficulty of obtaining the high purity samples required for these experiments due to fanning of droplet streams.

Cisplatin was titrated onto the HEL cells to identify a suitable dose for co-culture experiments (Fig 4.9.A). Analysis of cisplatin's effect on cell death and mitochondrial membrane potential was conducted the same way as with K562 cells (Fig 4.8).

Control untreated HEL cells were monitored at 24-, 48- and 96-hours. Untreated cells maintained the proportion of healthy cells around $90 \%$ with a tight FSC/SSC population consistent with previous data.

After 24 hours of $1 \mu \mathrm{M}$ cisplatin treatment, $10 \%$ of cells entered into early apoptosis and $5 \%$ of cells entered late apoptosis (Fig 4.9.B). Unexpectedly, at 48 hours $75 \%$ of cells stained for stages of cell death (50\% early apoptosis, $15 \%$ late apoptosis and $10 \%$ annexin-negative death). High level of cell death continued at 96 hours of treatment, where late apoptotic cells composed $70 \%$ of the population.

Early apoptotic cells increased to $35 \%$ at 24 hours when treated with $5 \mu \mathrm{M}$ cisplatin, and to $50 \%$ at 48 hours. By 96 hours, $80 \%$ of cells were late apoptotic and less than $5 \%$ of cells remained healthy.

With $10 \mu \mathrm{M}$ cisplatin, healthy HEL cells decrease rapidly at 24 hours with $50 \%$ of cells indicating apoptotic cell death (45\% early and 5\% late). At 48 hours, the late apoptotic population of cells increased to $30 \%$ and to a further $70 \%$ at 96 hours.

Treatment with $25 \mu \mathrm{M}$ cisplatin induced early apoptosis in almost $65 \%$ of HEL cells at 24 hours. By 48 hours, the percentage of healthy cells decreased to $10 \%$ with the remaining 90\% predominantly early and late apoptotic cells. At the 96-hour almost all cells entered a form of cell death (10\% early apoptosis, $80 \%$ late apoptosis and $10 \%$ annexin-negative cell death). 
HEL cells treated with $50 \mu \mathrm{M}$ and $100 \mu \mathrm{M}$ cisplatin treatment progressed similarly to $25 \mu \mathrm{M}$ cisplatin in apoptotic profiles. The only notable difference was the increase of cell in annexin negative cells death at 96 hours. At $25 \mu \mathrm{M}$ this was 5\%, which increased with $50 \mu \mathrm{M}$ to $10 \%$ and again with $100 \mu \mathrm{M}$ to $15 \%$.

The FSC/SSC over 96 hours did not change significantly with increased concentration of cisplatin (1-100 $\mu \mathrm{M})$. Cells increased in granularity (SSC) and decreased in size (FSC) over 96 hours regardless of their treatment (Fig 4.9.B).

Altogether, these results show that HEL cells were particularly susceptible to apoptosis induced by cisplatin treatment even at low concentrations. Interestingly, cisplatin treatment did not lead to induction of annexin negative cell death as seen in deferoxamine treatment of HEL cells. Additionally, HEL cell size was not impacted to the same degrees as K562 cells. All of these factors made HEL cells preferable for coculture experiments.

Next, the degree of injury to mitochondrial membrane potential cisplatin treatment induces in HEL cells was investigated. The control untreated HEL cells maintained consistent profiles in mitoview blue fluorescence correlating to mitochondrial membrane potential.

Cisplatin had a similar effect on HEL cell mitochondrial membrane potential (Fig 4.9.C) as on K562 cells (Fig 4.8.D). Even healthy cells that were exposed to increasing concentrations of cisplatin saw a concentration-dependent reduction in membrane potential, before cell death was induced. By 96 hours, increased number of cells in early apoptosis lost their membrane potential, correlated to increased concentration of cisplatin. Late apoptotic cells lost their residual population with mitochondrial membrane potential by 96 hours, as did cells that entered annexin negative cell death (Fig 4.9.C).

Overall, cisplatin treatment induced a strong injury to mitochondrial membrane potential in HEL cells that was concentration-dependent. Similarly, with K562 cisplatin titrations, these experiments were conducted a single time. However, the conclusions are based from the overall trend of each concentration tested. 

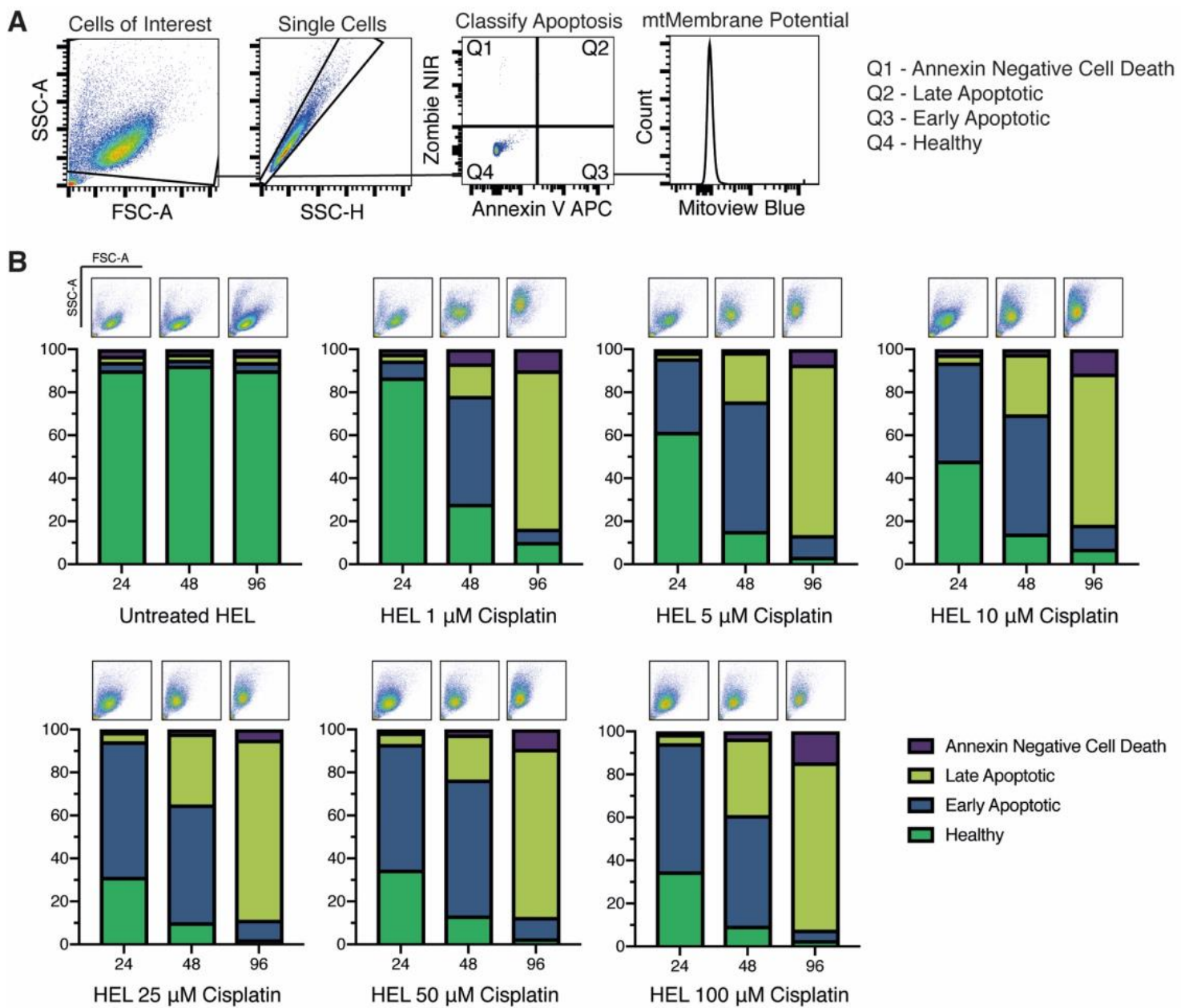

\section{Figure 4.9; HEL cells were highly sensitive to cisplatin treatment reduction in viability and mitochondrial membrane potential.}

(A) Flow cytometry gating strategy using to identify apoptotic stages and the mitochondrial membrane potential of the cells within those stages. Same plots as shown in Fig 4.3. (B) Cisplatin titration graphs showing the four stages identified by an apoptosis panel: Annexin negative cells death, late apoptosis, early apoptosis and healthy (non-apoptotic). Above each column on each graph are FSC SSC plot showing morphology of cells at each time point measured. (C) Histogram overlays of 24, 48 and 96-hour Mitoview blue fluorescence analysis for each apoptotic population of cells in each Cisplatin concentration. $(n=1)$ 


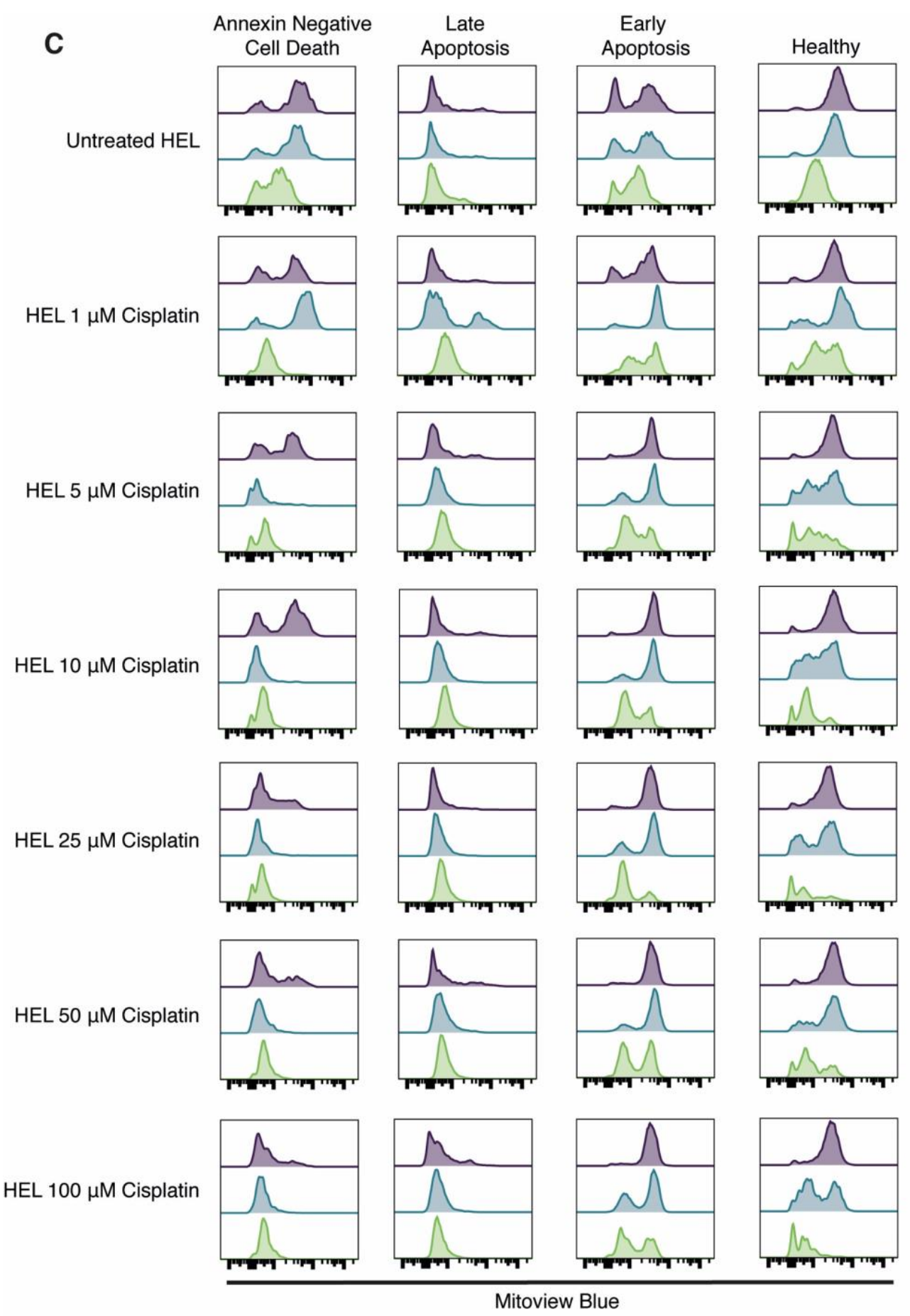




\subsubsection{HEL cells were unable to recover viability and mitochondrial membrane potential from 24-hour $1 \mu \mathrm{M}$ Cisplatin treatment without co-culture}

Similar to earlier deferoxamine work, a baseline level of recovery was identified. HEL cells were left either untreated, or treated for 24 hours with two candidate concentrations, $1 \mu \mathrm{M}$ or $5 \mu \mathrm{M}$ cisplatin. After 24 hours, cisplatin was removed, and fresh media added to all three conditions. Subsequently, cells were left for 48 hours before analysis of apoptotic populations and mitochondrial membrane potential (Fig 4.10.A).

Untreated HEL cells showed $90 \%$ of cells were healthy with minimal indication of apoptosis or annexin negative cell death. The FSC/SSC profile also indicated the cells were healthy. HEL cells that were treated with $1 \mu \mathrm{M}$ of cisplatin for 24 hours maintained around 50\% viability 48 hours later, and most of the remaining cells were in late apoptosis. The FSC/SSC of the $1 \mu \mathrm{M}$ treated cells was consistent with apoptosis.

Only $25-30 \%$ of cells treated with $5 \mu \mathrm{M}$ cisplatin remained healthy 48 hours after treatment. $10 \%$ of cells entered annexin negative cell death similar to the $1 \mu \mathrm{M}$ treated cells. Cells that entered early apoptosis increased to $20 \%$ and cells entering late apoptosis increased to $40 \%$. FSC SSC of the $5 \mu \mathrm{M}$ cisplatin treated cells did not differ from $1 \mu \mathrm{M}$ cisplatin treated HEL cells (Fig 4.10.B).

A similar assessment on mitochondrial membrane potential injury was conducted as per DFO recovery experiments. $5 \mu \mathrm{M}$ cisplatin led to a very clear sustained mitochondrial injury. Bimodal distribution appears in the apparently healthy cells, indicating that some cells sustained significant loss of membrane potential at $5 \mu \mathrm{M}$. This did not appear to the same extent in $1 \mu \mathrm{M}$ treated healthy cells and not at all in the untreated HEL cells. The same trend can be seen in the early apoptotic cells where $5 \mu \mathrm{M}$ cisplatin induced half of cells to lose fluorescence and this occurred to a lesser extent in $1 \mu \mathrm{M}$ cisplatin treated early apoptotic cells. In the late apoptotic cells, membrane potential did not change from either $1 \mu \mathrm{M}$ or $5 \mu \mathrm{M}$ cisplatin treatment. 


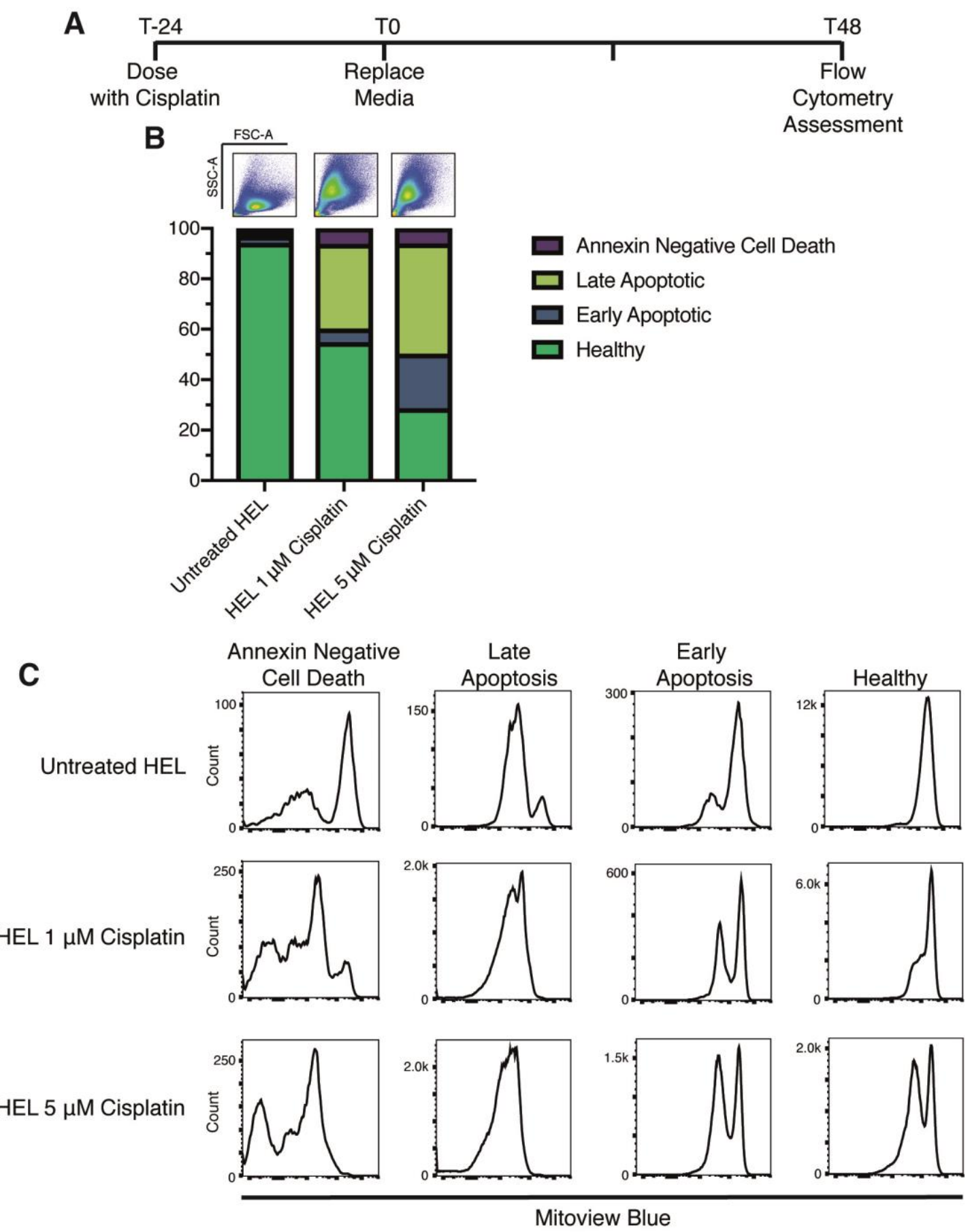

Figure 4.10: HEL cells were unable to recover viability and mitochondrial membrane potential from 24-hour $1 \mu \mathrm{M}$ Cisplatin treatment without co-culture.

(A) Schematic representation of the recovery model of HEL cells treated with $1 \mu \mathrm{M}$ or $5 \mu \mathrm{M}$ cisplatin for 24 hours before replacement on media and removal of the drug. Cells were then left alone without co-culture for 48 hours and assessed for apoptosis and mitochondrial membrane potential using the gating strategy shown in Fig 4.3 and 4.4 (B) Graphical representation of apoptotic stages in untreated, $1 \mu \mathrm{M}$ and $5 \mu \mathrm{M}$ treated HEL cells. FSC and SSC plots are located above each treatment or assessment of morphology. (C) Histograms depicting Mitoview blue fluorescence for cells in each apoptotic population for untreated, 1 $\mu \mathrm{M}$ or $5 \mu \mathrm{M}$ cisplatin treated HEL cells. Y scales are shown using 0 and the largest number for each graph for comparison. $(\mathrm{n}=1)$ 
Even though this particular experiment was completed once, the trends remain similar to the previous titrations (in Fig 4.9). Overall, the data showed that $5 \mu \mathrm{M}$ cisplatin treatment will be too severe for HEL cells to recover from, likely even with mitochondrial transfer. The number of cells entering late apoptosis was too high and the loss of mitochondrial membrane potential was extreme. Therefore, co-culture experiments proceeded with $1 \mu \mathrm{M}$ cisplatin treatment in attempts to induce mitochondrial transfer.

\subsubsection{Co-culture with HL-60 did not rescue cisplatin induced reduction in HEL viability}

Co-culture was able to abrogate the effects of DFO treatment on viability of HEL cells. Identical experiments were conducted to determine if co-culture also improved HEL viability when treated with $1 \mu \mathrm{M}$ cisplatin for 24 hours. The viability of HL-60 cells was measured, as a control for the health of the "donor" population and to ensure culture media would not reduce cell viability. Both 'strict' and' generous' PI gating strategies were used. (Fig 4.11.A). HL-60 cells maintained high viability regardless of culture with untreated HEL cells or HEL cells treated with $1 \mu \mathrm{M}$ cisplatin (Fig 4.11.B).

The difference in viability between untreated HEL cells and HEL cells that were treated with $1 \mu \mathrm{M}$ cisplatin and left alone to recover was around $40 \%$, providing a large window for co-culture to have an effect. However, HEL cells treated with $1 \mu \mathrm{M}$ cisplatin and co-cultured with HL-60 cells had significantly less viability, using the strict gating strategy, than both the recovery model $(\mathrm{p} \leq 0.01)$ and the untreated HEL cell control $(\mathrm{p} \leq 0.001)$. The viability for the $1 \mu \mathrm{M}$ cisplatin treated cells fell between 20 $30 \%$, less than half the viability of the recovery model (left graph Fig 4.11.C). However, using the gating strategy that includes PI intermediately stained cells as viable, after 24-hour $1 \mu \mathrm{M}$ cisplatin treatment and co-culture with HL-60 cells, HEL cells recovered to the same level as the recovery model where cells recovered alone. The differences between the two conditions were not significant, with high variation in the viability of the co-culture replicate experiments. The viability of the treated HEL cells in co-culture were significantly lower than the untreated co-culture control $(\mathrm{p} \leq 0.05)$ also indicating that co-culture did not increase viability. 
A
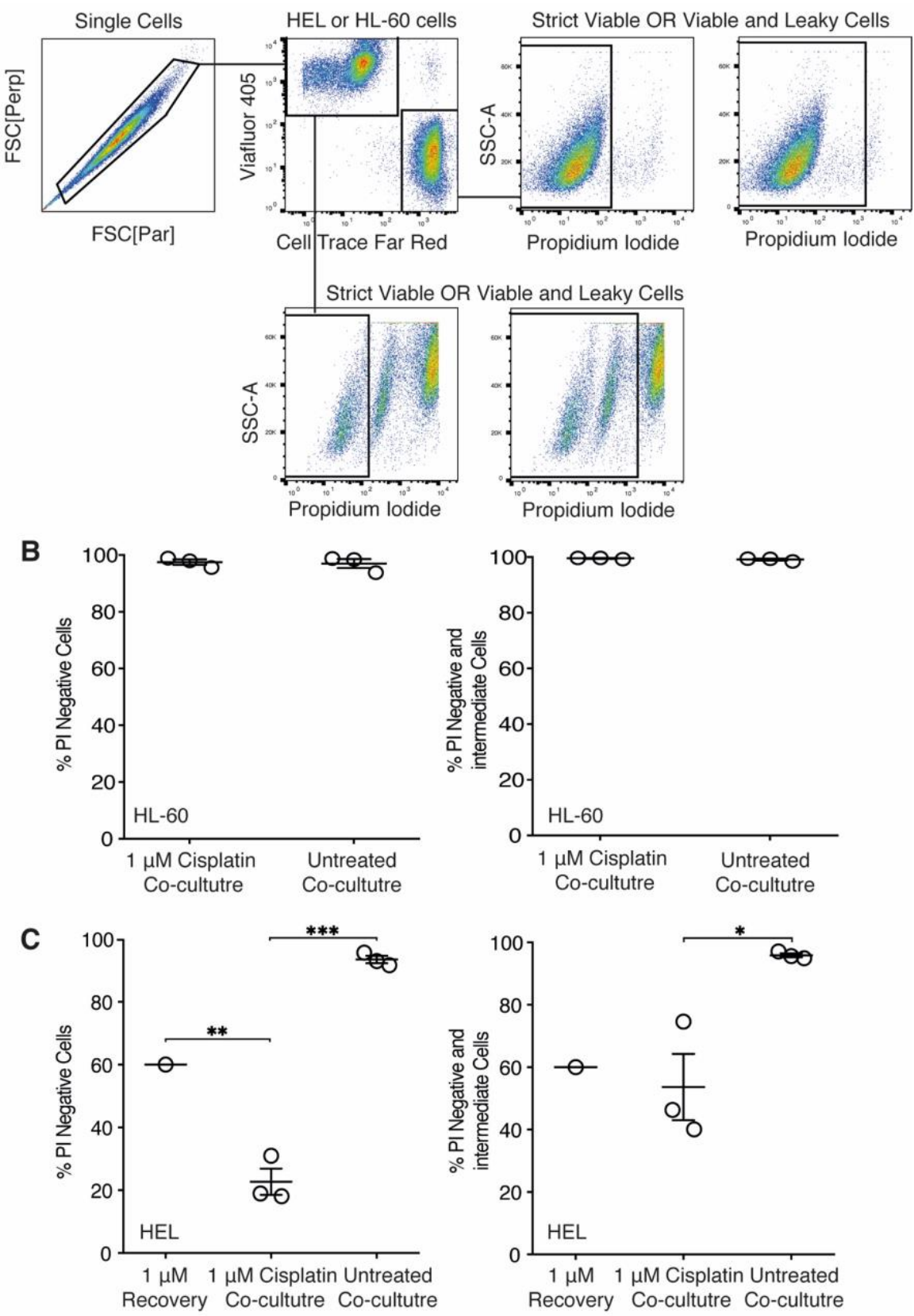

Figure 4.11: Co-culture with HL-60 did not rescue cisplatin induced reduction in HEL viability.

(A) Flow cytometry gating strategy for viability analysis of HEL Viafluor 405 and HL-60 Cell Trace Far Red cells in co-culture using propidium iodide. Both strict PI negative and generous PI negative and intermediate gating was used. (B) Left graph shows strict PI negative gating to assess the viability of HL-60 cells at point of sorting that were untreated and co-cultured with untreated or $1 \mu \mathrm{M}$ cisplatin treated HEL cells. Right graph shows the same conditions using the generous PI negative and intermediate gating strategy. (C) Left graph shows HEL viability using the strict PI negative gate for each co-cultured and sorted conditions. Zombie negative cell proportion from recovery experiments are also included for $1 \mu \mathrm{M}$ cisplatin treated HEL cells as a comparative control for recovery without co-culture. The right graph shows the same samples using the generous PI negative and intermediate gating strategy. Any data under the untreated co-culture category is the same as was used in Figure 4.6. Data on graphs are shown as mean \pm SEM. Statistical analysis was completed using One-Way ANOVA with Tukey's Post Hoc test using Prism 8 software $* \mathrm{p} \leq 0.05, * * \mathrm{p} \leq 0.01, * * * \mathrm{p} \leq 0.001$. ( $\mathrm{n}=3$ independent replicates) 
Overall, the cisplatin injury was still too severe for HEL cells to recover from with help from co-culture. Interestingly, strict PI gating suggested HEL cells in co-culture had even less ability to recover from the injury. This may be due to residual cisplatin carryover into the co-culture, or that cisplatin injury is more difficult to reverse as cells recover from apoptosis. Although it is not possible currently to fully determine the cause of this unexpected result.

\subsubsection{Transfer of HL-60 mitochondrial DNA did not occur to a detectable level into $1 \mu \mathrm{M}$ Cisplatin treated HEL cells}

The cisplatin co-cultures involved the same cell lines and looked for mtDNA transfer in the same direction as the deferoxamine treatments, HL-60 mtDNA into a HEL background. The limit of detection of this assay, calculated using the artificial spike in model, was therefore still $0.001 \%$ target HL-60 mtDNA (Fig 4.12.A). The regression equation used to calculate the percent of target HL-60 mtDNA was also the same as used previously (Fig 4.12.B). HEL cells were treated with $1 \mu \mathrm{M}$ cisplatin 24 hours prior to co-culture. HEL cells and HL-60 cells were stained with Viafluor 405 and Cell Trace Far Red respectively prior to co-culture in fresh media. Cells were separated 48 hours later using FACS, DNA was extracted and analysed using the HL-60 mtDNA specific ASBqPCR.

HL-60 cells that were co-cultured with HEL cells treated with $1 \mu \mathrm{M}$ cisplatin or untreated HEL cells amplified close to or at the same cycle as the 100\% HL-60 reference. As a result, there was also no significant difference between the mtDNA signal of the two groups of HL-60 cells. The HEL cells left untreated prior to co-culture maintained very tight amplification of the specific mtDNA allele between repeated experiments, at around 31 cycles. Conversely, the $1 \mu \mathrm{M}$ cisplatin treated repeated experiments displayed higher variation in amplification, between 30 and 32.5 cycles. The percent of HL-60 mtDNA present in untreated HEL cells after co-culture amplified was around $0.03 \%$ while the $1 \mu \mathrm{M}$ cisplatin treated HEL cells contained between $0.06 \%$ and $0.012 \%$ target $\mathrm{Hl}-60 \mathrm{mtDNA}$. Due to this high variation between the $1 \mu \mathrm{M}$ cisplatin treated repeats, there was no significant difference between untreated and $1 \mu \mathrm{M}$ cisplatin treated conditions. Both $1 \mu \mathrm{M}$ cisplatin treated, and untreated samples, amplified significantly higher than the 100\% HEL background reference. This could be 
an indication of transfer of HL-60 mtDNA in both conditions (Fig 4.12.C). Because there was no significant difference between the untreated and $1 \mu \mathrm{M}$ cisplatin treated conditions it was not clear if mtDNA transfer occurred due to cisplatin injury.

To distinguish if increased amplification above the $100 \%$ background reference was indicative of mtDNA transfer, the purity of samples was considered. As previously, to increase the sort purity for the cell lines, each population was initially sorted through an enrich stage and then through a one drop pure stage (Fig 4.12.D). Following the final purification, a small sample was taken to test 1000 cells for purity. $1 \mu \mathrm{M}$ cisplatin HEL and HL-60 co-culture samples were between $98.6 \%$ and $99.1 \%$, and $99.6 \%$ and $100 \%$ pure respectively. The untreated co-culture HEL cells had purity between $99.1 \%$ and 99.8\% and HL-60 cells had between $98.1 \%$ and $99.6 \%$ purity (Fig 4.12.E). Because the sensitivity of the ASBqPCR between these cells can detect up to $0.001 \%$ target mtDNA, the HEL populations need to be $100 \%$ pure to detect any mtDNA transfer occurring from co-culture. None of the HEL cell samples reached that purity level therefore the amplification results were likely skewed by contaminating cells. 

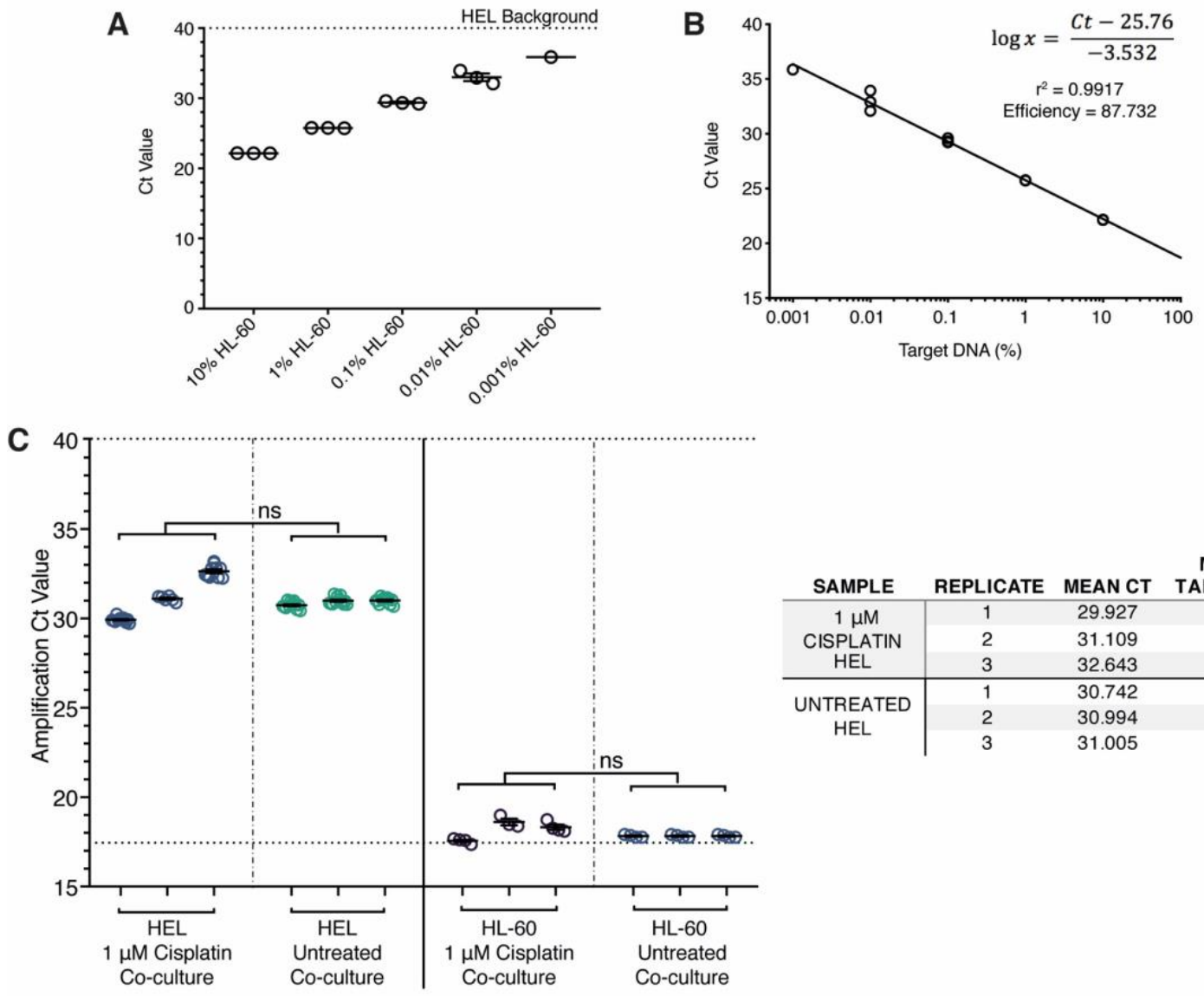

D

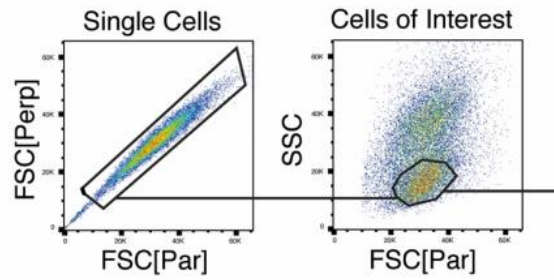

Live Cells

\begin{tabular}{c|ccc} 
SAMPLE & REPLICATE & MEAN CT & TARGET DNA \\
\hline $1 \mu \mathrm{M}$ & 1 & 29.927 & 0.066 \\
CISPLATIN & 2 & 31.109 & 0.031 \\
HEL & 3 & 32.643 & 0.012 \\
\hline UNTREATED & 1 & 30.742 & 0.039 \\
HEL & 2 & 30.994 & 0.033 \\
& 3 & 31.005 & 0.033
\end{tabular}

E

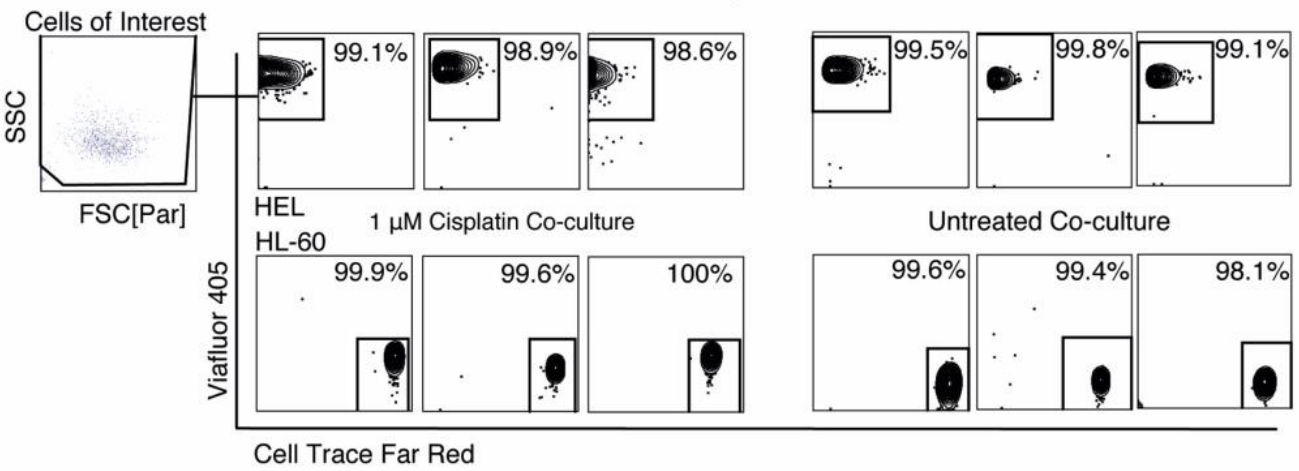


Figure 4.12: Transfer of HL-60 mitochondrial DNA did not occur to a detectable level into $1 \mu \mathrm{M}$ Cisplatin treated HEL cells.

(A) Standard curve raw $\mathrm{Ct}$ points from an artificial spike in of between $10 \%-0.001 \%$ HL-60 DNA into a HEL background and a horizontal dotted line showing the limit of detection when 100\% HEL background DNA is amplified on its own. (B) Standard curve correlating percent target DNA present to amplification $\mathrm{Ct}$ using the artificial spike-in Ct values. A log-linear line was fit to the data to create an equation to be used for calculating percent target DNA. The $\mathrm{r}_{2}$ value for this curve and the efficiency of the qPCR reaction are also provided. (A\&B) show the same standard curve used in Fig 4.7. (C) Both HEL and HL-60 samples for untreated or $1 \mu \mathrm{M}$ cisplatin treated (3 independent replicates each) were tested using the ASBqPCR to detect HL-60 target mtDNA and their amplification Ct values are shown on the left. For each replicate 12 replicates wells of DNA were analysed for each HEL sample to detect mtDNA transfer and 4 replicate wells of DNA were tested for HL-60 as a control. Two horizontal lines show the limit of detection for a $100 \%$ HEL background and the point at which 100\% HL-60 (positive control) amplified for reference. A nested t-test was conducted using Prism 8 software $* * * p \leq 0.001 \mathrm{~ns}=$ not significant. Data shows mean \pm SEM. To the left, tables show the mean $\mathrm{Ct}$ amplification and mean calculated percent target HL-60 mtDNA in HEL samples that were untreated or $1 \mu \mathrm{M}$ cisplatin treated and co-cultured. (D) Strategy used for sorting the HEL and HL-60 cells apart. Populations were double sorted through an initial enrich stage and a second one drop pure stage. (E) Plots showing purity of samples collected from the co-cultures for each condition. An example of the strategy used for purity assessment. Purity was assessed using a sample of 1000 cells. Untreated samples qPCR data is the same as used in the deferoxamine data (Fig 4.7). $(\mathrm{n}=3)$ 


\subsection{Discussion}

Inter-erythroid mitochondrial transfer has not been investigated in research previously, therefore this thesis used model erythroid cell lines to explore the phenomenon. HEL cells were overall more sensitive to both deferoxamine and cisplatin treatment and were chosen for co-culture experiments over K562 cells. $10 \mu \mathrm{M}$ and $25 \mu \mathrm{M}$ deferoxamine injury to HEL cells was not sufficient in inducing detectable mitochondrial transfer from HL-60 cells. However, co-culture with HL-60 cells did promote increased viability recovery in HEL cells. Similarly, $1 \mu \mathrm{M}$ cisplatin injury to HEL cells was unable to induce mitochondrial transfer from HL-60 cells at a level higher than the limitation of the assay. The cisplatin injury appeared to be too severe for co-culture to improve survival of the HEL cells.

Part of the rationale for exploring mitochondrial transfer in erythroblasts was the expression of CD38 on the cell surface in the bone marrow79,80. CD38 is most commonly expressed at early stage erythroid differentiation by CD34+ progenitors of myeloid lineage and by pro-erythroblast cells79. Further, CD38 has very recently been implicated in the formation of tunnelling nanotubules, one of the most supported methods of transferring mitochondria between CD38 expressing multiple myeloma and non-CD38 expressing mesenchymal stem cells 63. Results showed that neither HEL and K562 cells lines expressed any CD38, while HL-60 cells and their po derivatives did (Fig 4.1). Interestingly, HEL and K562 are more erythroid like than the HL-60 cells. HEL was derived from a human erythroleukemia, while K562 is a chronic myeloid leukemia that can be differentiated to express embryonic and foetal haemoglobin83,110. HL-60 cells are from a promyelocytic lineage 84,86 . These results may indicate that HL60 cells are less differentiated myeloid cells, supported by the fact that HL-60 can differentiate into granulocytes and macrophages $43,84,86$. Similarly, lack of CD38 expression seen on K562 and HEL cells suggests they are further differentiated through the erythroid lineage than HL-60 cells. The role of CD38 in transfer of mitochondria is a new development and should be explored in future studies involved in this field.

Erythroblasts require iron to generate heme for haemoglobin used to bind oxygen when in their final erythrocyte stages34. Perl's stain can identify iron deposits and was used to investigate the presence of iron in the bone marrow erythroid cell lines HEL, K562 and HL-60 cell lines 88. Results showed that only HEL displayed any indications of iron 
deposits (Fig 4.1), at very low levels compared to that in bone marrow. This positive staining suggested iron may have a role in HEL cells, and this could indicate a response to deferoxamine treatment, due to the iron chelation effect. Low iron levels can lead to reduced erythroblast differentiation and survival 74, supporting iron loss as a cellular and mitochondrial injury.

Titrations of deferoxamine concentrations for both HEL and K562 induced two very distinct responses. Firstly, K562 cells were resistant to both apoptotic induction and mitochondrial membrane potential loss at low concentrations, $10 \mu \mathrm{M}$ and $25 \mu \mathrm{M}$ (Fig 4.3). Induction of apoptosis ensued at greater concentrations of deferoxamine including and above $50 \mu \mathrm{M}$ with both early and late apoptotic populations appearing. Induction of apoptosis was also modelled by the shifts in morphology for the cells. Previous research shows that cells in apoptotic stages change their morphology and can be visualised using basic non-fluorescent flow cytometry3. Characteristically, cells that undergo apoptosis reduce their FSC and increase their SSC causing a 'left' shift left from the healthy control populations 3. Late apoptotic cells decrease their SSC along with FSC shifting further to the left and down the SSC axis3. FSC/SSC analysis of K562 cells identified as apoptotic mirrored this phenotype. Additionally, notable changes to mitochondrial membrane potential were only present at high concentrations of deferoxamine, above $100 \mu \mathrm{M}$. This may indicate that early induction of apoptosis by DFO in K562 cells may only involve the extrinsic pathway, while apoptosis induced at later time points of treatment may be caused more by the intrinsic mitochondrial associated pathway of cytochrome c release3.

HEL cells responded very differently to deferoxamine treatment than K562 cells. While deferoxamine did induce some levels of apoptosis particularly by 48 hours, the major proportion of cell death came from cells that stained positive for Zombie viability but negative for Annexin V (Fig 4.4). These cells therefore died via pathways distinct from apoptosis. Several pathways of cell death fall under this annexin negative characterisation such as necrosis, necroptosis, pyroptosis, autophagic cell death and caspase-independent cell death 111. Necrosis is a process of cell death that is not controlled often resulting in cell swelling and vacuole formation in the cytoplasm 112. These changes can lead to an increase in cell FSC, cell size, and SSC, cell granularity112. HEL cells treated with deferoxamine at any concentration did not 
increase in their FSC, only SSC. A likely pathway that could explain the marked increase in SSC paired with a decrease in FSC seen in HEL cells is autophagic cell death111. During autophagy, autophagosomes are created in the cytoplasm of cells and used to degrade components of the cells111. These autophagosomes could increase the granularity of the HEL cells while their cell size would decrease as the degradation process continued. Deferoxamine treatment of HEL cells induced a reduction in mitochondrial membrane potential, in both apoptotic cells and cells undergoing annexin negative cell death. Loss of mitochondrial membrane potential in apoptotic cells may indicate intrinsic mitochondrial associated apoptotic mechanisms, as with K562 cells3. HEL cells undergoing non-apoptotic cell death may be autophagic, indicating that loss of membrane potential seen in cells could be due to degradation of mitochondria via autophagy, mitophagy. The reduced mitochondrial mass would also reduce total fluorescence of Mitoview blue111. It is very interesting how treatment from one compound can induce such different responses in these two cell lines.

Differences in cell line responses to drug treatments were also seen when HEL and K562 cells were treated with Cisplatin, a DNA intercalating chemotherapeutic113. K562 was resistant to induction of apoptosis at lower concentration of cisplatin, similarly to deferoxamine treatment. Interestingly, at concentrations of cisplatin treatment below $25 \mu \mathrm{M}$, resistance was associated with enlarged FSC/SSC. At 96 hours, a small cell emerged with greater viability than the larger counterparts (Fig 4.8). Previous published data shows that treating cells with concentrations lower than $50 \mu \mathrm{M}$ cisplatin induced differentiation of K562 into erythroid cells, that express haemoglobin whilst maintaining maximal viability 113,114 . The smaller cells present at lower cisplatin concentrations in these cultures may be the erythroid cells differentiating, but without further analysis using erythroid markers I cannot be sure this is the case. Cisplatin treatment also induced a large loss of mitochondrial membrane potential over long-term treatment of K562 cells, however, rapid loss at 24 hours was only seen at the highest dose tested, $100 \mu \mathrm{M}$ (Fig 4.8).

Comparatively, HEL cells were more sensitive to cisplatin treatment with large inductions of apoptosis early and at even the lowest concentration tested, $1 \mu \mathrm{M}$. FSC SSC shifts in HEL cells resemble that expected from previous research of classical apoptosis. Additionally, a maximal reduction in mitochondrial membrane potential was 
seen at $25 \mu \mathrm{M}$ cisplatin and higher concentrations (Fig 4.9). Opposite to deferoxamine treatment, sensitivity to cisplatin was not paired with a large increase in cells that underwent annexin negative cell death. Rather the effect still maintained the classical apoptotic phenotype. While deferoxamine chelates extracellular iron leading to relatively uncontrolled cellular effects, cisplatin intercalates with DNA leading to DNA damage via breakages inside the cell. The DNA damage response is known to induce apoptosis, thus a far more studied and controlled pathway of action than iron loss 49-51.

A recent influx of published data provides evidence that co-culture with healthy cells increases the outcome of viability for injured cells115-118. Several of these examples show support provided by fibroblasts 115,116 or mesenchymal stem cells117,118. The results of this thesis showed that when HEL cells were treated with deferoxamine before coculture, presence of HL-60 cells improved their viability at 48 hours, almost identical to the untreated control (Fig 4.6). The results in this chapter support this growing body of research, however these data also promote the idea that not only mesenchymal cells or similar are able to provide this assistance but also cells of similar type in proximity not effected by the injury. Cisplatin injury was too severe to see an effect of co-culture on HEL cell recovery (Fig 4.11), indicating a limit or effectiveness for co-culture support.

Mitochondrial DNA polymorphisms were identified between the HL-60, K562 and HEL cell lines for use in the allele-specific-blocker qPCR assay to detect mitochondrial transfer (Fig 4.2). These polymorphisms, m.150 $\mathrm{T}>\mathrm{C}$ and $\mathrm{m} .152 \mathrm{C}>\mathrm{T}$, were associated with known haplotypes of the human genome, J2a1a1a2119. The design of the assay allowed detection as low as $0.001 \%$ HL-60 target mtDNA when in a background of HEL DNA (Fig 4.2, 4.7 \& 4.12). However, similar to detecting HEL mtDNA in HL60 o cells after co-culture, contamination from using fluorescence associated cell sorting to separate populations lead to the inability to confidently detect mitochondrial DNA transfer into the treated HEL cells from HL-60 cells (Fig 4.7\&4.12).

A further way to determine the level of contamination in the DNA samples is to use a nuclear control to detect contaminating cells via presence of nuclear DNA. Throughout this thesis, short tandem repeats (STR) and Y chromosome PCRs were explored. However, STRs could not provide sensitivity required for the level of contamination for sorts and male HEL cells had not retained their Y chromosome via XY FISH detection 
(data not shown). Due to time constraints nuclear controls were unable to be developed for these co-cultures but should be done in future.

To try and improve the purity of the sort, the gating strategy used for the 'cell of interest' gates were kept very stringent, resulting in small yields. This approach could have skewed the data, by excluding cells that were not consistent with the morphology of the untreated cells. These unusual cells may have been where the mitochondrial transfer events occurred. However, this was necessary to achieve the highest possible purity. There are other techniques that could be used to analyse transfer of mitochondria whilst cells are still in co-culture, avoiding the issues around cell sorting. One of these, rolling circle amplification70 can detect allele-specific mtDNA molecules in situ, and has the potential to alleviate these issues.

Additionally, mtDNA transferred at 48 hours is likely at low levels and more difficult to detect using these detection methods. Cultures could therefore be left for longer periods to allow mtDNA establishment. Interestingly, our lab group has identified that ratios of donor to recipient cells can also influence occurrence of mitochondrial transfer in coculture and may be worth considering in future experiments.

Finally, these data provided cannot exclude the conclusion that mitochondrial transfer did occur. It is still not known if cells of the same origin are able to transfer to each other, let alone within the condition of erythroblasts.

\subsection{Conclusion}

While co-culture of injured HEL cells with HL60 cells improved their recovery from injury, it could not be shown that this was associated with mitochondrial transfer. However, these data do not prove that mitochondrial transfer does not happen between these cell types. In order to circumvent the problems associated with cell sorting, the two HL60 mtDNA SNP can be detected with another assay. Target-primed rolling circle amplification uses an allele-specific primer that essentially performs a PCR directly on fixed cells attached to a microscope slide, and a fluorescent probe to visualise the PCR product. 
5 Investigation of mitochondrial transfer between primary human erythroblasts ex vivo 


\subsection{Introduction}

Erythroblasts are red cell progenitors located in bone marrow, where they carry out an asymmetrical division process called erythropoiesis to differentiate into erythrocytes, which migrate to the peripheral blood stream 73 . Erythroblasts are originally derived from $\mathrm{CD} 34+$ common myeloid progenitors and differentiate through pro-erythroblast, basophilic, polychromatophilic and orthochromatophillic stages before a final extrusion of their mitochondria and nucleus allowing them to become mature red blood cells 73 . Mitochondria are heavily involved in iron metabolism and heme synthesis within these erythroblasts. Heme is able to direct gene expression, by binding to heme regulatory motifs found on some nuclear receptors, to produce haemoglobin used by erythrocytes to carry oxygen $35,120,121$. Additionally, heme is involved in formation and function of mitochondrial complexes I-IV 45. While erythroblasts can be modelled in vitro using cell line such as HEL, K562 and HL-60, as seen in this thesis, translation from these cell lines to human in vivo work is limited. To study human erythroblasts directly requires a clinical procedure called bone marrow aspiration. This is not a painless procedure, and is not often performed in healthy donors. Instead, most bone marrow aspirates are taken from patients who have cancer or other health conditions involving bone marrow 88. Erythroblasts obtained from these patients present with variables unable to be controlled such as disease, prior treatments and stressors which could impact on their use in mitochondrial transfer experiments.

Interestingly, a protocol has been published that generated human erythroblasts from adult peripheral blood mononuclear cells 87. Using components including stem cell factor (SCF), interleukin-3 (IL-3), erythropoietin (EPO), $\beta$-estradiol and dexamethasone up to $90 \%$ of the PBMC culture phenotypically resembled erythroblasts by day 17 of culture 87. Additionally, the method can be used to induce erythrocyte differentiation using EPO and insulin 87. This methodology provided a more readily accessible and ethical alternative to erythroblasts from bone marrow for use in this project to investigate mitochondrial transfer.

Mitochondrial transfer within the bone marrow has been studied to a small extent - three bodies of data have been published in the last 5 years. The first paper explored transfer of mitochondria from human and mouse bone marrow stromal cells into human acute myeloid leukemia cells (AML), and showed that transfer induced chemotherapy 
resistance and increased survival of the AML cells 61. Another paper also showed transfer of mitochondria from bone marrow stromal cells to leukemic blasts which increased their survival 62. This transfer was dependent on nitric oxide signalling via NADPH-superoxide 2 from the AML cells to the bone marrow stromal cells62.

Conversely, this work also showed that transfer into non-cancerous CD34+ cells did not alter survival of the cells when treated with chemotherapies 62. Most recent evidence indicates that human bone marrow stromal cells have transferred mitochondria to human multiple myeloma cells via tunnelling nanotubules 63. The data suggests that formation of tunnelling nanotubules, and thus transfer of mitochondria, is dependent on expression of CD38 and can be reduced by knocking down CD38 expression 63. These three papers, however, do not provide any information about the likelihood of erythroblasts to be able to transfer mitochondria.

Most of the rationale for exploring mitochondrial transfer in erythroblasts comes from three main areas: cytoplasmic bridge formation, CD38 expression and ability to translate into in vivo human work. For a long time, clinicians have seen cytoplasmic bridges between erythroblasts in routine bone marrow smears82. These are produced between later stages, polychromatophilic and orthochromatophilic (personal communication, Andrew Hall, MIMR, 2016). The relationship between the structure of these inter-erythroid bridges, and tunnelling nanotubules, has not been defined as yet. However, erythroblasts, particularly in the early differentiated states, express CD38 79 and bone marrow based mitochondrial transfer research has indicated a role for CD38 in permitting mitochondrial transfer 63. Finally, bone marrow transplants in people could serve as an in vivo human co-culture, modelling the in vitro and ex vivo experiments for mitochondrial transfer. This ability to translate the research is highly enticing as in vivo human work is lacking in this field. 


\subsection{Aims}

1. Establish ex vivo differentiation of erythroblasts and characterise the cells grown from the differentiation culture method.

2. Identify useful mtDNA polymorphisms and design primers to use in an Allelespecific-blocker assay.

3. Determine a concentration of Deferoxamine to be used in co-culture mitochondrial transfer experiments.

4. Determine if healthy primary erythroblast cells can donate mitochondria to drug treated primary erythroblast cells in co-culture. 


\subsection{Results}

\subsubsection{Mixed-culture cells differentiated from PBMCs morphologically resembled erythroblasts in human bone marrow}

To obtain these erythroblasts in culture, PBMCs were isolated from peripheral blood and cultured for 17 days in complete culture media containing SCF, IL-3, EPO. A regime of Dexamethasone was added every 48 hours for 10 days coupled with estrogen for the final 7 days every 48 hours 87 . The cells that are differentiated from PBMC cultures are mixtures of cell types. Therefore, the cells needed to be characterised for the purposes of this research.

A straight-forward technique to characterise the cells is by morphology. Basic brightfield imaging showed what cells were generated in culture. There were four main cell shapes present: large, non-uniform, mostly adherent cells; medium round suspension cells; smaller round suspension cells; and red blood cells which show a red tint in the images shown (Fig 5.1.A.). This also revealed that some of the cells have cytoplasmic connections between pairs of cells (black arrows, Fig 5.1.A.).

To see if there was any iron present in these cells, slides were sent to Wellington Hospital SCL where they performed a Perl's Stain for iron. An image of bone marrow stained alongside the erythroblast cultures was provided by the hospital as a positive control (Fig 5.1.B). The cells and extracellular matrix stained various red and brown shades whereas the iron clusters stain blue (Fig 5.1.B). Microscopic analysis of cells differentiated from PBMC did not show any classical positive results resembling the control image, however around the dark red nuclei of many cells there appears to be dark blue rims that could be iron (blue arrows, Fig 5.1.B).

Microscopically, erythroblast morphology can be identified using May-GrunwaldGiemsa stain or a commercial alternative called Kwik-Diff. Samples of erythroblast PBMC culture were taken on day 8, 13 and 17, cytospun onto slides and stained with Kwik-Diff (Fig 5.1.C). Three cell populations were identified in the published data of this method, and were also found here: erythroblasts, lymphocytes and macrophages (Fig 5.1.D) 87. Macrophages were large and foamy with a faint pink cytoplasm and a small pink nucleus (yellow arrows). Lymphocytes were small round cells that were 
A
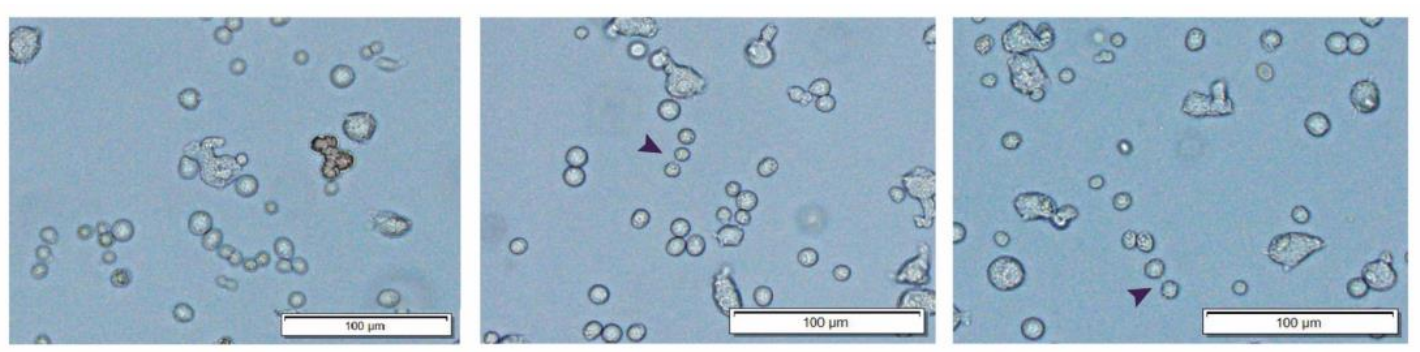

B
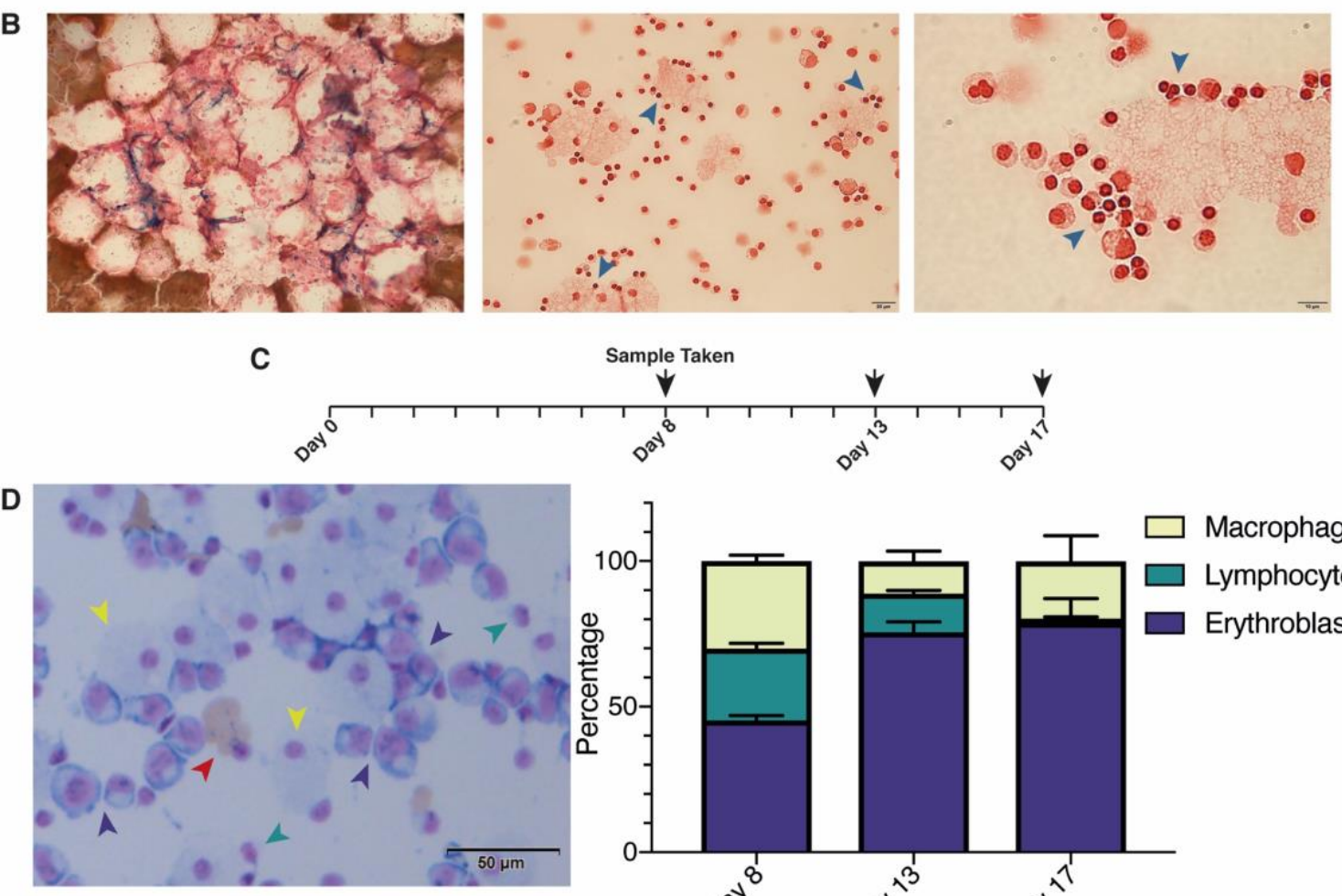

Sample Taken
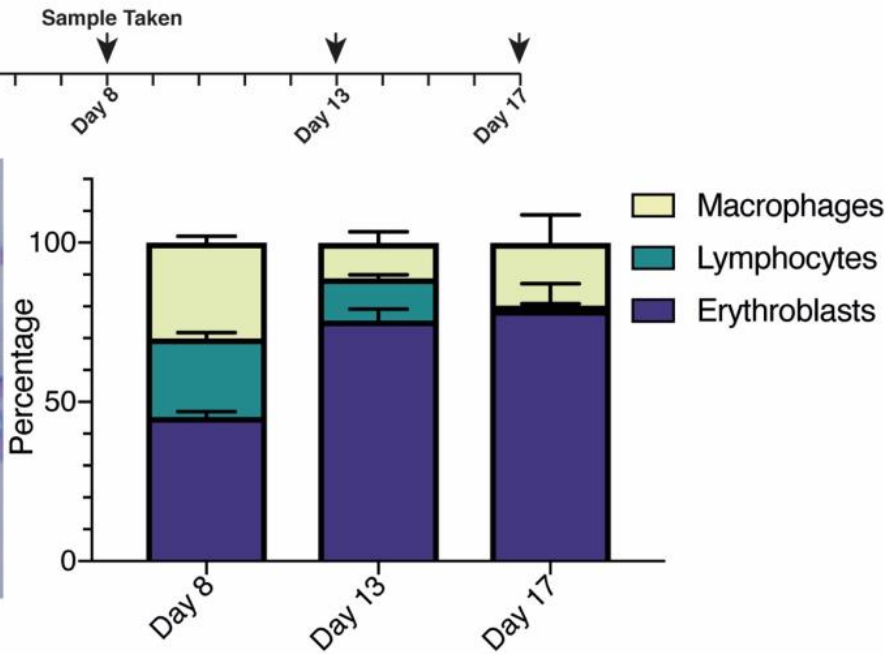

\section{Figure 5.1: Mixed-culture cells from PBMCs morphologically resembled erythroblasts in human bone marrow.}

(A) Three cropped brightfield images taken using 40X magnification. First image on left shows cells healthy in culture. Cells with a red-orange tint are red blood cells. Next two images have black arrows pointing to cytoplasmic connections resembling TNTs. (B) Three images depicting slides stained with Perl's stain for iron clusters. First image on left is a control image taken by Wellington Hospital SCL on an Olympus BX45 20X objective of bone marrow stained alongside the primary cultures. Blue areas indicated presence of iron. Next two images are taken at 40x and 100x magnification respectively of erythroblast cultures. Blue arrows point to examples of blue tinted rims around nuclei or erythroblast cells. $(n=1)(C)$ Schematic showing samples of erythroblast cultures taken over the 17 day period indicated by the black arrows. (D) Image to left shows example image of Kwik-Diff stained cells at Day 8. Yellow arrows indicate examples of foamy macrophages, green arrows show examples of lymphocytes, dark blue arrows show examples of erythroblasts and red arrow shows red blood cells. Graph to right shows enumeration (mean \pm SEM) of cell proportions in the cultures at the time points indicated. Cells were counted using a $4 \times 4$ grid overlaid on the image and counting quadrants for macrophages, erythroblasts and lymphocytes as described further in 2.7.3. $(n=1)$ 
mostly nucleus with minimal cytoplasm (green arrows). Erythroblast in this culture had a variable phenotype as they have several differentiation stages that can be visualised. Pro-erythroblast-like cells have a large nucleus surrounded by white Golgi and a deep blue outer rim detailing the edge of the cytoplasm (blue arrows) 122. Throughout the differentiation process in culture, erythroblasts began to shrink in size, reduced the size of the nuclei and Golgi, and were mostly identified by the remaining presence of the blue rimmed cytoplasm (Fig 5.1.D) 122. Proportions of each cell population were counted, and data collected as shown (Fig 5.1.D). At Day 8 in the culture, close to $50 \%$ of cells present are erythroblast-like in morphology (mostly at the pro-erythroblast stage) and this proportion continues to grow, to hit a maximal $80 \%$ frequency at 17 days (Fig 5.1.D). Interestingly, lymphocytes and macrophages take up the other $50 \%$ of the culture early on and while macrophages persist longer into the culture, lymphocytes appear to disappear completely by the end point of the culture method (Fig 5.1.D). Although only analysed using these techniques once, the data replicate what was shown in the original methodology, and provide strong evidence that the majority of cells in this mixed culture were erythroblast-like87.

\subsubsection{Mixed-culture cells expressed common erythroblast surface markers detected by flow cytometry}

While microscopy can be used as one approach to characterise cell type, flow cytometry is another useful approach. Previous data published has created an in-depth profile of human erythroblast cell surface expression at different stages of erythropoiesis 79. A carefully selected panel of four antibodies, based on this published data, was used to confirm the differentiation state of the cell populations during culture. The markers selected were CD117 (stem cell factor receptor), CD38 (cyclic ADP ribose hydrolase), CD71 (transferrin receptor) and CD235a (glycophorin A) 79. CD34+ progenitors and pro-erythroblasts can be identified by expression of CD117, CD38 and CD71, and coexpression of CD71 and CD235a indicate presence of later stage erythroblasts such as basophilic, polychromatophilic and orthochromatophilic79.

Based on size, three populations of cells were identified, originally labelled populations 1, 2 and 3, were analysed for erythroid marker expression (Fig 5.2.A) at three timepoints in the erythroblast culture: Days 3,10 and 15. Population 1 was the only population present over the three time-points and begun at Day 3 with a quarter of cells 
A

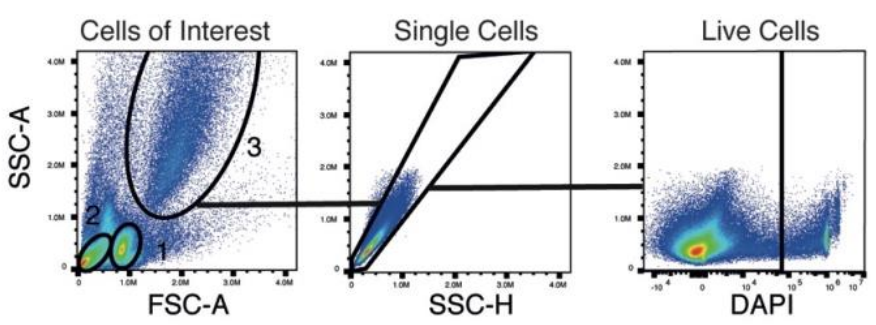

B
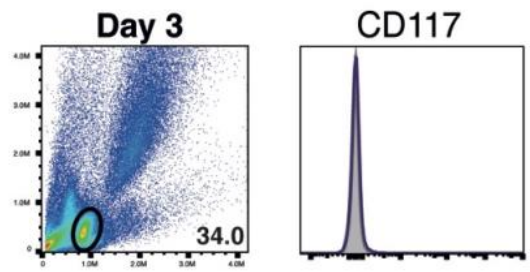

CD38
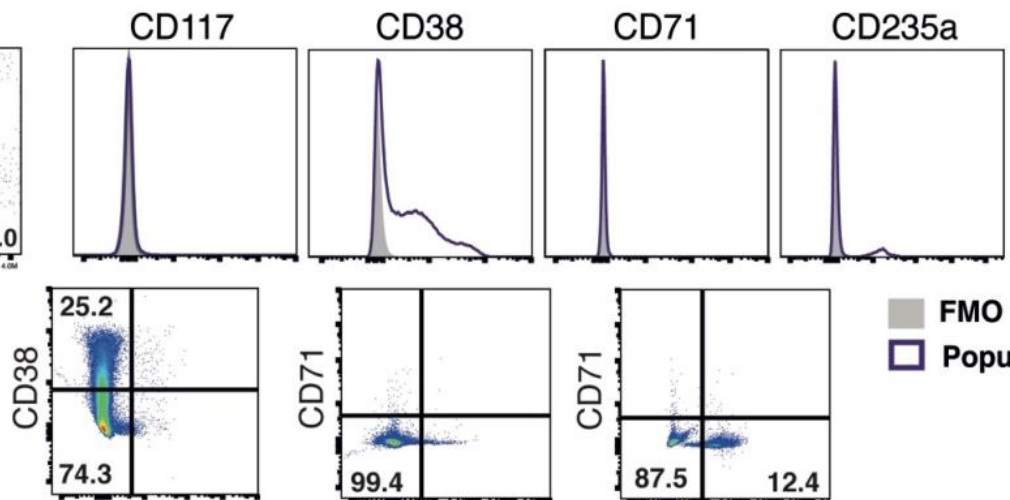

Day 10
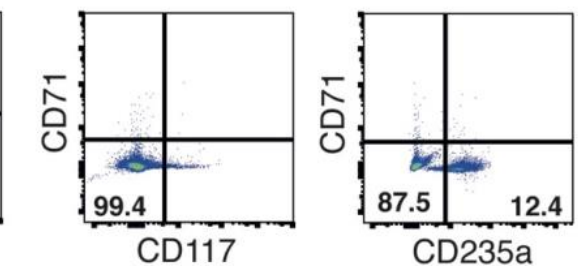

FMO

$\square$ Population 1
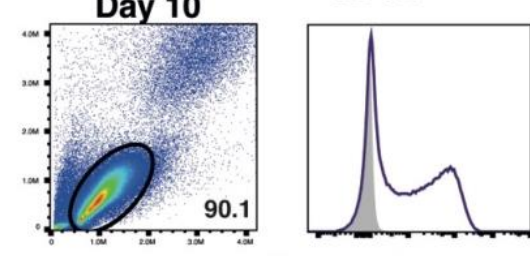

CD117

CD235a
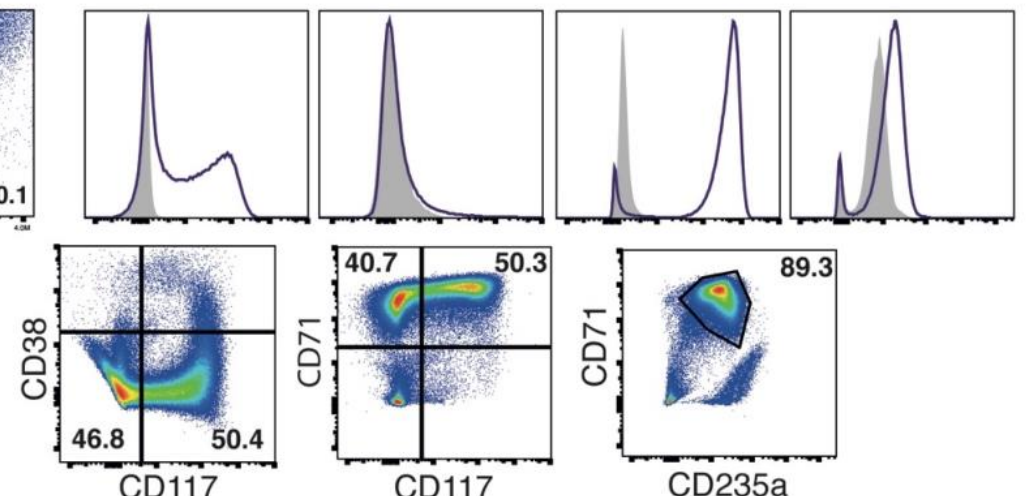

Day 15
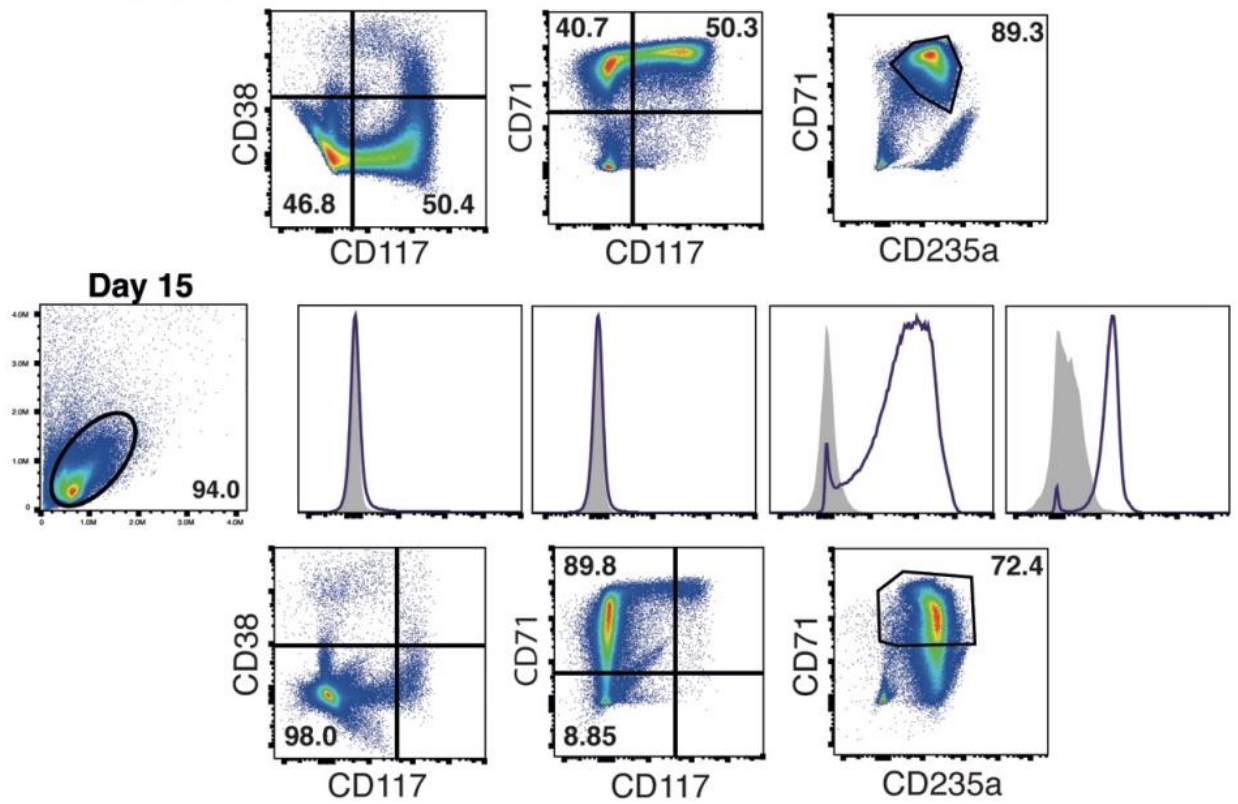

Figure 5.2: Mixed culture cells expressed common erythroblast surface markers detected by flow cytometry.

(A) Example gating strategy used to present histograms shown in following sub-figures. In subfigures at specific times the cells of interest gate were altered accordingly to fit the populations morphology. Cells were first gated on cells of interest, then single cells gated out and finally on live cells by using DAPI as the viability stain. (B-D) Histogram analysis of four surface markers - CD117, CD38, CD71 and CD235a for 3 populations present over 15 days of culture (populations 2 and 3 were not present at day 15). FMO (fluorescence minus one, or total fluorescence in sample except the fluorophore in question) is in grey and population is coloured as per legend below histograms. 
C
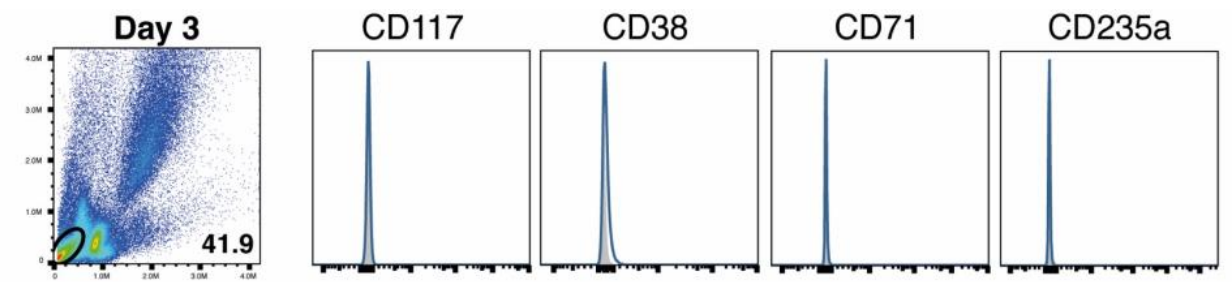

Day 10
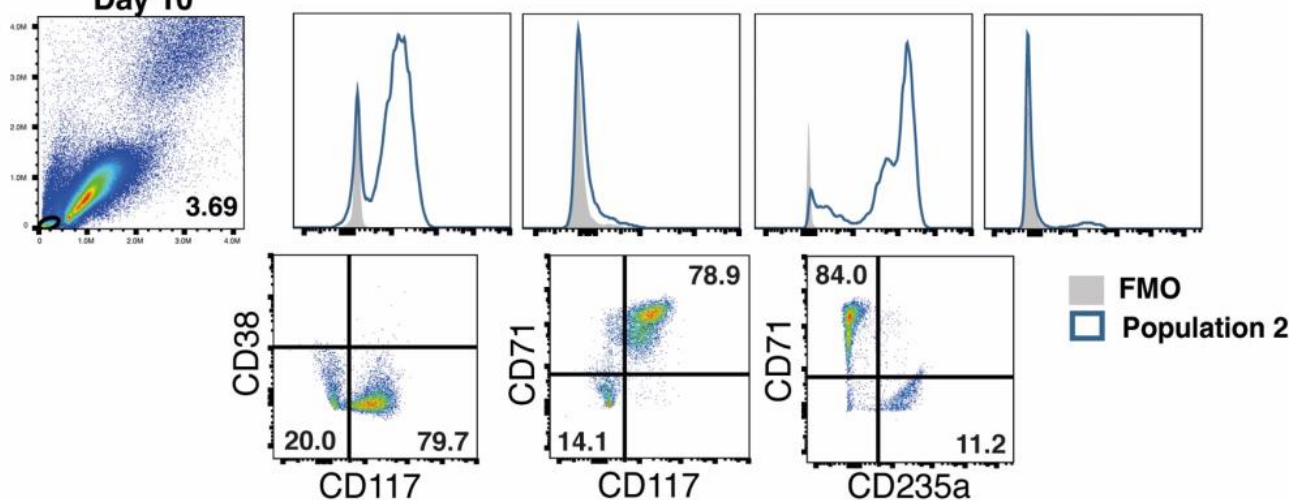

D
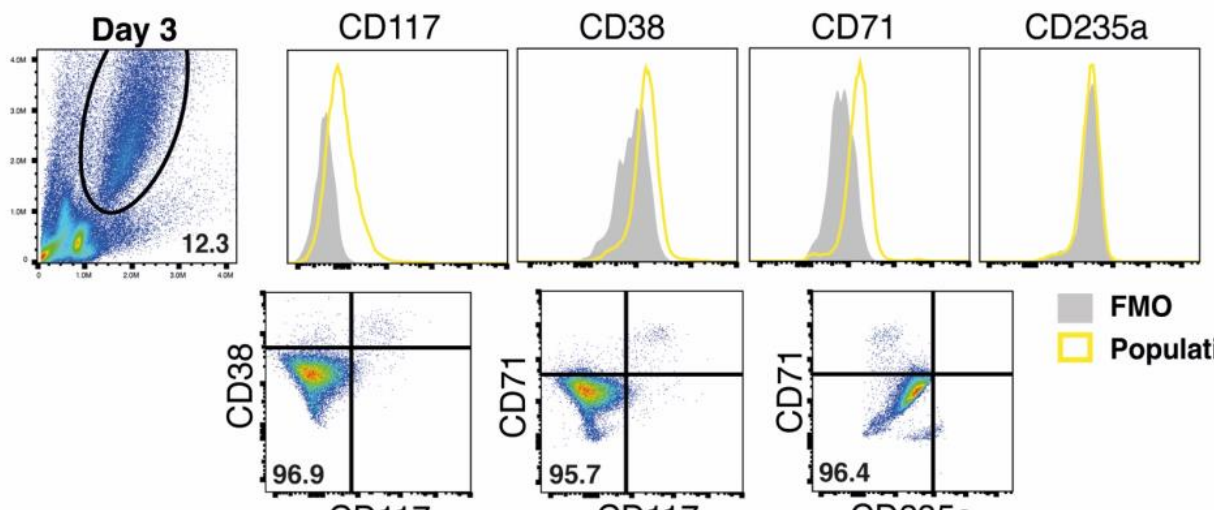

FMO

Population 3

96.4

$\overline{\mathrm{CD}} 235 \mathrm{a}$
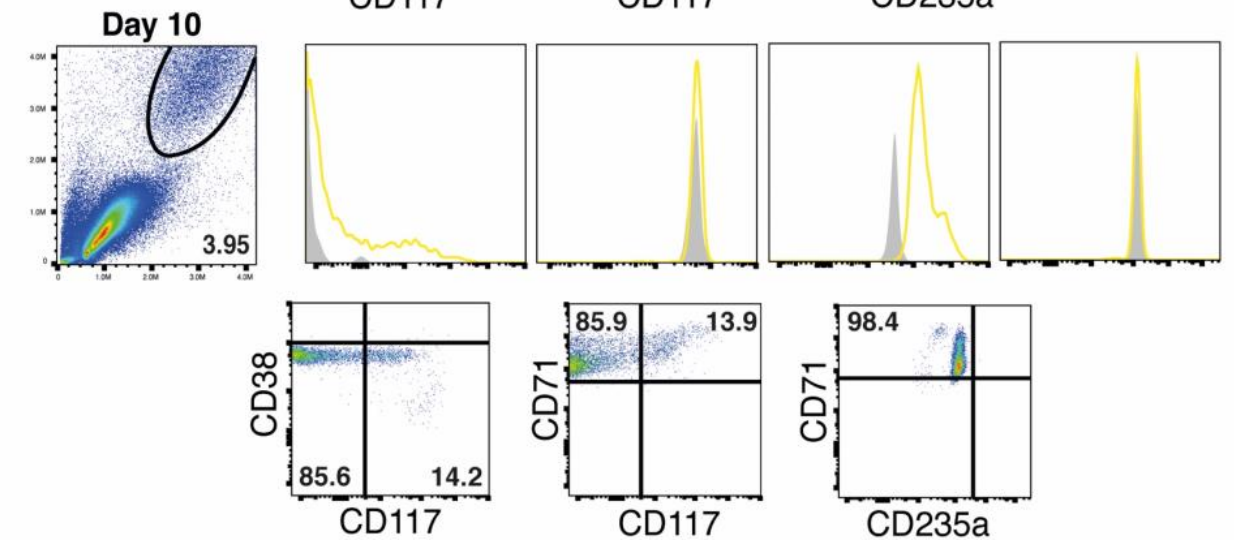

(B-D) continued... Three graphs under each applicable day show double staining for markers where co-expression relates to a cell type. Co-expression of CD38 and CD117 indicates CD34+ progenitors or pro-erythroblasts. CD71 and CD117 co-expression also indicate pro-erythroblasts. CD71 and CD235a co-expression indicate presence of basophilic, polychromatophilic to orthochromatic stages of erythroblasts. CD71 is also expressed on macrophage-like cells. $(\mathrm{n}=1)$ 
in this group expressing CD38 (25.2\%) and a small number of cells (12.4\%) expressing CD235a (Fig 5.2.B). These CD38+ cells could possibly be a small number of activated lymphocytes, or CD34+ erythroid progenitors. The CD235a+ cells could be large clumps of red blood cells that remained from the PBMC isolation 79,123. By Day 10, population 1 had grown considerably in FSC and SSC, 90\% of the cells expressed CD71 and $50 \%$ of these cells also expressed CD117. All the cells that were CD71+ were also positive for CD235a to low levels (Fig 5.2.B). This expression profile was indicative of almost all stages of erythroblasts being present, from pro-erythroblasts to poly-and orthochromatophilic cells 79. At Day 15, cells in population 1 have lost CD117 expression, and are $80 \%$ double positive for CD71 and CD235a, indicating further differentiation through erythropoiesis (Fig 5.2.B). It is very convincing that population 1 cells are the erythroblast-like population in these cultures in size and expression profiles.

Population 2 was the smallest of the three populations in size and reduced in number markedly from Day 3 (41.9\%) to Day 10 (3.69\%) (Fig 5.2.C). At day 3, cells in population 2 did not show any positive expression for the four markers chosen, however at Day $10,80 \%$ of the small proportion of cells were double positive for CD71 and CD117 (Fig 5.2.C). Eleven percent of the cells were also positive for CD235a. Their size, drastic reduction in numbers and expression profile indicate that these cells were most likely debris from dying cells and possibly residual red blood cells that were maintained throughout the culture.

The third population defined in these cultures were much larger in size and highly autofluorescent. Population 3 did not express detectable levels of any the four markers at day 3 compared to their FMO (fluorescence minus one) controls. At day 10 almost $100 \%$ of these large cells expressed CD71, of which 14\% were also expressing CD117 (Fig 5.2.D). CD71 is commonly expressed on macrophages, not just erythroid cells and therefore is a large indicator that these large cells are macrophage-like, consistent with the morphological analysis 124 . The small proportion of these cells which are also CD117 positive could be more monocyte-like 125.

It is important to note that expression levels will not be identical culture to culture due to the single replicate of this data, however the trends will stay the same. These flow cytometry data corroborated with the Kwik-Diff microscopy analysis that macrophage- 
like cells are present and that erythroblast-like cells are not only present but are differentiating through erythropoiesis in these cultures.

\subsubsection{A mitochondrial DNA polymorphism suitable for detecting transfer in the primary cell model was identified}

Detection of mitochondrial transfer between erythroblast cells in these cultures relied heavily on the detection of useful SNPs in the mitochondrial DNA between donor and recipient populations. To identify polymorphisms in potential donors of PBMC, Sanger sequencing was conducted on whole blood PCR mitochondrial genome products of 10 blood donors. In accordance with the ethics approval associated with their participation in this study, each donor was given a specific identifier, MPB-01 through MPB-10 and the hypervariable regions of the mitochondrial genome in each donor were sequenced 126.

One useful polymorphism was identified that would fit into the previous ASBqPCR designs with minor modifications, m.152C $>$ T (Fig 5.3.A). A variation of the allelespecific-blocker qPCR assay was designed to detect this SNP in MPB-09 mtDNA (Fig 5.3.B). Finally, within the probe there is another polymorphism m.217C $>\mathrm{T}$ which should aid the specificity of the probe (Fig 5.3.B).

The basic pipeline of the primary erythroblast co-culture to detect mitochondrial transfer was as follows: a male cell (MPB-09) with the mitochondrial polymorphism m. $152 \mathrm{C}>\mathrm{T}$ and stained with proliferation dye, was co-cultured with a female cell (MBP10) that did not have the polymorphism, stained with another proliferation dye. Female cells were also treated with a drug, Deferoxamine. After 48 hours, both populations were separated using fluorescence associated cell sorting cytometry, and DNA extracted. ASBqPCR was then used to detect mitochondrial transfer in the female MPB10 population and control male MPB-09 population (Fig 5.3.C). 
A

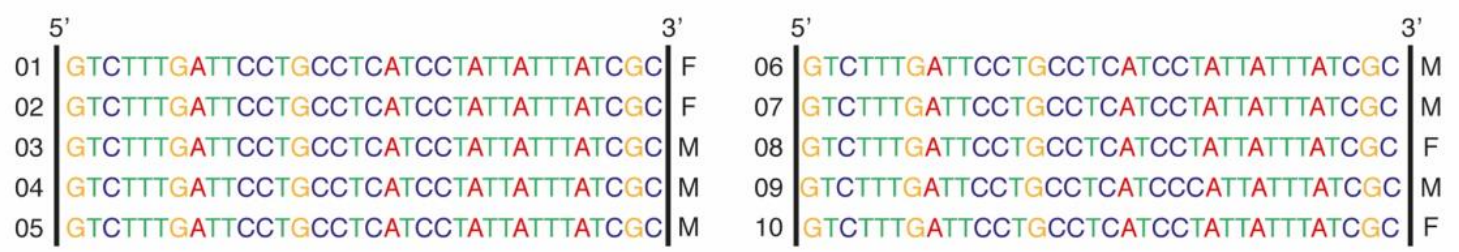

B
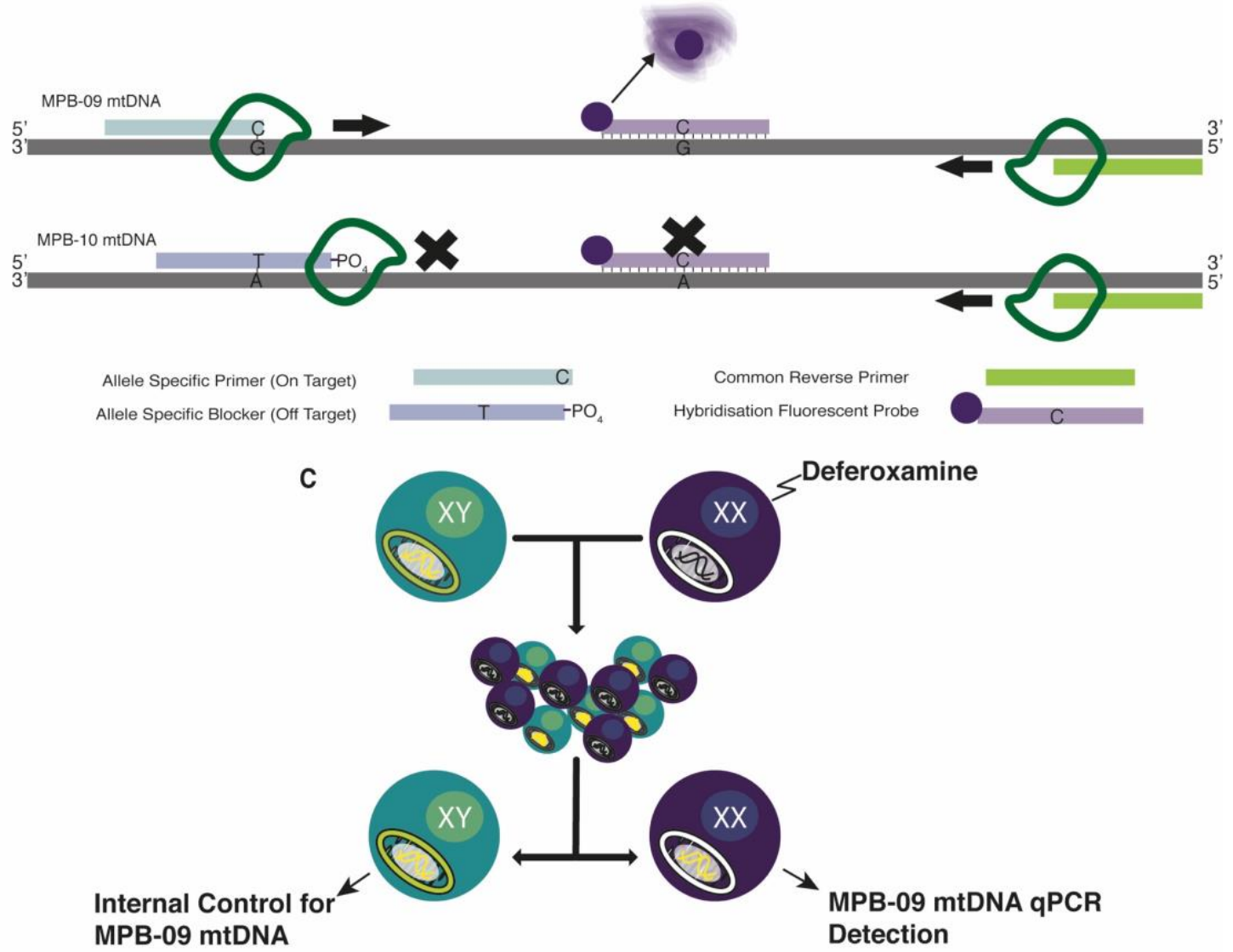

Figure 5.3: A mitochondrial DNA polymorphism suitable for detecting transfer in the primary cell model was identified.

(A) A screen of 10 volunteers was performed. Two $\mu \mathrm{L}$ of blood obtained by fingerstick, amplified using PCR and Sanger sequenced. One mtDNA polymorphism was found in male MPB-09 at position $152 \mathrm{~T}$ to C. (B) Schematic depicting how the ASBqPCR detected MPB$09 \mathrm{mtDNA}$ in an MPB-10 background. The background polymorphism is bound by a blocker primer with a 3' phosphate group preventing extension by DNA polymerase, and preventing release of fluorescence from the probe downstream. The fluorescent probe has a nucleotide mismatch that should reduce binding to off target mtDNA. Both on- and offtarget templates are amplified using the common reverse primer. The target sequence is bound by a specific primer which allows DNA polymerase extension and release of fluorescence read by the qPCR machine. Adapted from Morian et al., 2009. (C) Schematic detailing the parameters required for co-culturing primary erythroblasts to detect mitochondrial transfer. A male erythroblast population with a detectable polymorphism in the mtDNA (and stained in a cell trace dye) is co-cultured with a female erythroblast population that does not have the mtDNA polymorphism, stained with a different cell trace dye. After 48 hours in co-culture the two cell populations are separated out using a flow cytometric cell sorter. DNA is extracted and analysed using ASBqPCR to determine whether the male mtDNA polymorphism can be detected in the female erythroblasts. 

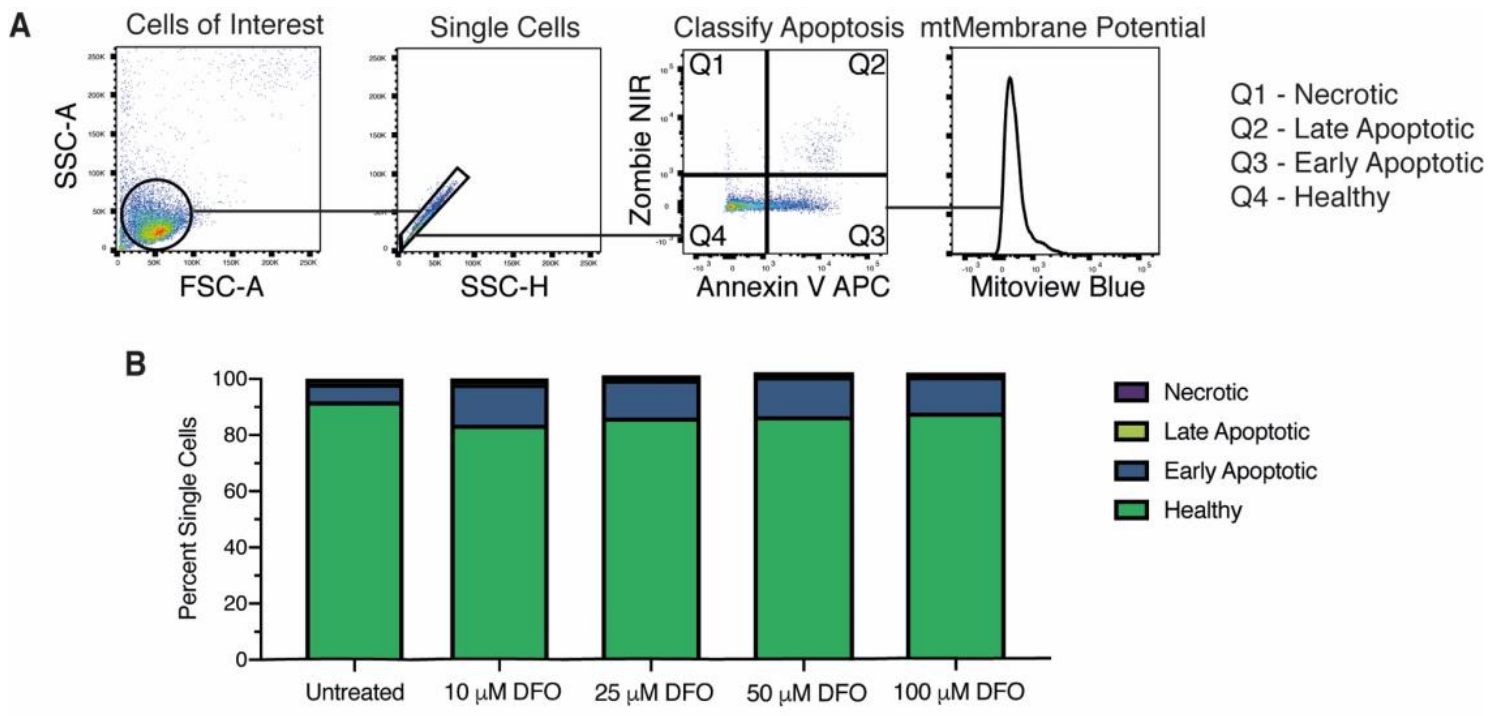

Figure 5.4: Deferoxamine increased erythroblast culture apoptosis after a 24-hour treatment.

(A) Gating strategy used to classify apoptotic stage - annexin negative cell death, late apoptotic, early apoptotic. (B) Graphs of untreated cells and 4 concentrations of Deferoxamine after 24 hours showing the percentages of each apoptotic populations from flow plots. Annexin negative cell death not included in graphs because not visible due to such small percentages i.e $>0.3 \%$. $(\mathrm{n}=1)$ 


\subsubsection{Deferoxamine increased erythroblast culture apoptosis after 24-hour treatment}

To elucidate a drug concentration that would induce stress to a level that might lead to mitochondrial transfer, a dose titration, using 4 concentrations between $10 \mu \mathrm{M}$ and 100 $\mu \mathrm{M}$, was conducted on the erythroblast ex vivo cultures. Due to ethical concerns about the volume of blood to be donated, as well as time constraints this was not completed as a time-course but only assessed the apoptotic phenotype of cells 24 hours after treatment. This time point was equivalent to when drug treated cells would go into coculture with the potential donor erythroblast cell population.

The apoptosis gating strategy included analysis of 4 states, annexin negative cell death, late apoptosis, early apoptosis and healthy cells (Fig 5.4.A). Proportions of cell in each apoptotic state were calculated for each concentration of DFO, and an untreated control population, and results shown in graphical form (Fig 5.4.B). In the untreated control, around $90 \%$ of cells remained healthy in culture. With each concentration of DFO treatment, the proportion of healthy cells decreased (Fig 5.4.B). This drop in healthy cells corresponded to an approximate $18-20 \%$ increase in early apoptosis for all DFO concentrations. Late apoptotic and cells that underwent annexin negative cell death were at very low proportions in all samples analysed at this time point (Fig 5.4.B).

Due to the inability to foresee the future effects each drug concentration may have on these cells and the similarity in effect at this point for each option, $25 \mu \mathrm{M}$ DFO was chosen for future use in order to match the highest concentration used in the HEL-HL60 cell line co-culture system.

\subsubsection{Erythroblast cultures did not completely recover 48-hours after 24-hour deferoxamine treatment}

To understand the recovery ability of the erythroblast cultures after $25 \mu \mathrm{M}$ DFO treatment, a recovery model was assessed. This entailed treating the MPB-10 cells with $25 \mu \mathrm{M}$ DFO, or leaving them untreated, for 24 hours then replacing the drug-treated media with fresh media. Cells were left for 48 hours before assessing apoptosis and mitochondrial membrane potential by flow cytometry (Fig 5.5.A). Both the erythroblast and macrophage populations were analysed independently for extent and stage of apoptosis (Fig 5.5.B). 
Again, cells that died by annexin negative processes were not visible in graphs due to their small proportions. Interestingly, there was no difference between the erythroblast population overall viability of untreated and $25 \mu \mathrm{M}$ DFO conditions, suggesting they were not affected by, or more likely easily recovered from, DFO treatment, in the 48hour period (Fig 5.5.C). Comparatively, the macrophage population decreased from approximately $85 \%$ healthy cells untreated to $75 \%$ healthy cells when treated with 25 $\mu \mathrm{M}$ DFO. The proportion of early apoptotic cells increased by about $5 \%$ and proportion of late apoptotic cells reached approximately $3 \%$ in the $25 \mu \mathrm{M}$ DFO treated condition (Fig 5.5.C). This indicates that macrophages were more affected by this treatment in the long term compared to the erythroid cells in the mixed cultures.

To further investigate the individual apoptotic populations, the Mitoview blue stain was included to look at mitochondrial membrane potential. If mitochondria maintain their membrane potential the mitochondria stain blue and when they lose membrane potential, the stain disappears. Flow cytometry analysis shows that the healthy and annexin negative cell death populations, regardless of treatment, share similar mitochondrial potential phenotypes (Fig 5.5.D). Notably, more cells in the early apoptotic $25 \mu \mathrm{M}$ DFO treated erythroblast and macrophage populations appeared to lose their mitochondrial membrane potential, indicated by a smaller right peak and larger left peak (Fig 5.5.D). Additionally, membrane potential reduced in cells undergoing late apoptosis after $25 \mu \mathrm{M}$ DFO compared to untreated controls (Fig 5.5.D). These results indicated that even though the $25 \mu \mathrm{M}$ DFO treatment may not have affected the apoptotic proportions drastically, particularly for the erythroblast population, there was still an effect on the mitochondrial membrane potential for these cells, which they did not recover from on their own. This may provide the opportunity for transfer of mitochondria to assist with recovery. 
A

$\begin{array}{cccc}\text { T-24 } & \text { T0 } & \text { T48 } \\ \text { Dose } & \text { Replace } & \text { Flow } \\ \text { with DFO } & \text { Media } & \text { Cytometry } \\ \text { Assessment }\end{array}$

B

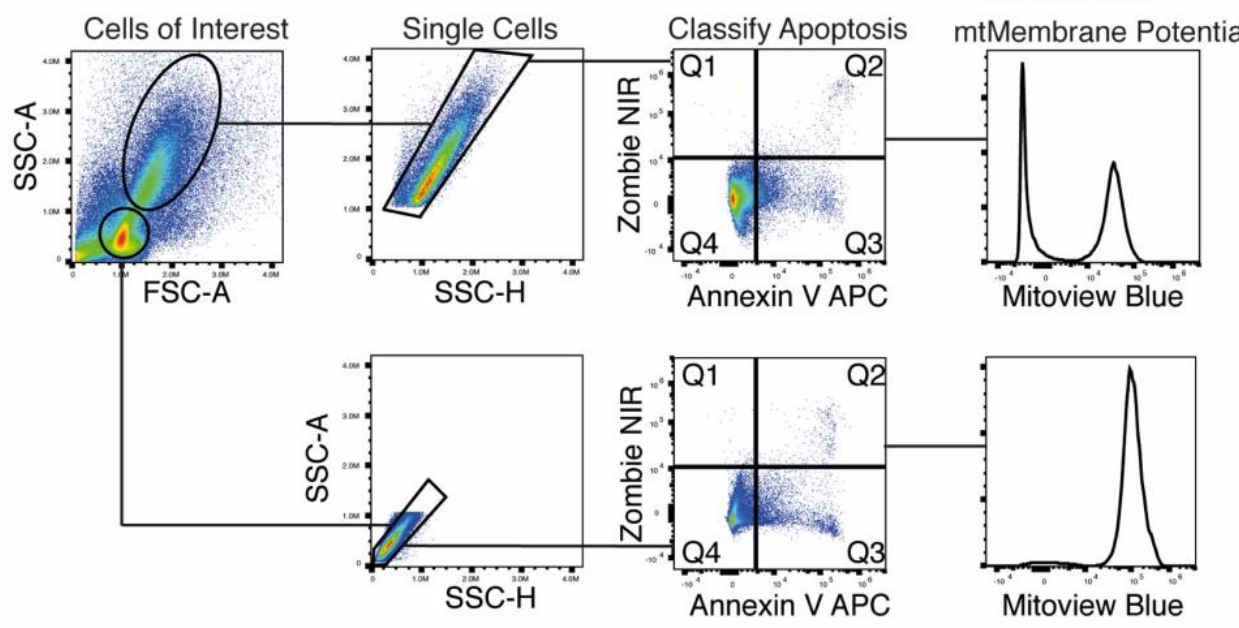

C

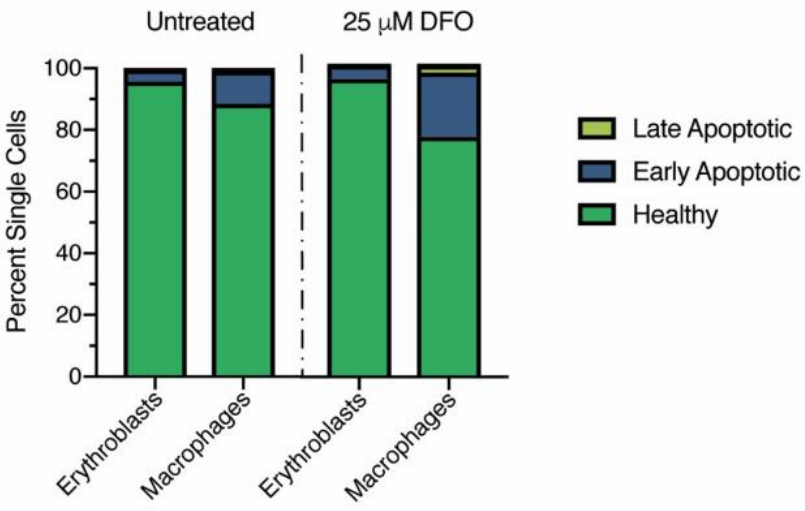

D

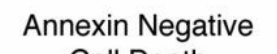

Untreated

Erythroblasts

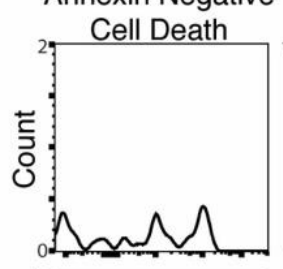

Late Apoptotic Early Apoptotic

Healthy Erythroblasts
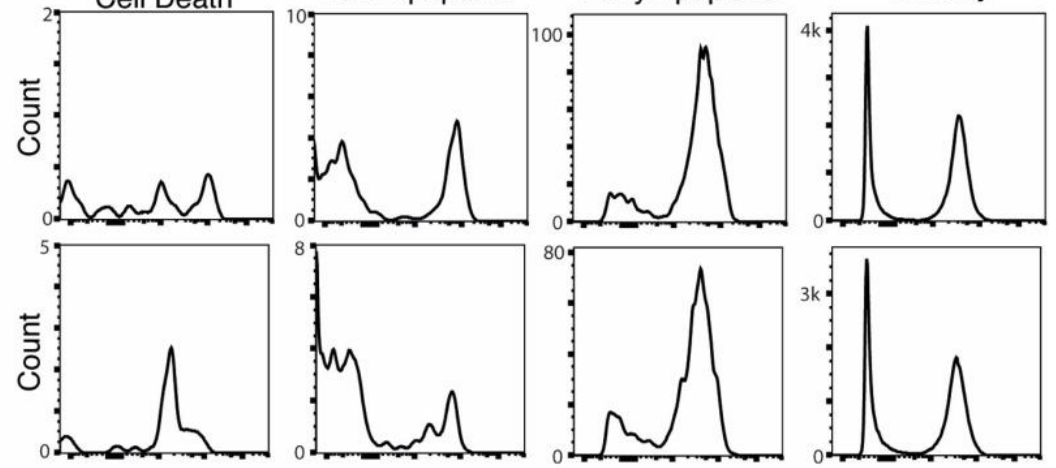

$25 \mu \mathrm{M}$ DFO
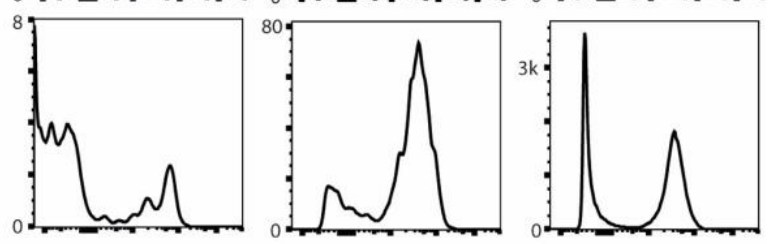

Untreated Macrophages
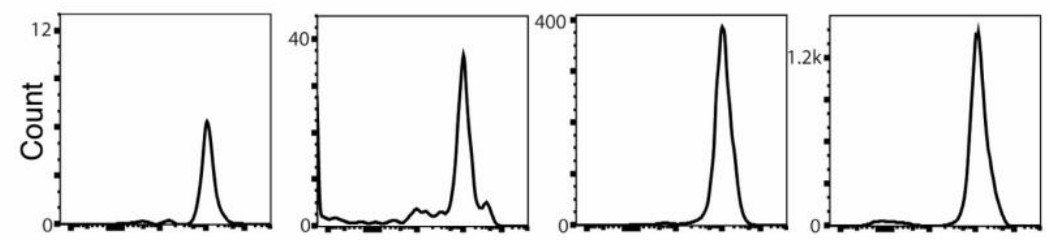

$25 \mu \mathrm{M}$ DFO Macrophages
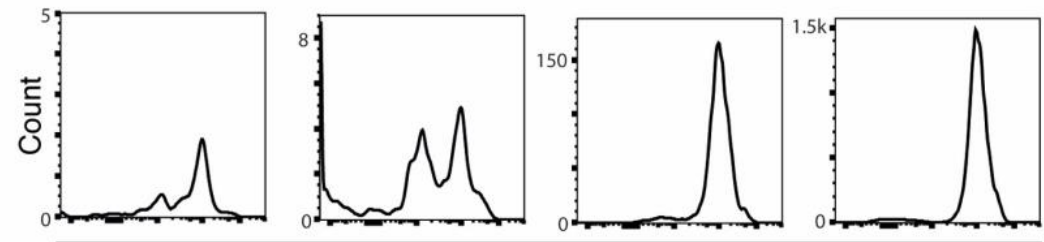

Mitoview Blue 
Figure 5.5: Erythroblast cultures did not fully recover 48 hours after a 24-hour Deferoxamine treatment.

(A) Schematic depicting the experimental flow for the recovery from injury experiment. Cells were dosed with $25 \mu \mathrm{M}$ of DFO 24-hours before replacement in fresh media only and after 48 hours apoptosis and mitochondrial potential were assessed using flow cytometry. (B) Gating strategy used to assess apoptosis and mitochondrial potential of erythroblast and macrophage populations present in the culture. Populations labelled as per Figure 5.4.A (C) Graphs depicting percentages of apoptotic populations of both erythroblasts and macrophages present in treated and untreated co-cultures. (D) Histograms depicting Mitoview blue fluorescence for cells in each apoptotic population for untreated and $25 \mu \mathrm{M}$ DFO treated erythroblasts and macrophages. Y scales are shown using 0 and the largest number for each graph for comparison. $(\mathrm{n}=1)$ 


\subsubsection{Co-culturing Deferoxamine-treated erythroblasts with untreated erythroblasts did not increase viability above controls}

Co-culture was able to increase viability of HL-60 مo cells when deprived of pyruvate and uridine, and increase viability of HEL cells treated with DFO in vitro. Therefore, I wanted to assess if the same could be seen for DFO treated erythroblast cells in coculture. The gating strategy used included an antibody for CD235a, this marker is specific to erythroid cells and therefore allowed visualisation of where erythroblasts sit in the FSC/SSC plots, to ensure analysis of the correct cell type (Fig 5.6.A). Gating also included the use of both strict DAPI negative and DAPI negative and intermediate gates (Fig 5.5.A).

Regardless of the viability gate (strict or generous), there was no significant difference in the viability between MPB-09 cells co-cultured with $25 \mu \mathrm{M}$ DFO treated, or with untreated MPB-10 cells. Both were maintained above 95\% (Fig 5.6.B). Likewise, there was no difference between the viability of the MPB-10 $25 \mu \mathrm{M}$ DFO treated or untreated cells 48 hours after co-culture and no difference compared to the recovery model control of Zombie negative cells, also above 95\% (Fig 5.6.C). This is not unexpected as the recovery model showed that the cells were able to recover their viability on their own, independent of co-culture (Fig 5.5.C).

\subsubsection{Deferoxamine-treated erythroblasts did not receive detectable mitochondrial DNA from untreated erythroblasts in co-culture}

The response of the ASBqPCR to primary human DNA and mtDNA must be understood before transfer of mitochondrial DNA can be detected. To do this I used an artificial spike-in, where titrated amounts of target DNA were added to controlled amounts of background, off-target DNA. Alongside these samples a control 100\% background unmanipulated sample was tested to show the background of MPB-10 amplification. 

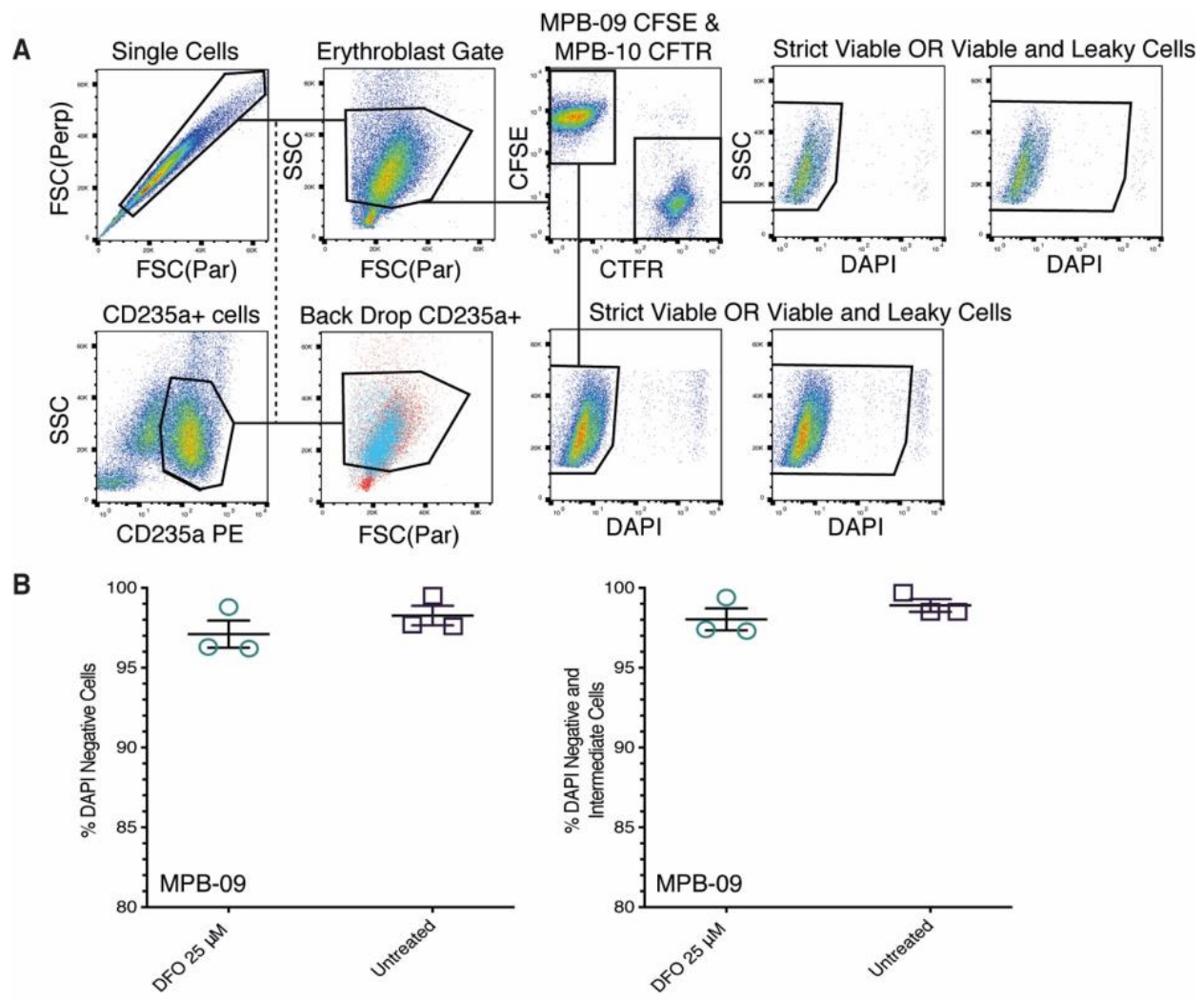

C
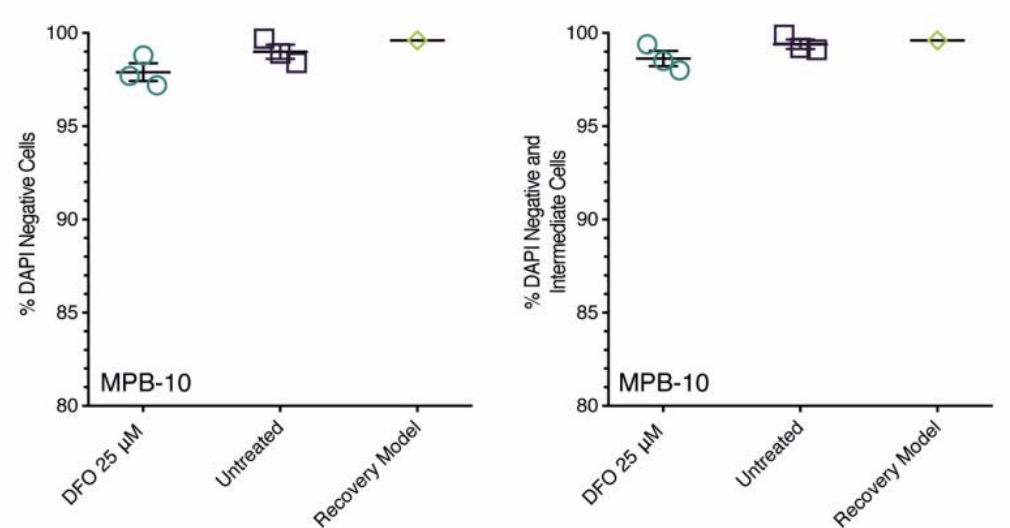

Figure 5.6: Co-culture after Deferoxamine treatment did not increase viability above controls.

(A) Gating strategy for assessing viability of MPB-09 and MPB-10 cells 48 hours after coculture during the sorting process. From single cells, CD235a staining was used to identify the location of erythroblasts in the FSC/SSC plots but not directly included in the analysis strategy (dotted line). Both a strict DAPI negative and DAPI negative and intermediate cells gate was included in analysis. (B) Left graph shows strict DAPI negative viability of 3 replicates for MPB-09 cells that were co-cultured with either Untreated MPB-10 cells or $25 \mu \mathrm{M}$ DFO treated MPB-10 cells. Right graph shows the same conditions but using the DAPI negative and intermediate gating strategy. (C) Left graph shows strict DAPI negative viability of MPB-10 cells that were either treated with $25 \mu \mathrm{M}$ DFO or untreated $(n=3)$ alongside the erythroblast population, from the $25 \mu \mathrm{M}$ DFO recovery model, that were Zombie negative as a comparison of how the cells could recover on their own $(n=1)$. Right graph shows the same conditions but with DAPI negative and intermediate gating for the sorted populations. Data shown as mean \pm SEM. ( $\mathrm{n}=3$ independent experiments) 
Results from this test show that detection of mtDNA was more limited than when using cell line DNA, with the sensitivity of detection around 0.1\% target MPB-09 mtDNA ompared to previous $0.001 \%$ target HL-60 mtDNA (Fig 5.7.A). This is most likely due to heteroplasmy of human mitochondrial genomes increasing the background signal 127.

The standard curve, ran alongside the co-cultured and separated erythroblast DNA, and the calculation of the log-linear regression line were therefore only fitted to the first 3 titrations $(10 \%-0.1 \%)$, to obtain the most accurate representation of target mtDNA present within the limits of the assay (Fig 5.7.B).

The Ct amplification of MPB-10 DFO treated and untreated DNA samples did not appear earlier than the limit of the 100\% MPB-10 background detection. Nested t-test statistical analysis of the replicates did not show any significant difference between the overall conditions (Fig 5.7.C). This indicates that no mitochondrial DNA has been transferred to a detectable level from MPB-09 cells into MPB-10 DFO treated cells, compared to untreated MPB-10 cells. There is also significant variation between replicates of both treated and untreated MPB-10 DNA samples $(\mathrm{p}<0.05)$, possibly due to the nature of primary cells (Fig 5.7.C).

The calculated mean percent target DNA also did not indicate any MPB-09 mtDNA above the $0.1 \%$ limit of detection. All samples of MPB-10 DNA contained between $0.01-0.055 \%$ MPB-09 mtDNA which may be heteroplasmic genomes present within the intrinsic MPB-10 cells (Fig 5.7.C). Interestingly, the control MPB-09 samples of DNA that were co-cultured with DFO treated MPB-10 cells displayed more variation within replicate amplification when compared to the MPB-09 cells co-cultured with untreated MPB-10 cells (Fig 5.7.C).

MPB-10 erythroblast cells were treated with $25 \mu \mathrm{M}$ DFO for 24 hours before adding to co-culture with untreated MPB-09 erythroblast cells. Prior to addition to co-culture, MPB-10 and MPB-09 cells were stained with different proliferation dyes. Co-cultures were left for a further 48 hours at which point MPB-10 and MPB-09 were separated using fluorescence associated cell sorting. DNA was extracted from separated populations for ASBqPCR detection of mtDNA transfer. The gating strategy used to isolate the erythroblasts from these co-cultures also included the CD235a antibody. However, this was used purely to confirm the location of the gate and not in the final 
sort strategy (Fig 5.7.D). Each population was first sorted through an enrich phase (yielding around $2 \%$ contamination) then sorted a second time through a 1-drop pure phase to obtain the highest levels of purity. Purity analysis of 1000 cell samples of the separated populations show a very high level $>98.5 \%$, except for replicate 3 of $25 \mu \mathrm{M}$ DFO treated co-culture which had purity of $95.9 \%$ for the MPB-09 population and 96.4\% for the MPB-09 population (Fig 5.7.E). This did not appear to have affected the MPB-10 population in terms of mtDNA amplification outside the other replicates, as demonstrated in cell line analysis. It is unclear if this impure sort is implicated in the variable amplification seen in the control MPB-09 population. Further investigation is needed to explore how contamination effects the positive control or if the variation seen is due to a biological effect in the co-culture system when DFO treated cells are present. 
A

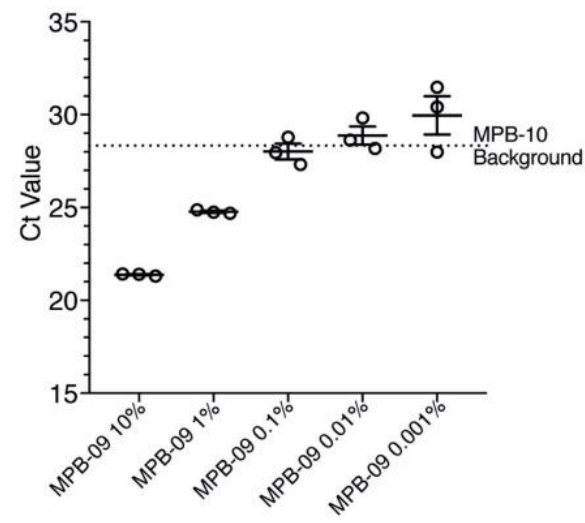

B

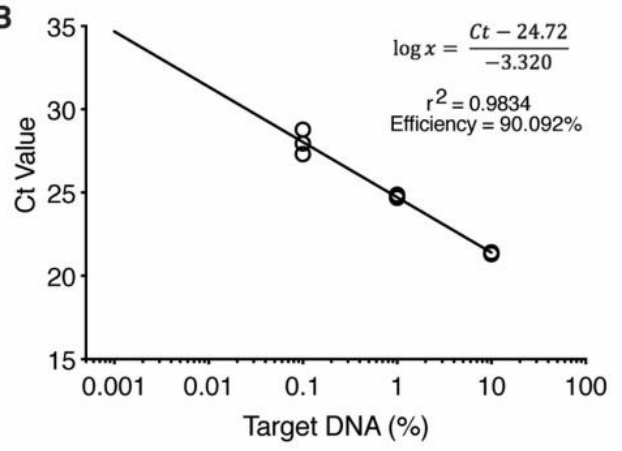

C
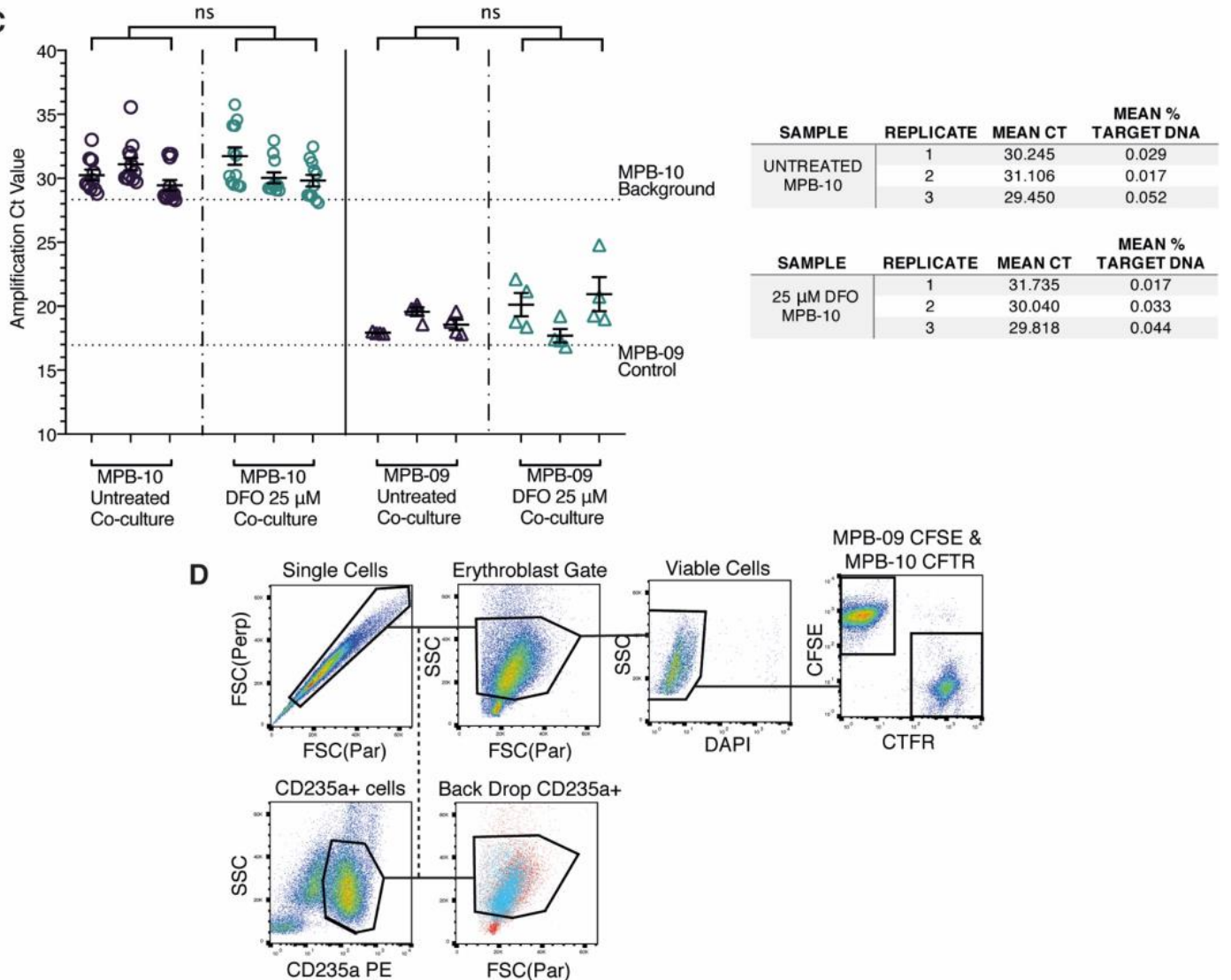

E

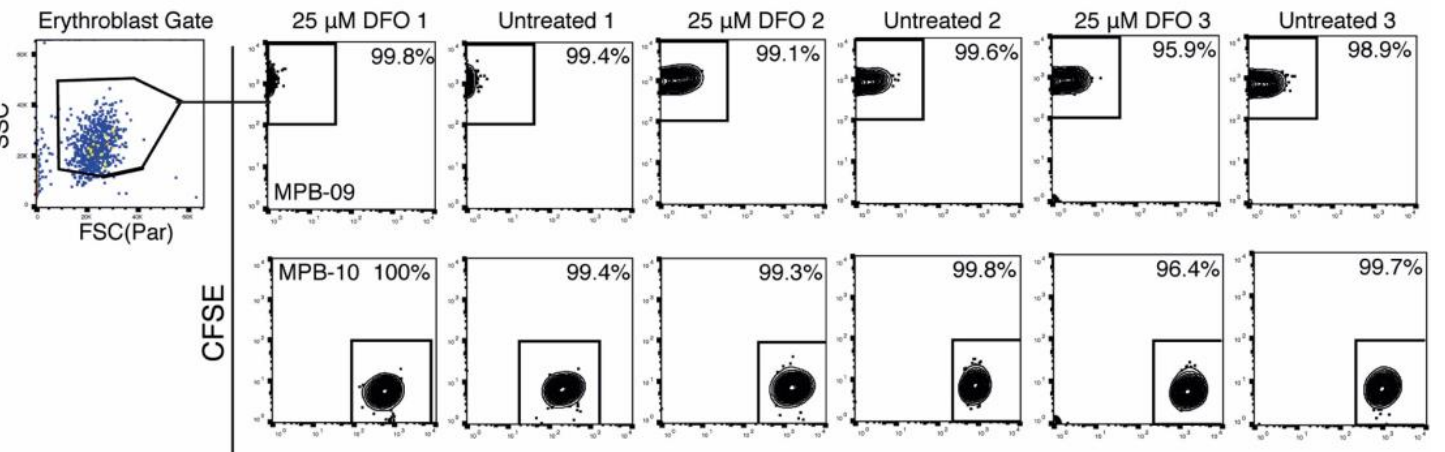

Cell Trace Far Red 


\section{Figure 5.7: Deferoxamine-treated erythroblasts did not receive detectable mitochondrial DNA from untreated erythroblasts in co-culture.}

(A) Standard curve raw Ct points from an artificial spike-in of 10\%-0.001\% MPB-09 DNA into an MPB-10 background. Horizontal dotted line showed the limit of detection when $100 \%$ MPB10 background DNA was amplified on its own. (B) Standard curve correlating percent target DNA present, to amplification Ct, using the artificial spike-in Ct values (only including the first three points where detection was valid). A log-linear line was fit to the data to create an equation to calculate percent target DNA. The $r 2$ value for this curve and the efficiency of the qPCR reaction are provided. (C) Both MPB-10 and MPB-09 samples for untreated and $25 \mu \mathrm{M}$ DFO treated (3 biological replicates each) were run through the ASBqPCR to detect MPB-09 target mtDNA and their amplification $\mathrm{Ct}$ values are shown in the graph to the left. For each replicate 12 replicates wells of DNA were analysed for each MPB-10 sample to detect mtDNA transfer and 4 replicate wells of DNA were tested for MPB-09 as a control. Two horizontal lines show the limit of detection for a 100\% MPB-10 background and the point at which $100 \%$ MPB-09 (positive control) amplified for reference. A nested t-test assessing the difference between the untreated and $25 \mu \mathrm{M}$ DFO treated MPB-10 replicate samples showed no significance. Data shows mean \pm SEM. To the left, tables show the mean $\mathrm{Ct}$ amplification and mean calculated percent target MPB-09 mtDNA in MPB-10 samples that were untreated or 25 $\mu \mathrm{M}$ DFO treated and co-cultured. (D) Strategy used for sorting the MPB-10 and MPB-09 cells apart. CD235a + cells were used to determine where erythroblasts were in SSC/FSC, but not included in the sort strategy itself (dotted line). (E) Plots showing purity of samples collected from the co-cultures for both MPB-10 and MPB-09 cells for each condition from 1000 cells tested. An example of the strategy used for purity assessment. $(n=3)$ 


\subsection{Discussion}

Transfer of mitochondria between donor and recipient cells are most often shown through situations where cells have been stressed or injured $42,56-58,60-63,66$. This chapter shows that mtDNA transfer has not taken place to a detectable level between a Deferoxamine-treated and an untreated erythroblast population in co-culture. The limit of detection is considerably higher $(0.1 \%)$ than the cell line work shown previously (0.01-0.001\%). Furthermore, while deferoxamine can induce early apoptosis after 24hour treatments in these cells at the dose chosen, this injury may not require mitochondrial transfer to recover.

Erythroblasts are located in the bone marrow and can be obtained through liquid biopsies of bone marrow aspirates73,88. An alternative method for culturing erythroblasts from peripheral blood mononuclear cells was previously published in 2010, which was successfully repeated in this thesis87. This culturing method produced a mixed culture beginning with fairly equal proportions of erythroblasts, macrophages, and lymphocytes. Published research showed the erythroid population grew from $7 \%$ at day 3 , to $49 \%$ at day 7 , to $86 \%$ at day 13 and peaked around $90 \%$ at day 17 of culture 87 . Over time my cultures changed composition at similar rates and reached $80 \%$ erythroblasts with remaining cells displaying macrophage phenotypes (Fig $5.1 \& 5.2$ ). It is also important to note that expression of common surface markers and morphological phenotypes were observed, indicating that these cells were a suitable model to simulate bone marrow erythroblasts ex vivo (Fig 5.1 \& 5.2)79,87. Interestingly, these cultures also provided a method to monitor the differentiation process for erythroblasts over the 17day time period using these markers79. In future, this would allow for experiments investigating mitochondrial transfer between erythroblasts at many different stages of erythropoiesis from pro-erythroblasts to polychromatophilic erythroblasts.

Evidence of mitochondrial transfer is most commonly associated by formation of tunnelling nanotubules between cells of an adherent nature 53,55,56,58,59,62,63,66. Intriguingly, cytoplasmic bridges which resemble these tunnelling nanotubules can be seen in clinical bone marrow aspirates between $0.5-5 \%$ of erythroblasts 81,82 . Evidence suggests they are most commonly seen between polychromatophilic and orthochromatophilic staged erythroblasts (personal communication, Andrew Hall, MIMR, 2016)128. Inter-erythroblast bridges were identified in my cultures from PBMCs 
at low levels using brightfield microscopy, however these were difficult to image and did not last through processing onto slides for visualisation (Fig 5.1). Clarity regarding cytoplasmic bridge formation is lacking, therefore they could merely be remnants of cell division, as opposed to actively formed connections. Newly published data supporting mitochondrial transfer in bone marrow, albeit between human multiple myeloma cells and adherent human bone marrow stromal cells, shows that CD38 may be involved in the formation of tunnelling nanotubules between these cell types63. CD38 is a multifunctional membrane spanning enzyme involved in cell-cell adhesion, cytokine production and even T-cell co-stimulation, and is expressed on immune cells such as B-, T- and natural killer cells and also on erythroblasts63,129. The cell surface expression data of the erythroid cells from the PBMC culture supports previously published expression panels that CD38 is mostly expressed on pro-erythroblasts (Fig 5.2), however this stage of erythroid differentiation is not where majority of intererythroid bridges are seen in bone marrow erythroblasts(9, Personal Communication, Andrew Hall 2016). There may still be a role for CD38 in mitochondrial transfer within wider bone marrow contexts. However, the current data does not support a role for CD38 within mitochondrial transfer between erythroid cells.

Deferoxamine is a chemical that is primarily used clinically to chelate external iron as treatment for iron overload disorders50. While iron overload has negative effects on differentiation and survival of erythroid cells, so too does iron deprivation such as in anaemia75,77. Iron's importance in erythroid differentiation is also related to oxygen uptake through production of heme and thus haemoglobin in red blood cells, and mitochondrial function via the iron-sulfur clusters required for mitochondrial enzymes such as aconitase 120 . The erythroid cells cultured from PBMCs stained positively for presence of iron, generally located around the nucleus (Fig 5.1). Deferoxamine was chosen to induce injury for erythroblasts going into co-culture, based on the hypothesis that by removing any iron will impact mitochondrial function and reduce viability of the erythroid cells in the cultures.

Due to ethical and time constraints within this project, a complete dose-response titration, resembling that done in the cell line work previously, was not possible. However, data showed that $10-100 \mu \mathrm{M}$ deferoxamine was able to induce early apoptosis after 24 hours (Fig 5.4). Unfortunately, this panel did not have the ability to provide 
information about the membrane potential of the mitochondria in the treated cells. This is where previous data showed more of an effect at this 48 -hour time point with $25 \mu \mathrm{M}$ DFO treatment and could be investigated more in future. A reduction in mitochondrial membrane potential was still seen after a 48 -hour recovery period from $25 \mu \mathrm{M}$ deferoxamine (Fig 5.5). Results also showed that in the mixed culture, macrophages appeared to absorb more damage that was unable to be rectified (Fig 5.5). Macrophages are highly important to iron homeostasis through recycling in the human body and also provide support to erythroblasts natively in bone marrow through formation of erythroblast islands130. The culture method does not support growth of these macrophages thus proper nutrients required for macrophages to recover may not be present. Alternatively, if this native relationship still functions in these cultures, macrophages may have therefore sacrificed their own recovery to aid the erythroid cells

The chosen drug concentration of deferoxamine was unable to produce a window where assessment of the effect of co-culture on viability of treated cells could be analysed. This shows in the lack of difference between the $25 \mu \mathrm{M}$ treated, untreated and recovery model viability after co-culture (Fig 5.6). This was not unexpected - the preliminary titration data showed a lack of difference in apoptosis between the treated and untreated erythroblasts in the recovery model without co-culture (Fig 5.5). The co-culture models were initially designed to be kept consistent between in vitro and ex vivo work, given the time constraint on the project. However, further titrations may have shown that PBMC-derived erythroblasts required extended treatment to see effects on survival and even erythropoiesis from deferoxamine. Iron overload has been shown to alter the erythroid composition by increasing the proportion of immature erythroid cells and decreasing mature erythroid cells in both mice and in humans77. It is likely that this is due to increased apoptosis and ROS signalling in erythroid cells which prevents their differentiation through erythropoiesis and these responses are rescued by use of deferoxamine77. Alternatively, iron deprivation has also been shown to negatively impact erythroid cells. Low levels of iron such as that seen in anaemias increases erythropoietin resistance in erythroid cells leading their lack of early differentiation and survival75. A published study hypothesised that this may be due low levels of exogenous and endogenous iron reducing expression of the EPO-receptor on the cell surface via over-degradation of a protein called Scribble thus preventing adequate signalling from EPO75. Erythroid cells, when iron levels are low, therefore undergo iron restricted 
erythropoiesis74. Iron consequently has a delicate role in the health, differentiation and function of erythroid cells. Further experiments could leave the treatments and cocultures longer and assess the differentiation of the erythroblasts and whether mitochondrial transfer is required to rescue a potential phenotype of halted erythropoiesis.

Contrary to the cell line data presented earlier, in a primary cell population the allelespecific-blocker assay has a higher limit of detection $(0.1 \%)$ for polymorphisms in the mitochondrial genome, thus increasing the threshold of transfer required to be detectable. This was not unexpected due to the known presence of heteroplasmy within primary human mitochondrial genomes22,127,131-133. Heteroplasmy occurs at both a population and an individual level. Mitochondrial genomes can be used to trace geographical and familial (maternal) lineages where certain combinations of polymorphisms have been termed haplotypes 133,134. At the individual level, using early techniques, detection of point mutations indicated that healthy individuals could have less than $1 \%$ variation in their collective mitochondrial genomes at a given time 127. However, through the advancement of sequencing and DNA technologies, data has been published showing point mutations as infrequent as $0.2 \%$ within individuals 127 . With $0.2 \%$ natural heteroplasmy within a control population, a detection level of $0.1 \%$ using the allele-specific-blocker assay is consistent with this limitation, and supports the idea that that a few naturally occurring "target" mtDNA genomes are present in the control populations. This makes it harder to detect mitochondrial genome transfer if it is occurring at a low frequency into these heteroplasmic populations.

Interestingly, this limit of detection appeared to absorb the small levels of contamination from flow-based separation of the populations after co-culture. This means that although true mtDNA transfer events need to occur at higher rates to be detected by this assay, the results are unlikely to be swayed by low levels of contamination. To help quantify any contamination present, ideally, a nuclear control would also be designed to leverage the Y chromosome in MPB-09, however this was not completed for this thesis.

Techniques that allow a complete profile of the mitochondrial genomes present within an off-target cell population will provide more scope to detect deviations from these profiles possibly due to mitochondrial transfer. Examples of these techniques include 
single cell sequencing and assessing the collection of reads rather than generating a consensus sequence or using an in-situ PCR method called rolling circle amplification specific to multiple polymorphisms and pairing this to a computer-based quantitation software20,69,70.

Ultimately, this chapter showed that mtDNA transfer did not occur at a detectable level - i.e. more than $0.1 \%$ - between erythroblasts (Fig 5.7). This could be due to a number of reasons including the lack of CD38 expressed at Day 10 of culture (where the coculture experiments took place) which could lead to inability to form inter-erythroid bridges. Alternatively, the cells were not stressed enough to induce transfer - the drug treatment lacked the severity required. This could be overcome with further experiments at higher concentrations. Additionally, the limitations of the qPCR technique used to detect the transfer could also affect the results.

Future directions with this research could include other treatments could also be explored such as antibiotics that affect mitochondria, that are commonly used after bone marrow transplants, or increasing iron in the culture media to simulate iron overload. However, there is still the ultimate possibility that erythroblasts cannot donate mitochondria to each other and instead require the presence of a more common donor such as a bone marrow stromal cell to undergo transfer of mitochondria.

\subsection{Conclusion}

To translate cell line in vitro mitochondrial transfer experiments closer to in vivo work, a culture method to produce ex vivo erythroblasts from PBMCs was replicated from previously published data87. mtDNA polymorphisms between donors of PBMCs were identified and leveraged to develop an allele specific qPCR assay for the detection of mitochondrial DNA transfer. A drug treatment was selected to induce an injury to erythroblast cells to promote transfer of mitochondria when in co-culture conditions. However, mtDNA transfer was not detected between these erythroblast cell populations using the techniques in this chapter. 
6 Final Discussion 


\subsection{Discussion}

This thesis aimed to explore transfer of mitochondria between human erythroblasts. Mitochondrial transfer was explored using a po cell model in vitro, a drug induced model in vitro using deferoxamine and cisplatin, and finally ex vivo using erythroblasts differentiated from PBMCs treated with deferoxamine. The techniques used were ultimately unable to detect any transfer of mitochondria in all three conditions after coculture with healthy cells, however co-culture was able to improve recovery.

Visual microscopic techniques are most commonly used to detect transfer of mitochondria in published research 42,54,56-59,63, however these techniques have limitations 67,68 . Visual methods such as dyes contained in mitochondria by membrane potential can be released due to fluctuations in membrane potential 67,68. Reduction in membrane potential from drug treatments would therefore prevent these cells from being stained which only permits unidirectional detection of mitochondrial transfer. The results from drug titrations in this thesis of cisplatin, in cell lines, deferoxamine in cell lines and primary erythroblasts, indicated a clear reduction in mitochondrial potential as a measure of mitochondrial injury (Fig 4.3, 4.4, 4.8, 4.9, 5.4). Therefore, visual methods would not be suitable for analysing unbiased transfer of mitochondria in co-culture systems when investigating the impact of treatments, limiting this thesis and similarly published research.

Alternate methods of mitochondrial transfer focus on mtDNA 68. Molecular methods rely on presence of sequence differences between the donor and recipient cells 39,61-64. Sanger sequencing, and interspecies end point PCRs can be useful in the right conditions, as previously published 39,61-64. However, higher sensitivity was desired for experiments in this thesis. An allele-specific-blocker qPCR assay was previously designed within our lab group that could distinguish $0.01 \%-0.001 \%$ HEL mtDNA from off-target K562 and HL-60 mtDNA, a very high level of sensitivity. This assay functions by selectively amplifying target mtDNA, and blocking amplification of offtarget mtDNA, using a single polymorphic difference, and detected by fluorescent probe 69. The sensitivity of these assays is limited by the amount of noise created in the reactions by mis-match amplification of the off-target mtDNA polymorphisms which can be increased depending on the specific nucleotide differences 69 . For this thesis, modifications to this assay allowed detection of HL-60 mtDNA using two 
polymorphisms separated by a single nucleotide at the same sensitivity, $0.001 \%$ target mtDNA. Therefore, providing a highly sensitive molecular assay to identify rare mitochondrial transfer events within the experiments conducted in this thesis.

To determine target mtDNA presence after co-culture using these ASBqPCR assays, cells needed to be separated using fluorescence activated cell sorting (FACS) into individual populations. The level of sensitivity of the ASBqPCR can detect very rare transfer events, therefore requiring $100 \%$ pure off-target DNA samples with no contaminating target cells. One target cell contains multiple copies of target mtDNA $19,22,135$, which can skew the amplification and lead to false positives. FACS separating injured cells from co-culture, both the HL-60 po cells and deferoxamine or cisplatin treated HEL cells, proved difficult to obtain the level of purity required with minimal success. Alterations to gating strategies and sorting each population twice increased purities to above $98 \%$ however, this was not sufficient to prevent contamination skewing the ASBqPCR data. Ultimately, this prevented detection of mtDNA between deprived HL-60 0 o and HEL cells, or HEL cells, treated with either DFO or cisplatin, and HL-60 cells as concluded in chapters 3 and 4 of this thesis. Interestingly, in po and drug treated cell line in vitro work, the FACS separation was the limiting technique rather than the molecular ASBqPCR technique. Therefore, the results could not exclude that it did happen in the co-culture systems for either condition.

Nuclear controls can help quantify the level of contamination in these samples 19. Potentially enabling higher resolution of mtDNA transfer for the co-culture samples tested by ASBqPCR in chapters 3 and 4. Throughout the course of this thesis, nuclear controls in the form of short tandem repeats (STR), and Y chromosome qPCR were explored for the cell lines. The cell lines did contain short tandem repeat differences between them however, resolving these differences using end point PCR and obtaining sufficient sensitivity was not successful (data not shown). Y chromosome qPCR was next investigated as this would allow detection of HEL cell contamination, however, upon XY FISH investigation conducted by the hospital on my cell lines, HEL cells no longer maintained their Y chromosome (data not shown).

Another ASBqPCR assay was developed to detect peripheral blood donor MPB-09 mtDNA over background MPB-10 mtDNA. This assay was 10-100-fold less sensitive compared to the cell line specific assays, $0.1 \%$ compared to the previous $0.01 \%$ - 
$0.001 \%$. This was not unexpected due to the presence of heteroplasmy of mitochondrial genomes in human primary cells 20-22,131,132. Heteroplasmy causes the off-target DNA sample to amplify earlier in the reaction because a few target mitochondrial genomes are present. Previously believed to be around 1\%, newer techniques now estimate heteroplasmy in polymorphisms to occur in $0.2 \%$ of mitochondrial genomes 21,132 . Therefore, a $0.1 \%$ sensitivity for detection of basal heteroplasmy is concurrent with published estimates indicating that the assay can still detect mtDNA transfer above this heteroplasmic level.

Throughout these experiments it became apparent that sorting primary human cells was easier than sorting the cell lines and achieved overall higher purity. Interestingly, the final qPCR analysis of separated populations did not show a difference in amplification between HEL samples that were $99.8 \%$ pure and 95\% pure. Regardless of purity, all samples amplified at the same cycle as the reference sample indicating that the heteroplasmic limit of the assay can absorb the contaminating cells in a way the cell line ASBqPCR assays could not. Therefore, mitochondrial transfer was still able to be detected in these conditions if at a level above the $0.1 \%$ sensitivity. However, in the cocultures performed in this thesis, mitochondrial transfer did not occur at a detectable level, above the heteroplasmic background.

Manipulations of FACS gating strategy to maximise purity may have biased the ability to detect mtDNA transfer by ASBqPCR in the co-cultures between HEL and HL-60 cells. Including damaged and dying cells in the sort strategy, increased fanning of sorting streams thereby correlating to increased contamination. To reduce this, gating required cells included to be at their healthiest possible state. An assumption required by these modifications, is that cells that took up mitochondria from the healthy donor were fully recovered by 48 hours. This is a small window for cells to have obtained mitochondria and for those mitochondria to replicate and propagate within recipient cells. Transfer of mitochondria has been previously shown to occur within 24 hours of co-culture in vitro 57 and the mitochondria slowly increase in proportion under the right conditions time dependently which could take 3 days or more 59. These results show that depending on the injury and the conditions, complete recovery from mitochondrial transfer may take longer than the 48 hours tested in this thesis, therefore cells that may 
have taken up mitochondria were likely still contained within the injured cell populations, not included by FACS and unable to be analysed by ASBqPCR.

This thesis showed deprived HL-60 po cells in co-culture were protected from damage, and deferoxamine treated HEL cells improved recovery when in co-culture.

Interestingly, for DFO treated HEL cells, the recovery effect increased further when the live cell gating strategy included intermediately stained cells. Including these cells in analysis, implies an interesting assumption. That slightly permeable cells may be capable, or in the process of, reversing cell death. The ability of cells to reverse the apoptotic state is termed anastasis and is most commonly seen in transient injuries 108,109, for example deferoxamine treatment for 24 hours and then removed as performed in this thesis. Cells have been shown to recover even from the committed state of caspase activation in apoptosis 108,109. From my results, co-culture may play a role in speeding up anastasis as shown by increased recovery within 48 hours, compared to HEL cells that recovered without co-culture. Trophic support provided by healthy cells within co-culture system are likely drivers of this result 96-98,104,136. Subsequently, relation of transfer of mitochondria to this trophic support was not determined due to the technical limitations of this thesis. My results also support that some injuries can be too severe to recover from within 48 hours, as seen with cisplatin treated HEL cells.

Improved recovery from co-culture was not observed between ex vivo erythroblast cells in this thesis. This was likely due to an incorrectly chosen concentration of deferoxamine. Complete 96-hour time courses of deferoxamine and cisplatin titrations were completed on HEL and K562 cells in preparation for co-cultures to identify mitochondrial transfer. However, ethical limitations such as limited blood volumes and donor frequency, and most importantly, time, prevented the same experiments from being conducted on ex vivo PBMC-differentiated erythroblasts prior to co-culture. Therefore, a concentration that induced sufficient injury in the cells was not chosen causing lack of resolution into effect of co-culture for these cells and should be corrected in future research.

Erythroblasts have not been studied previously for mitochondrial transfer. Additionally, research focusses on the assumption that a certain cell type, likely mesenchymal, stromal and/or fibroblast like, is only capable of donating mitochondria 42,53,62,63,54-61. However, while there is data proving that these cell types can transfer mitochondria, 
there is a lack of data showing that other cells cannot. Most likely due to the difficult nature of publishing negative data. Therefore, this thesis had the potential to provide some data for a new cell type, erythroblasts, within the mitochondrial transfer field and explore the possibility that transfer may be possible between two erythroblasts, rather than relying on a mesenchymal, stromal or fibroblast type cell.

CD38 has been previously implicated in transfer of mitochondria, including within bone marrow context 63,107. Therefore, CD38 expression on erythroblasts was an initial indicator, alongside their generation of potential cytoplasmic bridges 82 , that transfer of mitochondria was possible between these cells. Previous research in our lab group characterised the formation of inter-erythroid bridges to between polychromatophilic and orthochromatic erythroblast stages (personal communication, Andrew Hall, MIMR, 2016). These bridges were also replicated between the cultured erythroid cells derived ex vivo from PBMCs. However, the results of this thesis, supporting previous research indicates that CD38 is not co-expressed with inter-erythroid bridges, rather at the earlier pro-erythroblast stage 79 . Therefore, a link between CD38 expression and inter-erythroid bridges was not supported by this thesis.

The greater question this thesis aimed to shed light on, was the possibility of erythroid cells to donate mitochondria to each other. A concept not proven in previous research. Due to techniques used in this thesis, mitochondrial transfer via molecular mtDNA read out was not detectable after FACS separation of co-cultured populations. Therefore, I could not conclude that mtDNA transfer occurred. Conversely, I cannot conclude, definitively, that mtDNA transfer did not occur at all. This question is still an important question to answer for the field of mitochondrial transfer, not only between erythroblasts but for the wider field in all contexts. Future research should focus on definitively answering this question. 


\subsection{Future Directions}

To overcome the limitations encountered in this thesis, namely contamination from FACS skewing the ASBqPCR mtDNA detection, another method of mtDNA detection should be investigated. I propose that future research employs the use of a technique combining visual and molecular methods. mtDNA polymorphisms can be leveraged by using an allele-specific padlock probe 70. Cell no longer need to be FACS separated for DNA analysis by using this method because distinguishing features can be identified by the microscopy, such as using the same proliferation dyes used in this thesis. Subsequently, the padlock probes in each of these cells are PCR amplified on the microscope slides using rolling circle amplification 70. DNA binding sites on the probe lead to dimerization of the amplified padlock and can be linked together using repeat regions to generate discrete PCR products 70 . These PCR products have sequences repeated that are specific to the mtDNA polymorphism and are bound by different fluorescent probes that are excited using confocal microscopy 70. This technique also allows basal heteroplasmy determination, making it useful for primary human analysis. When paired with high throughput imaging and machine learning technology, this technique could determine mitochondrial genome transfer events above heteroplasmic or technique limited false positives. 


\section{References}

1. Jonckheere, A. I., Smeitink, J. A. M. \& Rodenburg, R. J. T. Mitochondrial ATP synthase: architecture, function and pathology. J. Inherit. Metab. Dis. $35,211-25$ (2012).

2. Li, M. X. \& Dewson, G. Mitochondria and apoptosis: emerging concepts. F1000Prime Rep. 7, 42 (2015).

3. Wlodkowic, D., Telford, W., Skommer, J. \& Darzynkiewicz, Z. Apoptosis and Beyond: Cytometry in Studies of Programmed Cell Death. doi:10.1016/B978-0-12-385493-3.00004-8

4. Frey, T. G. \& Mannella, C. A. The internal structure of mitochondria. Trends Biochem. Sci. 25, 319-24 (2000).

5. Rafelski, S. M. Mitochondrial network morphology: building an integrative, geometrical view. BMC Biol. 11, 71 (2013).

6. van der Bliek, A., Shen, Q. \& Kawajiri, S. Mechanisms of mitochondrial fission and fusion. Cold Spring Harb Perspect Bio/ 5, 1-16 (2013).

7. Youle, R. J. et al. REVIEW Mitochondrial Fission, Fusion, and Stress. Science (80-. ). 337, 1062-1065 (2012).

8. Lee, H.-C., Yin, P.-H., Chi, C.-W. \& Wei, Y.-H. Increase in Mitochondrial Mass in Human Fibroblasts under Oxidative Stress and during Replicative Cell Senescence. J. Biomed. Sci. 9, 517-526 (2002).

9. Gray, M. W. et al. Mitochondrial Evolution. Spring 1-16 (2012). doi:10.1101/cshperspect.a011403

10. Martin, W. F., Garg, S. \& Zimorski, V. Endosymbiotic theories for eukaryote origin. Philos. Trans. R. Soc. B Biol. Sci. 370, 20140330 (2015).

11. Gross, J. \& Bhattacharya, D. Endosymbiont or host: Who drove mitochondrial and plastid evolution? Biol. Direct 6, 12 (2011).

12. Taanman, J.-W. The mitochondrial genome: structure, transcription, translation and replication. Biochim. Biophys. Acta - Bioenerg. 1410, 103- 
123 (1999).

13. Anderson, S. et al. Sequence and organization of the human mitochondrial genome. Nature 290, 457-465 (1981).

14. Chomyn, A. et al. Six unidentified reading frames of human mitochondrial DNA encode components of the respiratory-chain NADH dehydrogenase. Nature 314, 592-597 (1985).

15. Chomyn, A. et al. URF6, last unidentified reading frame of human mtDNA, codes for an NADH dehydrogenase subunit. Science (80-. ). 234, 614-618 (1986).

16. Macreadie, I. G. et al. Biogenesis of mitochondria: the mitochondrial gene ( aap 1) coding for mitochondrial ATPase subunit 8 in Saccharomyces cerevisiae. Nucleic Acids Res. 11, 4435-4451 (1983).

17. Holt, I. J. \& Reyes, A. Human mitochondrial DNA replication. Cold Spring Harb. Perspect. Biol. 4, (2012).

18. Ramos, A. et al. Frequency and Pattern of Heteroplasmy in the Complete Human Mitochondrial Genome. PLoS One 8, e74636 (2013).

19. Cagnone, G. et al. Analysis of the Mitochondrial DNA and Its Replicative Capacity in Induced Pluripotent Stem Cells. in 231-267 (Humana Press, New York, NY, 2014). doi:10.1007/7651_2014_156

20. Yao, Y.-G., Kajigaya, S. \& Young, N. S. Mitochondrial DNA mutations in single human blood cells. Mutat. Res. Mol. Mech. Mutagen. 779, 68-77 (2015).

21. Stewart, J. B. \& Chinnery, P. F. The dynamics of mitochondrial DNA heteroplasmy: implications for human health and disease. Nat. Rev. Genet. $16,530-542$ (2015).

22. Lightowlers, R. N., Chinnery, P. F., Turnbull, D. M. \& Howell, N. Mammalian mitochondrial genetics: heredity, heteroplasmy and disease. Trends Genet. 13, 450-455 (1997).

23. Choudhury, A. R. \& Tiwari, H. K. Numtogenesis as a mechanism for 
development of cancer. Semin. Cancer Biol. 47, 101-109 (2017).

24. Hazkani-Covo, E., Zeller, R. M. \& Martin, W. Molecular Poltergeists:

Mitochondrial DNA Copies (numts) in Sequenced Nuclear Genomes. PLOS Genet. 6, e1000834 (2010).

25. Lodish, H. et al. Electron Transport and Oxidative Phosphorylation. (2000).

26. Bajzikova, M. et al. Reactivation of Dihydroorotate Dehydrogenase-Driven Pyrimidine Biosynthesis Restores Tumor Growth of Respiration-Deficient Cancer Cells. Cell Metab. (2018). doi:10.1016/j.cmet.2018.10.014

27. Formosa, L. E., Dibley, M. G. \& Stroud, D. A. Building a complex complex: Assembly of mitochondrial respiratory chain complex I. Semin. Cell Dev. Biol. 76, 154-162 (2018).

28. St John, J. C., Lloyd, R. E. I., Bowles, E. J., Thomas, E. C. \& El Shourbagy, S. The consequences of nuclear transfer for mammalian foetal development and offspring survival. A mitochondrial DNA perspective. Reproduction $127,631-641$ (2004).

29. Tan, A. S., Baty, J. W. \& Berridge, M. V. The role of mitochondrial electron transport in tumorigenesis and metastasis. Biochim. Biophys. Acta - Gen. Subj. 1840, 1454-1463 (2014).

30. Zorov, D. B., Juhaszova, M. \& Sollott, S. J. Mitochondrial Reactive Oxygen Species (ROS) and ROS-Induced ROS Release. Physiol. Rev. 94, 909-950 (2014).

31. Brandon, M., Baldi, P. \& Wallace, D. C. Mitochondrial mutations in cancer. Oncogene 25, 4647-4662 (2006).

32. Ahn, C. S. \& Metallo, C. M. Mitochondria as biosynthetic factories for cancer proliferation. Cancer Metab. 3, 1 (2015).

33. Waldvogel-Abramowski, S. et al. Physiology of iron metabolism. Transfus. Med. Hemother. 41, 213-21 (2014).

34. Atamna, H., Walter, P. B. \& Ames, B. N. The Role of Heme and Iron-Sulfur Clusters in Mitochondrial Biogenesis, Maintenance, and Decay with Age. 
Arch. Biochem. Biophys. 397, 345-353 (2002).

35. Zhang, L. \& Guarente, L. Heme binds to a short sequence that serves a regulatory function in diverse proteins. EMBO J. 14, 313-20 (1995).

36. Falk, M. J. \& Sondheimer, N. Mitochondrial genetic diseases. Curr. Opin. Pediatr. 22, 711-6 (2010).

37. Vander Heiden, M. G., Cantley, L. C. \& Thompson, C. B. Understanding the Warburg effect: the metabolic requirements of cell proliferation. Science 324, 1029-33 (2009).

38. Berridge, M. V., Crasso, C. \& Neuzil, J. Mitochondrial genome transfer to tumour cells breaks the rules and establishes a new precedent in cancer biology. Mol. Cell. Oncol. 00-00 (2015). doi:10.1080/23723556.2015.1023929

39. Tan, A. S. et al. Mitochondrial genome acquisition restores respiratory function and tumorigenic potential of cancer cells without mitochondrial DNA. Cell Metab 21, 81-94 (2015).

40. Kukat, A. et al. Generation of rho0 cells utilizing a mitochondrially targeted restriction endonuclease and comparative analyses. Nucleic Acids Res. 36, e44 (2008).

41. Berridge, M. V. et al. Horizontal transfer of mitochondria between mammalian cells: Beyond co-culture approaches. Curr. Opin. Genet. Dev. $38,75-82$ (2016).

42. Spees, J. L., Olson, S. D., Whitney, M. J. \& Prockop, D. J. Mitochondrial transfer between cells can rescue aerobic respiration. Proc Natl Acad Sci U $S A 103,1283-1288$ (2006).

43. Herst, P. M., Levine, D. M. \& Berridge, M. V. Mitochondrial gene knockout HL60 0 cells show preferential differentiation into monocytes/macrophages. Leuk. Res. 29, 1163-1170 (2005).

44. Yakes, F. M. \& Van Houten, B. Mitochondrial DNA damage is more extensive and persists longer than nuclear DNA damage in human cells 
following oxidative stress. Proc. Natl. Acad. Sci. U. S. A. 94, 514-9 (1997).

45. Atamna, H., Liu, J. \& Ames, B. N. Heme deficiency selectively interrupts assembly of mitochondrial complex IV in human fibroblasts: revelance to aging. J. Biol. Chem. 276, 48410-6 (2001).

46. Zhang, Z. W. et al. Red blood cell extrudes nucleus and mitochondria against oxidative stress. IUBMB Life 63, 560-565 (2011).

47. Atamna, H. Heme, iron, and the mitochondrial decay of ageing. Ageing Res. Rev. 3, 303-18 (2004).

48. Hatcher, H. C., Singh, R. N., Torti, F. M. \& Torti, S. V. Synthetic and natural iron chelators: therapeutic potential and clinical use. Future Med. Chem. 1, 1643-1670 (2009).

49. Westlin, W. F. Deferoxamine as a Chelating Agent. Clin. Toxicol. 4, 597-602 (1971).

50. Bentur, Y., McGuigan, M. \& Koren, G. Deferoxamine (Desferrioxamine) New Toxicities for an Old Drug. Drug Saf. 6, 37-46 (1991).

51. Dasari, S. \& Tchounwou, P. B. Cisplatin in cancer therapy: molecular mechanisms of action. Eur. J. Pharmacol. 740, 364-78 (2014).

52. Rustom, A., Saffrich, R., Markovic, I., Walther, P. \& Gerdes, H. H. Nanotubular Highways for Intercellular Organelle Transport. Science (80-. ). 303, 1007-1010 (2004).

53. Spees, J. L., Lee, R. H. \& Gregory, C. A. Mechanisms of mesenchymal stem/stromal cell function. Stem Cell Res. Ther. 7, 125 (2016).

54. Phinney, D. G. et al. Mesenchymal stem cells use extracellular vesicles to outsource mitophagy and shuttle microRNAs. Nat. Commun. 6, 1-15 (2015).

55. Ahmad, T. et al. Miro1 regulates intercellular mitochondrial transport \& enhances mesenchymal stem cell rescue efficacy. EMBO J33, 994-1010 (2014).

56. Jiang, D. et al. Mitochondrial transfer of mesenchymal stem cells 
effectively protects corneal epithelial cells from mitochondrial damage. Cell Death Dis. 7, e2467-10 (2016).

57. Boukelmoune, N., Chiu, G. S., Kavelaars, A. \& Heijnen, C. J. Mitochondrial transfer from mesenchymal stem cells to neural stem cells protects against the neurotoxic effects of cisplatin. Acta Neuropathol. Commun. 6, 139 (2018).

58. Babenko, V. et al. Miro1 Enhances Mitochondria Transfer from Multipotent Mesenchymal Stem Cells (MMSC) to Neural Cells and Improves the Efficacy of Cell Recovery. Molecules 23, 687 (2018).

59. Wang, J. et al. Cell adhesion-mediated mitochondria transfer contributes to mesenchymal stem cell-induced chemoresistance on T cell acute lymphoblastic leukemia cells. J. Hematol. Oncol. 11, 11 (2018).

60. Islam, M. N. et al. Mitochondrial transfer from bone-marrow-derived stromal cells to pulmonary alveoli protects against acute lung injury. Nat Med 18, 759-765 (2012).

61. Moschoi, R. et al. Protective mitochondrial transfer from bone marrow stromal cells to acute myeloid leukemic cells during chemotherapy. 128, 253-265 (2017).

62. Marlein, C. R. et al. NADPH oxidase-2 derived superoxide drives mitochondrial transfer from bone marrow stromal cells to leukemic blasts. Blood 130, 1649-1660 (2017).

63. Marlein, C. R. et al. CD38-driven mitochondrial trafficking promotes bioenergetic plasticity in multiple myeloma. Cancer Res. canres.0773.2018 (2019). doi:10.1158/0008-5472.CAN-18-0773

64. Dong, L. F. et al. Horizontal transfer of whole mitochondria restores tumorigenic potential in mitochondrial DNA-deficient cancer cells. Elife 6, 1-22 (2017).

65. Davis, C. O. et al. Transcellular degradation of axonal mitochondria. Proc. Natl. Acad. Sci. U. S. A. 111, 9633-8 (2014). 
66. Pasquier, J. et al. Preferential transfer of mitochondria from endothelial to cancer cells through tunneling nanotubes modulates chemoresistance. J. Transl. Med. 11, 1 (2013).

67. Perry, S. W., Norman, J. P., Barbieri, J., Brown, E. B. \& Gelbard, H. A. Mitochondrial membrane potential probes and the proton gradient: a practical usage guide. Biotechniques 50, 98-115 (2011).

68. Berridge, M. V., Herst, P. M., Rowe, M. R., Schneider, R. \& McConnell, M. J. Mitochondrial transfer between cells: Methodological constraints in cell culture and animal models. Anal. Biochem. 552, 75-80 (2018).

69. Morlan, J., Baker, J. \& Sinicropi, D. Mutation detection by real-time PCR: A simple, robust and highly selective method. PLoS One 4, (2009).

70. Krzywkowski, T., Hauling, T. \& Nilsson, M. In Situ Single-Molecule RNA Genotyping Using Padlock Probes and Rolling Circle Amplification. in Methods in molecular biology (Clifton, N.J.) 1492, 59-76 (2017).

71. Reagan, M. R. \& Rosen, C. J. Navigating the bone marrow niche: translational insights and cancer-driven dysfunction. Nat. Rev. Rheumatol. 12, 154-168 (2016).

72. Hoffman, R. et al. Hematology: basic principles and practice.

73. Moras, M., Lefevre, S. D. \& Ostuni, M. A. From Erythroblasts to Mature Red Blood Cells: Organelle Clearance in Mammals. Front. Physiol. 8, 1076 (2017).

74. Goodnough, L. T. Erythropoietin and iron-restricted erythropoiesis. Exp. Hematol. 35, 167-172 (2007).

75. Khalil, S. et al. Iron modulation of erythropoiesis is associated with Scribble-mediated control of the erythropoietin receptor. J. Exp. Med. 215, 661-679 (2018).

76. Liu, J., Han, X. \& An, X. Novel methods for studying normal and disordered erythropoiesis. Sci. China Life Sci. 58, 1270-1275 (2015).

77. Taoka, K. et al. The effect of iron overload and chelation on erythroid 
differentiation. Int. J. Hematol. 95, 149-159 (2012).

78. Weiss, G. Iron and anemia of chronic disease. Kidney Int. 55, S12-S17 (1999).

79. Fajtova, M., Kovarikova, A., Svec, P., Kankuri, E. \& Sedlak, J. Immunophenotypic profile of nucleated erythroid progenitors during maturation in regenerating bone marrow. Leuk. Lymphoma 54, 2523-2530 (2013).

80. Albenız, I., Türker-Şener, L., Baş, A., Kalelıoğlu, I. \& Nurten, R. Isolation of hematopoietic stem cells and the effect of CD38 expression during the early erythroid progenitor cell development process. Oncol. Lett. 3, 55-60 (2012).

81. Kobayashi, M., Asano, H. \& Hotta, T. [Occurrence of cytoplasmic bridge between erythroblasts--morphology and frequency in hematological disease]. Rinsho. Ketsueki. 31, 946-50 (1990).

82. WICKRAMASINGHE, S. N. DYSERYTHROPOIESIS AND CONGENITAL DYSERYTHROPOIETIC ANAEMIAS. Br. J. Haematol. 98, 785-797 (1997).

83. Martin, P. \& Papayannopoulou, T. HEL cells: a new human erythroleukemia cell line with spontaneous and induced globin expression. Science (80-. ). 216, 1233-1235 (1982).

84. Koeffler, H. P. \& Golde, D. W. Human myeloid leukemia cell lines: a review. Blood 56, 344-50 (1980).

85. Naumann, S., Reutzel, D., Speicher, M. \& Decker, H.-J. J. Complete karyotype characterization of the K562 cell line by combined application of G-banding, multiplex-fluorescence in situ hybridization, fluorescence in situ hybridization, and comparative genomic hybridization. Leuk. Res. 25, 313-322 (2001).

86. Birnie, G. D. The HL60 cell line: a model system for studying human myeloid cell differentiation. Br. J. Cancer. Suppl. 9, 41-5 (1988).

87. Migliaccio, G. et al. Humanized culture medium for clinical expansion of 
human erythroblasts. Cell Transplant. 19, 453-469 (2010).

88. Bain, B. J. Leaders Bone marrow aspiration. J Clin Pathol 54, (2001).

89. Sengsayadeth, S. et al. Reduced intensity conditioning allogeneic hematopoietic cell transplantation for adult acute myeloid leukemia in complete remission -a review from the acute leukemia working party of the EBMT. Haematologica 100, 859-869 (2015).

90. Bacigalupo, A. et al. Defining the intensity of conditioning regimens: working definitions. Biol. Blood Marrow Transplant. 15, 1628-33 (2009).

91. Wada, H. et al. Expression of major blood group antigens on human erythroid cells in a two phase liquid culture system. Blood 75, 505-11 (1990).

92. Vyas, S., Zaganjor, E. \& Haigis, M. C. Mitochondria and Cancer. Cel/ 166, 555-566 (2016).

93. Berridge, M. V. \& Neuzil, J. The mobility of mitochondria: Intercellular trafficking in health and disease. Clin. Exp. Pharmacol. Physiol. 44, 15-20 (2017).

94. Berridge, M. V \& Tan, A. S. Effects of mitochondrial gene deletion on tumorigenicity of metastatic melanoma: reassessing the Warburg effect. Rejuvenation Res. 13, 139-41 (2010).

95. de Oliveira, D. C. et al. Glutamine metabolism and its effects on immune response: molecular mechanism and gene expression. Nutrire 41, 14 (2016).

96. Suh, S. W., Aoyama, K., Matsumori, Y., Liu, J. \& Swanson, R. A. Pyruvate administered after severe hypoglycemia reduces neuronal death and cognitive impairment. Diabetes 54, 1452-8 (2005).

97. Selak, I., Skaper, S. D. \& Varon, S. Pyruvate participation in the low molecular weight trophic activity for central nervous system neurons in glia-conditioned media. J. Neurosci. 5, 23-8 (1985).

98. Fu, Y. et al. Trophic Effects of Mesenchymal Stem Cells in Tissue 
Regeneration. doi:10.1089/ten.teb.2016.0365

99. Cho, Y. M. et al. Mesenchymal stem cells transfer mitochondria to the cells with virtually no mitochondrial function but not with pathogenic mtDNA mutations. PLoS One 7, e32778 (2012).

100. Arsenic, R. et al. Comparison of targeted next-generation sequencing and Sanger sequencing for the detection of PIK3CA mutations in breast cancer. BMC Clin. Pathol. 15, 20 (2015).

101. Campbell, N. A. et al. Biology. (Pearson Benjamin Cummings, 2009).

102. Lee, R. H. et al. Multipotent stromal cells from human marrow home to and promote repair of pancreatic islets and renal glomeruli in diabetic NOD/scid mice. Proc Natl Acad Sci U S A 103, (2006).

103. Berridge, M. V., Schneider, R. T. \& McConnell, M. J. Mitochondrial Transfer from Astrocytes to Neurons following Ischemic Insult: Guilt by Association? Cell Metab. 24, 376-378 (2016).

104. Pleumeekers, M. M. et al. Trophic effects of adipose-tissue-derived and bone-marrow-derived mesenchymal stem cells enhance cartilage generation by chondrocytes in co-culture. PLoS One 13, e0190744 (2018).

105. Herst, P. M., Rowe, M. R., Carson, G. M. \& Berridge, M. V. Functional Mitochondria in Health and Disease. Front. Endocrinol. (Lausanne). 8, 296 (2017).

106. Accardi, F. et al. Mechanism of Action of Bortezomib and the New Proteasome Inhibitors on Myeloma Cells and the Bone Microenvironment: Impact on Myeloma-Induced Alterations of Bone Remodeling. Biomed Res. Int. 2015, 1-13 (2015).

107. Hayakawa, K. et al. Transfer of mitochondria from astrocytes to neurons after stroke. Nature 535, 551-555 (2016).

108. Sun, G. et al. A molecular signature for anastasis, recovery from the brink of apoptotic cell death. J. Cell Biol. 216, 3355-3368 (2017).

109. Tang, H. L., Yuen, K. L., Tang, H. M. \& Fung, M. C. Reversibility of apoptosis 
in cancer cells. Br. J. Cancer 100, 118-22 (2009).

110. Dean, A., Erard, F., Schneider, A. P. \& Schechter, A. N. Induction of hemoglobin accumulation in human $\mathrm{K} 562$ cells by hemin is reversible. Science 212, 459-61 (1981).

111. Tait, S. W. G., Ichim, G. \& Green, D. R. Die another way--non-apoptotic mechanisms of cell death. J. Cell Sci. 127, 2135-44 (2014).

112. Brady, S. T., Siegel, G. J., Albers, R. W. (Robert W. \& Price, D. L. (Donald L. Basic neurochemistry: principles of molecular, cellular, and medical neurobiology. (2012).

113. Bianchi, N. et al. Induction of erythroid differentiation of human K562 cells by cisplatin analogs. Biochem. Pharmacol. 60, 31-40 (2000).

114. Shariati, L. et al. Comparison of different methods for erythroid differentiation in the K562 cell line. Biotechnol. Lett. 38, 1243-1250 (2016).

115. Koh, B., Jeon, H., Kim, D., Kang, D. \& Kim, K. R. Effect of fibroblast coculture on the proliferation, viability and drug response of colon cancer cells. Oncol. Lett. 17, 2409-2417 (2019).

116. Majety, M., Pradel, L. P., Gies, M. \& Ries, C. H. Fibroblasts Influence Survival and Therapeutic Response in a 3D Co-Culture Model. PLoS One 10, e0127948 (2015).

117. Yuan, X., Wang, X., Chen, C., Zhou, J. \& Han, M. Bone mesenchymal stem cells ameliorate ischemia/reperfusion-induced damage in renal epithelial cells via microRNA-223. Stem Cell Res. Ther. 8, 146 (2017).

118. Fitzpatrick, E. et al. Coculture with Mesenchymal Stem Cells Results in Improved Viability and Function of Human Hepatocytes. Cell Transplant. 24, 73-83 (2015).

119. Luo, S. et al. Biparental Inheritance of Mitochondrial DNA in Humans. doi:10.1073/pnas.1810946115

120. Atamna, H., Walter, P. B. \& Ames, B. N. The role of heme and iron-sulfur clusters in mitochondrial biogenesis, maintenance, and decay with age. 
Arch. Biochem. Biophys. 397, 345-353 (2002).

121. Carter, E. L., Gupta, N. \& Ragsdale, S. W. High Affinity Heme Binding to a Heme Regulatory Motif on the Nuclear Receptor Rev-erb $\beta$ Leads to Its Degradation and Indirectly Regulates Its Interaction with Nuclear Receptor Corepressor. J. Biol. Chem. 291, 2196-2222 (2016).

122. Wickramasinghe, S., Porwit, A. \& Erber, W. Normal bone marrow cells: Development and cytology. Blood Bone Marrow Pathol. 19-44 (2011). doi:10.1016/B978-0-7020-3147-2.00002-X

123. Hartman, W. R. et al. CD38 expression, function, and gene resequencing in a human lymphoblastoid cell line-based model system. Leuk. Lymphoma $51,1315-25$ (2010).

124. Yu, Y.-R. A. et al. ORIGINAL RESEARCH Flow Cytometric Analysis of Myeloid Cells in Human Blood, Bronchoalveolar Lavage, and Lung Tissues. (2016). doi:10.1165/rcmb.2015-01460C

125. Matarraz, S. et al. Introduction to the diagnosis and classification of monocytic-lineage leukemias by flow cytometry. Cytom. Part B Clin. Cytom. 92, 218-227 (2017).

126. Stoneking, M. Hypervariable sites in the mtDNA control region are mutational hotspots. Am. J. Hum. Genet. 67, 1029-32 (2000).

127. Stewart, J. B. \& Chinnery, P. F. The dynamics of mitochondrial DNA heteroplasmy: implications for human health and disease. Nat. Rev. Genet. $16,530-542$ (2015).

128. Hall, A. CYTOPLASMIC BRIDGES BETWEEN ERYTHROBLASTS: $A$ LABORATORY CURIOSITY OR MEMBRANE NANOTUBULES WITH ESSENTIAL FUNCTIONS?(2016).

129. Savarino, A., Bottarel, F., Malavasi, F. \& Dianzani, U. Role of CD38 in HIV-1 infection: an epiphenomenon of T-cell activation or an active player in virus/host interactions? AIDS 14, (2000).

130. Nairz, M., Theurl, I., Swirski, F. K. \& Weiss, G. "Pumping iron"-how 
macrophages handle iron at the systemic, microenvironmental, and cellular levels. Pflügers Arch. - Eur. J. Physiol. 469, 397-418 (2017).

131. Wallace, D. C. \& Chalkia, D. Mitochondrial DNA genetics and the heteroplasmy conundrum in evolution and disease. Cold Spring Harb. Perspect. Biol. 5, a021220 (2013).

132. Ramos, A. et al. Frequency and Pattern of Heteroplasmy in the Complete Human Mitochondrial Genome. PLoS One 8, e74636 (2013).

133. Luo, S. et al. Biparental Inheritance of Mitochondrial DNA in Humans. Proc. Natl. Acad. Sci. U. S. A. 115, 13039-13044 (2018).

134. Mitchell, S. L. et al. Characterization of mitochondrial haplogroups in a large population-based sample from the United States. Hum. Genet. 133, 861-8 (2014).

135. Guo, W., Jiang, L., Bhasin, S., Khan, S. M. \& Swerdlow, R. H. DNA extraction procedures meaningfully influence qPCR-based mtDNA copy number determination. Mitochondrion 9, 261-265 (2009).

136. Moon, G. J. et al. Serum-mediated Activation of Bone Marrow-derived Mesenchymal Stem Cells in Ischemic Stroke Patients: A Novel Preconditioning Method. Cell Transplant. 27, 485-500 (2018). 
TE WHARE WANANGA OTE UPOKO OTEIKA A MAUI

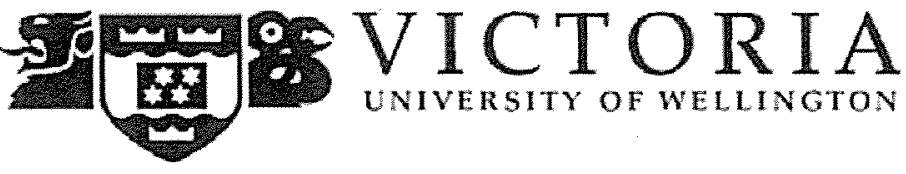

Availability and Deposit of Thesis Form - Open Research Archive

\begin{tabular}{|c|c|c|}
\hline Author's Name & \multicolumn{2}{|l|}{ Brittany Lewer } \\
\hline Thesis Title & \multicolumn{2}{|c|}{$\begin{array}{l}\text { Investigation of Mitodhondrial Transfer between } \\
\text { Human Erthroblasts. }\end{array}$} \\
\hline Degree Name & \multicolumn{2}{|c|}{ Master of Biomedical Science } \\
\hline Discipline & \multicolumn{2}{|c|}{ Biomedical Science. } \\
\hline School & \multicolumn{2}{|c|}{ School of Biolocical sciences } \\
\hline Supervisor(s) & \multicolumn{2}{|c|}{ Drmelanie meconnell Dr. Robert weinkove } \\
\hline Year & \multicolumn{2}{|c|}{2019} \\
\hline Keywords (3) & \multicolumn{2}{|c|}{ mitochondria, Witochondrial Transfer, Enthroblasts } \\
\hline Research Code & \multicolumn{2}{|c|}{0601} \\
\hline $\begin{array}{l}\text { Availability } \\
\text { Statement }\end{array}$ & \multicolumn{2}{|c|}{$\begin{array}{l}\text { I hereby consent to the above thesis being consulted, borrowed, copied or } \\
\text { reproduced in accordance with the provisions of the Library Regulations from } \\
\text { time to time made by the Academic Board. }\end{array}$} \\
\hline Signature & \multicolumn{2}{|c|}{ Bllevent } \\
\hline Date & \multicolumn{2}{|l|}{$12 / 9 / 19$} \\
\hline Deposit Declaration & \multicolumn{2}{|c|}{ 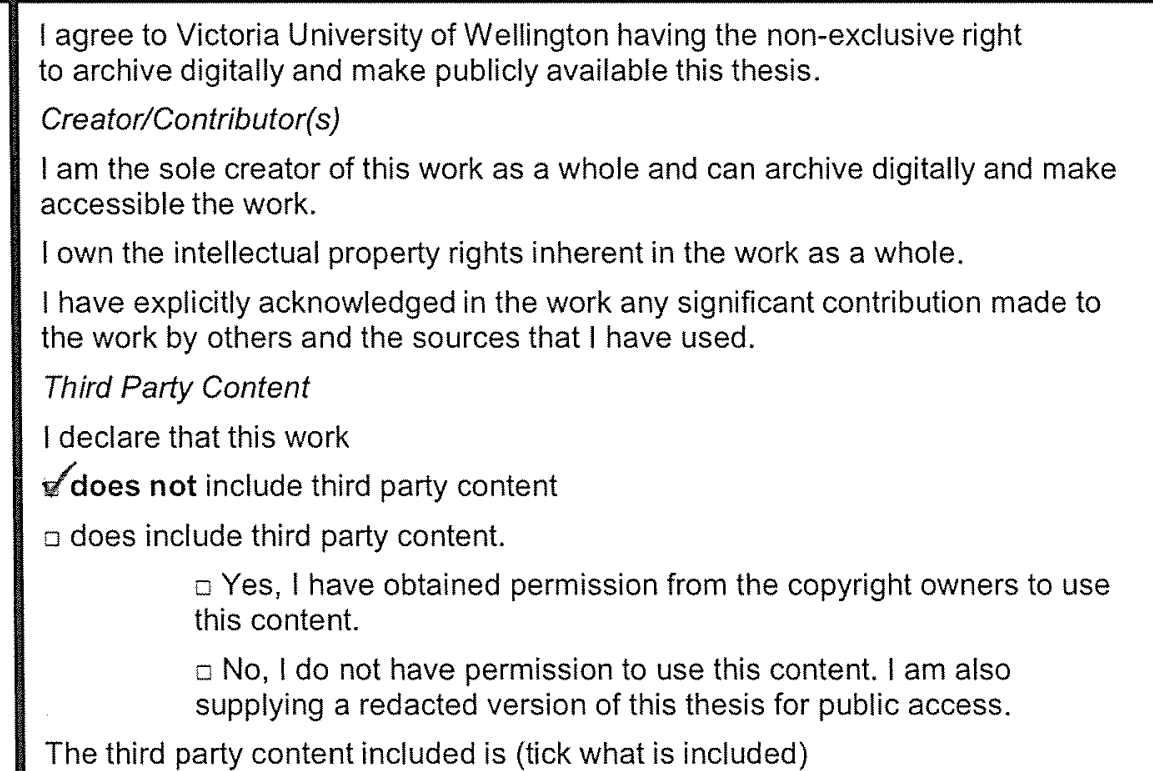 } \\
\hline Signature & blllow & \\
\hline Date & & \\
\hline
\end{tabular}

